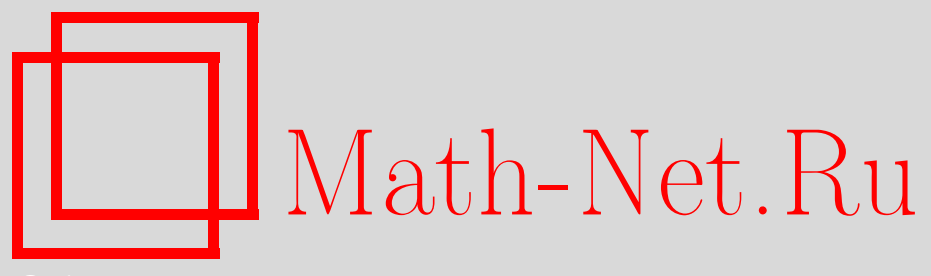

В. В. Шевчишин, Лагранжевы вложения бутылки Клейна и комбинаторные свойства группы классов отображений, Изв. РАН. Сер. матем., 2009, том 73, выпуск 4, 153-224

DOI: https://doi.org/10.4213/im2638

Использование Общероссийского математического портала Math-Net.Ru подразумевает, что вы прочитали и согласны с пользовательским соглашением http://www . mathnet.ru/rus/agreement

Параметры загрузки:

IP: 18.234 .156 .22

26 апреля 2023 г., 12:01:55

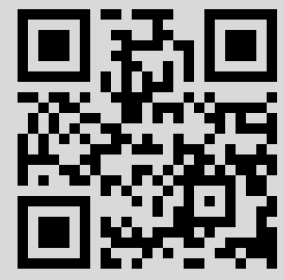




\title{
В. В. Шевчишин
}

\section{Лагранжевы вложения бутылки Клейна и комбинаторные свойства группы классов отображений}

\begin{abstract}
Доказано отсутствие лагранжевых вложений бутылки Клейна $K$ в $\mathbb{C P}^{2}$. Рассматриваются специальные вложения $K$ в симплектический пучок Лефшеца pr: $X \rightarrow S^{2}$ и изучается его монодромия. В качестве основного технического средства используется развитая в работе комбинаторная теория групп классов отображений. Показано, что если гомологический класс $[K] \in \mathrm{H}_{2}\left(X, \mathbb{Z}_{2}\right)$ тривиален, то монодромия семейства $\mathrm{pr}: X \rightarrow S^{2}$ должна иметь специальный вид. Оказывается, что такая монодромия не может быть реализована в случае $X=\mathbb{C P}^{2}$.
\end{abstract}

Библиография: 42 наименования.

Ключевые слова: симплектическая геометрия, лагранжевы подмногообразия, пучок Лефшеца, монодромия, группы классов отображений, системы Кокстера, группы Артина-Брискорна.

\section{Введение}

Целью настоящей работы является доказательство несуществования лагранжевых вложений бутылки Клейна $K:=\mathbb{R} \mathbb{P}^{2} \# \mathbb{R} \mathbb{P}^{2}$ в пространство $\mathbb{R}^{4}$ со стандартной симплектической структурой $\omega_{\mathrm{st}}:=d x_{1} \wedge d y_{1}+d x_{2} \wedge d y_{2}$. Это утверждение легко выводится из следующего результата.

ОСНОВНАЯ тЕОРЕМА. Если $K$ - вложенная лагранжева бутылка Клейна в компактном линейчатом симплектическом четырехмерном многообразии $(X, \omega)$, то ее класс $\mathbb{Z}_{2}$-гомологий $[K] \in \mathrm{H}_{2}\left(X, \mathbb{Z}_{2}\right)$ отличен от нуля.

Напомним, что симплектическое 4-многообразие $(X, \omega)$ называется линейчатым, если оно содержит вложенную симплектическую сферу $\Sigma \subset(X, \omega)$ с нулевым индексом самопересечения. Согласно [36] линейчатое симплектическое 4-многообразие $X$ симплектоморфно раздутию компактной комплексной линейчатой поверхности, причем $\omega$ соответствует кэлеровой форме.

Предположим, что существует вложенная лагранжева бутылка Клейна в $\left(\mathbb{R}^{4}, \omega_{\text {st }}\right)$. Тогда она лежит в некотором шаре. Растягивая и сдвигая этот шар, мы получаем вложенную лагранжеву бутылку Клейна в симплектическом шаре любого радиуса. По теореме Дарбу всякое симплектическое 4-многообразие $(X, \omega)$ содержит малый симплектический шар $B$ и, следовательно, вложенную лагранжеву бутылку Клейна $K$ в этом шаре $B$. Поскольку группа $\mathrm{H}_{2}\left(B, \mathbb{Z}_{2}\right)$ тривиальна, представленный бутылкой Клейна $K$ гомологический класс в многообразии $X$ тривиален. Применяя это построение к любому линейчатому

Работа выполнена при финансовой поддержке Гейзенберговской программы Немецкого исследовательского общества. 
4-многообразию $(X, \omega)$, мы получаем в нем вложенную лагранжеву бутылку Клейна с нулевым $\mathbb{Z}_{2}$-гомологическим классом $[K]$. Это противоречит основной теореме и тем самым доказывает несуществование лагранжевых вложений бутылки Клейна в $\left(\mathbb{R}^{4}, \omega_{\mathrm{st}}\right)$.

Основная теорема имеет и другие интересные приложения.

ТеОРема 0.1. Не существует лагранжева вложения бутылки Клейна $K$ в комплексную проективную плоскость $\mathbb{C P}^{2}$.

Выбор симплектической структуры на $\mathbb{C P}^{2}$ несуществен, поскольку по теореме Таубса любая симплектическая структура на $\mathbb{C P}^{2}$ симплектоморфна некоторому кратному формы Фубини-Штуди.

Для доказательства теоремы 0.1 заметим, что $\mathbb{Z}_{2}$-индекс самопересечения вложенной лагранжевой бутылки Клейна в любом симплектическом 4-многообразии $(X, \omega)$ равен ее $\mathbb{Z}_{2}$-классу Эйлера и, значит, обращается в нуль, в то время как $h^{2} \neq 0$ для единственного ненулевого класса $h \in \mathrm{H}_{2}\left(\mathbb{C P}^{2}, \mathbb{Z}_{2}\right)$. Таким образом, гомологический класс $[K] \in \mathrm{H}_{2}\left(\mathbb{C P}^{2}, \mathbb{Z}_{2}\right)$ лагранжево вложенной бутылки Клейна $K \subset \mathbb{C P}^{2}$ должен быть нулевым. С другой стороны, выполнив симплектическое раздутие $\mathbb{C P}^{2}$ в точке, не лежащей на $K$, мы получим поверхность Хирцебруха $\boldsymbol{F}_{1}$ с какой-то симплектической структурой, содержащую лагранжеву бутылку Клейна $K$ с нулевым классом гомологий $[K] \in \mathrm{H}_{2}\left(\boldsymbol{F}_{1}, \mathbb{Z}_{2}\right)$. Поскольку поверхности Хирцебруха линейчатые, это противоречит основной теореме.

Отметим, что лагранжевы вложения бутылки Клейна в раздутия $\mathbb{C P}^{2}$ существуют, как показывает следующий простой пример из [38]. Реализуем $K$ как вещественно-алгебраическое раздутие вещественной проективной плоскости $\mathbb{R P}^{2}$ и комплексифицируем это построение, получив раздутую в одной точке комплексную проективную плоскость $\mathbb{C P}^{2}$.

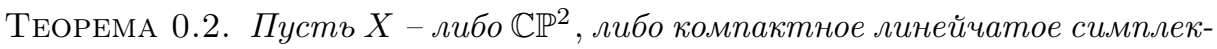
тическое 4-многообразие. Тогда образы любых двух трансверсальных лагранжевых вложений $\varphi_{1}, \varphi_{2}: \mathbb{R P}^{2} \rightarrow X$, представляющих один и тот же класс $\mathbb{Z}_{2}$-гомологий, имеют как минимум три точки пересечения.

В самом деле, $\mathbb{Z}_{2}$-индекс самопересечения для лагранжева вложения $\mathbb{R P}^{2} \rightarrow$ $X$ совпадает с приведенной по модулю 2 эйлеровой характеристикой $\mathbb{R P}^{2}$. Значит, образы вложений $\varphi_{1}$ и $\varphi_{2}$ имеют нечетное число точек пересечения. Если точка пересечения одна, то в ней можно сделать лагранжеву хирургию и получить лагранжево вложение бутылки Клейна $K$ (см. [4]). Класс $\mathbb{Z}_{2}$-гомологий этой бутылки Клейна равен сумме классов гомологий образов $\varphi_{1}$ и $\varphi_{2}$, т. е. тривиален, что противоречит основной теореме. Заметим, что в $\mathbb{C P}^{2}$ условие, налагаемое на класс гомологий, выполнено автоматически, поскольку в группе $\mathrm{H}_{2}\left(\mathbb{C P}^{2}, \mathbb{Z}_{2}\right)$ есть всего один ненулевой элемент.

Отметим также, что последний результат обобщает гипотезу Арнольда, поскольку мы не предполагаем, что вложения $\varphi_{1}\left(\mathbb{R P} \mathbb{P}^{2}\right), \varphi_{2}\left(\mathbb{R P}^{2}\right)$ изотопны (даже в классе гладких вложений).

Еще одно интересное приложение - характеризация гомологических классов, представимых лагранжевыми бутылками Клейна в $S^{2} \times S^{2}$. 
ТеОрема 0.3. Лагранжева бутылка Клейна $K$ в $S^{2} \times S^{2}$ с какой-либо симплектической структурой $\mathbb{Z}_{2}$-гомологична либо вертикальному, либо горизонталъному слою.

Во-первых, заметим, что всякая симплектическая структура на $S^{2} \times S^{2}$ симплектоморфна структуре вида $\omega=p_{1}^{*} \omega_{1}+p_{2}^{*} \omega_{2}$, где $p_{i}-$ проекции на сомножители (см. [36]). Группа $\mathrm{H}_{2}\left(S^{2} \times S^{2}, \mathbb{Z}_{2}\right)$ состоит из четырех элементов: нуля, $\mathbb{Z}_{2}$-классов гомологий слоев и $\mathbb{Z}_{2}$-класса гомологий диагонали $[\Delta]$. Поскольку $S^{2} \times S^{2}=\mathbb{C P}^{1} \times \mathbb{C P}^{1}$, очевидно, является линейчатым многообразием, класс $[K]$ не может быть нулевым по основной теореме.

Класс диагонали $[\Delta]$ исключается с помощью следующего рассуждения. М. Оден [4] показала, что эйлерова характеристика $\chi(V)$ вложенной лагранжевой неориентируемой поверхности $V$ в симплектическом четырехмерном многообразии $X$ удовлетворяет сравнению

$$
\chi(V) \equiv[V]^{2} \bmod 4,
$$

где $[V]^{2}$ - квадрат Понтрягина (двойственного по Пуанкаре) класса $[V] \in$ $\mathrm{H}_{2}\left(X, \mathbb{Z}_{2}\right)$. Значит, класс $[\Delta] \in \mathrm{H}_{2}\left(S^{2} \times S^{2}, \mathbb{Z}_{2}\right)$ не может быть реализован, так как $\chi(K)=0$, а квадрат Понтрягина диагонали $[\Delta]^{2}$ равен 2 .

Существование лагранжевых вложений бутылки Клейна, представляющих класс гомологий слоя проекции $p_{1}$, доказывается следующим образом. Выберем вложенную окружность $\Gamma$ в первом $S^{2}$-сомножителе. Для каждого $y \in \Gamma$ пусть $\boldsymbol{m}_{y}$ - меридиан в сфере $\{y\} \times S^{2}$, т. е. большая окружность, проходящая через северный и южный полюсы. Пусть окружности $\boldsymbol{m}_{y}$ гладко зависят от $y \in \Gamma$ и поворачиваются на угол $\pi$, когда точка $y$ делает полный оборот вдоль $\Gamma$. Тогда $K:=\bigcup_{y \in \Gamma} \boldsymbol{m}_{y}$ - неориентируемое $S^{1}$-расслоение над $\Gamma=S^{1}$, а значит, бутылка Клейна. По построению поверхность $K$ лагранжева относительно любой симплектической структуры вида $\omega=p_{1}^{*} \omega_{1}+p_{2}^{*} \omega_{2}$. Как будет показано в предложении 1.7, в этой ситуации $K$ гомологична слою проекции $p_{1}$.

Около 20 лет назад А. Б. Гивенталь [23] и М. Оден [4] показали, что любое лагранжево вложение замкнутой неориентируемой поверхности $V$ в симплектическое 4 -многообразие $(X, \omega)$ удовлетворяет сравнению (0.1). В частности, неориентируемая лагранжева поверхность $V$ в $\mathbb{R}^{4}$ должна удовлетворять сравнению $\chi(V) \equiv 0 \bmod 4$. А. Б. Гивенталь [23] построил явные примеры вложений всех допустимых поверхностей за исключением бутылки Клейна. М. Оден [4] предложила конструкцию лагранжева вложения бутылки Клейна $K$ в $\mathbb{C P}^{2}$, которая, как было замечено в [21], оказалась неверной, поскольку была основана на предположении о существовании двух лагранжевых вложений $\varphi_{1}, \varphi_{2}: \mathbb{R P}^{2} \rightarrow \mathbb{C P}^{2}$ с единственной точкой трансверсального пересечения. (Как отмечалось выше, такие вложения должны представлять единственный ненулевой класс в $h \in \mathrm{H}_{2}\left(\mathbb{C P}^{2}, \mathbb{Z}_{2}\right)$. Значит, сделанное М. Оден предположение противоречит теореме 0.2.) Отметим также, что условие (0.1) выполняется для более широкого класса неориентируемых вполне вещественных поверхностей $V$ в (почти) комплексных многообразиях, а пример вполне вещественной вложенной бутылки Клейна в $\mathbb{C}^{2}$ был построен В. Рудиным [41]. 
С. Ю. Немировский в работе [38] утверждал, что класс $\mathbb{Z}_{2}$-гомологий $[K]$ отличен от нуля для лагранжева вложения бутылки Клейна в любую комплексную алгебраическую поверхность, и использовал это утверждение для доказательства несуществования лагранжевых вложений бутылки Клейна в $\mathbb{C P}^{2}$. Однако в такой общности это утверждение неверно: мы строим к нему контрпример в п. 1.6. Еще одна попытка доказать теорему 0.1 была сделана К. Монке [37]. Он использовал совершенно другую технику, но и его доказательство, по-видимому, неполно.

0.1. Схема доказательства основной теоремы. С. Ю. Немировский показал в работе [38], что для заданного лагранжева подмногообразия $L$ в проективном многообразии $X$ и $S^{1}$-значной функции Морса $f: L \rightarrow S^{1}$ существуют изотопное $L$ подмногообразие $L^{\prime}$, раздутие $\widehat{X} \rightarrow X$ многообразия $X$ в комплексном подмногообразии $B \subset X, B \cap L^{\prime}=\varnothing$, и голоморфное семейство Лефшеца pr: $\widehat{X} \rightarrow \mathbb{C P}^{1}$ такие, что $\left(\widehat{X}, \mathrm{pr}, L^{\prime}\right)$ - семейство Морса-Лефшеца (см. определение 1.1). Д. Ору, В. Муньос и Ф. Пресас [7] обобщили это утверждение на случай произвольного компактного симплектического объемлющего многообразия $X$ (см. теорему 1.2). Нам понадобится небольшое уточнение их результата; оно доказывается в лемме 1.3 .

В случае $\operatorname{dim}_{\mathbb{R}} X=4$ группа гомологий $\mathrm{H}_{2}\left(X, \mathbb{Z}_{2}\right)$ естественным образом вкладывается в группу $\mathrm{H}_{2}\left(\widehat{X}, \mathbb{Z}_{2}\right)$, и потому раздутие не влияет на (не)тривиальность класса гомологий поверхности, не пересекающей раздуваемое множество. Поэтому мы можем считать, что семейство Лефшеца $\mathrm{pr}: X \rightarrow S^{2}$ задано сразу на $X$. В качестве функции Морса $f$ на $K$ возьмем (топологически единственное) расслоение $\operatorname{pr}_{K}: K \rightarrow S^{1}$ со слоем окружность. В результате мы получаем достаточно простую геометрическую конфигурацию, описанную ниже в лемме 1.3.

Основная идея в нашем подходе состоит в описании условий, необходимых для тривиальности класса гомологий $[K]$, в терминах семейства Лефшеца и его монодромии. Сначала мы находим выражение для $[K]$, исходя из гомологической спектральной последовательности. Это позволяет нам указать пример лагранжева вложения бутылки Клейна в проективную комплексную поверхность с нулевым классом гомологий $[K]$ (см. п. 1.6). Затем мы детально изучаем комбинаторную структуру группы классов отображений $\mathrm{Map}_{g}$. Развитая техника (см. п. 0.3) позволяет показать, что если класс $[K]$ тривиален, то монодромия построенного нами семейства Лефшеца должна иметь весьма специальный вид (см. предложение 3.1). Оказывается, что "подкручивая" подходящим образом часть такой специальной монодромии, можно получить новое симплектическое многообразие $X^{\prime}$, для которого

$$
\operatorname{rank} \mathrm{H}_{1}\left(X^{\prime}, \mathbb{Z}_{2}\right)=\operatorname{rank} \mathrm{H}_{1}\left(X, \mathbb{Z}_{2}\right)+1
$$

С другой стороны, используя классификацию линейчатых симплектических многообразий из работы [36], мы показываем, что в условиях основной теоремы многообразие $X^{\prime}$ также должно быть линейчатым, значит, ранги обеих групп $\mathrm{H}_{1}\left(X, \mathbb{Z}_{2}\right)$ и $\mathrm{H}_{1}\left(X^{\prime}, \mathbb{Z}_{2}\right)$ должны быть четными. Это противоречие завершает доказательство. 
0.2. Хирургия Люттингера и вполне вещественные вложения бутылки Клейна. После того как в мае 2006 г. появился препринт настоящей работы, С.Ю. Немировский нашел другое доказательство основной теоремы (см. [39]). Он заметил, что наша "подкрутка" многообразия $X$ есть хирургия Люттингера относительно бутылки Клейна $K$ (хорошо известная в случае (лагранжевых) вложений тора $T^{2}$; см. [6], [20], [33]). Он показал также, что равенство (0.2) может быть получено с помощью индекса Виро для кривых на бутылке Клейна $K$ и выполняется для любого вполне вещественного вложения $K$ в почти комплексное четырехмерное многообразие $(X, J)$.

0.3. Комбинаторная структура группы классов отображений. Как было сказано выше, доказательство основной теоремы основано на изучении монодромии семейств Лефшеца в группе классов отображений замкнутой поверхности. Наш ключевой технический результат в этом направлении - построение $\mathbb{Z}_{4}$-значных гомоморфизмов из некоторых "подфакторгрупп" $\widehat{\operatorname{Map}}_{g, \mu}$ (см. ниже) группы классов отображений Мap $_{g, 1}$ замкнутой поверхности $\Sigma$ рода $g \geqslant 2$ с одной отмеченной точкой $z_{0}$. Эти гомоморфизмы различают некоторые элементы в Map $_{g, 1}$ и, в том числе, элементы, возникающие в монодромии (топологических) семейств Лефшеца, содержащих специальным образом вложенные бутылки Клейна. А именно, определим группу $\widehat{\operatorname{Map}}_{g}$ с помощью расширения

$$
1 \rightarrow \mathrm{H}_{1}\left(\Sigma, \mathbb{Z}_{2}\right) \rightarrow{\widetilde{\operatorname{Map}_{g}}}^{\rightarrow \operatorname{Map}_{g}} \rightarrow 1
$$

так что $\widetilde{\operatorname{Map}}_{g}$ - это фактор группы $\operatorname{Map}_{g, 1}$ по образу ядра $\operatorname{Ker}\left(\pi_{1}\left(\Sigma, z_{0}\right) \rightarrow\right.$ $\left.\mathrm{H}_{1}\left(\Sigma, \mathbb{Z}_{2}\right)\right)$ при естественном вложении $\pi_{1}\left(\Sigma, z_{0}\right) \hookrightarrow \operatorname{Map}_{g, 1}$ (см. п. 1.3). Далее, пусть $\mu \in \mathrm{H}_{1}\left(\Sigma, \mathbb{Z}_{2}\right)$ - ненулевой элемент и $\widetilde{\text { Мар }}_{g, \mu}$ - стабилизатор элемента $\mu$ в $\widetilde{\operatorname{Map}}_{g}$.

ТЕОРема 0.4 (см. предложение 2.29). Существует гомоморфизм

$$
\widetilde{\varphi}_{\mu}: \widetilde{\operatorname{Map}}_{g, \mu} \rightarrow \mathbb{Z}_{4}
$$

со следующими свойствами:

( $\varphi 1) \widetilde{\varphi}_{\mu}\left(T_{\delta}\right) \equiv 0 \bmod 4$ для любого скручивания Дена $T_{\delta}, \delta \cap \mu \equiv 0 \bmod 2$;

$(\varphi 2) \widetilde{\varphi}_{\mu}\left(T_{\delta}^{2}\right) \equiv+1 \bmod 4$ для любого скручивания Дена, $[\delta] \cap[\mu] \equiv 1 \bmod 2$;

$(\varphi 3) \widetilde{\varphi}_{\mu}\left(\partial_{\pi}(\gamma)\right) \equiv(2 g-2) \varphi_{\mu}(\gamma) \bmod 4$ для любого элемента $\gamma \in \pi_{1}(\Sigma)$, где $\partial_{\pi}: \pi_{1}(\Sigma) \rightarrow \operatorname{Map}_{g, 1}-$ стандартное вложение;

(*) пусть $\alpha, \beta$ - простые замкнутые кривые на поверхности $\Sigma$, трансверсально пересекающиеся в единственной точке, причем $[\beta]_{\mathbb{Z}_{2}}=\mu \in \mathrm{H}_{1}\left(\Sigma, \mathbb{Z}_{2}\right)$; mогда $\widetilde{\varphi}_{\mu}\left(\left(T_{\alpha} T_{\beta}\right)^{3}\right) \equiv 2 \bmod 4$.

Отметим, что необращение в нуль значения $\widetilde{\varphi}_{\mu}\left(\left(T_{\alpha} T_{\beta}\right)^{3}\right) \equiv 2 \neq 0 \bmod 4$ в условии $(*)$ играет важную роль в доказательстве основной теоремы. Именно из этого свойства выводится соотношение (0.2), являющееся основным топологическим ингредиентом доказательства.

Для определения гомоморфизма $\varphi_{\mu}$ нам понадобятся эффективные методы вычислений в группе классов отображений $\operatorname{Map}_{g}$ поверхности $\Sigma$ рода $g \geqslant 1$. Это потребует понимания комбинаторной структуры группы $\mathrm{Map}_{g}$. 
М. Ден [17] заметил, что группа Мар ями, называемыми теперь скручиваниями Дена. При попытке найти простое копредставление для группы Мар отношением между скручиваниями Дена является соотношение группы кос. Поэтому естественно ожидать, что группа Map $_{g}$ должна напоминать группу кос, быть может, обобщенную. Действительно, копредставление Б. Вайнрыба [42] реализует группу Map $_{g}$ в качестве фактора некоторой группы кос Артина-Брискорна $\operatorname{Br}\left(\mathcal{S}_{g}\right)$ с простой системой образующих и соотношений. Недавно М. Мацумото [35] описал соотношения в копредставлении Вайнрыба в терминах так называемых элементов Гарсайда $\Delta(\mathcal{S})$, соответствующих некоторым подсистемам $\mathcal{S} \subset \mathcal{S}_{g}$. В $\S 2$ мы получаем ряд комбинаторных свойств копредставления Вайнрыба-Мацумото, которые существенно используются в доказательстве основной теоремы.

Напомним, что всякая группа кос Артина-Брискорна $\operatorname{Br}(\mathcal{S})$ строится по системе Кокстера $\left(\mathcal{S}, M_{\mathcal{S}}\right)$, где $\mathcal{S}$ - множество образующих группы $\operatorname{Br}(\mathcal{S})$ и $M_{\mathcal{S}}-$ матрица на $\mathcal{S}$, кодирующая комбинаторику соотношений. С каждой такой системой $\left(\mathcal{S}, M_{\mathcal{S}}\right)$ ассоциирована также так называемая группа Kокстера-Вейля $\mathrm{W}(\mathcal{S})$, получающаяся из группы $\operatorname{Br}(\mathcal{S})$ добавлением соотношения $s^{2}=1$ для каждой образующей $s \in \mathcal{S}$. В частности, имеется естественный сюръективный гомоморфизм $\operatorname{Br}(\mathcal{S}) \rightarrow \mathrm{W}(\mathcal{S})$, взаимно однозначный на множествах образующих. Ядро этого гомоморфизма называется группой крашеных кос, ассоциированной с системой $\mathcal{S}$, и обозначается $\mathrm{P}(\mathcal{S})$. Напомним, что квазиотражениями в группе $\mathrm{W}(\mathcal{S})$ и квазиобразующими в группе $\operatorname{Br}(\mathcal{S})$ называются элементы, сопряженные в этих группах стандартным образующим $s \in \mathcal{S}$. Обозначим через $\mathcal{T}(\mathcal{S})$ множество квазиотражений в группе $\mathrm{W}(\mathcal{S})$.

Tеорема 0.5 (см. теорему 2.11). Существуют естественные поднятия $\mathrm{W}(\mathcal{S}) \ni w \mapsto \widehat{w} \in \operatorname{Br}(\mathcal{S})$ u $\mathcal{T}(\mathcal{S}) \ni t \mapsto \tilde{t} \in \operatorname{Br}(\mathcal{S})$, для которых множество $\widetilde{\mathcal{T}}^{2}(\mathcal{S}):=\left\{\tilde{t}^{2}: t \in \mathcal{T}(\mathcal{S})\right\}$ является минималъным множеством образующих группы крашеных кос $\mathrm{P}(\mathcal{S})$.

Кроме того, абелианизачия $\mathrm{P}_{\mathrm{ab}}(\mathcal{S})$ группы крашеных кос $\mathrm{P}(\mathcal{S})$ является свободной абелевой группой с базисом $\left\{A_{t}: t \in \mathcal{T}(\mathcal{S})\right\}$, элементы которого проиндексированы множеством квазиотражений $\mathcal{T}(\mathcal{S})$, причем $A_{t}$ есть образ элемента $\tilde{t}^{2}$ при проекции в $\mathrm{P}_{\mathrm{ab}}(\mathcal{S})$.

Эти результаты хорошо известны для обычной группы кос $\mathrm{Br}_{d}$ и соответствующей группы крашеных кос $\mathrm{P}_{d}$. В частности, множество образующих в "стандартном" копредставлении группы $\mathrm{P}_{d}$ (см., например, [9]) есть в точности $\widetilde{\mathcal{T}}^{2}\left(\mathrm{~A}_{d-1}\right)$ для системы Кокстера $\mathrm{A}_{d-1}$, которая является системой Кокстера для группы $\mathrm{Br}_{d}$. Далее, поскольку соотношения в этом копредставлении могут быть выражены как произведения коммутаторов, абелианизация $\left(\mathrm{P}_{d}\right)_{\mathrm{ab}}$ является свободной абелевой группой с естественным базисом $\widetilde{\mathcal{T}}^{2}\left(\mathrm{~A}_{d-1}\right)$.

Подъемы в первой части теоремы 0.5 естественны в следующем смысле. В обоих случаях имеется естественное множество слов "простейшего типа," выражающих данный элемент $w \in \mathrm{W}(\mathcal{S})$ или, соответственно, $t \in \mathcal{T}(\mathcal{S})$, и мы доказываем, что значение этого слова в группе $\operatorname{Br}(\mathcal{S})$ зависит только от $w$ или, соответственно, $t$. Это означает, что имеются корректно определенные сопоставления $\mathrm{W}(\mathcal{S}) \ni w \mapsto \widehat{w} \in \operatorname{Br}(\mathcal{S})$ и $\mathcal{T}(\mathcal{S}) \ni t \mapsto \tilde{t} \in \operatorname{Br}(\mathcal{S})$. Простейшее слово 
для элемента $w \in \mathrm{W}(\mathcal{S})$ - это положительное слово минимально возможной длины. В случае квазиотражений $t \in \mathcal{T}(\mathcal{S})$ мы ищем слова, представляющие $t$ как элемент, сопряженный образующей, и имеющие простейший вид среди таких слов. Эти слова имеют вид $W=W^{\prime} \cdot s \cdot\left(W^{\prime}\right)^{-1}$, где $s \in \mathcal{S}$ и $W^{\prime}$ - положительное слово минимальной возможной длины. В частности, элементы $\tilde{t} \in \operatorname{Br}(\mathcal{S})$ являются квазиобразующими.

Если группа Кокстера-Вейля $\mathrm{W}(\mathcal{S})$ конечна, то она может быть реализована как группа, порожденная отражениями. Соответствующие системы Кокстера $\mathcal{S}$ полностью классифицированы. Для таких систем П. Делинь [18] и Е. Брискорн, K. Саито [13] определили так называемый элемент Гарсайда $\Delta(\mathcal{S}) \in \operatorname{Br}(\mathcal{S})$. В действительности элемент $\Delta(\mathcal{S})$ есть подъем $\widehat{w}_{\circ}$ (в смысле теоремы 0.5 ) единственного элемента наиболъшей длины $w_{\circ}$ в группе $\mathrm{W}(\mathcal{S})$. Квадрат $\Delta^{2}(\mathcal{S})$ лежит в центре группы $\mathrm{P}(\mathcal{S})$, и мы покажем в лемме 2.16 , что проекция элемента $\Delta^{2}(\mathcal{S})$ в группу $\mathrm{P}_{\mathrm{ab}}(\mathcal{S})$ есть в точности сумма $\sum_{t \in \mathcal{T}(\mathcal{S})} A_{t}$ всех базисных элементов в $\mathrm{P}_{\mathrm{ab}}(\mathcal{S})$. Отсюда немедленно находятся проекции соотношений Мацумото в группу $\mathrm{P}_{\mathrm{ab}}\left(\mathcal{S}_{g}\right)$.

Сформулируем теперь следующий важный результат.

Теорема 0.6 (см. теорему 2.13). Всякое разложение $\boldsymbol{t}=t_{1} \cdot t_{2} \cdots t_{l}$ единичного элемента $1 \in \mathrm{W}(\mathcal{S})$ в произведение квазиотражений $t_{i} \in \mathcal{T}(\mathcal{S})$ гурвиц-эквивалентно разложению в произведение квадратов квазиотражений, т.е. разложению вида $\boldsymbol{t}^{\prime}=t_{1}^{\prime} \cdot t_{2}^{\prime} \cdots t_{l}^{\prime}$, где $t_{2 i-1}^{\prime}=t_{2 i}^{\prime} \in \mathcal{T}(\mathcal{S})$.

Теорема 0.6 обобщает классический результат Гурвица. А именно, группа Кокстера-Вейля, соответствующая обычной группе кос $\mathrm{Br}_{d}$, есть симметрическая группа $\mathrm{Sym}_{d}$, и в этом случае утверждение теоремы эквивалентно неприводимости пространства разветвленных накрытий $f: C \rightarrow \mathbb{C P}^{1}$ заданной степени с фиксированным родом кривой $C$. В случае конечных групп Вейля, соответствующих простым комплексным алгебрам Ли, этот результат был доказан В. Каневым [31].

В свою очередь, теорема 0.6 используется для описания тех элементов в $\mathrm{P}(\mathcal{S})$, которые могут быть представлены в виде произведения квазиотражений $t_{i}$, проектирующихся в заданное подмножество $\mathcal{T}^{\prime} \subset \mathcal{T}(\mathcal{S})$. Очевидно, что вместо подмножества $\mathcal{T}^{\prime}$ можно рассматривать порожденную им подгруппу $G \subset \mathrm{W}(\mathcal{S})$. Для такой подгруппы $G$ мы обозначим через $\mathrm{P}_{\mathrm{ab}}(\mathcal{S})_{G}$ ее группу коинвариантов (см., например, [14]). Поскольку группа $\mathrm{W}(\mathcal{S})$ действует на $\mathrm{P}_{\mathrm{ab}}(\mathcal{S})$, переставляя элементы базиса $A_{t}, t \in \mathcal{T}(\mathcal{S})$, в абелевой группе $\mathrm{P}_{\mathrm{ab}}(\mathcal{S})_{G}$ имеется естественный базис, образованный множеством $G$-орбит в $\mathcal{T}(\mathcal{S})$. Мы будем обозначать элементы этого базиса через $A_{G \cdot t}$.

Теорема 0.7 (см. теорему 2.14). Пусть $G$ - подгруппа в $\mathrm{W}(\mathcal{S})$ и $x$ - элемент группь $\mathrm{P}(\mathcal{S})$, представимый в виде произведения: $x=\prod_{i} \hat{t}_{i}^{\epsilon_{i}} \cdot \prod_{j}\left[x_{2 j-1}, x_{2 j}\right]$, в котором $\hat{t}_{i}-$ квазиобразующие, $\epsilon_{i}= \pm 1, a\left[x_{2 j-1}, x_{2 j}\right]-$ коммутаторы элементов $x_{j} \in \operatorname{Br}(\mathcal{S})$. Предположим, что образы элементов $x_{i}$ и $\hat{t}_{j}$ в группе $\mathrm{W}(\mathcal{S})$ лежат в $G$. Тогда образ $[x]_{G}$ элемента $x$ в группе $\mathrm{P}_{\text {ар }}(\mathcal{S})_{G}$ лежит в свободной абелевой группе, порожденной базисными элементами $A_{G \cdot t}$ для $t \in G \cap \mathcal{T}(\mathcal{S})$. 
Смысл утверждения теоремы 0.7 состоит в том, что базисные элементы $A_{G \cdot t}$ при $t \notin G$ не появляются в разложении элемента $[x]_{G} \in \mathrm{P}_{\mathrm{ab}}(\mathcal{S})_{G}$.

Перейдем теперь к изучению структуры обобщенной группы кос $\operatorname{Br}\left(\mathcal{S}_{g}\right)$, свя-

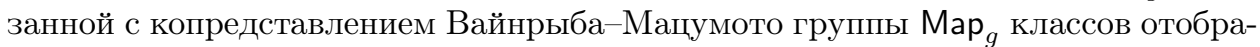
жений замкнутой поверхности $\Sigma$ рода $g$.

При $g \geqslant 4$ множество $\mathcal{T}\left(\mathcal{S}_{g}\right)$ бесконечно и не имеет внятного геометрического описания в терминах поверхности $\Sigma$. Мы показываем, что существует $\mathrm{W}\left(\mathcal{S}_{g}\right)$-эквивариантное отображение этого множества на множество ненулевых классов $v \in \mathrm{H}_{1}\left(\Sigma, \mathbb{Z}_{2}\right)$. Обозначая последнее множество через $\mathcal{H}_{g}$, мы получим $\mathrm{W}\left(\mathcal{S}_{g}\right)$-эквивариантный гомоморфизм свободных абелевых групп

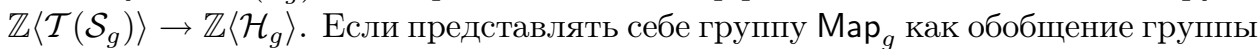
кос, то соответствующей ей группой Кокстера-Вейля будет группа $\boldsymbol{S p}\left(2 g, \mathbb{Z}_{2}\right)$, а квазиотражениями будут элементы множества $\mathcal{H}_{g}$. Отметим, что в случаях $g=1,2,3$ имеют место равенства $\mathcal{T}\left(\mathcal{S}_{g}\right)=\mathcal{H}_{g}$ и $\mathbf{W}\left(\mathcal{S}_{g}\right)=\boldsymbol{S} \boldsymbol{p}\left(2 g, \mathbb{Z}_{2}\right)$ (с точностью до изоморфного $\mathbb{Z}_{2}$ центра группы $\mathrm{W}\left(\mathcal{S}_{3}\right)$ в случае $g=3$ ).

Следующий результат описывает образующие ядра естественного гомоморфизма $\operatorname{Br}\left(\mathcal{S}_{g}\right) \rightarrow \boldsymbol{S p}\left(2 g, \mathbb{Z}_{2}\right)$. Это дает также образующие ядер гомоморфизмов $\operatorname{Map}_{g} \rightarrow \boldsymbol{S p}\left(2 g, \mathbb{Z}_{2}\right)$ и $\mathrm{W}\left(\mathcal{S}_{g}\right) \rightarrow \boldsymbol{S p}\left(2 g, \mathbb{Z}_{2}\right)$. Ядро второго из них мы будем называть группой Вейля-Торелли поверхности $\Sigma$ и обозначать $\mathrm{WI}_{g}$.

Теорема 0.8 (см. следствие 2.23, предложение 2.26). Справедливы следующие утверждения:

i) ядро гомоморфизма $\operatorname{Map}_{g} \rightarrow \boldsymbol{S p}\left(2 g, \mathbb{Z}_{2}\right)$ порождается квадратами $T_{\delta}^{2}$ скручиваний Дена относительно неразбивающих кривых $\delta \subset \Sigma$;

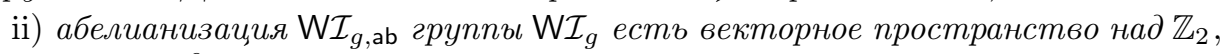
изоморфное $\wedge{ }^{6} \mathrm{H}_{1}\left(\Sigma, \mathbb{Z}_{2}\right)$;

iii) существует естественное расширение

$$
0 \rightarrow \mathbb{Z}\left\langle\mathcal{H}_{g}\right\rangle \rightarrow \Lambda_{g} \rightarrow \mathrm{W} \mathcal{I}_{g, \text { ab }} \rightarrow 0
$$

в котором группа $\Lambda_{g}$ может быть реализована как подрешетка в $\frac{1}{2} \mathbb{Z}\left\langle\mathcal{H}_{g}\right\rangle$, а образ индуцированного вложения $\mathrm{W} \mathcal{I}_{g, \text { ab }} \subset \frac{1}{2} \mathbb{Z}\left\langle\mathcal{H}_{g}\right\rangle / \mathbb{Z}\left\langle\mathcal{H}_{g}\right\rangle \cong \mathbb{Z}_{2}\left\langle\mathcal{H}_{g}\right\rangle$ порождается суммами вида $L_{V}:=\sum_{v \neq 0 \in V} A_{v}$, где $V$ - произвольное симплектическое шестимерное $\mathbb{Z}_{2}$-векторное подпространство в $\mathrm{H}_{1}\left(\Sigma, \mathbb{Z}_{2}\right)$;

iv) существуют расширение групп

$$
0 \rightarrow \Lambda_{g} \rightarrow \widehat{\boldsymbol{S p}}\left(2 g, \mathbb{Z}_{2}\right) \rightarrow \boldsymbol{S p}\left(2 g, \mathbb{Z}_{2}\right) \rightarrow 1
$$

и гомоморфизм из расширения $1 \rightarrow \mathrm{P}\left(\mathcal{S}_{g}\right) \rightarrow \operatorname{Br}\left(\mathcal{S}_{g}\right) \rightarrow \mathrm{W}\left(\mathcal{S}_{g}\right) \rightarrow 1$ на это расширение, для которого гомоморфизм $\mathrm{W}\left(\mathcal{S}_{g}\right) \rightarrow \boldsymbol{S p}\left(2 g, \mathbb{Z}_{2}\right)$ имеет обычныи смисл, а гомоморфизм $\mathrm{P}\left(\mathcal{S}_{g}\right) \rightarrow \Lambda_{g}$ есть композиция $\mathrm{P}\left(\mathcal{S}_{g}\right) \rightarrow \mathrm{P}_{\mathrm{ab}}\left(\mathcal{S}_{g}\right) \rightarrow$ $\mathbb{Z}\left\langle\mathcal{H}_{g}\right\rangle \subset \Lambda_{g}$

В результате мы можем выполнять все вычисления в существенно меньших и геометрически более понятных группах $\Lambda_{g}$ и $\widehat{\boldsymbol{S p}}\left(2 g, \mathbb{Z}_{2}\right)$, а не в группе $\mathrm{Map}_{g}$ или $\operatorname{Br}\left(\mathcal{S}_{g}\right)$. По существу это означает, что мы можем работать в группе $\Lambda_{g}$, рассматриваемой как $\boldsymbol{S p}\left(2 g, \mathbb{Z}_{2}\right)$-модуль.

Для любого класса $\mu \in \mathrm{H}_{1}\left(\Sigma, \mathbb{Z}_{2}\right)$ мы обозначаем через $\varphi_{\mu}: \mathrm{H}_{1}\left(\Sigma, \mathbb{Z}_{2}\right) \rightarrow \mathbb{Z}_{2}$ гомоморфизм, задаваемый формулой $v \mapsto v \cap \mu$, и определяем гомоморфизм 
$\widehat{\varphi}_{\mu}: \mathbb{Z}\left\langle\mathcal{H}_{g}\right\rangle \rightarrow \mathbb{Z}$, положив $\widehat{\varphi}_{\mu}\left(A_{v}\right):=1 \in \mathbb{Z}$, если $\varphi_{\mu}(v) \equiv 1 \in \mathbb{Z}_{2}$, и $\widehat{\varphi}_{\mu}\left(A_{v}\right):=0$ в противном случае. Таким образом, $\widehat{\varphi}_{\mu}$ есть "считающая функция" для отображения множеств $\varphi_{\mu}: \mathcal{H}_{g} \rightarrow \mathbb{Z}_{2}$. Наш результат касается группы классов отображений $\operatorname{Map}_{g, 1}$ поверхности $\Sigma$ с одной отмеченной точкой. Известно, что при любом $g \geqslant 2$ ядро естественного гомоморфизма $\mathrm{Map}_{g, 1} \rightarrow$ Map $_{g}$ изоморфно фундаментальной группе $\pi_{1}(\Sigma)$.

ТЕОРема 0.9 (см. следствие 2.28). Гомоморфизм $\widehat{\varphi}_{\mu}: \mathbb{Z}\left\langle\mathcal{H}_{g}\right\rangle \rightarrow \mathbb{Z}$ eстественным образом продолжается на стабилизатор $\operatorname{Br}\left(\mathcal{S}_{g}\right)_{\mu}$ элемента $\mu$. Это продолжение индуцирует гомоморфизм $\widehat{\varphi}_{\mu}: \operatorname{Map}_{g, 1, \mu} \rightarrow \mathbb{Z}_{4}$. Кроме того, $\widehat{\varphi}_{\mu}\left(x_{\gamma}\right) \equiv(2 g-2) \varphi_{\mu}(\gamma) \bmod 4$ для любого элемента $\gamma \in \pi_{1}(\Sigma)$ и соответствующего ему элемента $x_{\gamma} \in \operatorname{Map}_{g, 1}$.

Этот результат используется при доказательстве ключевой теоремы 0.4. В качестве еще одного следствия мы получаем следующий результат.

Теорема 0.10. Любое топологическое семейство Лефиеца $\mathrm{pr}: X \rightarrow Y$ со слоем $F:=\operatorname{pr}^{-1}(y)$ четного рода $g=2 g^{\prime}$ имеет $\mathbb{Z}_{2}$-сечение, т.е. такой класс $[\sigma] \in \mathrm{H}_{2}\left(X, \mathbb{Z}_{2}\right)$, ито $[\sigma] \cap[F] \equiv 1 \bmod 2$.

Теорема 0.10 доказана в теореме 2.30 в форме задачи о разложении на множители в группе Map $_{g, 1}$.

Кроме того, в следствии 2.25 доказывается следующее утверждение.

ТЕОРема 0.11. При $g \geqslant 2$ существуют вложение

$$
\mathrm{H}_{1}\left(\Sigma, \mathbb{Z}_{2}\right) \hookrightarrow \mathrm{T}^{3}\left(\mathrm{H}_{1}\left(\Sigma, \mathbb{Z}_{2}\right)\right)=: \mathrm{T}^{3}
$$

в третью тензорную степень и гомоморфизм ю из $\operatorname{Ker}\left(\operatorname{Map}_{g, 1} \rightarrow \boldsymbol{S p}\left(2 g, \mathbb{Z}_{2}\right)\right)$ в $\mathrm{T}^{3}$ такие, что композииия гомоморфизма ю с вложением $\pi_{1}(\Sigma) \hookrightarrow \operatorname{Map}_{g, 1}$ равна композиции проекции $\pi_{1}(\Sigma) \rightarrow \mathrm{H}_{1}\left(\Sigma, \mathbb{Z}_{2}\right)$ с вложением $\mathrm{H}_{1}\left(\Sigma, \mathbb{Z}_{2}\right) \hookrightarrow \mathrm{T}^{3}$.

Объясним теперь, как теорема 0.7 используется в доказательстве основного результата. Напомним, что согласно [22, теорема C] группа Мap $g$ при $g \geqslant 3$ имеет универсальное центральное расширение $\widetilde{\operatorname{Map}}_{g}$ с ядром $\mathbb{Z}$. Существует естественный подъем монодромии топологического семейства Лефшеца $\mathrm{pr}: X \rightarrow Y$ в расширение $\widetilde{\operatorname{Map}_{g}}$, при котором полная монодромия есть элемент $c$ в ядре, т. е. целое число. Расширение Жерве можно определить как прообраз универсального накрытия

$$
0 \rightarrow \mathbb{Z} \rightarrow \widetilde{\boldsymbol{S p}}(2 g, \mathbb{R}) \rightarrow \boldsymbol{S p}(2 g, \mathbb{R}) \rightarrow 0
$$

и можно показать, что число $c$ есть, по существу, число Черна $c_{1}$ пучка симплектических векторных пространств $R^{1} \mathrm{pr}_{*} \underline{\mathbb{R}}_{X}$ - первого прямого образа постоянного пучка $\mathbb{R}_{X}$ над $X$ (ср. с [1], [11], [12]). Напомним, что слой пучка $R^{1} \operatorname{pr}_{*} \underline{\mathbb{R}}_{X}$ над регулярным значением $y \in Y$ есть просто группа когомологий $\mathrm{H}^{1}\left(X_{y}, \mathbb{R}\right)$ слоя $X_{y}:=\operatorname{pr}^{-1}(y) \cong \Sigma_{g}$, а симплектическая структура на $\mathrm{H}^{1}\left(X_{y}, \mathbb{R}\right)$ задается $\cup$-умножением. Данное С. Жерве описание расширения $\widetilde{\operatorname{Map}}_{g}$ показывает, что образующая ядра $\mathbb{Z}$ есть цепной элемент (см. п. 2.2), так что $c_{1}$ 
определяет, сколько раз с учетом знака цепное соотношение встречается в полной монодромии. Более того, универсальность расширения $\widehat{\operatorname{Map}}_{g}$ означает, что никаких дальнейших инвариантов найти нельзя.

Пусть теперь $G$ - подгруппа в Мар $_{g}$. Тогда может существовать нетривиальное центральное расширение $0 \rightarrow A \rightarrow \widehat{G} \rightarrow G \rightarrow 0$ для “большей” абелевой группы $A$, не сводящееся к расширению $\widehat{\operatorname{Map}_{g}}$. В этом случае подходящие гомоморфизмы $\psi: A \rightarrow \mathbb{Z}$ или $\psi: A \rightarrow \mathbb{Z}_{m}$ будут задавать (новые) "характеристические классы" для семейств Лефшеца с монодромией в группе $G$. Теорема 0.7 объясняет, как можно построить такое расширение $0 \rightarrow A \rightarrow \widehat{G} \rightarrow G \rightarrow 0$ в случае, когда группа $G$ есть прообраз некоторой подгруппы $H<\boldsymbol{S p}\left(2 g, \mathbb{Z}_{2}\right)$ при естественном гомоморфизме $\operatorname{Map}_{g} \rightarrow \boldsymbol{S p}\left(2 g, \mathbb{Z}_{2}\right)$. При этом гомоморфизм $\widehat{\varphi}_{\mu}$ из теоремы 0.9 является, по существу, примером гомоморфизма $\psi$.

0.4. Обозначения. Пусть $X$ - четырехмерное многообразие, а $Y$ - ориентированная поверхность. Для отображения $\mathrm{pr}: X \rightarrow Y$ и подмножества $U \subset Y$ мы обозначаем $X_{U}:=\operatorname{pr}^{-1}(U)$. Например, $X_{y}=\operatorname{pr}^{-1}(y)$ - это слой над точкой $y \in Y$.

Пусть $\Sigma$ - это $C W$-комплекс и $f: \Sigma \rightarrow \Sigma$ - непрерывное отображение. Тором отображения $f$ называется факторпространство $Z$ цилиндра $\Sigma \times[0,1]$ по отношению эквивалентности $(z, 1) \sim(f(z), 0)$. В дальнейшем $\Sigma$ будет вещественной поверхностью, а отображение $f: \Sigma \rightarrow \Sigma$ - диффеоморфизмом. Заметим, что отображение pr: $[z, t] \in(\Sigma \times[0,1] / \sim) \mapsto t \in S^{1}=[0,1] /(0 \sim 1)$ определяет проекцию pr: $Z \rightarrow S^{1}$ расслоения с тотальным пространством $Z$ и слоем $\Sigma$. Мы будем рассматривать это расслоение как часть структуры тора на $Z$. Обратно, для всякого расслоения $\mathrm{pr}: Z \rightarrow S^{1}$ со слоем $\Sigma$ существует непрерывное отображение $f: \Sigma \rightarrow \Sigma$, для которого $(Z, \mathrm{pr})$ является тором. В этом случае отображение $f: \Sigma \rightarrow \Sigma$ называется отображением монодромии расслоения $\mathrm{pr}: Z \rightarrow S^{1}$, или просто монодромией $\mathrm{pr}: Z \rightarrow S^{1}$. Монодромия определена с точностью до изотопии, и два расслоения $\mathrm{pr}: Z \rightarrow S^{1}$ и $_{\mathrm{pr}}^{\prime}: Z^{\prime} \rightarrow S^{1}$ изоморфны тогда и только тогда, когда их монодромии изотопны. Более общим образом, пусть pr: $Z \rightarrow Y$ - расслоение со слоем $\Sigma$ и $\gamma: S^{1} \rightarrow Y$ - петля в его базе. Тогда монодромия индуцированного расслоения $\gamma^{*} Z:=Z \times_{Y, \gamma} S^{1}$ называется монодромией расслоения $\mathrm{pr}: Z \rightarrow Y$ вдоль кривой $\gamma$. Монодромия определяет гомоморфизм монодромии из фундаментальной группы $\pi_{1}\left(Y, y_{0}\right)$ в группу классов отображений слоя $\Sigma$.

Пусть pr: $Z \rightarrow S^{1}$ - расслоение со слоем $\Sigma, y_{0} \in S^{1}$ - отмеченная точка в его базе, а $\Gamma \subset Z$ - сечение расслоения $\mathrm{pr}$ (т. е. $\mathrm{pr}: \Gamma \rightarrow S^{1}$ - гомеоморфизм). Положим $\Sigma=\operatorname{pr}^{-1}\left(y_{0}\right)$ и $z_{0}:=\Gamma \cap \Sigma$. Тогда $(Z, \mathrm{pr})$ может быть реализовано как тор отображения $f \in \mathscr{D}$ iff $\left(\Sigma, z_{0}\right)$, для которого $\Gamma \subset Z$ является тором отображения $f:\left\{z_{0}\right\} \rightarrow\left\{z_{0}\right\}$. Такое отображение $f$ единственно с точностью до изотопии в группе $\mathscr{D}$ iff $\left(\Sigma, z_{0}\right)$, и мы называем $f \in \mathscr{D}$ iff $\left(\Sigma, z_{0}\right)$ монодромией $\operatorname{pr}: Z \rightarrow S^{1}$, соответствующей сечению $\Gamma$. Аналогичная терминология используется и в случае произвольного расслоения $\mathrm{pr}: Z \rightarrow Y$ со слоем $\Sigma$. Поверхность $\Sigma$ всегда будет ориентированной, а отображение $f$ - сохраняющим ориентацию (т. е. $\left.f \in \mathscr{D} i f f_{+}\left(\Sigma, z_{0}\right)\right)$. 
Вложенная окружность $\delta$ на связной поверхности $\Sigma$ называется несущественной, если она ограничивает диск в $\Sigma$, и существенной в противном случае. Все окружности, встречающиеся в доказательствах, будут существенными. Вложенная окружность $\delta$ на поверхности $\Sigma$ называется разбивающей, если $\Sigma \backslash \delta$ имеет две компоненты связности, и неразбивающей в противном случае. Если поверхность $\Sigma$ замкнута и ориентируема, то окружность $\delta$ является неразбивающей тогда и только тогда, когда $[\delta] \neq 0 \in \mathrm{H}_{1}(\Sigma, \mathbb{Z})$, или, эквивалентным образом, $[\delta] \neq 0 \in \mathrm{H}_{1}\left(\Sigma, \mathbb{Z}_{2}\right)$.

Автор признателен С. Ю. Немировскому за многочисленные обсуждения. Д. Н. Ахиезер, Д. Ору, К. Цилибак, Я. М. Элиашберг, У. Хаменштэдт, С. М. Ивашкович, В. М. Харламов, Д. Котшик, С. Ю. Оревков, Л. В. Полтерович и В. Зёргель сделали много полезных замечаний к предыдущим вариантам работы.

\section{§1. Топология семейств Лефшеца}

1.1. Лагранжевы подмногообразия в семействах Лефшеца. Определения топологического семейства Лефшеца $\mathrm{pr}: X \rightarrow Y$, топологического пучка Лефшеца $\mathrm{pr}: X \rightarrow S^{2}$ и (симплектического) раздутия такого $X$ можно найти в книге [24], а общая теория симплектических семейств Лефшеца и лагранжевых подмногообразий в них развивается в работах [7] и [19]. В статье [38] обсуждается случай лагранжевых подмногообразий в проективных алгебраических многообразиях. В настоящей работе рассматривается только случай, когда объемлющее многообразие четырехмерно, а база семейства Лефшеца является ориентированной поверхностью.

ОПРЕДЕЛЕНИЕ 1.1. Пусть $X$ - замкнутое четырехмерное многообразие, а $L \subset X$ - замкнутая вложенная поверхность. Семейство Морса-Лефшеща на $(X, L)$ - это семейство Лефшеца $\mathrm{pr}: X \rightarrow Y$ со следующими свойствами:

i) образ $\operatorname{pr}(L)$ есть гладкая вложенная кривая $\Gamma$ в $Y$;

ii) ограничение проекции $\operatorname{pr}_{L}:=\left.\operatorname{pr}\right|_{L}: L \rightarrow \Gamma$ имеет только морсовские особые точки;

iii) $x \in L$ является критической точкой для $\mathrm{pr}_{L}: L \rightarrow \Gamma$ тогда и только тогда, когда она является критической точкой для $\mathrm{pr}: X \rightarrow Y$.

Кривая Г должна быть окружностью или дугой окружности. Оказывается, что для любого симплектического многообразия $(X, \omega)$ и вложенной в него лагранжевой замкнутой поверхности $L \subset X$ существует симплектическое семейство Морса-Лефшеца над двумерной сферой $S^{2}$. А именно, следующий результат был доказан в работе [7].

Теорема 1.2. Пусть $(X, \omega)$ - замкнутое симплектическое четырехмерное многообразие такое, что $[\omega]=c_{1}(\mathscr{L})$ для некоторого линейного расслоения $\mathscr{L}$ на $X$, и пусть $L \subset X$ - замкнутое лагранжево подмногообразие, a $f: L \rightarrow S^{1}$ - функиия Морса. Тогда существуют симплектическое раздутие $\widehat{X} \rightarrow X$ в конечном множестве точек $B=\left\{b_{1}, \ldots, b_{N}\right\} \subset X \backslash L$, семейство морса-Лефиеца $\mathrm{pr}: \widehat{X} \rightarrow S^{2}$ для $(X, \omega, L)$ и вложение $\gamma: S^{1} \hookrightarrow S^{2}$, для котоpux $\left.\operatorname{pr}\right|_{L}=\gamma \circ f$. 
Заметим, что в случае обычной функции Морса $f: L \rightarrow \mathbb{R}$ множество ее значений $f(L)$ является отрезком, который может быть вложен в окружность $S^{1}$. Поэтому этот случай сводится к случаю функций Морса со значениями в окружности.

Установим теперь вариант теоремы 1.2, необходимый для наших целей.

Лемма 1.3. Для любого лагранжева вложения бутылки Клейна $K$ в симплектическое четырехмерное многообразие $(X, \omega)$ существуют деформация $\widetilde{\omega}$ симплектической формы $\omega$, симплектическое раздутие $\widehat{X}$ многообразия $X$ в конечном числе точек $x_{1}, \ldots, x_{N} \in X$ вне $K$ и симплектическое семейство Лефшеца pr: $\widehat{X} \rightarrow S^{2}$ со следующими свойствами:

(ML1) образ $\operatorname{pr}(K)$ есть вложенная окружность $\Gamma \subset S^{2}$;

(ML2) никакое критическое значение $y \in S^{2}$ проекции $\operatorname{pr}: X \rightarrow S^{2}$ не лежит на Г;

(ML3) все слои ограничения проекции $\left.\mathrm{pr}\right|_{K}: K \rightarrow \Gamma$ являются окружностями;

(ML4) существует сечение проекции $\mathrm{pr}: X \rightarrow S^{2}$ (m.е. поверхность $S \subset X$, проекиия которой $\left.\mathrm{pr}\right|_{S}: S \rightarrow S^{2}$ на базу есть диффеоморфизм), для которого $S \cap K=\varnothing$.

ДокАЗАтЕльство. Деформация симплектической формы $\omega$ необходима для того, чтобы сделать класс когомологий $[\omega]$ рациональным. Легко показать, что для всякой римановой метрики $g_{X}$ на многообразии $X$ и любого $\varepsilon>0$ существует замкнутая 2-форма $\widetilde{\omega}$ такая, что класс $[\widetilde{\omega}]$ рационален и $\|\widetilde{\omega}-\omega\|_{C^{0}(X)} \leqslant \varepsilon$. Последнее условие гарантирует, что $\widetilde{\omega}$ также является симплектической формой.

Поскольку $\mathrm{H}^{2}(K, \mathbb{R})=0$, ограничение $\left.\widetilde{\omega}\right|_{K}$ будет точной формой. Кроме того, из теории Ходжа следует, что найдется 1-форма $\alpha$ на $K$, для которой $d \alpha=\left.\widetilde{\omega}\right|_{K}$ и

$$
\|\alpha\|_{C^{0}(K)} \leqslant C\left\|\left.\widetilde{\omega}\right|_{K}\right\|_{C^{0}(K)}=C\left\|\left.(\widetilde{\omega}-\omega)\right|_{K}\right\|_{C^{0}(K)} \leqslant C \varepsilon
$$

где константа $C$ зависит только от вложения $K \subset X$ и метрики $g_{X}$. В силу (обобщенной) теоремы Дарбу (см., например, [2]) найдется окрестность $U$ бутылки Клейна $K$ в многообразии $X$, которая симплектоморфна окрестности $U^{\prime}$ нулевого сечения кокасательного расслоения $T^{*} K$ с канонической симплектической формой $\omega_{T^{*} K}$, причем $K$ переходит при этом симплектоморфизме в нулевое сечение. Условие $\|\alpha\|_{C^{0}(K)} \leqslant C \varepsilon$ гарантирует, что график $L_{\alpha} \subset T^{*} K$ формы $\alpha$ содержится в $U^{\prime}$. Заметим, что $\left.\omega_{T^{*} K}\right|_{L_{\alpha}}=d \alpha=\widetilde{\omega}$. Следовательно, существует диффеоморфизм $\Phi: U \rightarrow U$, тождественный вблизи границы $\partial U$ и такой, что $\left.\Phi^{*} \widetilde{\omega}\right|_{K}=\left.\omega\right|_{K}=0$. Если теперь обозначить через $\widetilde{\omega}$ форму $\Phi^{*} \widetilde{\omega}$, то $K$ будет лагранжева относительно этой формы с рациональным классом когомологий.

Теперь требуемое симплектическое семейство Лефшеца получается применением теоремы 1.2 к отображению $\varphi: K \rightarrow S^{1}$, реализующему бутылку Клейна $K$ как нетривиальное $S^{1}$-расслоение над окружностью $S^{1}$. Общее число раздутий в $\widehat{X} \rightarrow X$ должно быть больши́м по следующей причине. По построению симплектического семейства Лефшеца $\mathrm{pr}: \widehat{X} \rightarrow S^{2}$ в работе [7] центры этих 
раздутий $x_{1}, \ldots, x_{N}$ есть в точности общие нули пары сечений $s_{0}, s_{1}$ комплексного линейного расслоения $\mathscr{L}$ на многообразии $X$, для которого $c_{1}(\mathscr{L})=k[\widetilde{\omega}]$ при некотором $k \gg 0$. Кроме того, индекс пересечения поверхностей $s_{0}^{-1}(0)$ и $s_{1}^{-1}(0)$ равен +1 в каждой точке $x_{1}, \ldots, x_{N}$, так что $N=k^{2}[\widetilde{\omega}]^{2} \gg 0$. Лемма доказана.

1.2. Топология семейства Лефшеца вблизи особого слоя. Пусть $\Sigma$ ориентированная поверхность, а $\delta \subset \Sigma$ - вложенная окружность. Зафиксируем кольцеобразную окрестность $U$ окружности $\delta$ и отождествим ее с кольцом $\left\{(\rho, \theta): \frac{1}{2}<\rho<2,0 \leqslant \theta \leqslant 2 \pi\right\}$, на котором ориентация задана формой $d \rho \wedge d \theta$. Пусть $\chi=\chi(\rho)$ - неубывающая функция такая, что $\chi(\rho) \equiv 0$ при $\rho \leqslant \frac{1}{2}$ и $\chi(\rho) \equiv 2 \pi$ при $\rho \geqslant 2$.

ОПРЕДЕЛЕНИЕ 1.4. Положителъным скручиванием Дена поверхности $\Sigma$ относительно кривой $\delta$ называется диффеоморфизм $T_{\delta}: \Sigma \rightarrow \Sigma$, тождественный вне окрестности $U$ и задаваемый формулой

$$
T_{\delta}(\rho, \theta)=(\rho, \theta-\chi(\rho))
$$

внутри $U$. Отрицательным скручиванием Дена называется обратный диффеоморфизм; он задается формулой $(\rho, \theta) \mapsto(\rho, \theta+\chi(\rho))$.

Скручивание Дена заданного знака единственно с точностью до изотопии. Знак выбирается так, чтобы в монодромии симплектических (и обычных алгебраических) семейств Лефшеца встречались только положительные скручивания Дена.

Положительность скручиваний Дена в симплектических семействах Лефшеца не будет использоваться в доказательстве наших результатов о группе классов отображений. Поэтому они применимы к любым топологическим семействам Лефшеца. Примером такого приложения является сформулированная во введении теорема 0.10.

Обозначение $T_{\delta}$ будет использоваться как для скручивания Дена относительно конкретной вложенной окружности $\delta \subset \Sigma$ с заданной окрестностью $U$ с координатами $(\rho, \theta)$, так и для класса изотопии этого скручивания в соответствующей группе классов отображений (см. ниже п. 1.3).

Пусть $\Delta$ - диск с границей $\partial \Delta=: \gamma, Z$ - четырехмерное многообразие, а pr: $Z \rightarrow \Delta-$ собственное семейство Лефшеца с единственной критической точкой $z^{*}$, лежащей над началом координат $0 \in \Delta$. Обозначим через $\Sigma$ общий слой проекции $\mathrm{pr}: Z \rightarrow \Delta$, скажем, над точкой $y_{0} \in \gamma$.

ЛЕмма 1.5. 1) Особый слой $\Sigma^{*}:=\operatorname{pr}^{-1}(0)$ является дебормационным ретрактом многообразия $Z$.

2) Монодромия вдоль граничной окружности д $\Delta$ действует на слое $\Sigma$ как скручивание Дена относительно некоторой вложенной окружности $\delta \subset \Sigma, u$ особый слой $\Sigma^{*}$ получается из $\Sigma$ стягиванием этой окружности $\delta$ в двойную точку на особом слое $\Sigma^{*}$.

3) Если окружность $\delta$ не разбивающая, то группа гомологий $\mathrm{H}_{2}\left(\Sigma^{*}, \mathbb{Z}\right)$ изоморфна $\mathbb{Z}$ и порождается образом класса $[\Sigma] \in \mathrm{H}_{2}(\Sigma, \mathbb{Z}) ;$ в противном случае группа $\mathrm{H}_{2}\left(\Sigma^{*}, \mathbb{Z}\right)$ изоморфна $\mathbb{Z} \oplus \mathbb{Z}$ и порождается фундаментальными ииклами двух неприводимых компонент слоя $\Sigma^{*}$. 
4) Группа гомологий $\mathrm{H}_{1}\left(\Sigma^{*}, \mathbb{Z}\right)$ является фактором группь $\mathrm{H}_{1}(\Sigma, \mathbb{Z})$ по подгруппе $\mathbb{Z}\langle[\delta]\rangle$, порожденной классом $[\delta]$, а группа когомологий $\mathrm{H}^{1}\left(\Sigma^{*}, \mathbb{Z}\right)$ есть ортогональный подмодуль $\mathbb{Z}\langle[\delta]\rangle^{\perp} \subset \mathrm{H}_{1}(\Sigma, \mathbb{Z})$ относительно естественного спаривания $\mathrm{H}_{1}(\Sigma, \mathbb{Z}) \times \mathrm{H}_{1}(\Sigma, \mathbb{Z}) \rightarrow \mathbb{Z}$.

5) Группь гомологий границы $\partial Z=Z_{\gamma}$ могут быть включены в точные последовательности

$$
\begin{gathered}
0 \longrightarrow \mathrm{H}_{0}\left(\gamma, \mathscr{H}_{1}\left(Z_{y}, \mathbb{Z}\right)\right) \longrightarrow \mathrm{H}_{1}\left(Z_{\gamma}, \mathbb{Z}\right) \longrightarrow \mathrm{H}_{1}\left(\gamma, \mathscr{H}_{0}\left(Z_{y}, \mathbb{Z}\right)\right) \longrightarrow 0, \\
0 \longrightarrow \mathrm{H}_{0}\left(\gamma, \mathscr{H}_{2}\left(Z_{y}, \mathbb{Z}\right)\right) \longrightarrow \mathrm{H}_{2}\left(Z_{\gamma}, \mathbb{Z}\right) \longrightarrow \mathrm{H}_{1}\left(\gamma, \mathscr{H}_{1}\left(Z_{y}, \mathbb{Z}\right)\right) \longrightarrow 0,
\end{gathered}
$$

где $\mathscr{H}_{p}\left(Z_{y}, \mathbb{Z}\right)$ обозначает локально постоянный пучок со слоем $\mathrm{H}_{p}\left(Z_{y}, \mathbb{Z}\right)$ над точкой $y \in \gamma$. В частности, имеются естественные изоморфизмы

$$
\mathrm{H}_{1}(\gamma, \mathbb{Z}) \stackrel{\cong}{\longrightarrow} \mathrm{H}_{1}\left(\gamma, \mathscr{H}_{0}\left(Z_{y}, \mathbb{Z}\right)\right), \quad \mathrm{H}_{0}\left(\gamma, \mathscr{H}_{2}\left(Z_{y}, \mathbb{Z}\right)\right) \stackrel{\cong}{\longrightarrow} \mathrm{H}_{2}(\Sigma, \mathbb{Z}),
$$

группа $\mathrm{H}_{1}\left(\gamma, \mathscr{H}_{1}\left(Z_{y}, \mathbb{Z}\right)\right)$ есть в точности подгруппа $[\delta]^{\perp}$ в $\mathrm{H}_{1}(\Sigma, \mathbb{Z})$, m. е. подгруппа, состоящая из таких $\lambda \in \mathrm{H}_{1}(\Sigma, \mathbb{Z})$, что $\lambda \cap[\delta]=0$, a $\mathrm{H}_{0}\left(\gamma, \mathscr{H}_{1}\left(Z_{y}, \mathbb{Z}\right)\right)$ есть факторгруппа группь $\mathrm{H}_{1}(\Sigma, \mathbb{Z})$ nо подгруппе $\mathbb{Z}\langle[\delta]\rangle$, порожденной клас$\cos [\delta]$.

Доказательства утверждений 1)-5) леммы 1.5 можно найти в [3]. Точные последовательности в части 5) получаются из спектральной последовательности Лерэ проекции $\mathrm{pr}: Z_{\gamma} \rightarrow \gamma$. Окружность $\delta$ называется исчезающим ииклом монодромии в точке $z^{*}$, а ее класс гомологий $[\delta] \in \mathrm{H}_{1}(\Sigma, \mathbb{Z})$ называется исчезающим классом.

\section{3. Гомотопический тип групп диффеоморфизмов поверхностей.} Пусть $\Sigma$ - замкнутая ориентированная поверхность рода $g=g(\Sigma)$ с отмеченной точкой $z_{0} \in \Sigma$. Обозначим через $\mathscr{D} i f f_{+}(\Sigma)$ группу сохраняющих ориентацию диффеоморфизмов поверхности $\Sigma$, через $\mathscr{D}$ iff $f_{+}\left(\Sigma, z_{0}\right)$ подгруппу диффеоморфизмов, сохраняющих отмеченную точку $z_{0}$, а через $\mathscr{D}$ iff $f_{+}\left(\Sigma,\left[z_{0}\right]\right)$ подгруппу диффеоморфизмов, сохраняющих точку $z_{0}$ и действующих тривиально в касательной плоскости $T_{z_{0}} \Sigma$. Обозначим через $\mathrm{Map}_{g}, \mathrm{Map}_{g, 1}$ и $\mathrm{Map}_{g,[1]}$ соответствующие группы классов отображений, т. е. группы связных компонент групп $\mathscr{D} i f f_{+}(\Sigma), \mathscr{D} i f f_{+}\left(\Sigma, z_{0}\right)$ и $\mathscr{D} i f f_{+}\left(\Sigma,\left[z_{0}\right]\right)$ соответственно. Заметим, что естественное действие группы $\mathscr{D} i f f_{+}(\Sigma)$ на поверхности $\Sigma$ определяет главное расслоение $\mathrm{ev}_{z_{0}}: \mathscr{D}$ iff $f_{+}(\Sigma) \rightarrow \Sigma$, задаваемое формулой $f \in \mathscr{D}$ iff $f_{+}(\Sigma) \mapsto f\left(z_{0}\right) \in \Sigma$, со структурной группой $\mathscr{D}$ iff $f_{+}\left(\Sigma, z_{0}\right)$. Таким образом, мы получаем длинную точную последовательность гомотопических групп

$$
\begin{gathered}
\cdots \rightarrow \pi_{k+1}(\Sigma) \stackrel{\partial}{\longrightarrow} \pi_{k}\left(\mathscr{D}_{i f f_{+}}\left(\Sigma, z_{0}\right)\right) \rightarrow \pi_{k}\left(\mathscr{D} i f f_{+}(\Sigma)\right) \rightarrow \pi_{k}(\Sigma) \stackrel{\partial}{\longrightarrow} \cdots \\
\cdots \rightarrow \pi_{1}(\Sigma) \stackrel{\partial_{\pi}}{\longrightarrow} \pi_{0}\left(\mathscr{D} i f f_{+}\left(\Sigma, z_{0}\right)\right) \rightarrow \pi_{0}\left(\mathscr{D} \text { iff } f_{+}(\Sigma)\right) \rightarrow 1 .
\end{gathered}
$$

Аналогично, естественное действие группы $\mathscr{D}$ iff $+\left(\Sigma, z_{0}\right)$ на плоскости $T_{z_{0}} \Sigma$ дифференциалами $D_{z_{0}} f: T_{z_{0}} \rightarrow T_{z_{0}}$ отображений $f \in \mathscr{D}$ iff $f_{+}\left(\Sigma, z_{0}\right)$ задает главное расслоение $D_{z_{0}}: \mathscr{D}$ iff $f_{+}\left(\Sigma, z_{0}\right) \rightarrow \boldsymbol{G} \boldsymbol{l}_{+}\left(T_{z_{0}} \Sigma\right)$ со структурной группой 
$\mathscr{D}$ if $f_{+}\left(\Sigma,\left[z_{0}\right]\right)$. Отсюда получается еще одна длинная точная последовательность

$$
\begin{gathered}
\cdots \rightarrow \pi_{k+1}\left(\boldsymbol{G} \boldsymbol{l}_{+}(2, \mathbb{R})\right) \stackrel{\partial}{\longrightarrow} \pi_{k}\left(\mathscr{D} \text { iff } f_{+}\left(\Sigma,\left[z_{0}\right]\right)\right) \rightarrow \pi_{k}\left(\mathscr{D} \text { iff } f_{+}\left(\Sigma, z_{0}\right)\right) \rightarrow \cdots \\
\cdots \rightarrow \pi_{1}\left(\boldsymbol{G l}_{+}(2, \mathbb{R})\right) \stackrel{\partial}{\longrightarrow} \pi_{0}\left(\mathscr{D} \text { iff } f_{+}\left(\Sigma,\left[z_{0}\right]\right)\right) \rightarrow \pi_{0}\left(\mathscr{D} \text { iff } f_{+}\left(\Sigma, z_{0}\right)\right) \rightarrow 1 .
\end{gathered}
$$

Следующие утверждения о группах классов отображений хорошо известны (см. [9] или [28]).

ПРЕДЛОЖЕНИЕ 1.6. і) Если $g=0$ или $g=1, \operatorname{mo}_{0}\left(\mathscr{D}\right.$ iff $\left.f_{+}\left(\Sigma, z_{0}\right)\right) \rightarrow$ $\pi_{0}\left(\mathscr{D i f f}_{+}(\Sigma)\right)$ является изоморфизмом.

ii) Eсли $g \geqslant 2$, то связная компонента единищы $\mathscr{D}$ iff $f_{0}(\Sigma)$ в группе $\mathscr{D}$ iff $f_{+}(\Sigma)$ стягиваема и последовательность (1.1) индуцирует точную последовательность грynn

$$
1 \rightarrow \pi_{1}(\Sigma) \stackrel{\partial_{\pi}}{\longrightarrow} \operatorname{Map}_{g, 1} \rightarrow \operatorname{Map}_{g} \rightarrow 1
$$

При этом группа Мар $_{g, 1}$ изоморфна подгруппе индекса 2 в группе $\operatorname{Aut}\left(\pi_{1}(\Sigma)\right)$, состоящей из тех автоморфизмов $\pi_{1}(\Sigma)$, которые тривиально действуют на группе $\mathrm{H}_{2}\left(\pi_{1}(\Sigma), \mathbb{Z}\right)$; отображение $\pi_{1}(\Sigma) \stackrel{\partial_{\pi}}{\longrightarrow}$ Map $_{g, 1}$ сопоставляет каждому элементу $\gamma \in \pi_{1}(\Sigma)$ внутренний автоморфизм $i_{\gamma}: \alpha \in \pi_{1}(\Sigma) \mapsto \gamma \alpha \gamma^{-1} \in \pi_{1}(\Sigma)$, а группа Map $_{g}$ есть соответствующая группа внешних автоморфизмов групnъь $\pi_{1}(\Sigma)$.

iii) Если $g \geqslant 1$, то точная последовательность (1.2) задает центральное расширение

$$
1 \rightarrow \mathbb{Z}=\pi_{1}\left(\boldsymbol{G l}_{+}(2, \mathbb{R})\right) \stackrel{\partial}{\longrightarrow} \operatorname{Map}_{g,[1]} \rightarrow \operatorname{Map}_{g, 1} \rightarrow 1
$$

iv) Имеются изоморфизмы $\pi_{1}\left(\mathscr{D}\right.$ iff $\left.f_{+}\left(S^{2}\right)\right) \cong \mathbb{Z}_{2}, \pi_{1}\left(\mathscr{D}\right.$ iff $\left.f_{+}\left(T^{2}\right)\right) \cong \pi_{1}\left(T^{2}\right) \cong \mathbb{Z}^{2}$.

Вложение $\pi_{1}\left(\Sigma, z_{0}\right) \stackrel{\partial_{\pi}}{\longrightarrow} \operatorname{Map}_{g, 1}$ (при $g \geqslant 2$ ) легко описать геометрически. А именно, для любого элемента $\gamma \in \pi_{1}\left(\Sigma, z_{0}\right)$ выберем изотопию $F_{t}: \Sigma \rightarrow \Sigma$, $t \in[0,1]$, такую, что $F_{0} \equiv \operatorname{id}_{\Sigma}$ и петля $[0,1] \ni t \mapsto F_{t}\left(z_{0}\right)$ представляет элемент $\gamma \in \pi_{1}\left(\Sigma, z_{0}\right)$. Из этого определения немедленно следует, что $F_{1}$ представляет образ $\partial_{\pi}(\gamma) \in \operatorname{Map}_{g, 1}$. В специальном случае, когда элемент $\gamma \in \pi_{1}\left(\Sigma, z_{0}\right)$ представлен гладко вложенной кривой, которую мы также обозначим через $\gamma$, можно дать еще более явное описание $\partial_{\pi}(\gamma)$. Для этого рассмотрим кривые $\gamma_{+}$ и $\gamma_{-}$, образующие границу трубчатой окрестности $U_{\gamma}$ кривой $\gamma$ на поверхности $\Sigma$. Тогда скручивания Дена $T_{\gamma_{ \pm}}$поверхности $\Sigma$ относительно кривых $\gamma_{ \pm}$ задают корректно определенные элементы группы Map $_{g, 1}$ и, как нетрудно проверить, выполнено соотношение

$$
\partial_{\pi}(\gamma)=T_{\gamma_{+}} \circ T_{\gamma_{-}}^{-1}
$$

Отсюда следует, что группа Мap $_{g, 1}$ порождается скручиваниями Дена относительно гладко вложенных кривых $\gamma$ на поверхности $\Sigma$, не проходящих через точку $z_{0}$. 
1.4. Гомологии семейств Лефшеца. Здесь мы рассматриваем условия, необходимые для гомологической тривиальности бутылки Клейна, вложенной в семейство Морса-Лефшеца $\mathrm{pr}: X \rightarrow Y$. Будем рассматривать ситуацию, несколько более общую, чем возникающая при применении леммы 1.3.

Пусть pr: $X \rightarrow Y$ - топологическое семейство Лефшеца с общим слоем $\Sigma$ над ориентированной поверхностью $Y$. Предположим, что $K \subset X-$ вложенная бутылка Клейна, удовлетворяющая следующим условиям:

$(\mathrm{T} 1)$ образ $\operatorname{pr}(K)$ - вложенная окружность $\Gamma \subset Y$, на которой нет критических значений проекции pr;

(Т2) ограничение проекции $\left.\mathrm{pr}\right|_{K}: K \rightarrow \Gamma$ - расслоение со слоем $S^{1}$.

Условия (Т1), (Т2) означают, что $(X, \mathrm{pr}, K, \Gamma)$ - семейство Морса-Лефшеца специального вида.

Отождествим поверхность $\Sigma$ со слоем $\mathrm{pr}^{-1}\left(y_{0}\right)$ над фиксированной точкой $y_{0} \in \Gamma$. Пусть $\boldsymbol{m}-$ слой $K$ над $y_{0}$, т. е. $\boldsymbol{m}=\Sigma \cap K$. Будем называть окружность $\boldsymbol{m}$ меридианом бутылки Клейна $K$, но рассматривать ее будем, в основном, как кривую на поверхности $\Sigma$. Пусть $F_{\Gamma}: \Sigma \rightarrow \Sigma-$ монодромия проекции $\mathrm{pr}: X_{\Gamma} \rightarrow \Gamma$. Напомним, что диффеоморфизм $F_{\Gamma}$ определен с точностью до изотопии. Выберем его так, что $F_{\Gamma}(\boldsymbol{m})=\boldsymbol{m}$.

Рассмотрим сначала один простой случай.

ПРЕДЛОЖЕНИЕ 1.7. Пусть $[\boldsymbol{m}]=0 \in \mathrm{H}_{1}\left(\Sigma, \mathbb{Z}_{2}\right)$, m. е. окружность $\boldsymbol{m}$ разбивает $\Sigma$. Тогда род $g$ слоя $\Sigma$ четен и бутылка Клейна $K$ гомологична слою $\Sigma=\operatorname{pr}^{-1}\left(y_{0}\right)$.

В условиях основной теоремы (после применения леммы 1.3) слой семейства Лефшеца заведомо не гомологичен нулю (над $\left.\mathbb{Z}_{2}\right)$, так как имеет индекс пересечения 1 с любым исключительным сечением. Тем самым, предложение 1.7 дает доказательство основной теоремы в (простом) частном случае, когда меридиан $\boldsymbol{m}$ гомологически тривиален в слое семейства Морса-Лефшеца для $(X, K)$. На самом деле, как будет показано в п. 2.3, слой не гомологичен нулю над $\mathbb{Z}_{2}$ для любого топологического семейства Лефшеца со слоем четного рода (см. теорему 2.30 и теорему 0.10$)$.

ДОКАЗАТЕЛЬСТВО ПРЕДЛОЖЕНИЯ 1.7. По условию меридиан $\boldsymbol{m}$ разбивает слой $\Sigma$ на две части, $\Sigma \backslash \boldsymbol{m}=\Sigma^{\prime} \sqcup \Sigma^{\prime \prime}$. Поскольку монодромия $F_{\Gamma}$ сохраняет ориентацию на $\Sigma$ и обращает ориентацию на $\boldsymbol{m}$, отображение $F_{\Gamma}$ должно менять местами $\Sigma^{\prime}$ и $\Sigma^{\prime \prime}$. Представляя $X_{\Gamma}$ как тор диффеоморфизма $F_{\Gamma}: \Sigma \rightarrow \Sigma$, мы видим, что граница $\mathbb{Z}_{2}$-цепи $\Sigma^{\prime} \times[0,1]$ в $X_{\Gamma}$ есть $[K]+[\Sigma]$. Кроме того, поверхности с краями $\Sigma^{\prime}$ и $\Sigma^{\prime \prime}$ имеют одинаковый род $g^{\prime}$, и потому род поверхности $\Sigma$ должен быть четным. Предложение доказано.

Перейдем теперь к рассмотрению случая, когда меридиан не гомологичен нулю в слое семейства Лефшеца. Обозначим через $\mu:=[\boldsymbol{m}] \in \mathrm{H}_{1}\left(\Sigma, \mathbb{Z}_{2}\right)$ гомологический класс меридиана и через $y_{i}^{*}, i=1, \ldots, n$, критические значения проекции $\mathrm{pr}: X \rightarrow Y$ (их может и не быть). Для каждой точки $y_{i}^{*}$ зафиксируем маленький диск $D_{i}$, содержащий $y_{i}^{*}$, и положим $\Gamma_{i}:=\partial D_{i}$. (Это вложенная кривая, обходящая вокруг точку $y_{i}^{*}$.) Обозначим $Y^{\circ}:=Y \backslash\left(\bigcup_{i} D_{i}\right)$. Ясно, что область $Y^{\circ}$ гомотопически эквивалентна дополнению к множеству критических значений. Напомним, что для любого подмножества $A \subset Y$ мы 
обозначаем через $X_{A}:=\operatorname{pr}^{-1}(A)$ часть многообразия $X$, лежащую над $A$. Множество $X_{Y^{\circ}}=\operatorname{pr}^{-1}\left(Y^{\circ}\right)$ обозначим через $X^{\circ}$. В случае разбивающей кривой $\Gamma$ обозначим через $Y_{+}$и $Y_{-}$соответствующие части базы $Y$, а через $g_{Y}^{+}$и $g_{Y}^{-}$их рода. Положим $Y_{ \pm}^{\circ}:=Y_{ \pm} \cap Y^{\circ}$ и $X_{ \pm}^{\circ}:=X_{Y_{ \pm}^{\circ}}=\operatorname{pr}^{-1}\left(Y_{ \pm}^{\circ}\right)$.

Гомологическая спектральная последовательность расслоения $\mathrm{pr}: X^{\circ} \rightarrow Y^{\circ}$ вырождается в члене $E_{p, q}^{2}$ и порождает точную последовательность

$$
0 \rightarrow \mathrm{H}_{0}\left(Y^{\circ}, \mathscr{H}_{2}\left(X_{y}, \mathbb{Z}_{2}\right)\right) \rightarrow \mathrm{H}_{2}\left(X^{\circ}, \mathbb{Z}_{2}\right) \rightarrow \mathrm{H}_{1}\left(Y^{\circ}, \mathscr{H}_{1}\left(X_{y}, \mathbb{Z}_{2}\right)\right) \rightarrow 0
$$

где $\mathscr{H}_{p}\left(X_{y}, \mathbb{Z}_{2}\right)$ - локально постоянный пучок со слоем $\mathrm{H}_{p}\left(X_{y}, \mathbb{Z}_{2}\right)$ в точке $y \in Y^{\circ}$. Поскольку отображение монодромии $F_{\Gamma}$ обращает ориентацию на кривой $\boldsymbol{m}$, случай $Y^{\circ}=S^{2}$ невозможен. Следовательно, поверхность $Y^{\circ}$ является $\pi_{1}$-пространством Эйленберга-Маклейна, и $\mathrm{H}_{p}\left(Y^{\circ}, \mathscr{H}_{q}\left(X_{y}, \mathbb{Z}_{2}\right)\right)=\mathrm{H}_{p}\left(\pi_{1}\left(Y^{\circ}\right)\right.$, $\left.\mathscr{H}_{q}\left(X_{y}, \mathbb{Z}_{2}\right)\right)$.

Для вычисления групп гомологий $\mathrm{H}_{p}\left(\pi_{1}\left(Y^{\circ}\right), M\right)$ с коэффициентами в заданном $\pi_{1}\left(Y^{\circ}\right)$-модуле $M$ мы воспользуемся специальным клеточным разбиением поверхности $Y^{\circ}$ и соответствующим ему копредставлением группы $\pi_{1}\left(Y^{\circ}\right)$. Пусть $g_{Y}-$ род поверхности $Y$. Наше построение зависит от того, разбивает кривая Г поверхность $Y$ или не разбивает.

В случае неразбивающей кривой $Г$ рассмотрим многоугольник $C_{Y}$ с $2 g_{Y}+n$ сторонами, где $n$ - число критических значений проекции $\mathrm{pr}: X \rightarrow Y$, т. е. число дыр в $Y^{\circ}$. Отметим и ориентируем стороны многоугольника $C_{Y}$ согласно соотношению

$$
R:=\left[\xi_{1}, \eta_{1}\right] \cdots\left[\xi_{g_{Y}}, \eta_{g_{Y}}\right] \cdot \Gamma_{1} \cdots \Gamma_{n} .
$$

Попарно склеивая первые $2 g_{Y}$ сторон многоугольника $C_{Y}$ согласно этой разметке и ориентациям, мы получаем поверхность $Y^{\prime}$ рода $g_{Y}$ с единственной граничной окружностью, разделенной на отрезки $\Gamma_{1}, \ldots, \Gamma_{n}$. Для того чтобы получить поверхность $Y^{\circ}$, мы делим каждый отрезок $\Gamma_{i}$ на три части, скажем $\Gamma_{i}^{\prime}, \Gamma_{i}^{\prime \prime}$ и $\Gamma_{i}^{\prime \prime \prime}$, и склеиваем отрезки $\Gamma_{i}^{\prime}$ и $\Gamma_{i}^{\prime \prime \prime}$ с обращением ориентации.

На построенной таким образом поверхности $Y^{\circ}$ все концы сторон $\xi_{i}, \eta_{i}, \Gamma_{j}$ отождествлены в одну точку. Возьмем эту точку в качестве базовой точки $y_{0}$ на $Y^{\circ}$ и получим нужное нам копредставление группы $\pi_{1}\left(Y^{\circ}, y_{0}\right)$ :

$$
\pi_{1}\left(Y^{\circ}, y_{0}\right)=\left\langle\xi_{1}, \eta_{1}, \ldots, \xi_{g_{Y}}, \eta_{g_{Y}} ; \Gamma_{1}, \ldots, \Gamma_{n} \mid\left[\xi_{1}, \eta_{1}\right] \cdots\left[\xi_{g_{Y}}, \eta_{g_{Y}}\right] \cdot \Gamma_{1} \cdots \Gamma_{n}=1\right\rangle .
$$

Здесь кривые $\xi_{i}, \eta_{i}, \Gamma_{j}$ отождествляются с соответствующими им элементами группы $\pi_{1}\left(Y^{\circ}, y_{0}\right)$. Кроме того, мы будем считать, что кривая $\Gamma$ - это кривая $\xi_{1}$.

В случае разбивающей кривой Г построение нужно модифицировать следующим образом. Занумеруем критические значения $y_{i}^{*}$ так, что первые $n^{+}$из них лежат в $Y_{+}$, а остальные $n^{-}:=n-n^{+}-$в $Y_{-}$. Положим

$$
\begin{gathered}
R^{+}:=\left[\xi_{1}, \eta_{1}\right] \cdots\left[\xi_{g_{Y}^{+}}, \eta_{g_{Y}^{+}}\right] \cdot \Gamma_{1} \cdots \Gamma_{n^{+}}, \\
R^{-}:=\left[\xi_{g_{Y}^{+}+1}, \eta_{g_{Y}^{+}+1}\right] \cdots\left[\xi_{g_{Y}}, \eta_{g_{Y}}\right] \cdot \Gamma_{n^{+}+1} \cdots \Gamma_{n} .
\end{gathered}
$$

Возьмем многоугольники $C_{Y}^{+}$и $C_{Y}^{-}$с $2 g_{Y}^{ \pm}+n^{ \pm}+1$ сторонами, отмеченными и ориентированными согласно соотношениям $R^{+} \cdot \Gamma^{+}$и $R^{-} \cdot \Gamma^{-}$соответственно. Склеим многоугольники $C_{Y}^{+}$и $C_{Y}^{-}$вдоль сторон $\Gamma^{+}$и $\Gamma^{-}$и обозначим через Г образованный этими сторонами отрезок. Получится многоугольник $C_{Y}$ 
с $2 g_{Y}+n$ сторонами, отмеченными и ориентированными согласно соотношению $R^{+} \cdot R^{-}$. Склеим поверхность $Y^{\circ}$ из многоугольника $C_{Y}$, как это было сделано выше. В качестве копредставления группы $\pi_{1}\left(Y^{\circ}\right)$ рассмотрим образующие и соотношения

$$
\pi_{1}\left(Y^{\circ}, y_{0}\right)=\left\langle\xi_{1}, \eta_{1}, \ldots, \xi_{g_{Y}}, \eta_{g_{Y}} ; \Gamma_{1}, \ldots, \Gamma_{n} ; \Gamma \mid R^{+}=\Gamma^{-1}, R^{-}=\Gamma\right\rangle .
$$

Заметим, что образы многоугольников $C_{Y}^{+}$и $C_{Y}^{-}$в поверхности $Y^{\circ}$ суть области $Y_{+}^{\circ}$ и $Y_{-}^{\circ}$, склеенные согласно соотношениям $R^{+} \cdot \Gamma^{+}$и $R^{-} \cdot \Gamma^{-}$соответственно. Мы видим, что фундаментальные группы $\pi_{1}\left(Y_{ \pm}^{\circ}\right)$ вкладываются в группу $\pi_{1}\left(Y^{\circ}\right)$ с помощью отождествления образующих.

Пусть теперь $\pi$ - произвольная группа, заданная копредставлением $\pi=$ $\langle\mathcal{X} \mid \mathcal{R}\rangle$, с множеством образующих $\mathcal{X}$ и множеством соотношений $\mathcal{R}$, и пусть $M-\pi$-модуль. Обозначим через $\operatorname{Fr}(\mathcal{X})$ свободную группу с образующими $\mathcal{X}$. Обозначим через $\mathcal{X} \otimes M$ прямую сумму " $\mathcal{X}$ экземпляров" $M$, так что элементы $\mathcal{X} \otimes M$ - это конечные формальные суммы вида $\sum_{i} x_{i} \otimes m_{i}$, где $x_{i} \in \mathcal{X}$ и $m_{i} \in M$, дистрибутивные по первому аргументу. Аналогичным образом определим $\mathcal{R} \otimes M$ и $\operatorname{Fr}(\mathcal{X}) \otimes M$. Будем рассматривать $\mathcal{X} \otimes M$ и $\mathcal{R} \otimes M$ как подгруппы в $\operatorname{Fr}(\mathcal{X}) \otimes M$, а чтобы различать два экземпляра $\operatorname{Fr}(\mathcal{X}) \otimes M$, будем использовать обозначения $x \otimes_{1} m$ и $x \otimes_{2} m$ соответственно. Положим $\mathscr{R}_{0}:=M, \mathscr{R}_{1}:=\mathcal{X} \otimes M$, $\mathscr{R}_{2}:=\mathcal{R} \otimes M$ и определим цепные гомоморфизмы $\partial_{1}: \mathscr{R}_{1} \rightarrow \mathscr{R}_{0}$ и $\partial_{2}: \mathscr{R}_{2} \rightarrow \mathscr{R}_{1}$ формулой $\partial_{1}\left(x \otimes_{1} m\right):=x m-m$ в первом случае и свойствами дифферениирования во втором:

1) $\partial_{2}\left(x \otimes_{2} m\right)=x \otimes_{1} m$, если $x \in \mathcal{X}$

2) $\partial_{2}\left(a b \otimes_{2} m\right)=\partial_{2}\left(a \otimes_{2} b m\right)+\partial_{2}\left(b \otimes_{2} m\right)$ для любых $a, b \in \operatorname{Fr}(\mathcal{X})$.

Существует единственный гомоморфизм $\partial_{2}: \mathscr{R}_{2} \rightarrow \mathscr{R}_{1}$, удовлетворяющий этим условиям. В частности, $\partial_{2}\left(1 \otimes_{2} m\right)=\partial_{2}\left(1 \cdot 1 \otimes_{2} m\right)=2 \partial_{2}\left(1 \otimes_{2} m\right)$, и потому $\partial_{2}\left(1 \otimes_{2} m\right)=0$ для единичного элемента $1 \in \operatorname{Fr}(\mathcal{X})$. Аналогично, $\partial_{2}\left(a^{-1} \otimes_{2} m\right)=$ $-\partial_{2}\left(a \otimes_{2} a^{-1} m\right)$.

Геометрический смысл этих гомоморфизмов состоит в следующем. Представление $\pi=\langle\mathcal{X} \mid \mathcal{R}\rangle$ позволяет построить ассоциированный двумерный клеточный комплекс $Z$ с единственной 0-мерной клеткой $*$ и одно- и двумерными клетками, пронумерованными элементами множеств $\mathcal{X}$ и $\mathcal{R}$. Построение комплекса $Z$ совпадает с построением поверхности $Y^{\circ}$, проведенным выше. Тогда $\mathscr{R}_{2} \stackrel{\partial_{2}}{\longrightarrow} \mathscr{R}_{1} \stackrel{\partial_{1}}{\longrightarrow} \mathscr{R}_{0} \rightarrow 0$ - цепной комплекс, ассоциированный с локально постоянной системой коэффициентов на $Z$, соответствующей модулю $M$. В частности, $\partial_{1} \circ \partial_{2}=0$. Кроме того, комплекс $Z$ будет 2-эквивалентен пространству Эйленберга-Маклейна $B(\pi, 1)$, и, следовательно, $p$-мерные группы гомологий цепного комплекса $\left(\mathscr{R} \bullet, \partial_{\bullet}\right)$ изоморфны группам гомологий $\mathrm{H}_{p}(\pi, M)$ при $p=0$ и $p=1$.

По построению поверхность $Y^{\circ}$ гомотопически эквивалентна двумерному клеточному комплексу $Z$, ассоциированному с двумя вышеуказанными копредставлениями группы $\pi_{1}\left(Y^{\circ}\right)$. В качестве модуля коэффициентов мы рассмотрим группу гомологий $\mathrm{H}_{1}\left(\Sigma, \mathbb{Z}_{2}\right)$. Образ класса гомологий $[K]$ при проекции $\mathrm{H}_{2}\left(X^{\circ}, \mathbb{Z}_{2}\right) \rightarrow \mathrm{H}_{1}\left(Y^{\circ}, \mathscr{H}_{1}\left(X_{y}, \mathbb{Z}_{2}\right)\right)$ описать легко: он представляется цепью $\Gamma \otimes_{1} \mu \in \mathscr{R}_{1}$. 
Опишем теперь образ группы $\mathrm{H}_{2}\left(X^{\circ}, \mathbb{Z}_{2}\right)$ в группе $\mathrm{H}_{2}\left(X, \mathbb{Z}_{2}\right)$. Для этого используем последовательность Майера-Вьеториса для разложения многообразия $X$ в объединение $X^{\circ}$ и $\bigsqcup_{i} X_{D_{i}}$. (Напомним, что $X_{D_{i}}=\operatorname{pr}^{-1}\left(D_{i}\right)$ и $X^{\circ} \cap X_{D_{i}}=X_{\Gamma_{i}}=\operatorname{pr}^{-1}\left(\Gamma_{i}\right)$.) Интересующий нас участок этой точной последовательности таков:

$$
\cdots \rightarrow \mathrm{H}_{2}\left(\bigsqcup_{i} X_{\Gamma_{i}}, \mathbb{Z}_{2}\right) \rightarrow \mathrm{H}_{2}\left(X^{\circ}, \mathbb{Z}_{2}\right) \oplus \mathrm{H}_{2}\left(\bigsqcup_{i} X_{D_{i}}, \mathbb{Z}_{2}\right) \rightarrow \mathrm{H}_{2}\left(X, \mathbb{Z}_{2}\right) \rightarrow \cdots
$$

ЛЕмма 1.8. Ядро гомоморфизма $\mathrm{H}_{2}\left(X^{\circ}, \mathbb{Z}_{2}\right) \rightarrow \mathrm{H}_{2}\left(X, \mathbb{Z}_{2}\right)$ порождается классами $\Lambda_{i}$, проекиии которьх на $\mathrm{H}_{1}\left(Y^{\circ}, \mathscr{H}_{1}\left(X_{y}, \mathbb{Z}_{2}\right)\right)$ суть классъь вида $\Gamma_{i} \otimes_{1} \lambda_{i}$, где $\lambda_{i} \in \mathrm{H}_{1}\left(X_{y}, \mathbb{Z}_{2}\right) \cap$-ортогональнь исчезающим циклам $\delta_{i}$.

Условие, налагаемое на $\lambda_{i}$, тривиально в случае $\left[\delta_{i}\right]=0 \in \mathrm{H}_{1}\left(X_{y}, \mathbb{Z}_{2}\right)$.

ДокАЗАтельство леммы 1.8. Очевидно, $\mathrm{H}_{2}\left(\bigsqcup_{i} X_{\Gamma_{i}}\right)=\bigoplus_{i} \mathrm{H}_{2}\left(X_{\Gamma_{i}}\right)$. Для каждого из прямых слагаемых, как и в случае $X^{\circ}$, гомологическая спектральная последовательность сводится к точной последовательности

$$
0 \rightarrow \mathrm{H}_{0}\left(\Gamma_{i}, \mathscr{H}_{2}\left(X_{y}, \mathbb{Z}_{2}\right)\right) \rightarrow \mathrm{H}_{2}\left(X_{\Gamma_{i}}, \mathbb{Z}_{2}\right) \rightarrow \mathrm{H}_{1}\left(\Gamma_{i}, \mathscr{H}_{1}\left(X_{y}, \mathbb{Z}_{2}\right)\right) \rightarrow 0 .
$$

Группа $\mathrm{H}_{0}\left(\Gamma_{i}, \mathscr{H}_{2}\left(X_{y}, \mathbb{Z}_{2}\right)\right)$ есть просто группа $\mathbb{Z}_{2}$, порожденная классом $[\Sigma]$. Поскольку $\pi_{1}\left(\Gamma_{i}\right)=\mathbb{Z}$, группа $\mathrm{H}_{1}\left(\Gamma_{i}, \mathscr{H}_{1}\left(X_{y}, \mathbb{Z}_{2}\right)\right)$ изоморфна $\mathrm{H}_{1}\left(\mathbb{Z}, M_{i}\right)$, где $M_{i}$ - группа $\mathrm{H}_{1}\left(X_{y}, \mathbb{Z}_{2}\right)$ с действием $\pi_{1}\left(\Gamma_{i}\right)=\mathbb{Z}$, заданным монодромией вдоль окружности $\Gamma_{i}$, которая есть скручивание Дена $T_{\delta_{i}}$ относительно исчезающего цикла $\delta_{i}$. Таким образом, $\mathrm{H}_{1}\left(\Gamma_{i}, \mathscr{H}_{1}\left(X_{y}, \mathbb{Z}_{2}\right)\right)$ - подпространство $\left[\delta_{i}\right]^{\perp}$, состоящее из классов $\lambda \in \mathrm{H}_{1}\left(X_{y}, \mathbb{Z}_{2}\right)$, которые $\cap$-ортогональны классу $\left[\delta_{i}\right]$. С другой стороны, группа $\mathrm{H}_{0}\left(\Gamma_{i}, \mathscr{H}_{2}\left(X_{y}, \mathbb{Z}_{2}\right)\right)$ изоморфна $\mathbb{Z}_{2}$ и порождена классом слоя, и потому композиция отображений

$$
\mathrm{H}_{0}\left(\Gamma_{i}, \mathscr{H}_{2}\left(X_{y}, \mathbb{Z}_{2}\right)\right) \rightarrow \mathrm{H}_{2}\left(X_{\Gamma_{i}}, \mathbb{Z}_{2}\right) \rightarrow \mathrm{H}_{2}\left(X_{D_{i}}, \mathbb{Z}_{2}\right)
$$

является вложением. Следовательно, последовательность (1.9) расщепляется, и группу $\mathrm{H}_{1}\left(\Gamma_{i}, \mathscr{H}_{1}\left(X_{y}, \mathbb{Z}_{2}\right)\right)$ можно рассматривать как подгруппу в $\mathrm{H}_{2}\left(X_{\Gamma_{i}}, \mathbb{Z}_{2}\right)$. Более того, образ группы $\mathrm{H}_{1}\left(\Gamma_{i}, \mathscr{H}_{1}\left(X_{y}, \mathbb{Z}_{2}\right)\right)$ в группе $\mathrm{H}_{2}\left(X_{D_{i}}, \mathbb{Z}_{2}\right)$ тривиален. Отсюда следует утверждение леммы.

Установим теперь необходимые условия для тривиальности класса $[K] \in$ $\mathrm{H}_{2}\left(X, \mathbb{Z}_{2}\right)$.

ПРЕДЛОЖЕНИЕ 1.9. Предположим, что класс $\mu=[\boldsymbol{m}]$ нетривиален, но проекция класса $[K]$ в группе $\mathrm{H}_{1}\left(Y^{\circ}, \mathscr{H}_{1}\left(X_{y}, \mathbb{Z}_{2}\right)\right)$ обращается в нуль.

i) $B$ случае разбивающей кривой $\Gamma$ существует такое разложение $\mu=$ $\mu_{+}+\mu_{-} \in \mathrm{H}_{1}\left(\Sigma, \mathbb{Z}_{2}\right)$, что действие фундаментальной группь $\pi_{1}\left(Y_{+}^{\circ}\right)$ (соответственно, $\left.\pi_{1}\left(Y_{-}^{\circ}\right)\right)$ преобразованиями монодромии сохраняет класс $\mu_{+}$(соответственно, $\left.\mu_{-}\right)$. В частности, оба класса $\mu_{+} u \mu_{-}$инвариантны относительно $F_{\Gamma}$.

ii) В случае неразбивающей кривой Г группа монодромии проекиии $\mathrm{pr}: X^{\circ} \rightarrow$ $Y^{\circ}$ сохраняет класс $\mu \in \mathrm{H}_{1}\left(\Sigma, \mathbb{Z}_{2}\right)$. Более того, существует такой класс $\nu \in \mathrm{H}_{1}\left(\Sigma, \mathbb{Z}_{2}\right)$, что $\mu=\left(1+F_{\eta_{1}}^{-1}\right) \nu$ для преобразования монодромии $F_{\eta_{1}}$ вдоль кривой $\eta_{1}$, причем класс $\nu$ инвариантен при монодромном действии остальнъхх выбранных нами образующих группь $\pi_{1}\left(Y^{\circ}\right)$. 
ДокАзАтЕльство. Для упрощения формул будем обозначать через $\xi_{i}, \eta_{i}, \Gamma_{j}$ и кривые на $Y^{\circ}$, и соответствующие элементы группы $\pi_{1}\left(Y^{\circ}\right)$, и образующие свободной группы, и преобразования монодромии вдоль этих кривых.

i) Из леммы 1.8 следует, что элемент $\Gamma \otimes_{1} \mu$ может быть представлен в виде

$$
\Gamma \otimes_{1} \mu=\partial_{2}\left(R^{+} \cdot \Gamma \otimes_{2} \mu_{+}\right)+\partial_{2}\left(R^{-} \cdot \Gamma^{-1} \otimes_{2} \mu_{-}\right)+\sum_{i} \Gamma_{i} \otimes_{1} \lambda_{i}
$$

где $\mu_{ \pm}, \lambda_{i} \in \mathrm{H}_{1}\left(\Sigma, \mathbb{Z}_{2}\right)$, причем $\lambda_{i} \cap$-ортогональны циклам $\delta_{i}$. Распишем $\partial_{2}\left(R^{+} \cdot \Gamma \otimes_{2} \mu_{+}\right)$, сохраняя коммутаторы $\left[\xi_{i}, \eta_{i}\right]$, и обозначим через $w_{i}^{\Gamma}$ конечный фрагмент слова $R^{+} \cdot \Gamma$ после буквы $\Gamma_{i}$, а через $w_{i}^{[]}-$конечный фрагмент слова $R^{+} \cdot \Gamma$ после коммутатора $\left[\xi_{i}, \eta_{i}\right]$. В частности, $w_{n^{+}}^{\Gamma}=\Gamma, w_{n^{+-1}}^{\Gamma}=\Gamma_{n^{+}} \cdot \Gamma$, $w_{1}^{\Gamma}=\Gamma_{2} \cdots \Gamma_{n^{+}} \cdot \Gamma$, и аналогичное выражение имеется для $w_{i}^{[]}$. Отсюда получается, что

$$
\begin{aligned}
\partial_{2}\left(R^{+} \cdot \Gamma \otimes_{2} \mu_{+}\right)=\partial_{2}\left(\left[\xi_{1}, \eta_{1}\right] \cdots \Gamma_{n^{+}-1} \cdot \Gamma_{n^{+}} \cdot \Gamma \otimes_{2} \mu_{+}\right) \\
=\Gamma \otimes_{1} \mu_{+}+\Gamma_{n^{+}} \otimes_{1} \Gamma \mu_{+}+\Gamma_{n^{+-1}} \otimes_{1} \Gamma_{n^{+}} \cdot \Gamma \mu_{+}+\cdots+\Gamma_{1} \otimes_{1} w_{1}^{\Gamma} \cdot \mu_{+} \\
\quad+\partial_{2}\left(\left[\xi_{g_{Y}^{+}}, \eta_{g_{Y}^{+}}\right] \otimes_{2} w_{g_{Y}^{+}}^{[]} \mu_{+}\right)+\partial_{2}\left(\left[\xi_{g_{Y}^{+}-1}, \eta_{g_{Y}^{+}-1}\right] \otimes_{2} w_{g_{Y}^{+}-1}^{[]} \mu_{+}\right)+\cdots \\
\quad \cdots+\partial_{2}\left(\left[\xi_{1}, \eta_{1}\right] \otimes_{2} w_{1}^{[]} \mu_{+}\right) .
\end{aligned}
$$

Аналогичное выражение получается и для $\partial_{2}\left(R^{-} \cdot \Gamma^{-1} \otimes_{2} \mu_{-}\right)$, с тем отличием, что первое слагаемое равно $-\Gamma \otimes_{2} \Gamma^{-1} \mu_{-}$. Разложение коммутаторов дает формулу

$$
\begin{aligned}
\partial_{2}\left([\xi, \eta] \otimes_{2} \nu\right) & =\partial_{2}\left(\xi \eta \xi^{-1} \eta^{-1} \otimes_{2} \nu\right) \\
& =\xi \otimes_{1} \eta \xi^{-1} \eta^{-1} \nu+\eta \otimes_{1} \xi^{-1} \eta^{-1} \nu-\xi \otimes_{1} \xi^{-1} \eta^{-1} \nu-\eta \otimes_{1} \eta^{-1} \nu \\
& =\xi \otimes_{1}(\eta-1) \xi^{-1} \eta^{-1} \nu+\eta \otimes_{1}(1-\xi) \xi^{-1} \eta^{-1} \nu
\end{aligned}
$$

Приводя подобные члены (и игнорируя знаки, так как мы работаем над $\mathbb{Z}_{2}$ ), мы получаем требуемое разложение $\mu=\mu_{+}+\Gamma^{-1} \mu_{-}$из коэффициента при $\Gamma$, равенство $\lambda_{i}=w_{i}^{\Gamma} \mu_{ \pm}$из коэффициента при $\Gamma_{i}$, а равенства $\left(\eta_{i}-1\right) \xi_{i}^{-1} \eta_{i}^{-1} w_{i}^{[]} \mu_{ \pm}=0$ и $\left(1-\xi_{i}\right) \xi_{i}^{-1} \eta_{i}^{-1} w_{i}^{[]} \mu_{ \pm}=0$ из коэффициентов при $\xi_{i}$ и $\eta_{i}$. Заметим теперь, что равенство $\lambda_{i}=w_{i}^{\Gamma} \mu_{ \pm}$вместе с $\cap$-ортогональностью $\lambda_{i} \cap \delta_{i} \equiv 0 \bmod 2$ влечет инвариантность класса $w_{i}^{\Gamma} \mu_{ \pm}$при действии скручивания Дена $T_{\delta_{i}}$, т. е. при действии $\Gamma_{i}$. Отсюда следует тождество $w_{0}^{\Gamma} \mu_{+}=w_{1}^{\Gamma} \mu_{+}=\cdots=\Gamma \mu_{+}$для всех $i=0 ; 1, \ldots, n^{+}$. (Мы положили $w_{0}^{\Gamma}:=\Gamma_{1} \cdot w_{1}^{\Gamma}$; этот элемент совпадает с последним из $w_{g_{Y}^{+}}^{[]}$.)

Тривиальность действий $\xi_{i}$ и $\eta_{i}$ доказывается аналогично. Действительно, полученные выше равенства означают, что $\xi_{i}$ и $\eta_{i}$ тривиально действуют на $\xi_{i}^{-1} \eta_{i}^{-1} w_{i}^{[]} \mu_{+}$, так что $w_{i}^{[]} \mu_{+}$также остается инвариантным, откуда мы заключаем, что $w_{i-1}^{[]} \mu_{+}=w_{i}^{[]} \mu_{+}$. В итоге мы получаем $\pi_{1}\left(Y_{+}^{\circ}\right)$-инвариантность класса $\Gamma \mu_{+}$. Наконец, поскольку элемент $\Gamma$ в группе $\pi_{1}\left(Y_{+}^{\circ}\right)$ может быть представлен в виде произведения уже рассмотренных элементов, то $\Gamma \mu_{+}=\mu_{+}$. Доказательство для $\mu_{-}$точно такое же. 
ii) В этом случае $\Gamma=\xi_{1}$ в группе $\pi_{1}\left(Y^{\circ}\right)$ и неразложенное выражение имеет вид

$$
\xi_{1} \otimes_{1} \mu=\partial_{2}\left(R \otimes_{2} \nu\right)+\sum_{i} \Gamma_{i} \otimes_{1} \lambda_{i}
$$

Из него получаются те же формулы, что и в предыдущем случае, за исключением выражения для коэффициента при $\xi_{1}$, который равен $\mu+\left(\eta_{i}-1\right) \xi_{i}^{-1} \eta_{i}^{-1} w_{1}^{[]} \nu$. Точно так же получается инвариантность класса $\nu$ при действиях $\xi_{i}, \eta_{i}$ для всех $i=2, \ldots, n$ и при действиях всех $\Gamma_{i}$. Остается использовать равенства $\xi_{1}^{-1} \eta_{1}^{-1} \nu=\eta_{1}^{-1} \nu$ и $\left(1+\eta_{1}\right) \xi_{1}^{-1} \eta_{1}^{-1} \nu=\mu$. Поскольку коммутатор $\left[\xi_{1}, \eta_{1}\right]$ в группе $\pi_{1}\left(Y^{\circ}\right)$ равен выражению от прочих образующих, действия $\xi_{1}$ и $\eta_{1}$ на класс $\nu$ перестановочны. Поэтому первое равенство эквивалентно $\xi_{1}$-инвариантности класса $\nu$, а из второго следует формула $\left(1+\eta_{1}^{-1}\right) \nu=\mu$, что и требовалось доказать.

Лемма 1.10. Первая группа гомологий многообразия X включается в точную последовательность

$$
0 \rightarrow \mathrm{H}_{0}\left(Y^{\circ}, \mathscr{H}_{1}\left(X_{y}, \mathbb{Z}_{2}\right)\right) \rightarrow \mathrm{H}_{1}\left(X, \mathbb{Z}_{2}\right) \rightarrow \mathrm{H}_{1}\left(Y, \mathbb{Z}_{2}\right) \rightarrow 0
$$

ДокАзАтЕльство. Утверждение тривиально в случае, когда $Y=S^{2}$ и проекция pr: $X \rightarrow Y=S^{2}$ не имеет критических значений. Рассмотрим гомологии многообразия $X^{\circ}$ в остальных случаях. Спектральная последовательность Лерэ сводится к точной последовательности

$$
0 \rightarrow \mathrm{H}_{0}\left(Y^{\circ}, \mathscr{H}_{1}\left(X_{y}, \mathbb{Z}_{2}\right)\right) \rightarrow \mathrm{H}_{1}\left(X^{\circ}, \mathbb{Z}_{2}\right) \rightarrow \mathrm{H}_{1}\left(Y^{\circ}, \mathbb{Z}_{2}\right) \rightarrow 0
$$

Чтобы получить (1.11), используем последовательность Майера-Вьеториса, соответствующую покрытию $X=X^{\circ} \cup\left(\bigsqcup_{i} X_{D_{i}}\right)$, где $X^{\circ} \cap\left(\bigsqcup_{i} X_{D_{i}}\right)=\bigsqcup_{i} X_{\Gamma_{i}}$. Это дает отождествление группы $\mathrm{H}_{1}\left(X, \mathbb{Z}_{2}\right)$ с коядром гомоморфизма

$$
\bigoplus_{i} \mathrm{H}_{1}\left(X_{\Gamma_{i}}, \mathbb{Z}_{2}\right) \rightarrow \mathrm{H}_{1}\left(X^{\circ}, \mathbb{Z}_{2}\right) \oplus \bigoplus_{i} \mathrm{H}_{1}\left(X_{D_{i}}, \mathbb{Z}_{2}\right)
$$

В свою очередь, для каждого $X_{\Gamma_{i}}$ мы имеем последовательность

$$
0 \rightarrow \mathrm{H}_{0}\left(\Gamma_{i}, \mathscr{H}_{1}\left(X_{y}, \mathbb{Z}_{2}\right)\right) \rightarrow \mathrm{H}_{1}\left(X_{\Gamma_{i}}, \mathbb{Z}_{2}\right) \rightarrow \mathrm{H}_{1}\left(\Gamma_{i}, \mathbb{Z}_{2}\right) \rightarrow 0
$$

Заметим теперь, что вложение $X_{\Gamma_{i}} \subset X_{D_{i}}$ индуцирует изоморфизм

$$
\mathrm{H}_{0}\left(\Gamma_{i}, \mathscr{H}_{1}\left(X_{y}, \mathbb{Z}_{2}\right)\right) \stackrel{\cong}{\longrightarrow} \mathrm{H}_{1}\left(X_{D_{i}}, \mathbb{Z}_{2}\right)
$$

и, значит, дает естественное расщепление последовательности (1.14). "Подставляя" это расщепление в (1.13), мы видим, что каждая группа $\mathrm{H}_{1}\left(\Gamma_{i}, \mathbb{Z}_{2}\right) \cong \mathbb{Z}_{2}$ "убивает" тот класс в группе $\mathrm{H}_{1}\left(Y^{\circ}, \mathbb{Z}_{2}\right)$ из последовательности $(1.12)$, который представлен соответствующей кривой $\Gamma_{i} \subset Y$. 
1.5. $\mathbb{Z}_{2}$-сечения семейств Лефшеца. Если в условиях предложения 1.9 бутылка Клейна $K$ гомологически нетривиальна, то она должна быть $\mathbb{Z}_{2}$-гомологична слою $\mathrm{pr}^{-1}\left(y_{0}\right)$. (Эта же ситуация возникала выше в предложении 1.7.) В свою очередь, гомологическая нетривиальность слоя эквивалентна существованию класса $[\sigma] \in \mathrm{H}_{2}\left(X, \mathbb{Z}_{2}\right)$, для которого $[\sigma] \cap[K] \neq 0$. В настоящем пункте мы изучаем свойства таких классов $[\sigma]$. Эти результаты используются в доказательстве сформулированной во введении теоремы 0.10 .

Сохраним обозначения из п. 1.4 с небольшим изменением. В частности, $Y$ компактная поверхность с границей $\partial Y$, возможно, пустой или несвязной, а pr: $X \rightarrow Y$ - семейство Лефшеца со слоем $\Sigma=\operatorname{pr}^{-1}\left(y_{0}\right)$ рода $g$ такое, что критические значения $y_{i}^{*}$ не лежат на границе $\partial Y$. Обозначим через $Y^{\circ}$ поверхность, полученную из $Y^{\circ}$ удалением попарно непересекающихся дисков $D_{i}$ с центрами в точках $y_{i}^{*}$. Тогда ограничение проекции $\mathrm{pr}: \partial X \rightarrow \partial Y$ будет расслоением со слоем $\Sigma$.

ОПРеДЕЛЕНИЕ 1.11. $\mathbb{Z}_{2}$-сечением семейства $\mathrm{pr}: X \rightarrow Y$ называется относительный класс гомологий $\sigma \in \mathrm{H}_{2}\left(X, \partial X ; \mathbb{Z}_{2}\right)$, имеющий ненулевой $\mathbb{Z}_{2}$-индекс пересечения с классом $[\Sigma]$. Обозначим через $\partial \sigma \in \mathrm{H}_{1}\left(\partial X, \mathbb{Z}_{2}\right)$ класс границы $\sigma$, а через $\sigma^{\vee} \in \mathrm{H}^{2}\left(X, \mathbb{Z}_{2}\right)$ двойственный по Пуанкаре класс когомологий. Для открытого множества $U \subset Y$ с гладкой границей $\partial U$, на которой нет критических значений $y_{i}^{*}$, определим ограничение $\sigma$ на прообраз $X_{U}=\operatorname{pr}^{-1}(U)$ с помощью ограничения класса $\sigma^{\vee}$, т. е. $\left(\left.\sigma\right|_{X_{U}}\right)^{\vee}:=\left.\sigma^{\vee}\right|_{X_{U}}$.

Последнее определение можно также использовать для определения $\partial \sigma$, поскольку $(\partial \sigma)^{\vee}=\left.\sigma^{\vee}\right|_{\partial X}$.

Группа когомологий $\mathrm{H}^{2}\left(X, \mathbb{Z}_{2}\right)$, в которой лежит $\sigma^{\vee}$, имеет описание, двойственное к описанию группы гомологий $\mathrm{H}^{2}\left(X, \mathbb{Z}_{2}\right)$. В частности, имеется точная последовательность

$$
0 \rightarrow \mathrm{H}^{1}\left(Y^{\circ}, \mathscr{H}^{1}\left(X_{y}, \mathbb{Z}_{2}\right)\right) \rightarrow \mathrm{H}^{2}\left(X^{\circ}, \mathbb{Z}_{2}\right) \rightarrow \mathrm{H}^{0}\left(Y^{\circ}, \mathscr{H}^{2}\left(X_{y}, \mathbb{Z}_{2}\right)\right) \rightarrow 0,
$$

где через $\mathscr{H}^{p}\left(X_{y}, \mathbb{Z}_{2}\right)$ обозначен локально постоянный пучок со слоем $\mathrm{H}^{p}\left(X_{y}, \mathbb{Z}_{2}\right)$ в точке $y \in Y^{\circ}$, группа $\mathrm{H}^{0}\left(Y^{\circ}, \mathscr{H}^{2}\left(X_{y}, \mathbb{Z}_{2}\right)\right)$ естественно изоморфна $\mathbb{Z}_{2}\langle[\Sigma]\rangle$, а группа $\mathrm{H}^{1}\left(Y^{\circ}, \mathscr{H}^{1}\left(X_{y}, \mathbb{Z}_{2}\right)\right)$ равна $\mathrm{H}^{1}\left(\pi_{1}\left(Y^{\circ}\right), \mathscr{H}^{1}\left(X_{y}, \mathbb{Z}_{2}\right)\right)$. Кроме того, для любого $\pi_{1}\left(Y^{\circ}\right)$-модуля $M$ мы можем вычислить группы $\mathrm{H}^{1}\left(\pi_{1}\left(Y^{\circ}\right), M\right)$, используя резольвенту $0 \rightarrow \mathscr{R}^{0} \stackrel{d^{(1)}}{\longrightarrow} \mathscr{R}^{1} \stackrel{d^{(2)}}{\longrightarrow} \mathscr{R}^{2} \rightarrow 0$, двойственную резольвенте $\left(\mathscr{R}_{\bullet}, \partial_{\bullet}\right)$ из п. 1.4. В случае конечного копредставления $\langle\mathcal{X} \mid \mathcal{R}\rangle$ группы $\pi_{1}\left(Y^{\circ}\right)$ группы коцепей $\mathscr{R}^{\bullet}$ совпадают с соответствующими группами цепей, так что $\mathscr{R}^{0}=M, \mathscr{R}^{1}=\mathcal{X} \otimes^{1} M$ и $\mathscr{R}^{2}=\mathcal{R} \otimes^{2} M$, где индекс в обозначении $\otimes^{k}$ указывает, что элемент лежит в $\mathscr{R}^{k}$. Дифференциалы $d^{\bullet}$ двойственны дифференциалам $\partial$. и задаются транспонированными матрицами. Немедленно получается формула $d^{(1)}: m \mapsto \sum_{i} x_{i} \otimes^{1}\left(x_{i}-1\right) m$ для дифференциала $d^{(1)}: \mathscr{R}^{0}=M \rightarrow \mathscr{R}^{1}$. Формальное выражение для $d^{(2)}: \mathscr{R}^{1} \rightarrow \mathscr{R}^{2}$ имеет вид

$$
d^{(2)}\left(\sum_{i} x_{i} \otimes^{1} m_{i}\right)=\sum_{i, j} R_{j} \otimes^{2} \frac{\partial R_{j}}{\partial x_{i}} m_{i}
$$

где суммирование ведется по всем $x_{i} \in \mathcal{X}$ и $R_{j} \in \mathcal{R}$, а $\frac{\partial R_{j}}{\partial x_{i}}$ обозначает производную Фокса слова $R_{j}$ в свободной группе $\operatorname{Fr}(\mathcal{X})$, порожденной $\mathcal{X}$ (см. [9]). Для 
данного соотношения $R_{j}$ коэффициент $\sum_{i} \frac{\partial R_{j}}{\partial x_{i}} m_{i}$ равен значению на $R_{j} \in \operatorname{Fr}(\mathcal{X})$ единственного скрещенного гомоморфизма $\phi: \operatorname{Fr}(\mathcal{X}) \rightarrow M$ такого, что $\phi\left(x_{i}\right)=$ $m_{i}$. Напомним, что скрещенные гомоморфизмы характеризуются свойством $\phi(a \cdot b)=\phi(a)+a \cdot \phi(b)$ для всех $a, b \in \operatorname{Fr}(\mathcal{X})$, которое двойственно правилу Лейбница для дифференциала $\partial_{2}$.

При $g \geqslant 2$ обозначим через $\widehat{\operatorname{Map}}_{g}$ группу в расширении

$$
1 \rightarrow \mathrm{H}_{1}\left(\Sigma, \mathbb{Z}_{2}\right) \rightarrow{\widetilde{\operatorname{Map}_{g}}}^{\rightarrow \operatorname{Map}_{g} \rightarrow 1}
$$

так что $\widetilde{\operatorname{Map}}_{g}$ есть фактор группы $\mathrm{Map}_{g, 1}$ по образу ядра гомоморфизма $\pi_{1}\left(\Sigma, z_{0}\right) \rightarrow \mathrm{H}_{1}\left(\Sigma, \mathbb{Z}_{2}\right)$ при отображении $\partial_{\pi}$. Скручиванием Дена в группе $\widetilde{\mathrm{Map}}_{g}$ называется образ скручивания Дена $T_{\delta} \in \operatorname{Map}_{g, 1}$.

Лемма 1.12. Пусть pr: $X \rightarrow Y$-семейство Лефшеца на замкнутом многообразии $X$ и $\left\langle\xi_{j}, \eta_{j}, \Gamma_{i} \mid R\right\rangle$ - рассматривавшееся выше копредставление фундаментальной группы $\pi_{1}\left(Y^{\circ}\right)$ с единственным соотношением.

i) Образ гомоморфизма $\mathrm{H}^{2}\left(X, \mathbb{Z}_{2}\right) \rightarrow \mathrm{H}^{2}\left(X^{\circ}, \mathbb{Z}_{2}\right)$ порождается классом $\mathbb{Z}_{2}$-сечения (если такое существует) и классами из $\mathrm{H}^{1}\left(Y^{\circ}, \mathscr{H}^{1}\left(X_{y}, \mathbb{Z}_{2}\right)\right)$, представленными коциклами $\boldsymbol{\lambda}^{\vee}$ вида

$$
\lambda^{\vee}=\sum_{j}\left(\xi_{j} \otimes^{1} \lambda_{j}^{\vee}+\eta_{j} \otimes^{1} \mu_{j}^{\vee}\right)+\sum_{i} \Gamma_{i} \otimes^{1} n_{i} \delta_{i}^{\vee} \in \mathrm{H}^{1}\left(Y^{\circ}, \mathscr{H}^{1}\left(X_{y}, \mathbb{Z}_{2}\right)\right),
$$

где $n_{i} \in \mathbb{Z}_{2}, \lambda_{j}^{\vee}, \mu_{j}^{\vee} \in \mathrm{H}^{1}\left(\Sigma, \mathbb{Z}_{2}\right)$, а $\delta_{i}^{\vee}-\kappa л а с с$, двойственный по Пуанкаре клас$c y\left[\delta_{i}\right]$.

ii) Препятствие $\kappa$ существованию $\mathbb{Z}_{2}$-сечения семейства $\mathrm{pr}: X \rightarrow Y$ есть смежсный класс $\left[\vartheta^{\vee}\right]$ в факторе группы $\mathscr{R}^{2}$ по подгруппе всех когрании, $d^{(2)}\left(\boldsymbol{\lambda}^{\vee}\right)$, где $\boldsymbol{\lambda}^{\vee}$ имеет вид (1.16).

iii) В случае $g \geqslant 2$ препятствие $\left[\vartheta^{\vee}\right] \in \mathscr{R}^{2} /\left\{d^{(2)}\left(\boldsymbol{\lambda}^{\vee}\right)\right\}$ обращается в нуль тогда и только тогда, когда монодромия $\mathcal{F}: \pi_{1}\left(Y^{\circ}\right) \rightarrow$ Map $_{g}$ расслоения $\mathrm{pr}: X^{\circ} \rightarrow$ $Y^{\circ}$ поднимается до гомоморфизма $\widetilde{\mathcal{F}}: \pi_{1}\left(Y^{\circ}\right) \rightarrow \widetilde{\operatorname{Map}}_{g}$, при котором $\widetilde{\mathcal{F}}\left(\Gamma_{i}\right)-$ скручивание Дена в группе $\widetilde{\operatorname{Map}}_{g}$. Два таких подвема $\widetilde{\mathcal{F}}_{1}, \widetilde{\mathcal{F}}_{2}: \pi_{1}\left(Y^{\circ}\right) \rightarrow \widetilde{\operatorname{Map}}_{g}$ отличаются на скрещенный гомоморфизм $\phi: \pi_{1}\left(Y^{\circ}\right) \rightarrow \mathrm{H}_{1}\left(\Sigma_{1}\right)$, ассочиированный с единственной коцепъю $\boldsymbol{\lambda}_{\phi}^{\vee}$ вида (1.16), для которой двойственный по Пуанкаре класс $\boldsymbol{\lambda}_{\phi}$ имеет коэфбициенты $\lambda_{j}=\phi\left(\xi_{j}\right), \mu_{j}=\phi\left(\eta_{j}\right)$ u $n_{i} \delta_{i}=\phi\left(\Gamma_{i}\right)$.

ДокАЗАтельство. Утверждения і), ii) двойственны лемме 1.8, так что доказательство получается дуализацией гомоморфизма с учетом соотношений между образами и ядрами. Утверждение іiі) следует из того, что естественное вложение $\partial_{\pi}: \pi_{1}\left(\Sigma, z_{0}\right) \hookrightarrow \operatorname{Map}\left(\Sigma, z_{0}\right)$ при $g \geqslant 2$ индуцирует вложение $\mathrm{H}_{1}\left(\Sigma, \mathbb{Z}_{2}\right) \hookrightarrow \widehat{\mathrm{Map}_{g}}$. Остается заметить, что на уровне гомологий групп оба построения эквивалентны.

Следует иметь в виду, что при $g=1$ препятствие $\left[\vartheta^{\vee}\right]$ к существованию $\mathbb{Z}_{2}$-сечения не определяется монодромией в группе $\mathrm{Map}_{1}=\mathrm{Map}_{1,1}$. Это отражает тот факт, что гомоморфизм $\partial_{\pi}: \pi_{1}\left(T^{2}\right)=\mathbb{Z}^{2} \rightarrow \pi_{0}\left(\mathscr{D} i f f_{+}\left(T^{2}\right)\right)$ в последовательности (1.1) в этом случае тривиален. 
1.6. Пример. Здесь мы построим лагранжево вложение бутылки Клейна в проективную алгебраическую поверхность $X$ с кэлеровой формой $\omega$, для которого гомологический класс $[K]$ тривиален. В этом примере бутылка Клейна $K$ расслоена над неразбивающей кривой в торе $T^{2}$.

Пусть $Y$ - это тор $T^{2}$. Снабдим $Y$ плоской метрикой. Это задаст комплексную структуру и кэлерову форму $\omega_{Y}$ на торе $Y$. Будем предполагать, что объем $Y$ относительно формы $\omega_{Y}$ равен 1 . Зафиксируем геометрический базис тора $Y$, состоящий из вложенных окружностей $\xi$ и $\eta$, трансверсально пересекающихся в единственной точке.

Пусть $\Sigma$ - другой тор $T^{2}$, представленный как фактор комплексной прямой $\mathbb{C}$ по решетке $\Lambda$, натянутой на векторы $\alpha:=1$ и $\beta:=e^{2 \pi i / 3}$. Пусть $\omega_{\Sigma}-$ плоская кэлерова форма на $\Sigma$ объема 1 . Рассмотрим $\mathbb{C}$-линейные гомоморфизмы $F_{\xi}:=$ $-\mathrm{id}: \mathbb{C} \rightarrow \mathbb{C}$ и $F_{\eta}:=e^{\pi i / 3} \mathrm{id}: \mathbb{C} \rightarrow \mathbb{C}$. Ясно, что они определяют автоморфизмы тора $\Sigma$, оставляющие неподвижной базисную точку $z_{0}:=0 \in \Sigma$.

Пусть pr: $X \rightarrow Y$ - расслоение с базой $Y$ и слоем $\Sigma$, монодромия которого вдоль $\xi$ и $\eta$ равна $F_{\xi}$ и $F_{\eta}$ соответственно. Тогда существует плоская кэлерова структура на комплексной поверхности $X$ с кэлеровой формой $\omega$, которая в окрестности каждого слоя есть произведение $\left(Y, \omega_{Y}\right) \times\left(\Sigma, \omega_{\Sigma}\right)$.

Мы утверждаем, что $\omega$ задает поляризацию поверхности $X$, соответствующую некоторому голоморфному линейному расслоению $\mathscr{L}$. Действительно, поскольку монодромия сохраняет точку $z_{0} \in \Sigma$, мы получаем голоморфное сечение $\sigma_{0}$, тождественно равное $z_{0}$. Нетрудно видеть, что форма $\omega$ представляет первый класс Черна $c_{1}(\mathscr{L})$ голоморфного линейного расслоения $\mathscr{L}:=\mathscr{O}_{X}\left(\sigma_{0}+X_{y}\right)$, соответствующего дивизору $\sigma_{0}+X_{y}$, где $X_{y}-$ вертикальный слой над какой-либо точкой $y \in Y$. По теореме Кодаиры о вложении (см., например, [26]) расслоение $\mathscr{L}$ обильно и поверхность $X$ проективна.

Теперь вычислим группу $\mathrm{H}_{2}\left(X, \mathbb{Z}_{2}\right)$ с помощью спектральной последовательности. Ее член $E_{p, q}^{2}$ состоит из групп $\mathrm{H}_{p}\left(Y, \mathscr{H}_{q}\left(X_{y}, \mathbb{Z}_{2}\right)\right)$. Обе группы $\mathrm{H}_{0}\left(Y, \mathscr{H}_{2}\left(X_{y}, \mathbb{Z}_{2}\right)\right)$ и $\mathrm{H}_{2}\left(Y, \mathscr{H}_{0}\left(X_{y}, \mathbb{Z}_{2}\right)\right)$ изоморфны $\mathbb{Z}_{2}$ и порождены классом слоя $X_{y}$ и сечения $\sigma_{0}$ соответственно. Для вычисления $\mathrm{H}_{1}\left(Y, \mathscr{H}_{1}\left(X_{y}, \mathbb{Z}_{2}\right)\right)$ заметим, что эта группа есть модуль коинвариантов монодромии, т. е. фактор группы гомологий $\mathscr{H}_{1}\left(X_{y}, \mathbb{Z}_{2}\right)$ по сумме образов гомоморфизмов $F_{\xi}-$ id и $F_{\eta}-$ id. Поскольку $F_{\eta}$ задается матрицей $\left(\begin{array}{ll}1 & 1 \\ 1 & 0\end{array}\right)$ с элементами из $\mathbb{Z}_{2}$, гомоморфизм $F_{\eta}$ - id сюръективен, и группа $\mathrm{H}_{1}\left(Y, \mathscr{H}_{1}\left(X_{y}, \mathbb{Z}_{2}\right)\right)$ тривиальна. Ясно, что классы слоя и сечения выживают в $\mathrm{H}_{2}\left(X, \mathbb{Z}_{2}\right.$ ) (это означает, что спектральная последовательность вырождается в члене $E_{p, q}^{2}$ ), откуда мы получаем, что $\mathrm{H}_{2}\left(X, \mathbb{Z}_{2}\right)=\mathbb{Z}_{2}\left\langle\left[X_{y}\right],\left[\sigma_{0}\right]\right\rangle$.

Теперь укажем два лагранжевых вложения бутылки Клейна в $X$. Рассмотрим следующие кривые в слое $\Sigma$ :

$$
\beta_{0}:=\left\{t e^{2 \pi i / 3} \in \Sigma=\mathbb{C} / \Lambda: t \in \mathbb{R}\right\}, \quad \beta_{1}:=\left\{\frac{1}{2} e^{\pi i / 3}+t e^{2 \pi i / 3} \in \Sigma=\mathbb{C} / \Lambda: t \in \mathbb{R}\right\} .
$$

Обе кривые являются замкнутыми геодезическими на $\Sigma$, представляющими один и тот же гомологический класс, соответствующий $\beta=e^{2 \pi i / 3} \in \Lambda \cong$ $H_{1}(\Sigma, \mathbb{Z})$. Кроме того, обе эти кривые инвариантны относительно преобразования $F_{\xi}=-\mathrm{id}$. В случае кривой $\beta_{1}$ это следует из того, что вектор $2 \cdot \frac{1}{2} e^{\pi i / 3}=e^{\pi i / 3}=e^{2 \pi i / 3}+1$ принадлежит решетке $\Lambda$. 
Рассмотрим вещественные поверхности $K_{0}$ и $K_{1}$ в комплексной поверхности $X$, образованные точками $(y, z) \in X$, первая компонента $y$ которых лежит на кривой $\xi$, а вторая компонента $z$ - на кривой $\beta_{0}$ или $\beta_{1}$ соответственно. Другими словами, мы выбираем точку $y_{0} \in \xi$, реализуем кривые $\beta_{0}$ и $\beta_{1}$ в слое $\operatorname{pr}^{-1}\left(y_{0}\right)$ и обносим их вокруг кривой $\xi$ с помощью параллельного переноса. Поскольку $F_{\xi}\left(\beta_{i}\right)=\beta_{i}$, поверхности $K_{0}$ и $K_{1}$ замкнуты. Из того что преобразование $F_{\xi}$ обращает ориентацию на каждой из кривых $\beta_{i}$, мы получаем, что эти поверхности являются бутылками Клейна. Тот факт, что симплектическая форма $\omega$ локально (по базе) является прямым произведением форм на базе и слое, обеспечивает лагранжевость $K_{i}$ относительно $\omega$.

Для вычисления гомологических классов $\left[K_{i}\right] \in \mathrm{H}_{2}\left(X, \mathbb{Z}_{2}\right)$ заметим следующее. Поскольку поверхности $K_{i}$ не пересекают общий слой расслоения $\mathrm{pr}: X \rightarrow Y$, каждая из них должна быть либо гомологична слою, либо гомологически тривиальна. Поскольку $K_{1}$ не пересекает сечение $\sigma_{0}$, мы получаем $\left[K_{1}\right]=0 \in \mathrm{H}_{2}\left(X, \mathbb{Z}_{2}\right)$. С другой стороны, так как пара $\beta_{0}, \beta_{1}$ разбивает тор $\Sigma$, рассуждение из доказательства предложения 1.7 с заменой меридиана $\boldsymbol{m}$ на объединение $\beta_{0} \cup \beta_{1}$ показывает, что сумма $\left[K_{0}\right]+\left[K_{1}\right]$ гомологична слою. Таким образом, $\left[K_{0}\right]=\left[X_{y}\right] \neq 0 \in \mathrm{H}_{2}\left(X, \mathbb{Z}_{2}\right)$.

\section{§ 2. Комбинаторная структура групп классов отображений}

2.1. Группы Кокстера-Вейля и группы кос. Напомним стандартные определения и факты о группах Кокстера-Вейля и группах кос Артина-Брискорна.

Пусть $\mathcal{S}=\left\{s_{1}, \ldots, s_{r}\right\}$ - конечное множество. Матрицей Кокстера над $\mathcal{S}$ называется симметрическая $(r \times r)$-матрица $M=\left(m_{i j}\right)$ с элементами из $\mathbb{N} \cup\{\infty\}$, у которой $m_{i i}=1$ и $m_{i j} \geqslant 2$ при $i \neq j$. Пара $(\mathcal{S}, M)$ называется системой Кокстера. Зачастую мы будем обозначать систему Кокстера просто $\mathcal{S}$ и считать $M$ структурой на системе $\mathcal{S}$, обозначая ее $M_{\mathcal{S}}$. Для пары элементов $s=s_{i}$, $s^{\prime}=s_{j} \in \mathcal{S}$ будем использовать обозначение $m_{s, s^{\prime}}:=m_{i j}$.

Граф Кокстера $\Delta=\Delta(\mathcal{S}, M)$, ассоциированный с системой $(\mathcal{S}, M)$, имеет множество $\mathcal{S}$ вершин; вершины $s_{i}$ и $s_{j}$ соединены ребром тогда и только тогда, когда $m_{i j} \geqslant 3$; если $m_{i j} \geqslant 4$, то ребру приписывается вес $m_{i j}=m_{j i}$. Взвешенный граф Кокстера $\Delta$ однозначно определяет соответствующую матрицу Кокстера.

Для всякого подмножества $\mathcal{S}^{\prime} \subset \mathcal{S}$ индуцированная матрица Кокстера $M^{\prime}$ есть ограничение $M=M_{\mathcal{S}}$ на $\mathcal{S}^{\prime}$. Ассоциированный граф $\Delta^{\prime}:=\Delta\left(\mathcal{S}^{\prime}, M^{\prime}\right)$ является полным подграфом в $\Delta$, т. е. две вершины $s_{1}, s_{2} \in \mathcal{S}^{\prime}$ соединены в $\Delta^{\prime}$ так же, как и в $\Delta$ (с учетом весов).

Для любых двух букв $a, b$ и неотрицательного целого $m$ обозначим через $\langle a b\rangle^{m}$ слово $a b a b \ldots$ длины $m$, состоящее из чередующихся букв $a$ и $b$. Если $a$ и $b$ лежат в группе $G$, то $\langle a b\rangle^{m}$ обозначает соответствующее произведение в группе $G$.

ОПРЕДЕЛЕНИЕ 2.1. Группа кос Артина-Брискорна $\operatorname{Br}(\mathcal{S})=\operatorname{Br}(\mathcal{S}, M)$ системы Кокстера $(\mathcal{S}, M)$ - это группа, порожденная элементами множества $\mathcal{S}$ 
с (обобщенными) соотношениями группы кос

$$
\left\langle s_{i} s_{j}\right\rangle^{m_{i j}}=\left\langle s_{j} s_{i}\right\rangle^{m_{i j}}
$$

для всех $i \neq j$ таких, что $m_{i j}<\infty$.

Группа Кокстера-Вейля $\mathrm{W}(\mathcal{S})=\mathrm{W}(\mathcal{S}, M)$ получается из группы кос $\operatorname{Br}(\mathcal{S})$ добавлением соотношения идемпотентности $s^{2}=1$ для каждого $s \in \mathcal{S}$.

Ядро естественной проекции $\pi: \operatorname{Br}(\mathcal{S}) \rightarrow \mathrm{W}(\mathcal{S})$ называется группой крашеных кос системы Кокстера $(\mathcal{S}, M)$ и обозначается $\mathrm{P}(\mathcal{S})$. Ее абелианизация обозначается $\mathrm{P}_{\mathrm{ab}}(\mathcal{S})$.

Для того чтобы отличать тождества в различных группах, мы будем использовать запись $w \equiv w^{\prime}$ для равенства в группе $\mathrm{W}(\mathcal{S})$ и $w=w^{\prime}$ для равенства в группе $\operatorname{Br}(\mathcal{S})$. Выражсение элемента $w$ из группы $\mathrm{W}(\mathcal{S})$ или $\operatorname{Br}(\mathcal{S})$ - это слово $s=s_{1}^{\epsilon_{1}} \cdots s_{q}^{\epsilon_{q}}$ в алфавите $\mathcal{S}$ с показателями $\epsilon_{i}= \pm 1$, значение которого в $\mathrm{W}(\mathcal{S})$ или, соответственно, в $\operatorname{Br}(\mathcal{S})$ равно $w$. Такое выражение $s$ называется положительным, если все показатели $\epsilon_{i}$ равны +1 . С учетом соотношения $s_{i}^{-1}=s_{i}$ мы будем рассматривать только положительные выражения в группе $w \in \mathrm{W}(\mathcal{S})$. Элемент $w \in \operatorname{Br}(\mathcal{S})$ называется положителъным, если у него существует положительное выражение.

ОПРЕДЕЛЕНИЕ 2.2. Длиной $\ell(w)$ элемента $w$ из группы $\mathrm{W}(\mathcal{S})$ называется минимально возможная длина выражения $s=s_{1} \cdots s_{l}$ этого элемента. W-длина элемента $x \in \operatorname{Br}(\mathcal{S})$ - это длина $\ell_{\mathrm{W}}(\bar{x})$ его проекции $\bar{x}:=\pi(x)$ в $\mathrm{W}(\mathcal{S})$. Выражение $s$ называется приведенным (или $\mathbf{W}$-приведенным), если оно положительно и его длина равна его W-длине.

Квазиотражением в группе Кокстера-Вейля $\mathrm{W}(\mathcal{S})$ называется элемент $t$, сопряженный какой-то образующей $s \in \mathcal{S}$. Обозначим через $\mathcal{T}(\mathcal{S})$ множество всех квазиотражений в группе $\mathrm{W}(\mathcal{S})$.

Отметим, что квазиотражения являются обычными отражениями в конечных группах Кокстера-Вейля и транспозициями в симметрических группах $\mathrm{Sym}_{n}$. Нам понадобятся следующие стандартные свойства групп КокстераВейля (см., например, [15] или [27]).

ПредлОжЕНИЕ 2.3. 1. Сильное свойство замены. Пусть $s=s_{1} \cdots s_{q} \in$ $\mathrm{W}(\mathcal{S})$ - положстельное выражение элемента $w \in \mathrm{W}(\mathcal{S}), s \in \mathcal{S}$ - образующая, a $t \in \mathcal{T}(\mathcal{S}, M)$ - квазиотражение. Тогда $\ell(w s)=\ell(w) \pm 1, a \ell(w t)-\ell(w)$ нечетно. Кроме того, если $\ell(w t)<\ell(w)$, то найдется индекс $j=1, \ldots, q$ такой, что $w \equiv s_{1} \cdots s_{j-1} s_{j+1} \cdots s_{q} t$. Аналогичные утверждения выполнены и для умножения на элемент $w$ справа.

2. Соотношения между образующими. Порядок элемента $s_{i} s_{j}$ в группе $\mathrm{W}(\mathcal{S})$ равен $m_{i j}$. Далее, для любого подмножества $\mathcal{S}^{\prime} \subset \mathcal{S}$ естественный гомоморфизм $\mathrm{W}\left(\mathcal{S}^{\prime}\right) \rightarrow \mathrm{W}(\mathcal{S})$ ингективен, т.е. в группе $\mathrm{W}(\mathcal{S})$ нет никаких дополнительных соотношений между образующими группь $\mathrm{W}\left(\mathcal{S}^{\prime}\right)$.

3. Единственность множества сомножителей. Для любого элемента $w \in$ $\mathrm{W}(\mathcal{S})$ множество сомножителей $\mathcal{S}_{w}:=\left\{s_{1}, \ldots, s_{l}\right\}$ в приведенном выражении $w=s_{1} \cdots s_{l}$ не зависит от выбора приведенного выражения; таким образом, множество $\mathcal{S}_{w}$ порождает единственную минимальную подсистему $\mathcal{S}^{\prime} \subset \mathcal{S}$, для которой элемент $w$ лежит в группе $\mathrm{W}\left(\mathcal{S}^{\prime}\right)$. 
4. Классы сопряженности образующих. Две образующие $s^{\prime}, s^{\prime \prime} \in \mathcal{S}$ coпряжены тогда и только тогда, когда их можно соединитъ иепочкой $s_{0}=$ $s^{\prime}, s_{1}, \ldots, s_{n-1}, s_{n}=s^{\prime \prime}$, в которой все индексы $m_{s_{i-1}, s_{i}}$ нечетны. В частности, для связного графа $\Delta(\mathcal{S})$ без весов (m.е. такого, что все $m_{i j}$ равнь 2 или 3 ) множество квазиотражений $\mathcal{T}(\mathcal{S})$ состоит из элементов, сопряженных любой заданной образующей $s \in \mathcal{S}$.

Лемма 2.4. Пусть элемент $w \in \mathrm{W}(\mathcal{S})$ имеет два разложения: $w \equiv s_{1} w_{1} \equiv$ $s_{2} w_{2}$, причем $s_{1} \neq s_{2} \in \mathcal{S}, w_{1}, w_{2} \in \mathrm{W}(\mathcal{S})$ u $\ell\left(w_{1}\right)=\ell\left(w_{2}\right)=\ell(w)-1$. Положим $m:=m_{s_{1} s_{2}}$. Тогда элемент $w$ имеет разложение $w \equiv\left\langle s_{1} s_{2}\right\rangle^{m} w_{0} \equiv\left\langle s_{2} s_{1}\right\rangle^{m} w_{0}$ при $\ell\left(w_{0}\right)=\ell(w)-m$. Аналогичное утверждение выполнено и для разложений вида $w \equiv w_{1}^{\prime} s_{1} \equiv w_{2}^{\prime} s_{2}$.

ДокАЗАТЕЛЬСтво. Выберем разложения $w \equiv\left\langle s_{1} s_{2}\right\rangle^{n_{1}} v_{1}$ и $w \equiv\left\langle s_{2} s_{1}\right\rangle^{n_{2}} v_{2}$ так, что $\ell\left(v_{i}\right)=\ell(w)-n_{i}$ и длины $n_{i}$ являются максимально возможными. Тогда $1 \leqslant n_{i} \leqslant m$. В самом деле, если $n_{1}>m$, то элемент $w$ имеет приведенное выражение, начинающееся с $\left\langle s_{1} s_{2}\right\rangle^{m+1}$. Но тогда

$$
\left\langle s_{1} s_{2}\right\rangle^{m+1}=s_{1}\left\langle s_{2} s_{1}\right\rangle^{m} \equiv s_{1}\left\langle s_{1} s_{2}\right\rangle^{m}=s_{1}^{2}\left\langle s_{2} s_{1}\right\rangle^{m-1} \equiv\left\langle s_{2} s_{1}\right\rangle^{m-1}
$$

имеет меньшую $\mathbf{W}$-длину, что противоречит условию $\ell\left(v_{1}\right)=\ell(w)-n_{1}$.

Если хотя бы одно из чисел $n_{1}, n_{2}$ равно $m$, то доказательство очевидно, поэтому предположим, что $n_{1}, n_{2}<m$. Выберем приведенные выражения $\boldsymbol{v}_{i}$ элементов $v_{i}$ так, что $\left\langle s_{1} s_{2}\right\rangle^{n_{1}} \boldsymbol{v}_{1}$ и $\left\langle s_{2} s_{1}\right\rangle^{n_{2}} \boldsymbol{v}_{2}$ являются приведенными выражениями элемента $w$. Тогда, применяя сильное свойство замены и соотношение $\ell\left(s_{2} w\right)=\ell(w)-1$, мы получаем, что элемент $w$ имеет выражение $s_{2} \boldsymbol{w}_{2}$, в котором $\boldsymbol{w}_{2}$ получается удалением одной образующей $s^{*}$ из выражения $\left\langle s_{1} s_{2}\right\rangle^{n_{1}} \boldsymbol{v}_{1}$. Эта образующая не может быть первой буквой $s_{1}$, так как $s_{1} \neq s_{2}$. Она не может быть и последней буквой в подслове $\left\langle s_{1} s_{2}\right\rangle^{n_{1}}$, так как тогда $\left\langle s_{1} s_{2}\right\rangle^{n_{1}} \equiv\left\langle s_{2} s_{1}\right\rangle^{n_{1}}$, что противоречит неравенству $n_{1}<m=m_{s_{1} s_{2}}$. Наконец, она не может быть внутренней буквой в подслове $\left\langle s_{1} s_{2}\right\rangle^{n_{1}}$, поскольку тогда мы получили бы квадрат образующей в качестве подслова в $s_{2} \boldsymbol{w}_{2}$, откуда следовало бы, что $\ell\left(s_{2} \boldsymbol{w}_{2}\right)<\ell(w)$. Итак, буква $s^{*}$ удаляется из слова $\boldsymbol{v}_{1}$. Обозначив полученное таким образом слово через $\boldsymbol{v}_{2}^{\prime}$, мы имеем приведенное выражение $\left\langle s_{2} s_{1}\right\rangle^{n_{1}+1} \boldsymbol{v}_{2}^{\prime}$. Отсюда следует, что $n_{2}>n_{1}$. В силу симметрии должно быть выполнено и обратное неравенство $n_{1}>n_{2}$. Полученное противоречие показывает, что на самом деле $n_{1}=n_{2}=m$, что и требовалось доказать.

ЛЕмма 2.5. Для любых двух приведенных выражений $\boldsymbol{s}=s_{1} \cdots s_{l} u \boldsymbol{s}^{\prime}=$ $s_{1}^{\prime} \cdots s_{l}^{\prime}$ элемента $w \in \mathrm{W}(\mathcal{S})$ их значения в группе $\operatorname{Br}(\mathcal{S})$ определяют один и тот же элемент $\widehat{w} \in \operatorname{Br}(\mathcal{S})$. В частности, соответствие $w \in \mathrm{W}(\mathcal{S}) \mapsto \widehat{w} \in$ $\operatorname{Br}(\mathcal{S})$ является теоретико-множественным сечением проекции $\pi: \operatorname{Br}(\mathcal{S}) \rightarrow$ $\mathrm{W}(\mathcal{S})$.

ДоКАЗАТЕЛЬСтво. Используя индукцию по длине элемента $w$, мы можем считать, что утверждение справедливо для всех элементов $v \in \mathrm{W}(\mathcal{S})$ длины $\ell(v)<\ell(w)$. Отсюда немедленно следует утверждение леммы при $s_{1}=s_{1}^{\prime}$, поэтому рассмотрим случай $s_{1} \neq s_{1}^{\prime}$. Положим $m:=m_{s_{1}, s_{1}^{\prime}}$. По лемме 2.4 элемент $w$ имеет приведенные выражения $\left\langle s_{1} s_{1}^{\prime}\right\rangle^{m} w_{0} \equiv\left\langle s_{1}^{\prime} s_{1}\right\rangle^{m} w_{0}$. По предположению индукции подъем $\widehat{w}_{0} \in \operatorname{Br}(\mathcal{S})$ корректно определен. Поскольку 
$\left\langle s_{1} s_{1}^{\prime}\right\rangle^{m}=\left\langle s_{1}^{\prime} s_{1}\right\rangle^{m}$ в группе $\operatorname{Br}(\mathcal{S})$, последние два разложения определяют один и тот же элемент $\left\langle s_{1} s_{1}^{\prime}\right\rangle^{m} \widehat{w}_{0}=\left\langle s_{1}^{\prime} s_{1}\right\rangle^{m} \widehat{w}_{0} \in \operatorname{Br}(\mathcal{S})$. С другой стороны, элемент $\hat{s}:=s_{1} \cdots s_{l} \in \operatorname{Br}(\mathcal{S})$ равен элементу $\left\langle s_{1} s_{1}^{\prime}\right\rangle^{m} \widehat{w}_{0}$ в группе $\operatorname{Br}(\mathcal{S})$, поскольку они имеют одинаковые первые буквы $s_{1}$. Повторяя это рассуждение для $\hat{s}^{\prime}:=s_{1}^{\prime} \cdots s_{l}^{\prime}$, мы завершаем доказательство леммы.

Применяя теорему Райдемайстера-Шрайера (см., например, [16] или [34]), мы получаем копредставление группы $\mathrm{P}(\mathcal{S})$.

ПРЕДЛОЖЕНИЕ 2.6. і) Чистая группа кос $\mathrm{P}(\mathcal{S})$ порождается элементами $\left(s_{1} \cdots s_{l}\right) \cdot s_{0}^{2} \cdot\left(s_{1} \cdots s_{l}\right)^{-1}$, для которых произведение $s_{1} \cdots s_{l} s_{0}$ является W-приведенным.

ii) Определяющее множество соотношений Райдемайстера-Шрайера $в$ группе $\mathrm{P}(\mathcal{S})$ coстоит из произведениц $\widehat{w} \cdot R \cdot \widehat{w}^{-1}$, в которых $w$ пробегает группу Кокстера-Вейля $\mathrm{W}(\mathcal{S}), \quad R$ пробегает определяющее множество соотношений в группе кос $\operatorname{Br}(\mathcal{S})$, a $\widehat{w} \cdot R \cdot \widehat{w}^{-1}$ записывается как произведение указанных выше образующих $\left(s_{1} \cdots s_{l}\right) \cdot s_{0}^{2} \cdot\left(s_{1} \cdots s_{l}\right)^{-1}$.

ОПРЕДЕЛЕНИЕ 2.7. Полученные таким образом элементы называются образующими и соотношениями Райдемайстера-Шрайера, или просто RS-элементами. Кроме того, если обозначить через $a * b$ сопряжение элемента $b$ элементом $a$, так что $a * b:=a b a^{-1}$, то все RS-образующие имеют вид $\widehat{w} * s_{0}^{2}$.

Полученные с помощью теоремы Райдемайстера-Шрайера образующие являются элементами свободной группы $\operatorname{Fr}(\mathcal{S})$, и их явный вид зависит от конкретного выражения $s_{1} \cdots s_{l}$. Однако в силу леммы 2.5 нам достаточно знать соответствующий элемент в группе $\mathrm{W}(\mathcal{S})$.

ДОКАЗАТЕЛЬСТВО ПРЕДЛОЖЕНИЯ 2.6. МЫ ограничимся указанием алгоритма, представляющего всякий элемент $f \in \mathrm{P}(\mathcal{S})$ в виде произведения образующих. Пусть $f=f_{1} \cdot \widehat{w} \cdot s^{\epsilon} \cdot f_{2}$, где $f_{1}-$ произведение RS-образующих, $\widehat{w}-$ $\mathrm{W}$-приведенный элемент в $\operatorname{Br}(\mathcal{S}), s^{\epsilon}=s^{ \pm 1}$ - буква с $s \in \mathcal{S}$, а $f_{2}$ - произвольный элемент группы $\operatorname{Br}(\mathcal{S})$. Найдем такой элемент $w_{1} \in \mathrm{W}(\mathcal{S})$, что $w_{1} \equiv w \cdot s$. Тогда $f=f_{1} \cdot\left(\widehat{w} \cdot s^{\epsilon} \cdot \widehat{w}_{1}^{-1}\right) \cdot \widehat{w}_{1} \cdot f_{2}$. Значит, нам достаточно представить элемент $\widehat{w} \cdot s^{\epsilon} \cdot \widehat{w}_{1}^{-1}$ в виде произведения RS-образующих. Необходимо рассмотреть четыре случая в зависимости от возможной $\mathbf{W}$-длины элемента $w_{1}$ и знака $\epsilon= \pm 1$. Напомним, что $\ell_{\mathrm{W}}\left(w_{1}\right)=\ell_{\mathrm{W}}(w) \pm 1$. Имеем:

1) случай $\ell\left(w_{1}\right)=\ell(w)+1$ и $\epsilon=+1: \widehat{w}_{1}=\widehat{w} \cdot s$ и $\widehat{w} \cdot s \cdot \widehat{w}_{1}^{-1}=1$;

2) случай $\ell\left(w_{1}\right)=\ell(w)+1$ и $\epsilon=-1: \widehat{w}_{1}=\widehat{w} \cdot s$ и $\widehat{w} \cdot s^{-1} \cdot \widehat{w}_{1}^{-1}=\left(\widehat{w} * s^{2}\right)^{-1}$;

3) случай $\ell\left(w_{1}\right)=\ell(w)-1$ и $\epsilon=+1: \widehat{w}=\widehat{w}_{1} \cdot s$ и $\widehat{w} \cdot s \cdot \widehat{w}_{1}^{-1}=\widehat{w}_{1} * s^{2}$;

4) случай $\ell\left(w_{1}\right)=\ell(w)-1$ и $\epsilon=-1: \widehat{w}=\widehat{w}_{1} \cdot s$ и $\widehat{w} \cdot s^{-1} \cdot \widehat{w}_{1}^{-1}=1$.

Предложение доказано.

ПРеДЛОЖЕНИЕ 2.8. і) Абелианизация $\mathrm{P}_{\mathrm{ab}}(\mathcal{S})$ группъ крашеных кос $\mathrm{P}(\mathcal{S})$ является свободной абелевой группой с базисом, порожденным RS-элементами $\widehat{w} * s^{2} \in \mathrm{P}(\mathcal{S})$.

ii) Проекция элемента $x * s^{2} n p u x \in \operatorname{Br}(\mathcal{S})$ u $s \in \mathcal{S}$ в группу $\mathrm{P}_{\mathrm{ab}}(\mathcal{S})$ является образующей вида $\left[\widehat{w} * \tilde{s}^{2}\right]_{\mathrm{ab}}$ для некоторых $w \in \mathrm{W}(\mathcal{S})$ u $\tilde{s} \in \mathcal{S}$, вообще говоря, не единственных. Кроме того, элемент $\widehat{w} * \tilde{s}$ может быть получен применением последовательности следующих преобразований: 
$(\mathrm{A} 0)$ замена $x * s^{2}$ при $x \in \operatorname{Br}(\mathcal{S})$ на $x^{\prime} * s^{2}$ для такого $x^{\prime} \in \operatorname{Br}(\mathcal{S})$, что $x$ и $x^{\prime}$ имеют одинаковые проекиии в группу $\mathrm{W}(\mathcal{S}), x \equiv x^{\prime} \in \mathrm{W}(\mathcal{S})$;

(A1) замена $\left(x \cdot\left\langle s_{1} s_{2}\right\rangle^{k}\right) * s_{3}^{2}$ на $\left(x \cdot\left\langle s_{2} s_{1}\right\rangle^{m-k-1}\right) *\left(s_{3}^{\prime}\right)^{2}$, где:

a) $x \in \operatorname{Br}(\mathcal{S}), s_{1}, s_{2} \in \mathcal{S}, m:=m_{s_{1} s_{2}}<\infty, k=0, \ldots, m-1$,

b) $s_{3}=s_{1}$, если $k$ четно, и $s_{3}=s_{2}$, если $k$ нечетно,

c) $s_{3}^{\prime}=s_{3}$, если $m$ четно, и $s_{3}^{\prime}$ - недостающий элемент $s_{1}$ или $s_{2}$, если $m$ нечетно.

ДокАзАТЕЛЬСтво. Покажем сначала, что преобразования (А0) и (A1) тождественны в группе $\mathrm{P}_{\mathrm{ab}}(\mathcal{S})$. Первое следует из равенства

$$
\left(x s_{1}^{2} y\right) * s_{2}^{2}=\left(x s_{1}^{2} y\right) s_{2}^{2}\left(x s_{1}^{2} y\right)^{-1}=x s_{1}^{2} x^{-1} \cdot(x y) s_{2}^{2}(x y)^{-1} \cdot\left(x s_{1}^{2} x^{-1}\right)^{-1} .
$$

Для доказательства второго заметим, что достаточно рассмотреть случай $x=1$. Представим соотношение группы кос $\left\langle s_{1} s_{2}\right\rangle^{m}=\left\langle s_{2} s_{1}\right\rangle^{m}$ в виде $\left\langle s_{1} s_{2}\right\rangle^{m-1} s_{3}=s_{2}\left\langle s_{1} s_{2}\right\rangle^{m-1}$. Отсюда алгебраическими преобразованиями мы получаем соотношение

$$
\left\langle s_{1} s_{2}\right\rangle^{m-1} s_{3}\left(\left\langle s_{1} s_{2}\right\rangle^{m-1}\right)^{-1}=s_{2},
$$

из которого возведением в квадрат получается требуемое соотношение при $k=$ $m-1$. Сопряжение элементом $\left(\left\langle s_{1} s_{2}\right\rangle^{l}\right)^{-1}$ и применение преобразования (А0) завершают доказательство. В специальном случае $s_{1}=s_{2}=: s$ должно быть выполнено $m=1$ и $k=0$, так что $m-k-1=0$, и единственное соотношение вида (А1) - это тривиальное тождество $(x \cdot s) * s^{2}=x * s^{2}$.

Выберем элемент $a \neq b \in \mathcal{S}$ так, что $m:=m_{a b} \neq \infty$. Пусть $w_{0} \in \mathrm{W}(\mathcal{S})-$ произвольный элемент. Положим $a_{2 i-1}:=b_{2 i}:=a, a_{2 i}:=b_{2 i-1}:=b, w_{i}:=$ $w_{i} \cdot\langle a b\rangle^{i}$ и $w_{i}^{\prime}:=w_{i} \cdot\langle b a\rangle^{i}$. Заметим, что $\langle a b\rangle^{2 m} \equiv 1 \in \mathrm{W}(\mathcal{S})$, так что элементы $w_{i}$ и $w_{i}^{\prime}$ повторяются с периодом $2 m$, т. е. $w_{i+2 m} \equiv w_{i}$ и $w_{i+2 m}^{\prime} \equiv w_{i}^{\prime}$. Кроме того, $b_{i}=a_{2 m-i}$ и $w_{i}^{\prime} \equiv w_{2 m-i}$. Индуцированное RS-соотношение имеет вид

$$
\prod_{i=1}^{m}\left(\widehat{w}_{i-1} \cdot a_{i} \cdot \widehat{w}_{i}^{-1}\right)=\prod_{i=1}^{m}\left(\widehat{w}_{i-1}^{\prime} \cdot b_{i} \cdot\left(\widehat{w}_{i}^{\prime}\right)^{-1}\right) .
$$

Пусть $w_{k}$ - элемент наибольшей $\mathbf{W}$-длины. Тогда $\ell\left(w_{k-1}\right)=\ell\left(w_{k+1}\right)=$ $\ell\left(w_{k}\right)-1$. В этой ситуации лемма 2.4 гарантирует, что $v:=w_{k \pm m}-$ самый короткий элемент для $\ell(v)=\ell\left(w_{k}\right)-m$, и для любого $i=0, \ldots, 2 m$ выполнено $w_{k \pm m \pm i} \equiv v\langle a b\rangle^{i}$ или $w_{k \pm m \pm i} \equiv v\langle b a\rangle^{i}$. Следовательно, $w_{i} \equiv v \cdot v_{i}$, где $\ell\left(w_{i}\right)=\ell(v)+\ell\left(v_{i}\right)$, и $v_{0}$ есть либо $\langle a b\rangle^{l}$, либо $\langle b a\rangle^{l}$ для какого-то $l=0, \ldots, m$. Это означает, что рассматриваемое нами соотношение получается сопряжением элементом $v$ из соотношения

$$
\prod_{i=1}^{m}\left(\hat{v}_{i-1} \cdot a_{i} \cdot \hat{v}_{i}^{-1}\right)=\prod_{i=1}^{m}\left(\hat{v}_{i-1}^{\prime} \cdot b_{i} \cdot\left(\hat{v}_{i}^{\prime}\right)^{-1}\right),
$$

в котором $v_{i}$ и $v_{i}^{\prime}$ определяются так же, как $w_{i}$ и $w_{i}^{\prime}$. 
Учитывая симметрию $a \leftrightarrow b$, предположим, что $v_{0}=\langle a b\rangle^{l}$ при $l \leqslant m$. В случае четного $l$ мы получаем следующую последовательность слов $v_{i}$ и $v_{i}^{\prime}$ :

$$
v_{i} \equiv\left\{\begin{array} { l l } 
{ \langle a b \rangle ^ { l + i } , } & { i = 0 , \ldots , m - l , } \\
{ \langle a b \rangle ^ { m } \equiv \langle b a \rangle ^ { m } , } & { i = m - l , } \\
{ \langle b a \rangle ^ { m + l - i } , } & { i = m - l , \ldots , m , }
\end{array} v _ { i } ^ { \prime } \equiv \left\{\begin{array}{ll}
\langle a b\rangle^{l-i}, & i=0, \ldots, l, \\
\langle a b\rangle^{0} \equiv\langle b a\rangle^{0} \equiv 1, & i=m-l, \\
\langle b a\rangle^{i-l}, & i=l, \ldots, m .
\end{array}\right.\right.
$$

В случае нечетного $l$ эти последовательности меняются местами. Элемент $\hat{v}_{i-1} \cdot a_{i} \cdot \hat{v}_{i}^{-1}$ нетривиален тогда и только тогда, когда $\ell\left(v_{i-1}\right)>\ell\left(v_{i}\right)$; аналогичное утверждение верно и для элемента $\hat{v}_{i-1}^{\prime} \cdot b_{i} \cdot\left(\hat{v}_{i}^{\prime}\right)^{-1}$. Соответствующие значения $i$ суть $i=m-l+1, \ldots, m$ для $v_{i}$ и $i=1, \ldots, l$ для $v_{i}^{\prime}$. Сравнивая соответствующие сомножители, мы видим, что индуцированное соотношение в группе $\mathrm{P}_{\mathrm{ab}}(\mathcal{S})$ есть сумма соотношений вида $(\mathrm{A} 1)$ по всем $k=1, \ldots, l$.

Tеорема 2.9. Два квазиотражения $t=w * s u t^{\prime}=w^{\prime} * s^{\prime}$ в групnе $\mathrm{W}(\mathcal{S})$ для $w, w^{\prime} \in \mathrm{W}(\mathcal{S})$ u $s, s^{\prime} \in \mathcal{S}$ равны тогда и только тогда, когда второе может быть получено из первого последовательностъю преобразований следующего вида:

(T) замена $\left(x \cdot\left\langle s_{1} s_{2}\right\rangle^{k}\right) * s_{3}$ на $\left(x \cdot\left\langle s_{2} s_{1}\right\rangle^{m-k-1}\right) * s_{3}^{\prime}$, где $x \in \mathrm{W}(\mathcal{S})$, а обозначения $s_{1}, s_{2}, s_{3}, s_{3}^{\prime}$ имеют тот же смысл, что и в части (A1) предложения 2.8.

$B$ частности, сопоставление $t \equiv w * s \in \mathcal{T}(\mathcal{S}) \leftrightarrow A_{t}:=\widehat{w} * s^{2} \in \mathrm{P}_{\mathrm{ab}}(\mathcal{S})$ для $w \in \mathrm{W}(\mathcal{S})$ и $s \in \mathcal{S}$ задает биективное соответствие между множеством квазиотражений в группе $\mathrm{W}(\mathcal{S})$ и $\mathrm{RS}$-базисом группь $\mathrm{P}_{\mathrm{ab}}(\mathcal{S})$.

ДоказАтеЛьСтво. Обозначим через $\mathcal{A}(\mathcal{S})$ построенный выше RS-базис группы $\mathrm{P}_{\mathrm{ab}}(\Delta)$ и через $\left[\widehat{w} * s^{2}\right]_{\text {аь }}$ образ элемента $\widehat{w} * s^{2}=\widehat{w} \cdot s^{2} \cdot \widehat{w}^{-1}$ в группе $\mathcal{A}(\mathcal{S})$. Заметим, что преобразование $(\mathrm{A} 1)$ из предложения 2.8 получается возведением преобразования (Т) в квадрат, а аналогичное преобразованию (A0) преобразование в $\mathcal{T}(\mathcal{S})$ тождественно. Таким образом, второе утверждение теоремы следует из первого.

В любом случае мы получаем корректно определенное сюръективное отображение $\boldsymbol{t}: \mathcal{A}(\mathcal{S}) \rightarrow \mathcal{T}(\mathcal{S})$, задаваемое формулой $\boldsymbol{t}:\left[\widehat{w} * s^{2}\right]_{\text {аb }} \mapsto w * s$, и первое утверждение теоремы эквивалентно инъективности отображения $\boldsymbol{t}$. Поскольку отображение $\boldsymbol{t}$ заведомо $\mathrm{W}(\mathcal{S})$-инвариантно, достаточно доказать, что для любых $s \in \mathcal{S}$ и $w \in \mathrm{W}(\mathcal{S})$ из условия $w * s \equiv s$ вытекает равенство $\left[\widehat{w} * s^{2}\right]_{\mathrm{ab}}=\left[s^{2}\right]_{\mathrm{ab}}$. Проведем индукцию по длине $\ell(w)$. Случай $\ell(w)=0$ тривиален. Зафиксируем пару $(w, s)$, для которой $w * s \equiv s$. Пусть $\ell(w s)=\ell(w)-1$. Тогда по сильному свойству замены имеем $w=w^{\prime} s$, где $\ell\left(w^{\prime}\right)=\ell(w)-1$. Но тогда

$$
w * s \equiv\left(w^{\prime} s\right) * s \equiv\left(w^{\prime} s\right) \cdot s \cdot\left(s\left(w^{\prime}\right)^{-1}\right) \equiv w^{\prime} \cdot s \cdot\left(w^{\prime}\right)^{-1} \equiv w^{\prime} * s,
$$

следовательно, $\left[\widehat{w} * s^{2}\right]_{\mathrm{ab}}=\left[\widehat{w}^{\prime} * s^{2}\right]_{\mathrm{ab}}=\left[s^{2}\right]_{\mathrm{ab}}$ по предположению индукции. Если $\ell(w s)=\ell(w)+1$, то из леммы 2.5 следует равенство $\widehat{w s}=\widehat{w s}$ в группе $\operatorname{Br}(\mathcal{S})$. С другой стороны, равенство $w * s \equiv s$ означает, что элементы $w$ и $s$ коммутируют в группе $\mathrm{W}(\mathcal{S})$, и потому $\ell(s w)=\ell(w s)=\ell(w)+1$. Как и выше, мы получаем, что $\widehat{s w}=s \widehat{w}$, следовательно, $\widehat{w} s=s \widehat{w}$. Отсюда вытекает нужное равенство $\left[\widehat{w} * s^{2}\right]_{\mathrm{ab}}=\left[s^{2}\right]_{\mathrm{ab}}$. Теорема доказана. 
Заметим, что естественный подъем $w \in \mathrm{W}(\mathcal{S}) \mapsto \widehat{w} \in \operatorname{Br}(\mathcal{S})$, построенный в лемме 2.5, не совместим с сопряжением и, в частности, подъем $\hat{t}$ квазиотражения $t$ не сопряжен никакой образующей $s \in \mathcal{S}$, если только само $t$ не является образующей. Для приложений желательно найти естественное поднятие $t \in \mathcal{T}(\mathcal{S}) \mapsto \tilde{t} \in \operatorname{Br}(\mathcal{S})$, для которого всякий элемент $\tilde{t}$ имеет вид $\tilde{t}=x * s$ и проектируется в элемент $t$ группы $\mathrm{W}(\mathcal{S})$. В этом случае множество $\left\{\tilde{t}^{2}: t \in \mathcal{T}(\mathcal{S})\right\}$ будет естественной системой образующих группы $\mathrm{P}(\mathcal{S})$. Наивное определение такого поднятия состоит в том, чтобы найти выражение вида $t \equiv w * s \in \mathrm{W}(\mathcal{S})$ при $s \in \mathcal{S}$ и $w \in \mathrm{W}(\mathcal{S})$ и положить $\tilde{t}:=\widehat{w} * s \in \operatorname{Br}(\mathcal{S})$. Оказывается, однако, что это определение некорректно. Например, в симметрической группе $\mathrm{Sym}_{3}$ со стандартными образующими $a, b$, удовлетворяющими стандартному соотношению $a b a=b a b$, квазиотражение $t:=a b a \equiv b a b \in$ Sym $_{3}$ имеет два различных естественных поднятия в соответствующую группу кос $\mathrm{Br}_{3}: a b a^{-1}=b^{-1} a b$ и $b a b^{-1}=a^{-1} b a$.

Тем не менее, ситуация не так уж плоха, и поднятие $t \in \mathcal{T}(\mathcal{S}) \mapsto \tilde{t} \in \operatorname{Br}(\mathcal{S})$, обладающее вышеуказанными свойствами, существует, хотя оно и не единственно. Для его описания нам понадобится следующее определение.

ОПРЕДЕЛЕНИЕ 2.10. Определим ширину $\operatorname{wd}(t)$ квазиотражения $t \in \mathcal{T}(\mathcal{S})$ как наименьшее такое $k$, что квазиотражение $t$ может быть представлено в виде $t \equiv w * s$, где $w \in \mathrm{W}(\mathcal{S}), s \in \mathcal{S}$ и $k=\ell(w)$. Квазиотражения ширины 0 суть в точности образующие $s \in \mathcal{S}$.

ТеОрема 2.11. і) Eсли $\ell(s t s)=\ell(t)$ для квазиотражения $t \in \mathcal{T}(\mathcal{S})$ и образующей $s \in \mathcal{S}$, то они коммутируют в $\mathrm{W}(\mathcal{S})$, m. e. sts $\equiv t$.

ii) Для любого квазиотражения $t \in \mathcal{T}(\mathcal{S})$ выполнено $\ell(t)=2 \mathrm{wd}(t)+1$. Кроме того, если $\ell(s t)=\ell(t)-1$ для образующей $s \in \mathcal{S}$, то либо $t=s u \ell(t)=1$, либо $\ell(t)>1 u t=s t^{*} s$ для некоторого другого квазиотражения $t^{*} \in \mathcal{T}(\mathcal{S})$ длинь $\ell\left(t^{*}\right)=\ell(t)-2$ и иирины $\mathrm{wd}\left(t^{*}\right)=\mathrm{wd}(t)-1$.

iii) Пусть $t \in \mathcal{T}(\mathcal{S})$ - квазиотражсение, а $s \neq s^{\prime} \in \mathcal{S}$ - две образующие, для которых $\ell(s t)=\ell\left(s^{\prime} t\right)=\ell(t)-1$. Положим $m:=m_{s, s^{\prime}}$. Тогда либо $t \equiv\left\langle s s^{\prime}\right\rangle^{m} \equiv\left\langle s^{\prime} s\right\rangle^{m}$ и $m$ нечетно, либо $t \equiv\left\langle s s^{\prime}\right\rangle^{m} * t^{*}$ для некоторого квазиотражения ииринь $\operatorname{wd}\left(t^{*}\right)=\operatorname{wd}(t)-m$.

iv) Пусть $t \in \mathcal{T}(\mathcal{S})$ - квазиотражение ширинъ $k$, a $t \equiv w * s u t \equiv w^{\prime} *$ $s^{\prime}$ - два представляющих $t$ выражения, где $s, s^{\prime} \in \mathcal{S} u w, w^{\prime} \in \mathrm{W}(\mathcal{S})$, причем $\ell(w)=\ell\left(w^{\prime}\right)=k$. Тогда значение выражения $\widehat{w} * s$ в группе $\operatorname{Br}(\mathcal{S})$ может быть получено из значения выражения $\widehat{w}^{\prime} * s^{\prime} \in \operatorname{Br}(\mathcal{S})$ сопряљением элементами вида $t_{i}^{2}=\widehat{w}_{i} * s_{i}^{2} \in \operatorname{Br}(\mathcal{S})$, где $s_{i} \in \mathcal{S}$, a $w_{i} \in \mathrm{W}(\mathcal{S})$, причем $\ell\left(w_{i}\right)<k$.

v) Для каждого $t \in \mathcal{T}(\mathcal{S})$ зафиксируем выражение $t=w_{t} * s_{t}$ при $s_{t} \in \mathcal{S} u$ $w_{t} \in \mathrm{W}(\mathcal{S})$, удовлетворяющее условию $\ell\left(w_{t}\right)=\operatorname{wd}(t)$, и положим $\tilde{t}:=\widehat{w}_{t} * s_{t} \in$ $\operatorname{Br}(\mathcal{S})$. Тогда множество $\widetilde{\mathcal{T}}^{2}:=\left\{\tilde{t}^{2}: t \in \mathcal{T}(\mathcal{S})\right\}$ является минимальным множеством образующих для группы крашеных кос $\mathrm{P}(\mathcal{S})$.

Главным в теореме 2.11 является утверждение iv), которое имеет следующий смысл: подъем $t \in \mathcal{T}(\mathcal{S}) \mapsto \tilde{t}=\widehat{w} * s$, определяемый в утверждении v), единствен по модулю сопряжений на подъемы $\tilde{t}_{i}$ квазиотражений $t_{i}$ меньшей ширины, чем $t, \operatorname{wd}\left(t_{i}\right)<\operatorname{wd}(t)$. 
ДокАЗАТЕЛЬСТво тЕОРЕмЫ 2.11. і) Зафиксируем приведенное выражение $\boldsymbol{t}$ для элемента $t$. Заметим, что $\ell(s t)=\ell\left((s t)^{-1}\right)=\ell\left(t^{-1} s^{-1}\right)=\ell(t s)$. Предположим, что $\ell(t s)=\ell(s t)=\ell(t)+1$. Тогда $\ell(s(t s))=\ell(t s)-1$, и мы можем применить сильное свойство замены к выражению $s \cdot(\boldsymbol{t} \cdot s)$. Первая буква $s$ не может сократиться с буквой из $t$, поскольку тогда $\ell(s t)=\ell(t)-1=\ell(t s)$, что противоречит сделанному выше предположению. Значит, в выражении $s \cdot(\boldsymbol{t} \cdot s)$ первая буква $s$ сокращается с последней, и мы получаем требуемое утверждение. В случае $\ell(t s)=\ell(s t)=\ell(t)-1$ положим $x:=t \cdot s \in \mathrm{W}(\mathcal{S})$, зафиксируем приведенное выражение $\boldsymbol{x}$ для $x$ и применим то же самое рассуждение к произведению $s \cdot(\boldsymbol{x} \cdot s)$. Заметим, что хотя во втором случае утверждение вытекает также из утверждения ii), данное нами доказательство не опирается на iі).

ii) Рассмотрим несколько случаев и подслучаев. Поскольку все вычисления производятся в группе Кокстера $\mathrm{W}(\mathcal{S})$, мы будем использовать для равенства в этой группе символ "=".

Проведем индукцию по ширине $\operatorname{wd}(t)$ квазиотражения. Заметим, что для любого элемента $t \in \mathcal{T}(\mathcal{S})$ его длина $\ell(t)$ нечетна и $\ell(t) \leqslant 2 \mathrm{wd}(t)+1$.

Очевидно, что образующие $s \in \mathcal{S}$ - это единственные квазиотражения ширины 0 , а их длина равна 1 . Это доказывает базу индукции, и нам остается обосновать шаг индукции. Кроме того, если $\operatorname{wd}(t)=1$, то $t=s_{1} s_{2} s_{1}$ и длина $\ell(t)$ равна либо 3 , либо 1 . Во втором случае элементы $s_{1}$ и $s_{2}$ коммутируют, так что $t=s_{1}$, что противоречит условию $\mathrm{wd}(t)=1$. Значит, длина $\ell(t)$ должна быть равна 3. Далее, если $t=s_{1} s_{2} s_{1}$ и $\ell(s \cdot t)=2$, то по сильному свойству замены произведение $s \cdot t$ задается словом, получающимся из $s_{1} s_{2} s_{1}$ удалением одной буквы. В частности, $s$ лежит в подгруппе, порожденной $s_{1}, s_{2}$. Из соотношений между образующими (см. предложение 2.3) следует, что $s$ есть либо $s_{1}$, либо $s_{2}$. В случае $s=s_{1}$ доказтельство очевидно, так что мы можем считать, что $s=s_{2}$. Рассматривая всевозможные слова, получающиеся из $s_{1} s_{2} s_{1}$ удалением одной буквы, мы видим, что единственное такое слово, являющееся выражением для $s \cdot t=s_{2} s_{1} s_{2} s_{1}$, - это $s_{1} s_{2}$. В данном случае мы немедленно получаем, что $s_{1}, s_{2}$ удовлетворяют обычному соотношению $s_{1} s_{2} s_{1}=s_{2} s_{1} s_{2}$, следовательно, $t=s_{2} s_{1} s_{2}=s s_{1} s$, что и требовалось доказать. Итак, оба утверждения части іi) справедливы при $k=1$.

Зафиксируем теперь $k \geqslant 2$. Предположим, что $\ell\left(t_{0}\right)=2 \mathrm{wd}\left(t_{0}\right)+1$ для всех $t_{0} \in \mathcal{T}(\mathcal{S})$ при $\operatorname{wd}\left(t_{0}\right) \leqslant k$ и второе утверждение части iі) выполняется для всех $t_{0} \in \mathcal{T}(\mathcal{S})$ при $\operatorname{wd}\left(t_{0}\right) \leqslant k-1$. Пусть $t$ - квазиотражение ширины $k$, и пусть $s \in \mathcal{S}$ - образующая. Если $\ell(s t s)=\ell(t)+2$, то доказательство очевидно. Поэтому можно считать, что $\ell(s t s) \leqslant \ell(t)$. Тогда в случае $\ell(s t)=\ell(t)+1$ должно выполняться равенство $\ell(s t s)=\ell(t)$. Значит, sts $=t$ в силу части i), так что требуемое соотношение $\ell(t)=2 \mathrm{wd}(t)+1$ выполняется в силу индуктивного предположения. Поэтому мы можем полагать, что $\ell(s t)=\ell(t)-1$.

По условию имеем $t=s^{\prime} t^{\prime} s^{\prime}$ для $s^{\prime} \in \mathcal{S}$ и $t^{\prime} \in \mathcal{T}(\mathcal{S})$ ширины $\operatorname{wd}\left(t^{\prime}\right)=k-1$. Если $s=s^{\prime}$, то доказательство очевидно, поэтому предположим, что $s$ и $s^{\prime}$ различны. Тогда $\ell\left(s^{\prime} t\right)=\ell(t)-1$ и в силу леммы 2.4 мы получаем, что $t=$ $\left\langle s s^{\prime}\right\rangle^{m} x$, где $m=m_{s, s^{\prime}}$ и $x \in \mathrm{W}(\mathcal{S})$ имеет длину $\ell(x)=\ell(t)-m$. Зафиксируем приведенное выражение $\boldsymbol{x}$, представляющее элемент $x$. 
Тривиальный случай: $x=1$, т. е. $t=\left\langle s s^{\prime}\right\rangle^{m}$. В этом случае $m$ нечетно, $m \geqslant 3$, следовательно, $s$ является первой и последней буквой в слове $\left\langle s s^{\prime}\right\rangle^{m}$. Таким образом, sts $=\left\langle s^{\prime} s\right\rangle^{m-2}$, и требуемое утверждение выполнено.

Случай $(\mathrm{a}): \ell(x s)=\ell\left(x s^{\prime}\right)=\ell(x)-1$. Тогда $x=y \cdot\left\langle s s^{\prime}\right\rangle^{m}=y \cdot\left\langle s^{\prime} s\right\rangle^{m}$ для некоторого $y \in \mathrm{W}(\mathcal{S})$ длины $\ell(y)=\ell(x)-m$, и потому $t=\left\langle s^{\prime} s\right\rangle^{m} * y$ и $t^{\prime}=\left\langle s s^{\prime}\right\rangle^{m-1} * y$. В частности, $y$ является квазиотражением, $y \in \mathcal{T}(\mathcal{S})$. Заметим, что к $t^{\prime}$ применимо предположение индукции. Сравнивая длины элементов $t^{\prime}$ и $y$, мы видим, что $\operatorname{wd}(y)=\operatorname{wd}\left(t^{\prime}\right)-(m-1)=\mathrm{wd}(t)-m$. Отсюда следует, что квазиотражение $t^{*}:=\left\langle s^{\prime} s\right\rangle^{m-1} * y$ имеет ширину $\operatorname{wd}\left(t^{*}\right)=\operatorname{wd}(t)-1$ и $t=s t^{*} s$, что и требовалось доказать.

Случай $(\mathrm{b}): \ell(x s)=\ell\left(x s^{\prime}\right)=\ell(x)+1$. Из того что $t s=(s t)^{-1}$, следует, что $\ell(t s)=\ell(t)-1$ и, аналогичным образом, $\ell\left(t s^{\prime}\right)=\ell(t)-1$. Значит, мы можем применить сильное свойство замены (см. предложение 2.3) к произведениям $\left\langle s s^{\prime}\right\rangle^{m} \boldsymbol{x} \cdot s$ и $\left\langle s s^{\prime}\right\rangle^{m} \boldsymbol{x} \cdot s^{\prime}$. В этих двух случаях значение произведения дается словом, получающимся удалением одной буквы из произведения. Поскольку $\ell(x s)=\ell\left(x s^{\prime}\right)=\ell(x)+1$, в двух случаях буква удаляется из подслова $\left\langle s s^{\prime}\right\rangle^{m}$. Отсюда следуют коммутационные тождества $x s=s^{*} x$ и $x s^{\prime}=s^{+} x$, где $s^{*}, s^{+}$ суть $s$ или $s^{\prime}$. Для того чтобы определить, в каком случае $s^{*}=s, s^{+}=s^{\prime}$, а в каком, наоборот, $s^{*}=s^{\prime}, s^{+}=s$, заметим, что $t^{\prime}=s^{\prime} t s^{\prime}=\left\langle s s^{\prime}\right\rangle^{m-1} \boldsymbol{x} \cdot s^{\prime}=$ $\left\langle s s^{\prime}\right\rangle^{m-2} \boldsymbol{x}$, так что $s^{+}-$последняя буква в словах $\left\langle s s^{\prime}\right\rangle^{m-1}$ и $\left\langle s^{\prime} s\right\rangle^{m}$. Значит, $s^{+}=s$, если $m$ четно, и $s^{+}=s^{\prime}$, если $m$ нечетно.

Коммутационные соотношения гарантируют, что мы можем передвигать буквы в подслове $\left\langle s^{\prime} s\right\rangle^{m}$ одну за другой с левой стороны от $x$ направо, получая соотношения $t=\left\langle s^{\prime} s\right\rangle^{m} x=\left\langle s^{\prime} s\right\rangle^{m-1} x s^{\prime}=\left\langle s^{\prime} s\right\rangle^{m-2} x s s^{\prime}$ и т. д.

Рассмотрим подслучай, когда $m$ четно, т. е. $m=2 n$. Передвинем половину слова $\left\langle s^{\prime} s\right\rangle^{m}$ и получим $t=\left\langle s^{\prime} s\right\rangle^{n} * x$. В частности, $x$ является квазиотражением, $x \in \mathcal{T}(\mathcal{S})$. Кроме того, $t^{\prime}=s^{\prime} t s^{\prime}=\left\langle s s^{\prime}\right\rangle^{n-1} * x$. Сравнивая длину и ширину $t^{\prime}$ и $x$, получаем соотношение $\ell\left(t^{\prime}\right)=\ell(x)+2 \ell\left(\left\langle s s^{\prime}\right\rangle^{n-1}\right)$. Посколькy $\operatorname{wd}\left(t^{\prime}\right)<k$, можно применить предположение индукции и получить, что $\operatorname{wd}(x)=\operatorname{wd}\left(t^{\prime}\right)-(n-1)=k-n$. В частности, элемент $x$ может быть задан выражением вида $x=\boldsymbol{w} s_{0} \boldsymbol{w}^{-1}$, где $s_{0} \in \mathcal{S}$, а $\boldsymbol{w}$ - приведенное слово длины $\ell(\boldsymbol{w})=k-n$. Теперь, используя идемпотентность $x=x^{-1}$, мы можем переписать соотношение коммутативности $s^{\prime} x=x s$ в виде $x s x s^{\prime}=1$. Следовательно, мы можем применить сильное свойство замены к выражению $x \cdot\left(s \boldsymbol{w} s_{0} \boldsymbol{w}^{-1} s^{\prime}\right)$. Это означает, что $x$ сокращается с некоторой буквой в слове $s \boldsymbol{w} s_{0} \boldsymbol{w}^{-1} s^{\prime}$ и значение полученного слова в группе $\mathrm{W}(\mathcal{S})$ равно 1. Сокращающаяся буква не может быть первой буквой $s$, поскольку тогда $x=s$ и $s^{\prime}=x s x=s$, что противоречит предположению $s \neq s^{\prime}$. Эта буква не может быть $s_{0}$ в середине, поскольку тогда мы получили бы, что $s s^{\prime}=1$, откуда снова имели бы $s=s^{\prime}$. Итак, сокращающаяся буква лежит либо в слове $\boldsymbol{w}$, либо в слове $\boldsymbol{w}^{-1}$. Рассмотрим первую возможность. Тогда $\boldsymbol{w}$ имеет вид $\boldsymbol{w}=\boldsymbol{w}^{\prime} s_{1} \boldsymbol{w}^{\prime \prime}$, где $s_{1}$ - это сокращающаяся буква, а $\boldsymbol{w}^{\prime}, \boldsymbol{w}^{\prime \prime}$ - подслова в $\boldsymbol{w}$. Сокращение $x$ и $s_{1}$ эквивалентно соотношению $x=\boldsymbol{w}^{\prime} * s_{1}$. Отсюда следует, что квазиотражение $x$ имеет ширину $\operatorname{wd}(x) \leqslant \ell\left(\boldsymbol{w}^{\prime}\right)<\ell(\boldsymbol{w})$, что противоречит $\ell(\boldsymbol{w})=\operatorname{wd}(x)$. Итак, сокращающаяся буква должна лежать в слове $\boldsymbol{w}^{-1}$. Как и выше, это означает, что $\boldsymbol{w}^{-1}=\boldsymbol{w}^{\prime} s_{1} \boldsymbol{w}^{\prime \prime}$, где $s_{1}-$ сокращающаяся буква и $\boldsymbol{w}^{\prime}, \boldsymbol{w}^{\prime \prime}-$ подслова в $\boldsymbol{w}^{-1}$. 
Заметим теперь, что $x s x s^{\prime}=1=s x s^{\prime} x$. Поэтому сокращение означает выполнение соотношения $x=\left(\boldsymbol{w}^{\prime \prime}\right)^{-1} s_{1} \boldsymbol{w}^{\prime \prime}=\left(\left(\boldsymbol{w}^{\prime \prime}\right)^{-1}\right) * s_{1}$. Итак, в этом случае мы также приходим к противоречию. Значит, $m$ не может быть четным в условиях рассматриваемого случая.

Если $m$ нечетно, т.е. $m=2 n+1$, то применимо аналогичное рассуждение. А именно, начнем с выражения $t=\left\langle s^{\prime} s\right\rangle^{m} x=\left\langle s^{\prime} s\right\rangle^{2 n+1} x$ и передвинем $n$ букв направо от $x$. Это дает выражение $t=\left\langle s^{\prime} s\right\rangle^{n} *\left(s^{\#} x\right)$, где $s^{\#}$ есть $s$ или $s^{\prime}$, в зависимости от четности $n$, откуда

$$
t^{\prime}=\left\langle s s^{\prime}\right\rangle^{n-1} *\left(s^{\#} x\right), \quad \ell\left(t^{\prime}\right)=\ell\left(s^{\#} x\right)+2 \ell\left(\left\langle s s^{\prime}\right\rangle^{n-1}\right) .
$$

Как и выше, мы заключаем, что $s^{\#} x$ является квазиотражением ширины $\operatorname{wd}\left(s^{\#} x\right)=k-n$. Следовательно, $s^{\#} x$ имеет вид $s^{\#} t^{\#} s^{\#}$ для некоторого другого квазиотражения $t^{\#}$ ширины $\operatorname{wd}\left(t^{\#}\right)=k-n-1$. В частности, $\ell\left(s^{\#} t^{\#} s^{\#}\right)=\ell\left(t^{\#}\right)+2$. Напомним теперь, что при нечетном $m=2 n+1$ элемент $x$ коммутирует с $s$ и с $s^{\prime}$, и поскольку $s^{\#}-$ одна из этих образующих, мы получаем $s^{\#} t^{\#} s^{\#}=t^{\#}$. Последнее равенство противоречит соотношению $\ell\left(s^{\#} t^{\#} s^{\#}\right)=\ell\left(t^{\#}\right)+2$. Тем самым, $m$ не может быть нечетным в условиях рассматриваемого случая. Итак, соотношение $\ell(x s)=\ell\left(x s^{\prime}\right)=\ell(x)+1$ вообще не реализуется.

Случай $(\mathrm{c}): \ell\left(x s^{\prime}\right)=\ell(x)-1$ и $\ell(x s)=\ell(x)+1$. Тогда $x=y s^{\prime}$ для некоторого элемента $y \in \mathrm{W}(\mathcal{S})$ длины $\ell(y)=\ell(x)-1$. Поскольку $\ell(t s)=\ell\left((s t)^{-1}\right)=$ $\ell(s t)=\ell(t)-1$, мы можем применить сильное свойство замены к произведению $t \cdot s=\left\langle s^{\prime} s\right\rangle^{m} \boldsymbol{x} \cdot s$. Последняя буква $s$ не может сокращаться с буквой из слова $\boldsymbol{x}$ или с внутренней буквой из подслова $\left\langle s^{\prime} s\right\rangle^{m}$. Поэтому единственные возможности - это сокращения первой и последней букв в $\left\langle s^{\prime} s\right\rangle^{m}$. Далее, после сокращения мы получаем либо $\left\langle s^{\prime} s\right\rangle^{m-1} \boldsymbol{x}$, либо $\left\langle s s^{\prime}\right\rangle^{m-1} \boldsymbol{x}$ в качестве приведенного слова, представляющего произведение $t \cdot s$, значит, $\left\langle s^{\prime} s\right\rangle^{m-1} \boldsymbol{x} s$ или $\left\langle s s^{\prime}\right\rangle^{m-1} \boldsymbol{x} s$ в качестве приведенного слова, представляющего $t$. В любом случае выполнено коммутационное соотношение $x s=s^{*} x$, в котором $s^{*}-$ последняя буква либо в слове $\left\langle s^{\prime} s\right\rangle^{m}$, либо в слове $\left\langle s s^{\prime}\right\rangle^{m}$.

Рассмотрим сначала подслучай, в котором первая буква в подсловах $\left\langle s s^{\prime}\right\rangle^{m-1}$, $\left\langle s s^{\prime}\right\rangle^{m}$ есть $s$, так что $t=\left\langle s s^{\prime}\right\rangle^{m-1} x s$. Подставляя $x=y s^{\prime}$, мы получаем выражения $t=\left\langle s s^{\prime}\right\rangle^{m} y s^{\prime}=\left\langle s s^{\prime}\right\rangle^{m-1} s^{*} y s^{\prime}=\left\langle s s^{\prime}\right\rangle^{m-1} y s^{\prime} s$ и, значит, $s^{*} y s^{\prime}=y s^{\prime} s$. По лемме 2.4 имеем $y s^{\prime} s=z\left\langle s s^{\prime}\right\rangle^{m}=z\left\langle s^{\prime} s\right\rangle^{m}$ для какого-то $z \in \mathrm{W}(\mathcal{S})$ длины $\ell(z)=\ell(y)-(m-2)$. Из двух возможных слов $\left\langle s^{\prime} s\right\rangle^{m},\left\langle s s^{\prime}\right\rangle^{m}$, дающих один и тот же элемент, выберем заканчивающееся на $s$; оно может быть записано в виде $\left(\left\langle s s^{\prime}\right\rangle^{m}\right)^{-1}$. Тогда $x s=y s^{\prime} s=z\left(\left\langle s s^{\prime}\right\rangle^{m}\right)^{-1}=z\left(\left\langle s^{\prime} s\right\rangle^{m-1}\right)^{-1} s$. Коммутационное соотношение $x s=s^{*} x$ можно переписать в форме $s^{*} x=z\left\langle s s^{\prime}\right\rangle^{m}=$ $s^{*} z\left(\left\langle s^{\prime} s\right\rangle^{m-1}\right)^{-1}$. Окончательно получаем

$$
\begin{aligned}
t & =\left\langle s s^{\prime}\right\rangle^{m-1} s^{*} y s^{\prime}=\left\langle s s^{\prime}\right\rangle^{m-1} s^{*} z\left(\left\langle s^{\prime} s\right\rangle^{m-1}\right)^{-1}=\left\langle s s^{\prime}\right\rangle^{m} z\left(\left\langle s^{\prime} s\right\rangle^{m-1}\right)^{-1} \\
& =\left\langle s^{\prime} s\right\rangle^{m} z\left(\left\langle s^{\prime} s\right\rangle^{m-1}\right)^{-1}=\left\langle s^{\prime} s\right\rangle^{m-1}\left(s^{+} z\right)\left(\left\langle s^{\prime} s\right\rangle^{m-1}\right)^{-1} \\
& =\left\langle s^{\prime} s\right\rangle^{m-1} *\left(s^{+} z\right),
\end{aligned}
$$

где $s^{+}$- это элемент из пары $\left(s, s^{\prime}\right)$, отличный от $s^{*}$. Подставляя тождество $t^{\prime}=s^{\prime} t s^{\prime}$ в соотношение (2.2), мы получаем выражение $t^{\prime}=\left\langle s s^{\prime}\right\rangle^{m-2} *\left(s^{+} z\right)$, 
согласованное с длиной, т. е. $\ell\left(t^{\prime}\right)=\ell\left(s^{+} z\right)+2(m-2)$. Теперь, как в случаях (а) и (b), мы заключаем, что элемент $s^{+} z=: t^{+}$является квазиотражением ширины $\operatorname{wd}\left(t^{+}\right)=\operatorname{wd}\left(t^{\prime}\right)-(m-2)=\operatorname{wd}(t)-(m-1)$ и длины $\ell\left(t^{+}\right)=\ell(t)-2(m-1)$. Кроме того, сравнивая длины, мы получаем $\ell\left(t^{+}\right)=\ell(z)+1$. По предположению индукции $t^{+}=s^{+} t^{\#} s^{+}$для некоторого квазиотражения $t^{\#}$ ширины $\operatorname{wd}\left(t^{\#}\right)=\operatorname{wd}\left(t^{+}\right)-1$ и длины $\ell\left(t^{\#}\right)=\ell\left(t^{+}\right)-2$. Значит, $t=\left\langle s s^{\prime}\right\rangle^{m} * t^{\#}$ и $\ell(t)=\ell\left(t^{\#}\right)+2 m$. В частности, $x=t^{\#}\left\langle s s^{\prime}\right\rangle^{m}$. Но слово $\left\langle s s^{\prime}\right\rangle^{m} s$ не приведено, и потому $\ell(x s)=\ell\left(t^{\#}\left\langle s s^{\prime}\right\rangle^{m}\right)=\ell\left(t^{\#}\right)+m-1=\ell(x)-1$, что противоречит сделанным предположениям. Итак, подслучай $t=\left\langle s s^{\prime}\right\rangle^{m-1} x s$ невозможен.

Полученное противоречие показывает, что $t=\left\langle s^{\prime} s\right\rangle^{m-1} x s$. Переставляя $s$ и $s^{\prime}$, мы можем использовать рассуждение из предыдущего подслучая вплоть до равенства $t=\left\langle s^{\prime} s\right\rangle^{m-1} s^{*} z\left(\left\langle s^{\prime} s\right\rangle^{m-1}\right)^{-1}$ в формуле (2.2). Перепишем его в виде $t=\left\langle s^{\prime} s\right\rangle^{m-1} *\left(s^{*} z\right)$, пропустим дальнейшие выкладки в формуле (2.2) и продолжим рассуждение. Это дает аналогичное соотношение $t^{\prime}=\left\langle s s^{\prime}\right\rangle^{m-2} *\left(s^{*} z\right)$ для $\ell\left(t^{\prime}\right)=\ell\left(s^{*} z\right)+2(m-2)$ и показывает, что элемент $s^{*} z=: t^{+}$является квазиотражением ширины $\operatorname{wd}\left(t^{+}\right)=\operatorname{wd}\left(t^{\prime}\right)-(m-2)=\operatorname{wd}(t)-(m-1)$ и длины $\ell\left(t^{+}\right)=\ell(t)-2(m-1)$. Поскольку для $t^{+}$выполнено аналогичное равенство $t^{+}=s^{*} z$, где $\ell\left(t^{+}\right)=\ell(z)+1$, квазиотражение $t^{\#}:=s^{*} t^{+} s^{*}$ имеет длину $\ell\left(t^{\#}\right)=\ell\left(t^{+}\right)-2=\ell(t)-2 m$, и мы приходим к тому же противоречию, что и в предыдущем подслучае. Окончательно мы получаем, что весь рассматриваемый случай, в котором $\ell\left(x s^{\prime}\right)=\ell(x)-1$ и $\ell(x s)=\ell(x)+1$, невозможен.

Случай $(\mathrm{d}): \ell\left(x s^{\prime}\right)=\ell(x)+1$ и $\ell(x s)=\ell(x)-1$. Этот случай отличается от случая (с) только тем, что $s$ и $s^{\prime}$ меняются местами, и использованное выше рассуждение применяется с соответствующими минимальными изменениями. На самом деле, этот случай даже легче, так как один из возникающих в случае (с) подслучаев можно исключить более просто. А именно, поскольку $\ell\left(t^{\prime}\right)=\ell\left(s^{\prime} t s^{\prime}\right)=\ell(t)-2$, после сокращений в произведении $\left\langle s^{\prime} s\right\rangle^{m} \boldsymbol{x} \cdot s^{\prime}$ мы можем получить только выражение $\left\langle s^{\prime} s\right\rangle^{m-1} \boldsymbol{x}$.

iii) Утверждение следует непосредственно из доказательства части іi).

iv) Доказательство проводится индукцией по ширине $\mathrm{wd}(t)$ квазиотражений. В случае $\operatorname{wd}(t)=0$ доказательство очевидно, так как $t$ - одна из образующих $s \in \mathcal{S}$. Пусть $t$ - квазиотражение ширины $\operatorname{wd}(t)=: k \geqslant 1$, и предположим, что наше утверждение верно для всех квазиотражений $t^{\prime}$ ширины $\operatorname{wd}\left(t^{\prime}\right)<\operatorname{wd}(t)$. Зафиксируем два приведенных выражения $t=w * s_{0}=w^{\prime} * s_{0}^{\prime}$ при $s_{0}, s_{0}^{\prime} \in \mathcal{S}$ и $w, w^{\prime} \in \mathrm{W}(\mathcal{S})$ длины $\ell(w)=\ell\left(w^{\prime}\right)=k$. Найдем образующие $s, s^{\prime} \in \mathcal{S}$, для которых $\ell(s w)=\ell\left(s^{\prime} w^{\prime}\right)=\ell(w)-1=\ell\left(w^{\prime}\right)-1$, и положим $w_{1}:=s w$ и $w_{1}^{\prime}:=s^{\prime} w^{\prime}$.

Случай $s=s^{\prime}$. В этом случае выражения $w_{1} * s_{0}$ и $w_{1}^{\prime} * s_{0}^{\prime}$ представляют один и тот же элемент в группе $\mathrm{W}(\mathcal{S})$, скажем $t_{1}$, который имеет ширину $\operatorname{wd}\left(t_{1}\right)=k-1$, и, значит, $\widehat{w}_{1} * s_{0}$ и $\widehat{w}_{1}^{\prime} * s_{0}^{\prime}$ сопряжены в $\operatorname{Br}(\mathcal{S})$ элементами $\widehat{w}_{j} * s_{j}^{2}$ при $\ell\left(w_{j}\right)<k-1$, $\widehat{w}_{1} * s_{0}=\left(\prod_{j} \widehat{w}_{j} * s_{j}^{ \pm 2}\right) *\left(\widehat{w}_{1}^{\prime} * s_{0}^{\prime}\right)$. Сопрягая элементом $s=s^{\prime}$, мы получаем соотношение

$$
\begin{aligned}
\widehat{w} * s_{0} & =s *\left(w_{1} * s_{0}\right)=\left(\prod_{j}\left(s \cdot \widehat{w}_{j}\right) * s_{j}^{ \pm 2}\right) *\left(\left(s \cdot \widehat{w}_{1}^{\prime}\right) * s_{0}^{\prime}\right) \\
& =\left(\prod_{j}\left(s \cdot \widehat{w}_{j}\right) * s_{j}^{ \pm 2}\right) *\left(\widehat{w}^{\prime} * s_{0}^{\prime}\right) .
\end{aligned}
$$


Сопрягающие элементы $\left(s \cdot \widehat{w}_{j}\right) * s_{j}^{ \pm 2}$ в этой формуле обладают следующими свойствами. В случае $\ell\left(s \cdot w_{j}\right)=\ell\left(w_{j}\right)+1$ выполнено $\ell\left(s \cdot w_{j}\right)<k$ и $\left(s \cdot w_{j}\right)^{\wedge}=s \cdot \widehat{w}_{j}$, так что выражение $\left(s \cdot \widehat{w}_{j}\right) * s_{j}^{ \pm 2}$ имеет требуемый вид. В случае $\ell\left(s \cdot w_{j}\right)=$ $\ell\left(w_{j}\right)-1$ определим $w_{j}^{\prime}:=s \cdot w_{j}$ как элемент в группе $\mathrm{W}(\mathcal{S})$ и воспользуемся соотношениями $w_{j}:=s \cdot w_{j}^{\prime}$ и $\ell\left(w_{j}\right)=\ell\left(w_{j}^{\prime}\right)+1$. Тогда $\widehat{w}_{j}=s \cdot \widehat{w}_{j}^{\prime}$, поэтому $s \cdot \widehat{w}_{j}=s^{2} \cdot \widehat{w}_{j}^{\prime}$ и, наконец, $\left(s \cdot \widehat{w}_{j}\right) * s_{j}^{ \pm 2}=s^{2} \cdot\left(\widehat{w}_{j}^{\prime} * s_{j}^{ \pm 2}\right) \cdot s^{-2}$. Отсюда следует утверждение iv) в случае $s=s^{\prime}$.

Случай $s \neq s^{\prime}$. Положим $m:=m_{s, s^{\prime}}$. Тогда в силу утверждения iii) имеются две возможности: либо $m$ нечетно, $m=2 n+1$, и $t \equiv\left\langle s s^{\prime}\right\rangle^{m}$, либо квазиотражение $t$ может быть представлено в виде $t \equiv\left\langle s s^{\prime}\right\rangle^{m} * t^{+}$для другого квазиотражения $t^{+}$ширины $\operatorname{wd}\left(t^{+}\right)=\operatorname{wd}(t)-m$. В первом случае существуют только два приведенных слова вида $\boldsymbol{w} * s$, представляющие $t$, а именно $\left\langle s s^{\prime}\right\rangle^{2 n+1} \equiv\left\langle s s^{\prime}\right\rangle^{n} * s^{\#}$ и $\left\langle s^{\prime} s\right\rangle^{2 n+1} \equiv\left\langle s^{\prime} s\right\rangle^{n} * s^{\dagger}$, где $s^{\#}=s$ и $s^{\dagger}=s^{\prime}$, если $n$ четно, и $s^{\#}=s^{\prime}$ и $s^{\dagger}=s$, если $n$ нечетно. Сравнивая подъемы в $\operatorname{Br}(\mathcal{S})$, мы видим, что соотношение в группе кос $\left\langle s s^{\prime}\right\rangle^{2 n+1}=\left\langle s^{\prime} s\right\rangle^{2 n+1}$ эквивалентно в $\operatorname{Br}(\mathcal{S})$ соотношениям $\left\langle s s^{\prime}\right\rangle^{n} * s^{\#}=\left\langle\left(s^{\prime}\right)^{-1} s^{-1}\right\rangle^{n} s^{\dagger}$ и $\left\langle s^{\prime} s\right\rangle^{n} * s^{\dagger}=\left\langle s^{-1}\left(s^{\prime}\right)^{-1}\right\rangle^{n} * s^{\#}$. Остается заметить, что обе пары элементов $\left\langle s s^{\prime}\right\rangle^{n} * s^{\#},\left\langle s^{-1}\left(s^{\prime}\right)^{-1}\right\rangle^{n} * s^{\#}$ и $\left\langle s^{\prime} s\right\rangle^{n} * s^{\dagger}$, $\left\langle\left(s^{\prime}\right)^{-1} s^{-1}\right\rangle^{n} s^{\dagger}$ удовлетворяют iv).

В оставшемся случае $t \equiv\left\langle s s^{\prime}\right\rangle^{m} * t^{+}$выберем представление квазиотражения $t^{+}$в виде $t^{+} \equiv \boldsymbol{w}^{+} * s^{+}$, где $\boldsymbol{w}^{+}-$приведенное слово длины $\ell\left(\boldsymbol{w}^{+}\right)=$ $w \mathrm{~d}(t)-m$. Это дает нам два дополнительных представления квазиотражения $t$ в группе $\mathrm{W}(\mathcal{S})$, а именно $t \equiv\left(\left\langle s s^{\prime}\right\rangle^{m} \boldsymbol{w}^{+}\right) * s^{+} \equiv\left(\left\langle s^{\prime} s\right\rangle^{m} \boldsymbol{w}^{+}\right) * s^{+}$. По лемме 2.5 соответствующие элементы $\left(\left\langle s s^{\prime}\right\rangle^{m} \widehat{\boldsymbol{w}}^{+}\right) * s^{+}$и $\left(\left\langle s^{\prime} s\right\rangle^{m} \widehat{\boldsymbol{w}}^{+}\right) * s^{+}$равны в группе кос $\operatorname{Br}(\mathcal{S})$. Теперь мы можем воспользоваться уже рассмотренным случаем $s=s^{\prime}$ и завершить доказательство.

v) Это утверждение следует из утверждения iv).

Предъявим теперь процедуру, позволяющую описать возможные разложения данного элемента $a \in \mathrm{P}(\mathcal{S})$ в виде произведения RS-образующих $\tilde{t}_{i}^{2}=$ $\widehat{w}_{i} * s_{i}^{2} \in \widetilde{\mathcal{T}}^{2}(\mathcal{S})$.

ОПРеДЕЛЕНИЕ 2.12. Разложением длины $l$ элемента $x$ группы $G$ называется выражение $\boldsymbol{f}=f_{1} \cdot f_{2} \cdots f_{l}$, значение которого в группе $G$ равно $x$. Движением Гурвица называется преобразование такого выражения $\boldsymbol{f}=f_{1} \cdot f_{2} \cdots f_{l}$, при котором пара сомножителей $f_{i} \cdot f_{i+1}$ заменяется на $\left(f_{i} f_{i+1} f_{i}^{-1}\right) \cdot f_{i}$ или на $f_{i+1} \cdot\left(f_{i+1}^{-1} f_{i} f_{i+1}\right)$, а остальные сомножители остаются неизменными. Мы будем говорить, что сомножитель $f_{i}$ сдвигается (без сопряжения) вправо, а сомножитель $f_{i+1}$ сдвигается с сопряжением влево, или, соответственно, что $f_{i}$ сдвигается с сопряжением вправо, а $f_{i+1}$ сдвигается без сопряжения влево.

Преобразованием Гурвица называется последовательность движений Гурвица. Два разложения $\boldsymbol{f}$ и $\boldsymbol{f}^{\prime}$, получающиеся друг из друга преобразованием Гурвица, называются гурвиц-эквивалентными.

Например, применяя подходящую последовательность движений Гурвица, мы можем сдвинуть заданный сомножитель $f_{k}$ на правый конец разложения. Полученное разложение имеет вид $f_{k} \cdot f_{1}^{\prime} \cdots f_{k-1}^{\prime} \cdot f_{k+1} \cdots f_{l}$ с сопряженными сомножителями $f_{i}^{\prime}=f_{k}^{-1} f_{i} f_{k}, i=1, \ldots, k-1$. (Подробнее об этих понятиях см., например, работу [32].) 
ТЕОРема 2.13 (проблема Гурвица в группах Кокстера-Вейля). Пусть $\boldsymbol{t}=$ $t_{1} \cdot t_{2} \cdots t_{l}-$ разложение единицы $1 \in \mathrm{W}(\mathcal{S})$ в произведение квазиотражений $t_{i} \in \mathcal{T}(\mathcal{S})$. Тогда длина $l$ этого разложения четна, $l=2 l^{\prime}$, и разложение $\boldsymbol{t}$ гурвиц-эквивалентно разложению $\boldsymbol{t}^{\prime}$ в произведение квадратов квазиотражений, т.е. разложению вида $\boldsymbol{t}^{\prime}=t_{1}^{\prime} \cdot t_{2}^{\prime} \cdots t_{l}^{\prime}$, где $t_{2 i-1}^{\prime} \equiv t_{2 i}^{\prime} \in \mathcal{T}(\mathcal{S})$.

В специальном случае конечных групп Вейля, соответствующих простым комплексным алгебрам Ли, этот результат был получен Каневым [31, предложение 2.3]. Доказательство Канева использует другие методы, а именно геометрию и комбинаторику соответствующей системы корней.

ДоКАЗАТЕЛЬСТВО ТЕОРЕМЫ 2.13. Поскольку множество квазиотражений $\mathcal{T}(\mathcal{S})$ инвариантно относительно сопряжений, всякое преобразование Гурвица переводит $\boldsymbol{t}$ в разложение в произведение квазиотражений.

Сдвигая с сопряжением первый сомножитель $t_{1}$ на правый конец разложения, мы получаем разложение вида $t_{2} \cdot t_{3} \cdots t_{l} \cdot \tilde{t}_{1}$. Сравнивая его с исходным разложением $\boldsymbol{t}=t_{1} \cdot t_{2} \cdots t_{l} \equiv 1$, мы получаем равенство $\tilde{t}_{1} \equiv t_{1}$. Следовательно, циклическая перестановка сомножителей в разложении $\boldsymbol{t}$ приводит к гурвиц-эквивалентным разложениям. Поэтому мы можем считать, что последний сомножитель $t_{l}$ имеет наибольшую ширину среди всех $t_{i}$.

Представим теперь каждый элемент $t_{i}, i=1, \ldots, l-1$, в виде $t_{i} \equiv w_{i} * s_{i}$ с наименьшей возможной длиной $\ell\left(w_{i}\right)$. Зафиксируем приведенное выражение $\boldsymbol{w}_{i}$ для каждого $w_{i}$. Заметим, что обращение выражения ${ }^{1} \boldsymbol{w}$ любого элемента $w \in \mathrm{W}(\mathcal{S})$ дает выражение для обратного элемента $w^{-1}$. С учетом этого обозначим через $\boldsymbol{w}^{-1}$ обращение выражения $\boldsymbol{w}$.

Рассмотрим выражение $\boldsymbol{w}:=\prod_{i=1}^{l-1} \boldsymbol{w}_{i} \cdot s_{i} \cdot \boldsymbol{w}_{i}^{-1}$. Его значение в группе $\mathrm{W}(\mathcal{S})$ есть $t_{l}^{-1} \equiv t_{l}$, а его произведение с $t_{l}$ равно 1 . По сильному свойству замены найдется буква $s$ в $\boldsymbol{w}$, исключение которой из $\boldsymbol{w}$ дает выражение для 1.

Рассмотрим сначала случай, когда $s-$ средняя буква $s_{j}$ в выражении $\boldsymbol{w}_{j} \cdot s_{j} \cdot \boldsymbol{w}_{j}^{-1}$ для некоторого $t_{j}$. Тогда оставшиеся части $\boldsymbol{w}_{j} \cdot \boldsymbol{w}_{j}^{-1}$ сокращаются и, следовательно,

$$
t_{1} \cdots t_{j-1} \cdot t_{j} \cdot t_{j+1} \cdots t_{l-1} \equiv t_{1} \cdots t_{j-1} \cdot t_{j+1} \cdots t_{l-1} \cdot t_{l}
$$

С другой стороны, сдвигая с сопряжением сомножитель $t_{j}$ в выражении $t_{1} \cdots t_{j-1} \cdot t_{j} \cdot t_{j+1} \cdots t_{l-1}$ на правый конец, мы получаем разложение $t_{1} \cdots t_{j-1} \times$ $t_{j+1} \cdots t_{l-1} \cdot \tilde{t}_{j}$. Следовательно, $\tilde{t}_{j} \equiv t_{l}$, и разложение $t_{1} \cdots t_{j-1} \cdot t_{j} \cdot t_{j+1} \cdots t_{l-1} \cdot t_{l}$ гурвиц-эквивалентно разложению $t_{1} \cdots t_{j-1} \cdot t_{j+1} \cdots t_{l-1} \cdot t_{l} \cdot t_{l}$. Поскольку в этом случае $t_{1} \cdots t_{j-1} \cdot t_{j+1} \cdots t_{l-1} \equiv 1$, мы получаем утверждение теоремы индукцией по $l$.

Остается рассмотреть случай, когда буква $s$ появляется, скажем, в начальном подслове $\boldsymbol{w}_{j}$ в выражении $\boldsymbol{w}_{j} \cdot s_{j} \cdot \boldsymbol{w}_{j}^{-1}$ для какого-то квазиотражения $t_{j}$. (Случай, когда буква $s$ встречается в конечном подслове $\boldsymbol{w}_{j}^{-1}$ рассматривается аналогично.) Запишем слово $\boldsymbol{w}_{j}$ в виде $\boldsymbol{w}^{\prime} s \boldsymbol{w}^{\prime \prime}$. Тогда $\boldsymbol{w}^{\prime} \boldsymbol{w}^{\prime \prime} \equiv$ $\left(\boldsymbol{w}^{\prime} s\left(\boldsymbol{w}^{\prime}\right)^{-1}\right) \cdot \boldsymbol{w}^{\prime} s \boldsymbol{w}^{\prime \prime}$ и, значит,

$$
\boldsymbol{w}^{\prime} \boldsymbol{w}^{\prime \prime} \cdot s_{j} \cdot \boldsymbol{w}_{j}^{-1} \equiv\left(\boldsymbol{w}^{\prime} s\left(\boldsymbol{w}^{\prime}\right)^{-1}\right) \cdot\left(\boldsymbol{w}_{j} s_{j} \boldsymbol{w}_{j}^{-1}\right) .
$$

\footnotetext{
${ }^{1}$ Обращение порядка букв в слове $\boldsymbol{w}$.
} 
Сдвинем теперь $t_{l}$ с сопряжением влево до положения между $t_{j-1}$ и $t_{j}$. Это дает разложение вида $t_{1} \cdots t_{j-1} \cdot \tilde{t}_{l} \cdot t_{j} \cdot t_{j+1} \cdots t_{l-1}$. Сравнивая его с равенством $(2.3)$, мы получаем равенство $\tilde{t}_{l} \equiv \boldsymbol{w}^{\prime} s\left(\boldsymbol{w}^{\prime}\right)^{-1}$. Заметим, что ширина квазиотражения $\tilde{t}_{l} \equiv \boldsymbol{w}^{\prime} s\left(\boldsymbol{w}^{\prime}\right)^{-1}$ не превосходит $\ell\left(\boldsymbol{w}^{\prime}\right)<\ell\left(\boldsymbol{w}^{\prime} s \boldsymbol{w}^{\prime \prime}\right)$ и, следовательно, меньше ширины квазиотражения $t_{l}$. Теперь можно провести индукцию по сумме ширин квазиотражений $t_{i}$. Теорема доказана.

Рассмотрим теперь следующую ситуацию. Пусть $G$ - подгруппа в $\mathrm{W}(\mathcal{S})$. Тогда группа $G$ действует на $\mathrm{P}_{\mathrm{ab}}(\mathcal{S})$ сопряжением, и мы обозначим через $\mathrm{P}_{\text {ab }}(\mathcal{S})_{G}$ группу коинвариантов (см., например, [14]). Напомним, что $\mathrm{P}_{\mathrm{ab}}(\mathcal{S})_{G}$ является фактором группы $\mathrm{P}_{\mathrm{ab}}(\mathcal{S})$ по подгруппе, порожденной элементами вида $w * A-A$, где $w \in G$ и $A \in \mathrm{P}_{\mathrm{ab}}(\mathcal{S})$. Поскольку $\mathrm{W}(\mathcal{S})$ переставляет элементы базиса в $\mathrm{P}_{\mathrm{ab}}(\mathcal{S})$, группа $\mathrm{P}_{\mathrm{ab}}(\mathcal{S})_{G}$ является свободной абелевой группой с базисом, задаваемым фактормножеством $\mathcal{T}(\mathcal{S}) / G$. По-другому группу $\mathrm{P}_{\text {ab }}(\mathcal{S})_{G}$ можно описать как фактор группы $\mathrm{P}(\mathcal{S})$ по подгруппе $[\widetilde{G}, \mathrm{P}(\mathcal{S})]$, где $\widetilde{G}$ - прообраз группы $G$ в группе $\operatorname{Br}(\mathcal{S})$. Мы будем использовать обозначение $G \cdot t$ для элементов множества $\mathcal{T}(\mathcal{S}) / G$ и обозначение $A_{G \cdot \theta}$ или $A_{G \cdot t}$ для элементов индуцированного базиса в группе $\mathrm{P}_{\mathrm{ab}}(\mathcal{S})_{G}$.

Теорема 2.14. Пусть $G$ - подгруппа в $\mathrm{W}(\mathcal{S})$ и $x$ - элемент группъ $\mathrm{P}(\mathcal{S})$, представимый в виде произведения: $x=\prod_{i} \hat{t}_{i}^{\epsilon_{i}} \cdot \prod_{j}\left[x_{2 j-1}, x_{2 j}\right]$, в котором $\hat{t}_{i}-$ квазиобразующие, $\epsilon_{i}= \pm 1, a\left[x_{2 j-1}, x_{2 j}\right]$ - коммутаторы элементов $x_{j} \in \operatorname{Br}(\mathcal{S})$. Предположим, что образы элементов $x_{i}$ и $\hat{t}_{j}$ в группе $\mathrm{W}(\mathcal{S})$ лежат в $G$. Тогда образ $[x]_{G}$ элемента $x$ в группе $\mathrm{P}_{\mathrm{ab}}(\mathcal{S})_{G}$ лежит в свободной абелевой группе, порожденной базисными элементами $A_{G \cdot t} \partial л я t \in G \cap \mathcal{T}(\mathcal{S})$.

ДокАЗАтЕльство. Сначала сведем общий случай к специальному случаю отсутствия коммутаторов. Для этого представим каждый элемент $x_{j}$ в виде $x_{j}=x_{j}^{\prime} \cdot x_{j}^{\prime \prime}$, где $x_{j}^{\prime}$ - произведение квазиобразующих, проекции которых лежат в $G$, а $x_{j}^{\prime \prime}$ - произведение квадратов квазиобразующих. Используя тождества $[x, y \cdot z]=[x, y] \cdot(y *[x, z])$ и $[x \cdot y, z]=(x *[y, z]) \cdot[x, z]$, разложим каждый коммутатор $\left[x_{2 i-1}, x_{2 i}\right]$ в произведение квазиобразующих, проектирующихся в $G$, и коммутаторов вида $\left[y, \hat{t}^{2}\right]$, где элемент $y$ проектируется в $G$, а $\hat{t}^{2}-$ квадрат квазиобразующей. Поскольку такой коммутатор $\left[y, \hat{t}^{2}\right]$ лежит в $\mathrm{P}(\mathcal{S})$ и отображается в нуль в группе $\mathrm{P}_{\mathrm{ab}}(\mathcal{S})_{G}$, мы получаем нужное утверждение.

Заметим, что движения Гурвица не нарушают свойства сомножителей $\hat{t}_{i}^{\epsilon_{i}}$, перечисленные в условии теоремы. Поэтому применяя теорему 2.13, мы можем преобразовать исходное разложение так, чтобы выполнялось равенство $t_{2 i-1} \equiv$ $t_{2 i} \in \mathrm{W}(\mathcal{S})$. Это означает, что достаточно рассмотреть случай, когда $x$ является произведением двуx сомножителей, $x=\hat{t}_{1}^{\epsilon_{1}} \cdot \hat{t}_{2}^{\epsilon_{2}}$, в котором $t_{1} \equiv t_{2}$. В случае, когда знаки $\epsilon_{i}$ совпадают, например $\epsilon_{1}=\epsilon_{2}=+1$, мы можем переписать это произведение в виде $\hat{t}_{1} \hat{t}_{2}^{-1} \hat{t}_{2}^{2}$. Поскольку $t_{2} \in G \cap \mathcal{T}(\mathcal{S})$ по условию, квадрат $\hat{t}_{2}^{2}$ имеет нужный вид. Итак, мы можем дополнительно предполагать, что $x=$ $\hat{t}_{1} \cdot \hat{t}_{2}^{-1}$.

Мы утверждаем, что из условия $t_{1} \equiv t_{2} \in \mathrm{W}(\mathcal{S})$ следует, что элемент $\hat{t}_{2}$ может быть получен из элемента $\hat{t}_{1}$ сопряжением некоторым элементом $z \in \mathrm{P}(\mathcal{S})$. Чтобы это доказать, запишем $\hat{t}_{i}=y_{i} * s_{i}$, где $s_{i} \in \mathcal{S}$ и $y_{i} \in \operatorname{Br}(\mathcal{S})$, и сопряжем оба элемента $\hat{t}_{i}$ элементом $y_{1}^{-1}$. Это сводит ситуацию к частному случаю, 
в котором $\hat{t}_{1}=s_{1}$ - обычная образующая группы $\operatorname{Br}(\mathcal{S})$. Изменяя соответствующим образом обозначения, имеем $\hat{t}_{2}=y_{2} * s_{2}$. Пусть $w$ - образ элемента $y_{2}$ в группе $\mathrm{W}(\mathcal{S})$. Наше утверждение будет следовать из того, что в группе кос имеет место равенство $\widehat{w} * s_{2}=s_{1}$. Докажем этот факт. Предположим сначала, что $\ell\left(w s_{2}\right)<\ell(w)$. Тогда по сильному свойству замены $w \equiv w^{\prime} s_{2}$, где $\ell\left(w^{\prime}\right)=\ell(w)-1$. В этом случае $\ell\left(w^{\prime} s_{2}\right)>\ell\left(w^{\prime}\right), \widehat{w} * s_{2}=\left(\widehat{w}^{\prime} s_{2}\right) * s_{2}=\widehat{w}^{\prime} * s_{2}$ и по-прежнему $\widehat{w}^{\prime} * s_{2} \equiv s_{1}$ в группе $\mathrm{W}(\mathcal{S})$. Поэтому мы можем считать, что $\ell\left(w s_{2}\right)>\ell(w)$. Заметим, что равенство $w * s_{2} \equiv s_{1}$ эквивалентно равенству $w s_{2} \equiv s_{1} w$. Поскольку $\ell\left(w s_{2}\right)>\ell(w)$, для любого приведенного выражения $\boldsymbol{w}$ элемента $w$ выражения $\boldsymbol{w} s_{2}$ и $s_{1} \boldsymbol{w}$ приведенные. Следовательно, $\widehat{w} \cdot s_{2}=s_{1} \cdot \widehat{w}$ в группе кос. Этим доказаны равенство $\widehat{w} * s_{2}=s_{1}$ и, следовательно, сделанное в начале этого абзаца утверждение.

Подводя итог, нам остается рассмотреть коммутатор $\hat{t} z \hat{t}^{-1} z^{-1}$, где $z \in \mathrm{P}(\mathcal{S})$ и $\hat{t} \in G$. По определению такой элемент проектируется в нуль в группе $\mathrm{P}_{\mathrm{ab}}(\mathcal{S})_{G}$. Теорема доказана.

ОПРЕДЕЛЕНИЕ 2.15. Система Кокстера $(\mathcal{S}, M)$ называется неприводимой, если ее граф Кокстера связен, и имеет конечный mun, если группа $\mathrm{W}(\mathcal{S})$ конечна. Известно, что в любой неприводимой системе Кокстера конечного типа $(\mathcal{S}, M)$ существует единственный элемент максимальной длинь $w_{\circ}=w_{\circ}(\mathcal{S}) \in$ $\mathrm{W}(\mathcal{S})$. Его канонический подъем $\widehat{w}_{\circ} \in \operatorname{Br}(\mathcal{S})$ называется элементом Гарсайда группы $\operatorname{Br}(\mathcal{S})$ и обозначается $\Delta(\mathcal{S})$.

Согласно классификации неприводимых групп Кокстера конечного типа (см. [15] и [27]) всякая такая группа есть либо группа Вейля простой комплексной группы Ли, либо группа диэдра $\mathcal{D}_{2 m}$ с системой Кокстера на множестве $\mathcal{S}=\left\{s_{1}, s_{2}\right\}$, заданной соотношениями $m_{s_{1}, s_{2}}=m, m=5,7,8,9, \ldots$, либо одна из групп $\mathrm{H}_{3}, \mathrm{H}_{4}$. Известно, что элемент максимальной длины $w_{\circ} \in \mathrm{W}(\mathcal{S})$ обладает свойством $\ell\left(w_{\circ}\right)=\ell\left(w_{\circ} w\right)+\ell(w)$ для любого $w \in \mathbf{W}(\mathcal{S})$. Свойства элемента Гарсайда можно найти в [13] и [18].

Лемма 2.16. Kвадрат $\Delta^{2}(\mathcal{S})$ элемента Гарсайда лежит в группе $\mathrm{P}(\mathcal{S})$ и равен сумме $\sum_{t \in \mathcal{T}(\mathcal{S})} A_{t}$ в абелианизации $\mathrm{P}_{\mathrm{ab}}(\mathcal{S})$.

ДокАЗАТЕЛЬСтво. Применяя свойство $\ell\left(w_{\circ} w\right)=\ell\left(w_{\circ}\right)-\ell(w)$ в случае $w=w_{\circ}$, мы получаем, что $w_{\circ}-$ идемпотент. Значит, элемент $\Delta^{2}=\widehat{w}_{\circ}^{2}$ лежит в группе $\mathrm{P}(\mathcal{S})$.

Рассмотрим произвольную образующую $s \in \mathcal{S}$ и любое приведенное выражение $\boldsymbol{w}$ элемента $w_{\circ}$. Тогда $\ell\left(s w_{\circ}\right)=\ell\left(w_{\circ}\right)-\ell(s)<\ell\left(w_{\circ}\right)$. По сильному свойству замены, удалив подходящую букву $s^{\prime}$ из выражения $\boldsymbol{w}$, мы получаем слово $\boldsymbol{w}_{1}$, являющееся выражением элемента $s w_{\circ}$. Сравнивая длины, мы получаем, что $\boldsymbol{w}_{1}$ приведено. Записав $\boldsymbol{w}_{1}$ в виде $s_{2} \cdots s_{l}$, где $l:=\ell\left(w_{\circ}\right)$, мы видим, что $s s_{2} \cdots s_{l}$ является приведенным выражением элемента $w_{\circ}$. Поскольку $w_{\circ}$ идемпотент, то $s_{l} \cdots s_{2} s$ также является приведенным выражением для $w_{\circ}$. Следовательно, $\Delta^{2}=\hat{s} \hat{s}_{2} \cdots \hat{s}_{l} \cdot \hat{s}_{l} \cdots \hat{s}_{2} \hat{s}$. Введем обозначение $s_{1}:=s$ и преобразуем последнее выражение в произведение

$$
\Delta^{2}=\prod_{i=1}^{l}\left(\hat{s}_{1}^{-1} \hat{s}_{2}^{-1} \cdots \hat{s}_{i-1}^{-1}\right) * \hat{s}_{i}^{2} .
$$


Отсюда следует, что в группе $\mathrm{P}_{\mathrm{ab}}(\mathcal{S})$ элемент $\Delta^{2}$ равен сумме $\sum_{t \in \mathcal{T}(\mathcal{S})} n_{t} A_{t}$, где неотрицательные целые числа $n_{t}$ таковы, что $n_{s}>0$ для любого $s \in \mathcal{S}$. Поскольку $\Delta^{2}$ лежит в центре группы $\operatorname{Br}(\mathcal{S})$, сумма $\sum_{t \in \mathcal{T}(\mathcal{S})} n_{t} A_{t}$ инвариантна при действии группы $\mathrm{W}(\mathcal{S})$. Так как это действие переставляет базисные элементы $A_{t}$ группы $\mathrm{P}_{\text {аb }}(\mathcal{S})$, а всякое квазиотражение $t \in \mathcal{T}(\mathcal{S})$ сопряжено образующей $s \in \mathcal{S}$, мы получаем неравенства $n_{t} \geqslant 1$ для всех $t \in \mathcal{T}(\mathcal{S})$.

С другой стороны, в работах [13] и [18] показано, что элемент $\Delta^{2}$ имеет выражение $\Delta^{2}=\widehat{\Pi}^{h}$, где П - это так называемый элемент Kокстера в группе $\mathrm{W}(\mathcal{S})$, а $h$ - число Кокстера системы $\mathcal{S}$ (определения см., например, в [15] или [27]). Формула для числа Кокстера может быть истолкована как равенство длины $l=\ell\left(w_{\circ}\right)$ и количества квазиотражений. Это означает равенство $\sum_{t \in \mathcal{T}(\mathcal{S})} n_{t}=l$. Следовательно, все $n_{t}$ равны 1 , и лемма доказана.

2.2. Комбинаторная структура группы $\mathrm{Map}_{g}$. В этом пункте мы решаем ряд задач о разложении на множители в группе классов отображений. Эти результаты будут играть ключевую роль в доказательстве основной теоремы.

Мы будем использовать найденные Б. Вайнрыбом [42] и М. Мацумото [35] конечные копредставления групп $\mathrm{Map}_{g}$ и $\mathrm{Map}_{g,[1]}$, которые реализуют эти группы как факторы группы кос некоторой системы Кокстера $\mathcal{S}_{g}$, получающиеся добавлением соотношений, задаваемых в терминах элементов Гарсайда подходящих подсистем $\mathcal{S}^{\prime}$ системы $\mathcal{S}_{g}$. Проведем геометрическое описание этих соотношений (подробности можно найти, например, в работах [8] и [22]).

ОПРЕДЕЛЕНИЕ 2.17 (цепное и фонарное соотношения). Рассмотрим поверхность $\mathcal{C}$, являющуюся тором с двумя дырками. Обозначим граничные окружности через $\eta^{\prime}, \eta^{\prime \prime}$. Рассмотрим на этой поверхности кривые $\alpha, \beta$ и $\beta^{\prime}$, показанные на рис. 1. Заметим, что если $\alpha, \beta$ и $\beta^{\prime}$ - вложенные окружности на поверхности $\Sigma$ такие, что $\beta$ и $\beta^{\prime}$ не пересекаются, и каждая из них пересекает $\alpha$ трансверсально ровно в одной точке, то трубчатая окрестность $U$ графа $\alpha \cup \beta \cup \beta^{\prime}$ есть тор с двумя дырками и вся конфигурация диффеоморфна конфигурации, изображенной на рис. 1. Будем называть такую поверхность $\mathcal{C} \subset \Sigma$ и

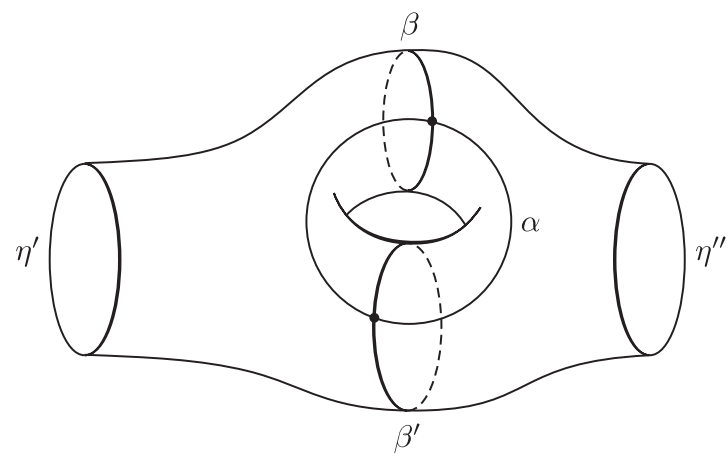

Рис. 1. Окружности в цепной конфигурации

всю конфигурацию $\left(\mathcal{C}, \alpha, \beta, \beta^{\prime}\right)$ цепной конфигурацией на поверхности $\Sigma$, определяемой окружностями $\alpha, \beta, \beta^{\prime}$. Для всякой цепной конфигурации имеется 
цепной элемент группы классов отображений, заданный формулой

$$
C\left(\alpha, \beta, \beta^{\prime}\right):=\left(T_{\beta} T_{\alpha} T_{\beta^{\prime}}\right)^{4}\left(T_{\eta^{\prime}} T_{\eta^{\prime \prime}}\right)^{-1} .
$$

Рассмотрим еще поверхность $\mathcal{L}$, являющуюся сферой с четырьмя дырками. Обозначим ее граничные окружности через $\alpha_{1}, \ldots, \alpha_{4}$. Представим $\mathcal{L}$ в виде диска с тремя дырками и рассмотрим кривые $\beta_{1}, \beta_{2}, \beta_{3}$, изображенные на рис. 2. Заметим, что если вложенные окружности $\beta_{1}, \beta_{2}$ на поверхности $\Sigma$ пересекаются трансверсально в двух точках, то трубчатая окрестность $U$ графа $\beta_{1} \cup \beta_{2}$ есть диск с тремя дырками и вся конфигурация диффеоморфна изображенной на рис. 2. Будем называть такую поверхность $\mathcal{L} \subset \Sigma$ и всю конфигурацию $\left(\mathcal{L}, \alpha_{i}, \beta_{j}\right)$ фонарной конфигурацией на поверхности $\Sigma$, определяемой окружностями $\beta_{1}, \beta_{2}$. Для всякой фонарной конфигурации соответствующий фонарнъии элемент задается формулой

$$
L\left(\beta_{1}, \beta_{2}\right):=\left(T_{\alpha_{1}} T_{\alpha_{2}} T_{\alpha_{3}} T_{\alpha_{4}}\right)\left(T_{\beta_{1}} T_{\beta_{2}} T_{\beta_{3}}\right)^{-1}
$$

Цепное и фонарное соотношения - это равенства $C\left(\alpha, \beta, \beta^{\prime}\right)=1$ и $L\left(\beta_{1}, \beta_{2}\right)=1$ для соответствующих элементов. Конфигурация и соотношение называются неразбивающими, если все участвующие в них кривые неразбивающие.

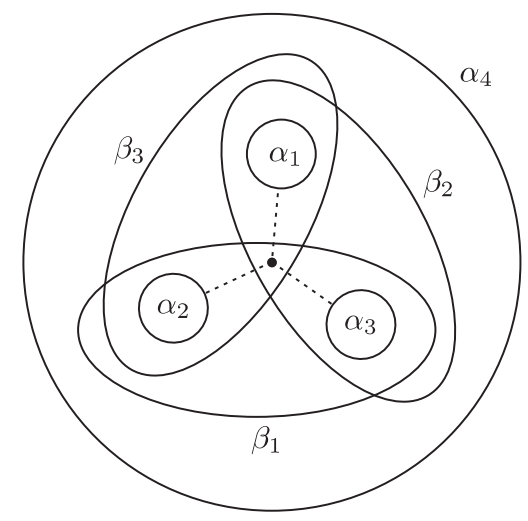

Рис. 2. Окружности в фонарной конфигурации

Как подсказывает терминология, соотношения из определения 2.17 выполнены в группе классов отображений Мap $_{g, k,[l]}$ поверхности рода $g$ с $k$ отмеченными точками и $l$ граничными окружностями. (Обозначение использует тот факт, что группа $\mathrm{Map}_{g, k,[l]}$ может быть определена как группа классов отображений поверхности $\Sigma$ рода $g$ с $k+l$ отмеченными точками, в $l$ из которых, скажем $z_{1}, \ldots, z_{l}$, задано оснащение, т. е. тривиализация касательной плоскости $T_{z_{i}} \Sigma$.) За исключением леммы 3.2 , нас будет интересовать только случай одной отмеченной точки, и мы будем использовать сокращенные обозначения Map $_{g, 1}$ или $\operatorname{Map}_{g,[1]}$, не выписывая нулевой индекс $k$ или $l$.

ОПРЕДЕЛЕНИЕ 2.18. Если $g=1$, положим $\mathcal{S}_{1}:=\mathrm{A}_{2}$, так что граф системы $\mathcal{S}_{1}$ состоит из двух вершин $s_{1}$ и $s_{2}$, соединенных ребром. Если $g \geqslant 2$, то 
система $\mathcal{S}_{g}$ определяется как расширение линейного графа Дынкина $\mathrm{A}_{2 g}$ одной вершиной $s_{0}$, соединенной ребром только с вершиной $s_{4}$. Таким образом, граф системы $\mathcal{S}_{g}$ имеет вид

$$
s_{1}-s_{2}-s_{3}-s_{4}^{s_{0}}-s_{5}-s_{6}-\ldots-s_{2 g-1}-s_{2 g}
$$

Всякий элемент $\hat{t} \in \operatorname{Br}\left(\mathcal{S}_{g}\right)$, сопряженный одной из образующих $s_{i}$, называется квазиобразующей. Множество всех квазиобразующих обозначается $\widehat{\mathcal{T}}\left(\mathcal{S}_{g}\right)$.

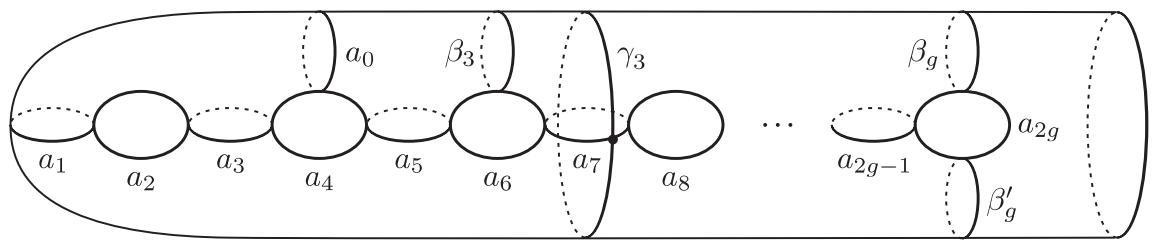

Рис. 3. Геометрические образующие группы Map $_{g,[1]}$

TEOPEма 2.19. Группа Map $_{g,[1]}$ изоморфна фактору обобщенной группы кос $\operatorname{Br}\left(\mathcal{S}_{g}\right)$ со следуюшими дополнительными соотношениями:

1) в случае $g=1$ дополнительных соотношений нет;

2) в случае $g=2$ добавляется одно неразбивающее цепное соотношение $C\left(\alpha, \beta, \beta^{\prime}\right)=1$;

3) в случае $g \geqslant 3$ добавляется одно неразбивающее иепное соотношение $C\left(\alpha, \beta, \beta^{\prime}\right)=1$ и одно неразбивающее фонарное соотношение $L\left(\beta^{\prime}, \beta^{\prime \prime}\right)=1$.

Образующие группы кос $s_{i}, i=0 ; 1, \ldots, 2 g$, могут быть реализованы как скручивания Дена относительно кривых $a_{i}$, изображенных на рис. 3. В частности, $a_{0}=\beta_{2}, a_{1}=\beta_{1}, a_{2 i}=\alpha_{i}$ для любого $i=1, \ldots, g$, а каждая кривая $a_{2 i-1}$ гомологична над $\mathbb{Z}_{2}$ сумме $\left[\beta_{i-1}\right]+\left[\beta_{i}\right]$ для любого $i=2, \ldots, g$.

Ядро гомоморфизма $\mathrm{Map}_{1,[1]} \rightarrow \mathrm{Map}_{1}$ есть центральная абелева подгруппа, порожденная элементом $\left(T_{\alpha_{1}} T_{\beta_{1}}\right)^{6}=\left(s_{1} s_{2}\right)^{6}$. B случае $g \geqslant 2$ ядро гомоморбизма $\mathrm{Map}_{g,[1]} \rightarrow \operatorname{Map}_{g}$ нормально порождается произведением $T_{\beta_{g}} T_{\beta_{g}^{\prime}}^{-1}$, где $T_{\beta_{g}}, T_{\beta_{g}^{\prime}}$ - скручивания Дена относительно кривых $\beta_{g}, \beta_{g}^{\prime}$.

Это копредставление было найдено Б. Вайнрыбом [42] (с исправлениями в [10]). М. Мацумото [35] указал простой явный способ выразить соотношения в терминах элементов Гарсайда некоторых подсистем Кокстера в системе $\mathcal{S}_{g}$.

Теорема 2.20. і) Цепное соотношение может быть задано элементом $C_{0}:=\Delta^{-4}\left(\mathrm{~A}_{4}\right) \Delta^{2}\left(\mathrm{~A}_{5}\right)$, где $\mathrm{A}_{4} u \mathrm{~A}_{5}$ - подсистемъь Кокстера $\left\{s_{2}, s_{3}, s_{4}, s_{0}\right\} u$ $\left\{s_{1}, s_{2}, s_{3}, s_{4}, s_{0}\right\}$ соответственно.

ii) Фонарное соотношение можно задать элементом $C_{0} \cdot \Delta^{-2}\left(E_{6}\right) \Delta\left(E_{7}\right)$, где $C_{0}$ - введенный выше иепной элемент, а $E_{6} u E_{7}$ - подсистемы Кокстера $\left\{s_{0}, s_{2}, \ldots, s_{6}\right\} u\left\{s_{0}, s_{1}, s_{2}, \ldots, s_{6}\right\}$ соответственно.

iii) Соотношение $T_{\beta_{g}}=T_{\beta_{g}^{\prime}}$ может быть заменено на коммутационное соотношение $\left[T_{\beta_{g}}, \Delta^{2}\left(\mathrm{~A}_{2 g}\right)\right]=1$, где $\mathrm{A}_{2 g}$ - подсистема Кокстера $\left\{s_{1}, s_{2}, \ldots, s_{2 g}\right\}$. 
Известно, что любые две неразбивающие цепные (или фонарные) конфигурации сопряжены диффеоморфизмом $f \in \mathscr{D}$ iff $f_{+}\left(\Sigma, z_{0}\right)$. Отсюда следует неявно использованное в формулировке теоремы 2.20 утверждение о том, что нормально порожденная неразбивающим цепным соотношением $C\left(\alpha, \beta, \beta^{\prime}\right)$ подгруппа не зависит от выбора цепной конфигурации, и такое же утверждение верно для фонарного соотношения. Доказательство этого утверждения и его обсуждение можно найти в работе [22].

Будем называть элемент $\Delta^{2}\left(\mathrm{~A}_{2 g}\right)$ гиперэллиптическим элементом, а соотношение $\left[T_{\beta_{g}}, \Delta^{2}\left(\mathrm{~A}_{2 g}\right)\right]=1$ (или сам коммутатор в его левой части) гиперэллиптическим соотношением. Объяснением этой терминологии служит тот факт, что элемент $\Delta^{2}\left(\mathrm{~A}_{2 g}\right) \in \operatorname{Br}\left(\mathcal{S}_{g}\right)$ представляет гиперэллиптическую инволюцию на поверхности $\Sigma$, которая может быть реализована на рис. 3 как вращение на угол $\pi$ вокруг горизонтальной оси, проходящей через цепочку кривых $a_{1}, a_{2}, \ldots, a_{2 g}$. Кроме того, будем называть соотношение $\Delta^{2}\left(\mathrm{E}_{6}\right)=\Delta\left(\mathrm{E}_{7}\right)$ модифицированным фонарным соотношением, а произведение $\Delta^{-2}\left(\mathrm{E}_{6}\right) \Delta\left(\mathrm{E}_{7}\right)$ (базиснъм ) модифицированным фонарным элементом. Те же названия будут использоваться для всех элементов, сопряженных к определенным в этом абзаце.

Все образующие $s_{i}$ сопряжены в группе $\operatorname{Br}\left(\mathcal{S}_{g}\right)$, поэтому квазиобразующие $\hat{t} \in \widehat{\mathcal{T}}\left(\mathcal{S}_{g}\right)$ являются предпочтительными подъемами скручиваний Дена относительно неразбивающих кривых $\delta$. При этом класс $\mathbb{Z}_{2}$-гомологий $[\delta] \in \mathrm{H}_{1}\left(\Sigma, \mathbb{Z}_{2}\right)$ определяется образом элемента $\hat{t}$ в группе $\mathrm{W}\left(\mathcal{S}_{g}\right)$. Поэтому мы получаем $\mathrm{W}\left(\mathcal{S}_{g}\right)$-эквивариантное отображение множеств $\mathcal{T}\left(\mathcal{S}_{g}\right) \rightarrow \mathcal{H}_{g}:=\mathrm{H}_{1}\left(\Sigma, \mathbb{Z}_{2}\right) \backslash\{0\}$. (Это отображение взаимно однозначно при $g=1,2,3$, но при $g \geqslant 4$ множество $\mathcal{T}\left(\mathcal{S}_{g}\right)$ бесконечно и наше отображение только сюръективно.) Далее, образ любой квазиобразующей $\hat{t}$ в группе $\boldsymbol{S p}\left(2 g, \mathbb{Z}_{2}\right)$ имеет порядок 2 . Отсюда следует существование гомоморфизма $\mathrm{W}\left(\mathcal{S}_{g}\right) \rightarrow \boldsymbol{S p}\left(2 g, \mathbb{Z}_{2}\right)$.

ОПРЕДЕлЕниЕ 2.21. Ядро $\mathrm{WI}_{g}:=\operatorname{Ker}\left(\mathrm{W}\left(\mathcal{S}_{g}\right) \rightarrow \boldsymbol{S p}\left(2 g, \mathbb{Z}_{2}\right)\right)$ называется группой Вейля-Торелли поверхности $\Sigma$.

Из определения 2.21 следует, что $\mathcal{H}_{g}$ - фактормножество $\mathcal{T}\left(\mathcal{S}_{g}\right) / \mathrm{W} \mathcal{I}_{g}$. Значит, группа $\mathbb{Z}\left\langle\mathcal{H}_{g}\right\rangle$ естественно изоморфна группе коинвариантов $\mathrm{P}_{\mathrm{ab}}\left(\mathcal{S}_{g}\right) \mathrm{w} \mathcal{I}_{g}$. Это позволяет применить теорему 2.14 с заменой группы $\mathbb{Z}\left\langle\mathcal{T}\left(\mathcal{S}_{g}\right)\right\rangle$ на группу $\mathbb{Z}\left\langle\mathcal{H}_{g}\right\rangle$. Кроме того, мы можем свести расширение $1 \rightarrow \mathrm{P}\left(\mathcal{S}_{g}\right) \rightarrow \operatorname{Br}\left(\mathcal{S}_{g}\right) \rightarrow$ $\mathrm{W}\left(\mathcal{S}_{g}\right) \rightarrow 1$ к расширению $1 \rightarrow \mathbb{Z}\left\langle\mathcal{H}_{g}\right\rangle \rightarrow E \rightarrow \mathrm{W}\left(\mathcal{S}_{g}\right) \rightarrow 1$. Однако в случае $g \geqslant 3$ группа $\mathbf{W}\left(\mathcal{S}_{g}\right)$ больше, чем группа $\boldsymbol{S p}\left(2 g, \mathbb{Z}_{2}\right)$, а группа Вейля-Торелли $\mathbf{W} \mathcal{I}_{g}$ порождается фонарным элементом и невидима в группе $\mathrm{Map}_{g}$. Хуже того, W $\mathcal{I}_{g}$ бесконечна при $g \geqslant 4$, что делает эффективные вычисления в группе $\mathrm{W} \mathcal{I}_{g}$ затруднительными. Для того чтобы понять, какую часть группы $W \mathcal{I}_{g}$ мы можем отфакторизовать, нам необходимо описание ядра $\operatorname{Ker}\left(\operatorname{Br}\left(\mathcal{S}_{g}\right) \rightarrow \boldsymbol{S p}\left(2 g, \mathbb{Z}_{2}\right)\right)$.

ПреДЛОЖЕНИЕ 2.22. ЯДро гомоморфизма $\operatorname{Br}\left(\mathcal{S}_{g}\right) \rightarrow \boldsymbol{S p}\left(2 g, \mathbb{Z}_{2}\right)$ порождается квадратами квазиобразующих $\hat{t}^{2}$ и модифицированными фонарными элементами $x *\left(\Delta^{-2}\left(E_{6}\right) \Delta\left(E_{7}\right)\right), x \in \operatorname{Br}\left(\mathcal{S}_{g}\right)$.

ДокАЗАТЕЛЬСтво. По лемме 2.16 любой элемент из ядра гомоморфизма $\operatorname{Br}\left(\mathcal{S}_{g}\right) \rightarrow \operatorname{Map}_{g}$ может быть представлен как произведение квадратов квазиобразующих $\hat{t}^{2}$ и фонарных элементов. Следовательно, достаточно доказать соответствующее утверждение в группе $\mathrm{Map}_{g}$. 
Обозначим через $\boldsymbol{G}$ ядро $\operatorname{Ker}\left(\operatorname{Map}_{g} \rightarrow \boldsymbol{S p}\left(2 g, \mathbb{Z}_{2}\right)\right)$ и через $\boldsymbol{G}_{0}$ подгруппу, порожденную произведениями вида $T_{\delta_{1}}^{\epsilon_{1}} T_{\delta_{2}}^{\epsilon_{2}}$, где $\epsilon_{i}= \pm 1$ и $\left[\delta_{1}\right]=\left[\delta_{2}\right] \neq 0 \in$ $\mathrm{H}_{1}\left(\Sigma, \mathbb{Z}_{2}\right)$. Будем обозначать символом "三” равенство по модулю 2 , т. е. равенство в $\mathbb{Z}_{2}, \mathrm{H}_{1}\left(\Sigma, \mathbb{Z}_{2}\right)$ или $\boldsymbol{S p}\left(2 g, \mathbb{Z}_{2}\right)$. Пусть $\alpha_{1}, \ldots, \alpha_{g} ; \beta_{1}, \ldots, \beta_{g}$ - выбранный выше геометрический базис на поверхности $\Sigma$.

Зафиксируем элемент $f \in \boldsymbol{G}$ и запишем его в виде произведения скручиваний Дена относительно неразбивающих кривых $f=\prod_{i=1}^{n} T_{\delta_{i}}^{\epsilon_{i}}$. Соотношения в группе кос $T_{\gamma} T_{\delta}^{\epsilon}=T_{\delta^{\prime}}^{\epsilon} T_{\gamma}$, где $\epsilon= \pm 1$ и $\delta^{\prime}=T_{\gamma}(\delta)$, позволяют нам использовать движения Гурвица для того, чтобы сдвигать любое конкретное скручивание Дена $T_{\gamma}$ в этом произведении в любую сторону без изменений. Заметим еще, что образующие $T_{\delta_{1}}^{\epsilon_{1}} T_{\delta_{2}}^{\epsilon_{2}}$ группы $\boldsymbol{G}_{0}$ сохраняются при сопряжении.

Шаг 1. Предположим, что существуют сомножители вида $T_{\delta_{i}}$, где $\delta_{i} \cap \beta_{1} \equiv 0$. Соберем всех их справа, используя соотношения в группе кос. Итак, будем считать, что $\delta_{i} \cap \beta_{1} \equiv 1$ при $i=1, \ldots, n_{1}$, но $\delta_{i} \cap \beta_{1} \equiv 0$ при $i=n_{1}+1, \ldots, n$.

Шаг 2. Предположим, что $\delta_{i} \cap \delta_{j} \equiv 1$ для различных $i, j \leqslant n_{1}$. Сдвинем эти сомножители вместе, так что $j=i+1$, и применим соотношение в группе кос. При этом $T_{\delta_{i}} T_{\delta_{i+1}}$ преобразуется в $T_{\gamma} T_{\delta_{i}}$, где $\gamma \equiv \delta_{i}+\delta_{i+1}$. Следовательно, $\gamma \cap \beta_{1} \equiv 0$, и мы можем сдвинуть полученное скручивание $T_{\gamma}$ вправо. Это позволяет нам уменьшить число $n_{1}$ таких $\delta_{i}$, что $\delta_{i} \cap \beta_{1} \equiv 1$. Повторяя этот шаг, мы сводим задачу к случаю, когда $\delta_{i} \cap \delta_{j} \equiv 0$ для всех $i, j \leqslant n_{1}$.

Шаг 3. Пусть $n_{1}>0$, т. е. существуют сомножители $T_{\delta_{i}}$, для которых $\delta_{i} \cap \beta_{1} \equiv 1$. Возьмем начальную часть нашего произведения $\prod_{i=1}^{n_{1}} T_{\delta_{i}}$ и умножим его слева на $T_{\beta_{1}}^{-1} T_{\beta_{1}}$. Дважды применим соотношение в группе кос к слову $T_{\beta_{1}}^{-1} T_{\beta_{1}} T_{\delta_{1}}$, т. е. выполним преобразования

$$
T_{\beta_{1}}^{-1} T_{\beta_{1}} T_{\delta_{1}} \Rightarrow T_{\beta_{1}}^{-1} T_{\delta} T_{\beta_{1}} \Rightarrow T_{\beta_{1}}^{-1} T_{\gamma} T_{\delta},
$$

где $\delta:=T_{\beta}\left(\delta_{1}\right)$ и $\gamma:=T_{\delta}\left(\beta_{1}\right)$. Тогда $\delta \equiv \delta_{1}+\beta_{1}$ и $\delta \cap \delta_{i} \equiv 1$ для всех $i=2, \ldots, n_{1}$. Сдвинем теперь $T_{\delta}$ вправо от всех $T_{\delta_{i}}, i=2, \ldots, n_{1}$, т. е. преобразуем

$$
T_{\beta_{1}}^{-1} T_{\gamma} T_{\delta} \prod_{i=2}^{n_{1}} T_{\delta_{i}} \Rightarrow T_{\beta_{1}}^{-1} T_{\gamma} \prod_{i=2}^{n_{1}} T_{\delta_{i}^{\prime}} T_{\delta},
$$

где $\delta_{i}^{\prime}:=T_{\delta}\left(\delta_{i}\right)$. Теперь $\delta_{i}^{\prime} \equiv \delta_{i}+\delta$ и $\delta_{i}^{\prime} \cap \beta_{i} \equiv 0$. Наконец, сдвинем $T_{\beta_{1}}^{-1}$ и полученные нами $T_{\delta_{i}^{\prime}}, i=2, \ldots, n_{1}$, вправо до конца. Получится новое разложение $f=\prod_{i=1}^{n+2} T_{\delta_{i}^{\prime \prime}}$ исходного элемента $f$, в котором $\delta_{i}^{\prime \prime} \cap \beta_{1} \equiv 0$ для всех $i=3, \ldots, n+2$ и $\delta_{1}^{\prime \prime} \cap \beta_{1} \equiv 1$.

Шаг 4. Если $\delta_{2}^{\prime \prime} \cap \beta_{1} \equiv 0$, то $\prod_{i=1}^{n+2} T_{\delta_{i}^{\prime \prime}}\left(\beta_{1}\right) \equiv \beta_{1}+\delta_{1}^{\prime \prime} \not \equiv \beta_{1}$, что противоречит условию $f_{*} \equiv$ id на $\mathbb{Z}_{2}$-гомологиях. Если $\delta_{1}^{\prime \prime} \cap \delta_{2}^{\prime \prime} \equiv 1$, преобразуем $T_{\delta_{1}^{\prime \prime}} T_{\delta_{2}^{\prime \prime}}$ в $T_{\delta_{2}^{\prime \prime \prime}} T_{\delta_{1}^{\prime \prime}}$, где $\delta_{2}^{\prime \prime \prime} \cap \beta_{1} \equiv 0$. Получается то же самое противоречие. Значит, $\delta_{1}^{\prime \prime} \cap \delta_{2}^{\prime \prime} \equiv 0$ и $\delta_{1}^{\prime \prime} \cap \beta_{1} \equiv \delta_{2}^{\prime \prime} \cap \beta_{1} \equiv 1$. Но тогда $\beta_{1} \equiv \prod_{i=1}^{n+2} T_{\delta_{i}^{\prime \prime}}\left(\beta_{1}\right) \equiv \beta_{1}+\delta_{1}^{\prime \prime}+\delta_{2}^{\prime \prime}$ и, следовательно, $\delta_{1}^{\prime \prime} \equiv \delta_{2}^{\prime \prime}$. Это означает, что элемент $T_{\delta_{1}^{\prime \prime}} T_{\delta_{2}^{\prime \prime}}$ лежит в группе $\boldsymbol{G}_{0}$. Сдвинем его направо. Таким способом мы представим $f$ в виде $\prod_{i} T_{\delta_{i}} \cdot f_{1}$, где $f_{1} \in \boldsymbol{G}_{0}$ и $\delta_{i} \cap \beta_{1} \equiv 0$.

Шаг 5. Повторим шаги 1-4 последовательно для кривых $\beta_{2}, \ldots, \beta_{g}$ вместо $\beta_{1}$. Для каждого $\beta_{k}$ полученные ранее соотношения $\delta_{i} \cap \beta_{j} \equiv 0$ при $i=1, \ldots, n$ и $j=1, \ldots, k-1$ останутся выполненными. В результате мы 
представим $f$ в виде $\left(\prod_{i} T_{\delta_{i}}\right) \cdot f_{2}$, где $f_{2} \in \boldsymbol{G}_{0}$ и $\delta_{i} \cap \beta_{j} \equiv 0$ для всех $j=1, \ldots, g$. Отсюда следует, что после выполнения этого шага каждый класс $\mathbb{Z}_{2}$-гомологий $\left[\delta_{i}\right]$ будет линейной комбинацией $\mathbb{Z}_{2}$-классов $\left[\beta_{1}\right], \ldots,\left[\beta_{g}\right]$, а значит, $\delta_{i} \cap \delta_{j} \equiv 0$ для всех $i, j=1, \ldots, n$. Следовательно, применение соотношений группы кос не меняет класс $\mathbb{Z}_{2}$-гомологий оставшихся кривых $\delta_{i}$ : если $T_{\delta_{i}} T_{\delta_{j}} T_{\delta_{i}}^{-1}=T_{\delta_{j}^{\prime}}$, то $\delta_{j} \equiv \delta_{j}^{\prime}$.

Шаг 6. Рассмотрим кривую $\alpha_{g}$. Пусть $\Delta_{\alpha_{g}}-$ множество тех $\delta_{i}$, для которых $\alpha_{g} \cap \delta_{i} \equiv 1$, и пусть $V_{\alpha_{g}}$ - векторное пространство над $\mathbb{Z}_{2}$, порожденное $\Delta_{\alpha_{g}}$. Предположим, что $\operatorname{dim}_{\mathbb{Z}_{2}} V_{\alpha_{g}} \geqslant 3$. Выберем три окружности в $\Delta_{\alpha_{g}}$, скажем $\delta_{1}, \delta_{2}, \delta_{3}$, классы гомологий которых линейно независимы над $\mathbb{Z}_{2}$. Тогда $\left[\delta_{2}\right] \equiv\left[\delta_{1}\right]+\left[\gamma_{2}\right]$ и $\left[\delta_{3}\right] \equiv\left[\delta_{1}\right]+\left[\gamma_{3}\right]$ для некоторых классов $\left[\gamma_{2}\right],\left[\gamma_{3}\right] \in \mathrm{H}_{1}\left(\Sigma, \mathbb{Z}_{2}\right)$, где $\left[\delta_{1}\right],\left[\gamma_{2}\right],\left[\gamma_{3}\right]$ линейно независимы над $\mathbb{Z}_{2}$. Заметим, что $\gamma_{1} \cap \alpha_{g} \equiv\left(\delta_{i}-\delta_{1}\right) \cap \alpha_{g} \equiv 0$. Реализуем классы гомологий $\left[\gamma_{2}\right],\left[\gamma_{3}\right] \in \mathrm{H}_{1}\left(\Sigma, \mathbb{Z}_{2}\right)$ вложенными кривыми $\gamma_{2}, \gamma_{3} \subset \Sigma$, не пересекающимися с $\delta_{1}$ и друг с другом. Выберем точку $z_{0} \in \Sigma$, не лежащую на $\delta_{1}, \gamma_{2}, \gamma_{3}$, и вложенные дуги $a_{1}, a_{2}, a_{3}$, соединяющие точку $z_{0}$ с кривыми $\delta_{1}, \gamma_{2}, \gamma_{3}$ соответственно и не пересекающие друг друга и кривые $\delta_{1}, \gamma_{2}, \gamma_{3}$, за исключением своих концевых точек. Тогда трубчатая окрестность $U$ графа $\Gamma$, образованного кривыми $\delta_{1}, \gamma_{2}, \gamma_{3}$ и $a_{1}, a_{2}$, $a_{3}$, есть диск с тремя дырками, ограниченными кривыми $\delta_{1}, \gamma_{2}, \gamma_{3}$, (см. рис. 2). Умножим произведение $\prod_{i=1}^{n} T_{\delta_{i}}$ на фонарное соотношение

$$
T_{\delta_{1}+\gamma_{2}}^{-1} T_{\delta_{1}+\gamma_{3}}^{-1} T_{\gamma_{2}+\gamma_{3}}^{-1} T_{\delta_{1}} T_{\gamma_{2}} T_{\gamma_{3}} T_{\delta_{1}+\gamma_{2}+\gamma_{3}}=\mathrm{id}
$$

(см. рис. 2). Сомножители $T_{\delta_{1}+\gamma_{2}}^{-1} T_{\delta_{2}}$ и $T_{\delta_{1}+\gamma_{3}}^{-1} T_{\delta_{3}}$ сокращаются. Сдвинем сомножитель $T_{\delta_{1}}^{2} \in \boldsymbol{G}_{0}$ направо. Получится произведение $\prod_{i=1}^{n+1} T_{\delta_{i}^{\prime}}$, содержащее менъше сомножителей $T_{\delta_{i}^{\prime}}$, для которых $\delta_{i}^{\prime} \cap \alpha_{g} \equiv 1$. Повторяя эту процедуру, мы сводим ситуацию к случаю $\operatorname{dim}_{\mathbb{Z}_{2}} V_{\alpha_{g}} \leqslant 2$.

Шаг 7. Сохраним обозначения $\Delta_{\alpha_{g}}$ и $V_{\alpha_{g}}$ из шага 6. Если $\operatorname{dim}_{\mathbb{Z}_{2}} V_{\alpha_{g}}=0$, то $\delta_{i} \cap \alpha_{g} \equiv 0$ для всех сомножителей $T_{\delta_{i}}$, и мы переходим к следующему шагу. Предположим, что $\operatorname{dim}_{\mathbb{Z}_{2}} V_{\alpha_{g}}=1$. Тогда $\delta_{i} \equiv \delta_{j}$ для всех $\delta_{i}, \delta_{j} \in \Delta_{\alpha_{g}}$. Следовательно, $\prod_{i=1}^{n} T_{\delta_{i}}\left(\alpha_{g}\right) \equiv \alpha_{g}+n_{\alpha_{g}} \delta_{1}$, где $\delta_{1}$ - какой-нибудь элемент множества $\Delta_{\alpha_{g}}$, а $n_{\alpha_{g}}$ - количество элементов в $\Delta_{\alpha_{g}}$. Отсюда следует, что число $n_{\alpha_{g}}$ четно, и мы можем объединить сомножители $T_{\delta_{i}}$ в пары вида $T_{\delta_{2 i-1}} T_{\delta_{2 i}}$, лежащие в группе $\boldsymbol{G}_{0}$.

Шаг 8. Пусть $\operatorname{dim}_{\mathbb{Z}_{2}} V_{\alpha_{g}}=2$. Возьмем два элемента из множества $\Delta_{\alpha_{g}}$, скажем $\delta_{1}$ и $\delta_{2}$, классы гомологий которых линейно независимы. Тогда любая кривая $\delta_{i} \in \Delta_{\alpha_{g}}$ гомологична над $\mathbb{Z}_{2}$ либо $\delta_{1}$, либо $\delta_{2}$, либо $\delta_{1}+\delta_{2}$. Найдем $\gamma_{1}, \gamma_{2} \in \mathrm{H}_{1}\left(\Sigma, \mathbb{Z}_{2}\right)$ такие, что $\delta_{1} \cap \gamma_{1} \equiv \delta_{2} \cap \gamma_{2} \equiv 1, \delta_{1} \cap \gamma_{2} \equiv \delta_{2} \cap \gamma_{1} \equiv \gamma_{1} \cap \gamma_{2} \equiv 0$ и $\delta_{i} \cap \gamma_{j} \equiv 0$ для любой $\delta_{i}$, не содержащейся в $\Delta_{\alpha_{g}}$. (Это можно сделать, выбрав подходящий $\mathbb{Z}_{2}$-базис Дарбу в пространстве $\mathrm{H}_{1}\left(\Sigma, \mathbb{Z}_{2}\right)$.) Тогда

$$
\prod_{i=1}^{n} T_{\delta_{i}}\left(\gamma_{1}\right)-\gamma_{1} \equiv \sum_{i=1}^{n}\left(\gamma_{1} \cap \delta_{i}\right) \delta_{i},
$$

и потому

$$
\gamma_{2} \cap\left(\prod_{i=1}^{n} T_{\delta_{i}}\left(\gamma_{1}\right)-\gamma_{1}\right) \equiv n_{12}
$$


где $n_{12}$ - число кривых $\delta_{i} \in \Delta_{\alpha_{g}}$ таких, что $\delta_{i} \equiv \delta_{1}+\delta_{2}$. Следовательно, число $n_{12}$ четно, и мы можем объединить в пары сомножители $T_{\delta_{i}}$, для которых $\delta_{i} \equiv \delta_{1}+\delta_{2}$, как на шаге 6 . Аналогичным образом показывается, что числа $n_{1}$ и $n_{2}$ таких $\delta_{i} \in \Delta_{\alpha_{g}}$, что $\delta_{i} \equiv \delta_{1}$ или, соответственно, $\delta_{i} \equiv \delta_{2}$, также четны. Поэтому все сомножители $T_{\delta_{i}}$, для которых $\delta_{i} \in \Delta_{\alpha_{g}}$, можно собрать в пары $T_{\delta_{2 i-1}} T_{\delta_{2 i}}$, лежащие в группе $\boldsymbol{G}_{0}$.

Шаг 9. После выполнения шага 8 оставшиеся сомножители $T_{\delta_{i}}$ удовлетворяют соотношению $\delta_{i} \cap \alpha_{g} \equiv 0$. Повторим шаги 6-8 для кривых $\alpha_{g-1}, \alpha_{g-2}, \ldots$ Заметим, что уже полученные соотношения $\mathbb{Z}_{2}$-ортогональности классам кривых $\beta_{1}, \ldots, \beta_{g} ; \alpha_{g}$ сохранятся. В итоге получится равенство $\boldsymbol{G}_{0}=\boldsymbol{G}$.

Таким образом, мы показали, что ядро $\operatorname{Ker}\left(\operatorname{Map}_{g} \rightarrow \boldsymbol{S p}\left(2 g, \mathbb{Z}_{2}\right)\right)$ порождается произведениями вида $T_{\delta_{1}}^{\epsilon_{1}} T_{\delta_{2}}^{\epsilon_{2}}$, где $\epsilon_{i}= \pm 1$ и $\left[\delta_{1}\right]=\left[\delta_{2}\right] \neq 0 \in \mathrm{H}_{1}\left(\Sigma, \mathbb{Z}_{2}\right)$.

Шаг 10. Умножая при необходимости образующую $T_{\delta_{1}}^{\epsilon_{1}} T_{\delta_{2}}^{\epsilon_{2}}$ на квадраты скручиваний $T_{\delta_{1}}^{2}$ и $T_{\delta_{2}}^{-2}$, мы можем привести ее к виду $T_{\delta_{1}} T_{\delta_{2}}^{-1}$. Обозначим $\delta_{1}=: \alpha, \delta_{2}=: \alpha^{\prime}$, так что образующая примет вид $T_{\alpha} T_{\alpha^{\prime}}^{-1}$. Перепишем полученное произведение в виде $T_{\alpha} T_{\gamma}^{-2} T_{\gamma}^{2} T_{\alpha^{\prime}}^{-1}$. Сдвинем $T_{\gamma}^{2}$ вправо, сопрягая $T_{\alpha^{\prime}}^{-1}$, а затем сдвинем $T_{\gamma}^{-2}$ вправо с сопряжением. Тогда $T_{\alpha} T_{\gamma}^{-2} T_{\gamma}^{2} T_{\alpha^{\prime}}^{-1}$ преобразуется в $T_{\alpha} T_{\alpha^{\prime \prime}}^{-1} T_{\gamma^{\prime}}^{-2} T_{\gamma}^{2}$, где $\alpha^{\prime \prime}=T_{\gamma}^{2}\left(\alpha^{\prime}\right)$. Это позволяет нам заменить класс гомологий $\left[\alpha^{\prime}\right]$ на $\left[\alpha^{\prime \prime}\right]=\left[\alpha^{\prime \prime}\right]+2\left(\alpha^{\prime} \cap \gamma\right)[\gamma]$.

Утверждается, что существует последовательность таких “движений," которая переводит целочисленный класс гомологий $\left[\alpha^{\prime}\right]$ в класс $[\alpha]$. Ясно, что достаточно найти обратное преобразование от $[\alpha]$ к $\left[\alpha^{\prime}\right]$. Выберем такую кривую $\beta$, что $\alpha \cap \beta=1$. Тогда $\left[\alpha^{\prime}\right]=(2 k+1)[\alpha]+2 l[\beta]+2 m[\gamma]$ для некоторой неразбивающей кривой $\gamma$, для которой $\gamma \cap \alpha=\gamma \cap \beta=0$, причем как $l$, так и $m$ могут быть равны нулю. Применяя $T_{\beta}^{ \pm 2}$ к $\alpha$, мы можем заменить $2 l$ на $2 l \pm 2(2 k+1)$. Итерациями этой операции мы можем заменить $l$ на такое $l^{\prime}$, что $\left|l^{\prime}\right| \leqslant|2 k+1|$. С другой стороны, мы можем взять вместо $\beta$ новую кривую $\beta^{\prime}$ в классе гомологий $[\alpha] \pm[\beta]$. В результате число $l$ останется неизменным, а $2 k+1$ заменится на $2 k+1 \pm 2 l$. Следовательно, эти две операции позволяют избавиться от $l$.

Аналогичной процедурой можно добиться того, что $m$ будет равно нулю. Действительно, если кривая $\gamma^{\prime}$ лежит в классе гомологий $[\gamma]+[\beta]$, то отображение $T_{\gamma^{\prime}}^{ \pm 2} T_{\gamma}^{\mp 2}$ переводит класс $\left[\alpha^{\prime}\right]=(2 k+1)[\alpha]+2 l[\beta]+2 m[\gamma]$ в класс $\left[\alpha^{\prime \prime}\right]=(2 k+1)[\alpha]+2 l[\beta]+2 m^{\prime}[\gamma]$, в котором $m^{\prime}=m \pm 2(2 k+1)$. С другой стороны, обозначая $T_{\gamma^{\prime}}^{ \pm 2} T_{\gamma}^{\mp 2}$ через $f$, мы имеем

$$
T_{\alpha} T_{\alpha^{\prime}}^{-1}=T_{\alpha} \cdot f^{-1} \cdot f \cdot T_{\alpha^{\prime}}^{-1}=T_{\alpha} \cdot f^{-1} \cdot T_{\alpha^{\prime \prime}} \cdot f=T_{\alpha} \cdot T_{\alpha^{\prime \prime}} \cdot\left(f^{\prime}\right)^{-1} \cdot f,
$$

где $f^{\prime}=T_{\alpha^{\prime \prime}}^{-1} \cdot f \cdot T_{\alpha^{\prime \prime}}$. Заметим теперь, что $f$ и $f^{\prime}$ лежат в $\boldsymbol{G}$. Поэтому мы можем заменить $m$ на такое $m^{\prime}$, что $\left|m^{\prime}\right| \leqslant|2 k+1|$. Однако равенство $m^{\prime}=$ $\pm(2 k+1)$ невозможно, поскольку класс гомологий $\left[\alpha^{\prime}\right]$ примитивен. Заначит, мы получим строгое неравенство $\left|m^{\prime}\right|<|2 k+1|$. Для того чтобы изменить $k$, выберем вложенную кривую $\delta$ так, что $\delta \cap \gamma=1$ и $\delta \cap \alpha=\delta \cap \beta=0$, а затем выберем кривую $\delta^{\prime}$ в классе гомологий $[\delta]+[\alpha]$. Тогда отображение $T_{\delta^{\prime}}^{ \pm 2}$ переводит класс $(2 k+1)[\alpha]+2 m[\gamma]$ в класс $(2 k+1 \pm 4 m)[\alpha]+2 m([\gamma] \mp 2[\delta])$. Значит, мы можем сделать $|2 k+1|$ меньшим, чем $2 m$, возможно, изменив класс $[\gamma]$. Эта процедура закончится при $l=m=0$ и $2 k+1= \pm 1$. Поскольку скручивание 
Дена $T_{\delta}$ не зависит от выбора ориентации на кривой $\delta$, мы получаем равенство целочисленных классов гомологий $[\alpha]=\left[\alpha^{\prime}\right]$.

Шаг 11. Пусть $\alpha, \alpha^{\prime}$ - полученные выше простые кривые, $[\alpha]=\left[\alpha^{\prime}\right] \in$ $\mathrm{H}_{1}(\Sigma, \mathbb{Z})$. Тогда $\alpha^{\prime}=F_{1}(\alpha)$ для некоторого отображения $F_{1} \in \mathrm{Map}_{g}$. Как и выше, пусть $\beta$ - такая кривая, что $\alpha \cap \beta=1$. Положим $\beta^{\prime}:=F_{1}(\beta)$. Тогда $\beta^{\prime} \cap \alpha=1$ и, следовательно, $\left[\beta^{\prime}\right]=[\beta]+l[\alpha]+m[\gamma]$ в группе $\mathrm{H}_{1}(\Sigma, \mathbb{Z})$ для некоторого примитивного класса $[\gamma] \in \mathrm{H}_{1}(\Sigma, \mathbb{Z})$ такого, что $\gamma \cap \alpha=\gamma \cap \beta=0$. Значит, $T_{\alpha^{\prime}}^{-l}$ сохраняет кривую $\alpha^{\prime}$ (с точностью до изотопии), и $\left[T_{\alpha^{\prime}}^{-l}\left(\beta^{\prime}\right)\right]=[\beta]+m[\gamma]$. Далее, найдем кривую $\gamma^{\prime}$ в гомологическом классе $[\alpha]+[\gamma]=\left[\alpha^{\prime}\right]+[\gamma]$, не пересекающуюся с $\alpha^{\prime}$. Тогда $T_{\alpha^{\prime}}^{l} T_{\gamma^{\prime}}^{-l}$ сохраняет кривую $\alpha^{\prime}$ (с точностью до изотопии) и переводит класс $[\beta]+m[\gamma]$ в класс $[\beta]$.

Таким образом мы построили отображение $F_{2} \in \mathrm{Map}_{g}$, переводящее кривую $\alpha$ в $\alpha^{\prime}$ и сохраняющее целочисленный класс гомологий $[\beta]$. Заметим, что действие $F_{2}$ на $\cap$-ортогональном дополнении к подпространству $\mathbb{Z}\langle[\alpha],[\beta]\rangle$ может быть реализовано как произведение скручиваний Дена относительно кривых, не пересекающихся с $\alpha^{\prime}=F_{2}(\alpha)$ и $\beta^{\prime}:=F_{2}(\beta)$. Изменяя $F_{2}$ соответствующим образом, мы получаем отображение $F$, лежащее в группе Торелли $\mathcal{I}_{g}:=\operatorname{Ker}\left(\operatorname{Map}_{g} \rightarrow \boldsymbol{S p}(2 g, \mathbb{Z})\right)$ и такое, что $\alpha^{\prime}=F(\alpha)$.

Шаг 12. Воспользуемся теперь явным описанием группы Торелли. Известно, что группа $\mathcal{I}_{2}$ порождается скручиваниями Дена $T_{\delta}$ относительно разбивающux кривых (см. [40], а также [30]). Любая такая кривая делит поверхность $\Sigma$ на две части $\Sigma^{\prime}$ и $\Sigma^{\prime \prime}$, каждая из которых является тором с дыркой. Скручивание $T_{\delta}$ равно $\left(T_{\alpha} T_{\beta}\right)^{6}$ для любого геометрического базиса $\alpha$ и $\beta$ на $\Sigma^{\prime}$ (т. е. простых кривых на $\Sigma^{\prime}$, пересекающихся трансверсально в единственной точке). Но тогда $T_{\alpha}$ и $T_{\beta}$ сопряжены подсистеме Кокстера $\mathrm{A}_{2}:=\left\{s_{1}, s_{2}\right\}$, и потому элемент $\left(T_{\alpha} T_{\beta}\right)^{6}$ сопряжен $\Delta^{4}\left(\mathrm{~A}_{2}\right)$ и является произведением квадратов квазиобразующих $\hat{t}^{2}$.

Согласно [29] (см. также [30]) при $g \geqslant 3$ группа Торелли $\mathcal{I}_{g}$ порождена произведениями вида $T_{\eta} T_{\eta^{\prime}}^{-1}$, где $\eta, \eta^{\prime}$ - такие непересекающиеся неразбивающие кривые, что их объединение $\eta \sqcup \eta^{\prime}$ делит поверхность $\Sigma$ на две части. Обозначим эти части через $\Sigma^{\prime}$ и $\Sigma^{\prime \prime}$. Каждая из них является поверхностью с двумя граничными окружностями, а их рода $g^{\prime}$ и $g^{\prime \prime}$ связаны соотношением $g^{\prime}+g^{\prime \prime}=g-1$. Если $g^{\prime}=0$ или $g^{\prime \prime}=0$, то кривые $\eta, \eta^{\prime}$ изотопны, а произведение $T_{\eta} T_{\eta^{\prime}}^{-1}$ тривиально. Отсюда следует, что произведения $T_{\eta} T_{\eta^{\prime}}^{-1}$ с дополнительным условием $g^{\prime}=1$ также порождают группу Торелли $\mathcal{I}_{g}$. Однако в случае $g^{\prime}=1$ поверхность $\Sigma^{\prime}$ является цепной поверхностью (см. рис. 1). Используя определение цепного соотношения и конфигурацию кривых, представленных на рис. 1, мы получаем равенства

$$
T_{\eta} T_{\eta^{\prime}}^{-1}=T_{\eta}^{2} T_{\eta}^{-1} T_{\eta^{\prime}}^{-1}=T_{\eta}^{2}\left(T_{\beta} T_{\alpha} T_{\beta^{\prime}}\right)^{-4} .
$$

Заметим теперь, что цепная конфигурация $\left\{\beta, \alpha, \beta^{\prime}\right\}$ сопряжена цепной конфигурации $\left\{a_{1}, a_{2}, a_{3}\right\}=: \mathrm{A}_{3}$, представленной на рис. 3. Следовательно, элемент $\left(T_{\beta} T_{\alpha} T_{\beta^{\prime}}\right)^{4}$ сопряжен элементу Гарсайда $\Delta^{2}\left(\mathrm{~A}_{3}\right)$ и может быть представлен в виде произведения квадратов квазиобразующих $\hat{t}^{2}$.

СледСтвие 2.23. і) Ядро $\operatorname{Ker}\left(\operatorname{Map}_{g, 1} \rightarrow \boldsymbol{S p}\left(2 g, \mathbb{Z}_{2}\right)\right)$ порождается квадратами $T_{\delta}^{2}$ скручиваний Дена относительно неразбивающих кривых $\delta \subset \Sigma \backslash\left\{z_{0}\right\}$. 
ii) Группа Вейля-Торелли $\mathrm{WI}_{g}=\operatorname{Ker}\left(\mathrm{W}\left(\mathcal{S}_{g}\right) \rightarrow \boldsymbol{S p}\left(2 g, \mathbb{Z}_{2}\right)\right)$ порождается элементами, сопряженными элементу $w_{\circ}\left(E_{7}\right)$.

Предложение 1.9 сводит доказательство топологического утверждения о существовании или несуществовании специальных вложений бутылки Клейна в топологические семейства Лефшеца к проверке некоторых алгебраических соотношений в группе классов отображений $\mathrm{Map}_{g}$. Оказывается, что эти соотношения выполняются в группе $\boldsymbol{S} \boldsymbol{p}\left(2 g, \mathbb{Z}_{2}\right)$. Подняв соответствующие элементы в обобщенную группу кос $\operatorname{Br}\left(\mathcal{S}_{g}\right)$, мы можем затем спроектировать их в $\mathbb{Z}\left\langle\mathcal{H}_{g}\right\rangle$. Поэтому нам необходимы средства, позволяющие изучить комбинаторную структуру групп $\mathcal{H}_{g}$ и $\mathbb{Z}\left\langle\mathcal{H}_{g}\right\rangle$.

Опишем первое такое средство. Пусть $R$ - коммутативное кольцо, а $H$ - свободный $R$-модуль конечного ранга. Обозначим через $\mathrm{T}^{\bullet} H$ тензорную алгебру $H$ над $R$. Для каждой степени $d$ пусть $\mathrm{T}_{\text {Sym }}^{d} H \subset \mathrm{T}^{d} H-$ подмодуль тензоров, инвариантных относительно естественного действия симметрической группы $\mathrm{Sym}_{d}$, переставляющей тензорные сомножители $H \otimes \cdots \otimes H$. Введем •-умножение на $\mathrm{T}^{\bullet} H$ следующим образом. Для $A \in \mathrm{T}^{k} H$ и $B \in \mathrm{T}^{l} H$ положим

$$
A \bullet B:=\sum_{\sigma \in \operatorname{Sym}_{k+l}}^{\prime} \sigma(A \otimes B),
$$

где сумма $\sum^{\prime}$ берется по всем перестановкам $\sigma \in$ Sym $_{k+l}$ тензорных сомножителей, сохраняющим порядок первых $k$ и последних $l$ сомножителей. Другими словами, $\sigma \in \mathrm{Sym}_{k+l}$ удовлетворяет условию $\sigma(i)<\sigma(j)$, если $i<j \leqslant k$ и если $k<i<j$. В частности,

$$
v \bullet w=v \otimes w+w \otimes v, \quad u \bullet(v \otimes w)=u \otimes v \otimes w+v \otimes u \otimes w+v \otimes w \otimes u
$$

для $u, v, w \in H$. Непосредственно проверяются следующие свойства этих структур:

1) $v^{\otimes d} \in \mathrm{T}_{\text {ธут }}^{d} H$ для любого $v \in H ; A \bullet B \in \mathrm{T}_{\text {Sym }}^{k+l} H$, если $A \in \mathrm{T}_{\text {Sym }}^{k} H$ и $B \in \mathrm{T}_{\mathrm{Sym}}^{l} H$

2) •-умножение ассоциативно, коммутативно и дистрибутивно;

3) имеет место биномиальная формула

$$
(v+w)^{\otimes d}=v^{\otimes d}+v^{\otimes(d-1)} \bullet w+v^{\otimes(d-2)} \bullet w^{\otimes 2}+\cdots+w^{\otimes d}
$$

для любых $v, w \in H$;

4) $v^{\otimes k} \bullet v^{\otimes l}=\left(\begin{array}{c}k+l \\ k\end{array}\right) v^{\otimes(k+l)}$ для любого $v \in H$.

Последнее свойство показывает, что в случае свободного модуля $H \cong R^{\oplus r}$ ранга $r$, алгебра $\left(\mathrm{T}_{\text {Sуm }}^{\bullet} H, \bullet\right)$ изоморфна $r$-й тензорной степени $\mathbf{A}^{\bullet} \otimes \cdots \otimes \mathbf{A}^{\bullet}$ так называемой алгебры разделенных степеней $\mathbf{A}^{\bullet}$.

В интересующей нас ситуации $R:=\mathbb{Z}_{2}$ и $H:=\mathrm{H}_{1}\left(\Sigma, \mathbb{Z}_{2}\right)$. Определим гомоморфизмы $\wp^{d}: \mathbb{Z}_{2}\left\langle\mathcal{H}_{g}\right\rangle \rightarrow \mathrm{T}^{d} H$ формулой $\wp^{d}\left(A_{v}\right):=v^{\otimes d}$ для любого $v \in \mathcal{H}_{g}$ и будем использовать то же обозначение $\wp^{d}: \mathrm{P}\left(\mathcal{S}_{g}\right) \rightarrow \mathrm{T}^{d} H$ для сквозного отображения. Ясно, что $\wp^{d}$ принимает значения в $\mathrm{T}_{\text {Sym }}^{d} H \cong \operatorname{Sym}^{d}(H)$. Смысл гомоморфизма $\wp^{1}$ очевиден: он переводит каждый элемент $A_{v}$ в вектор $v \in \mathrm{H}_{1}\left(\Sigma, \mathbb{Z}_{2}\right)$. Для описания $\wp^{2}$ заметим, что пространство $\operatorname{Sym}^{2}(H)$ естественно изоморфно $\mathbb{Z}_{2}$-алгебре Ли $\mathfrak{s p}\left(2 g, \mathbb{Z}_{2}\right)$. В явном виде изоморфизм задается формулой 
$M \in \operatorname{Sym}^{d}(H) \leftrightarrow J \cdot M \in \mathfrak{s p}\left(2 g, \mathbb{Z}_{2}\right)$, где $J-$ это "симплектическая" матрица, $J:=\left(\begin{array}{cc}0 & \text { id } \\ \text { id } & 0\end{array}\right)$, с квадратными блоками размера $g \times g$, а $\mathfrak{s p}\left(2 g, \mathbb{Z}_{2}\right)$ реализована как матричная подалгебра Ли в $\operatorname{Mat}\left(2 g, \mathbb{Z}_{2}\right)$ относительно симплектического базиса $\alpha_{1}, \ldots, \alpha_{g} ; \beta_{1}, \ldots, \beta_{g}$ пространства $H$. Далее, группа $\mathfrak{s p}\left(2 g, \mathbb{Z}_{2}\right)$ имеет естественное расширение

$$
1 \rightarrow \mathfrak{s p}\left(2 g, \mathbb{Z}_{2}\right) \rightarrow \boldsymbol{S p}\left(2 g, \mathbb{Z}_{4}\right) \rightarrow \boldsymbol{S p}\left(2 g, \mathbb{Z}_{2}\right) \rightarrow 1,
$$

и явное вычисление с любым $T_{\delta}^{2}$ показывает, что гомоморфизм $J \cdot \wp^{2}: \mathrm{P}\left(\mathcal{S}_{g}\right) \rightarrow$ $\mathfrak{s p}\left(2 g, \mathbb{Z}_{2}\right) \hookrightarrow \boldsymbol{S} \boldsymbol{p}\left(2 g, \mathbb{Z}_{4}\right)$ совпадает с композицией отображений

$$
\mathrm{P}\left(\mathcal{S}_{g}\right) \hookrightarrow \operatorname{Br}\left(\mathcal{S}_{g}\right) \rightarrow \operatorname{Map}_{g} \rightarrow \boldsymbol{S p}(2 g, \mathbb{Z}) \rightarrow \boldsymbol{S p}\left(2 g, \mathbb{Z}_{4}\right) .
$$

Заметим, что матрицу $J$ можно записать в виде $\sum_{i=1}^{g} \alpha_{i} \bullet \beta_{i}$ для любого симплектического базиса $\alpha_{1}, \ldots, \alpha_{g} ; \beta_{1}, \ldots, \beta_{g}$ пространства $H$. Кроме того, напомним определение гиперэллиптического соотношения $\left[T_{\beta_{g}}, \Delta^{2}\left(\mathrm{~A}_{2 g}\right)\right]$ из теоремы 2.20.

Лемма 2.24. Справедливъ тождества:

i) $\wp^{3}\left(\Delta^{2}\left(\mathrm{~A}_{5}\right)\right)=\wp^{3}\left(\Delta^{2}\left(E_{7}\right)\right)=0$;

ii) $\wp^{3}\left(\left[T_{\beta_{g}}, \Delta^{2}\left(\mathrm{~A}_{2 g}\right)\right]\right)=\beta_{g} \bullet\left(\sum_{i=1}^{g} \alpha_{i} \bullet \beta_{i}\right)$.

ДоказАтельство. і) Имеют место равенства $\mathcal{T}\left(\mathrm{A}_{5}\right)=\mathcal{H}_{2}$ и $\mathcal{T}\left(\mathrm{E}_{7}\right)=\mathcal{H}_{3}$. Поэтому в обоих случаях элемент $\Delta^{2}(\cdot)$ - это сумма $\sum_{v \neq 0 \in V} A_{v}$, где подпространство $V \subset \mathrm{H}_{1}\left(\Sigma, \mathbb{Z}_{2}\right)$ имеет размерность 4 или 6 соответственно. Следовательно, $\wp^{3}\left(\Delta^{2}(\cdot)\right)$ есть сумма $\sum_{v \in V} v^{\otimes 3}$. Выберем базис $e_{1}, \ldots, e_{r}$ в подпространстве $V$ (где $r=4$ или $r=6$ в зависимости от рассматриваемого случая) и, разложив сумму $\sum_{v \in V} v^{\otimes 3}$ с помощью биномиальной формулы $(2.7)$, получим сумму мономов $e^{\mathbf{n}}:=e_{1}^{\otimes n_{1}} \bullet \cdots \bullet e_{r}^{\otimes n_{r}}$, где $n_{1}+\cdots+n_{r}=3$. Поскольку как минимум одно из чисел $n_{i}$ равно нулю, каждый моном $e^{\mathbf{n}}$ встречается в этой сумме четное число раз, и сумма равна нулю.

ii) Для краткости будем использовать обозначения $a_{i}, \alpha_{j}, \beta_{k} \in \mathcal{H}_{g}$ для классов $\mathbb{Z}_{2}$-гомологий соответствующих кривых. Положим $v_{i j}:=\sum_{k=i}^{j} a_{k}$ и $v_{i}:=v_{i, 2 g}$. Тогда образ квадрата элемента Гарсайда $\Delta^{2}\left(\mathrm{~A}_{2 g}\right)$ в $\mathbb{Z}_{2}\left\langle\mathcal{H}_{g}\right\rangle$ есть сумма $\sum_{1 \leqslant i \leqslant j \leqslant 2 g} A_{v_{i j}}$. Поскольку кривая $\beta_{g}$ не пересекается с кривой $a_{j}$ при $j<2 g$, гиперэллиптическое соотношение $\left[T_{\beta_{g}}, \Delta^{2}\left(\mathrm{~A}_{2 g}\right)\right]$ отображается в сумму $\sum_{i=1}^{2 g}\left(A_{v_{i}}+A_{v_{i}+\beta_{g}}\right)$. Поэтому

$$
\wp^{3}\left(\left[T_{\beta_{g}}, \Delta^{2}\left(\mathrm{~A}_{2 g}\right)\right]\right)=\sum_{i=1}^{2 g}\left(v_{i}^{\otimes 3}+\left(v_{i}+\beta_{g}\right)^{\otimes 3}\right) .
$$

Раскрывая скобки с учетом того, что $2=0 \in \mathbb{Z}_{2}$, мы получаем

$$
\wp^{3}\left(\left[T_{\beta_{g}}, \Delta^{2}\left(\mathrm{~A}_{2 g}\right)\right]\right)=\sum_{i=1}^{2 g}\left(v_{i} \bullet \beta_{g}^{\otimes 2}+v_{i}^{\otimes 2} \bullet \beta_{g}\right) .
$$

Подставляя выражения $v_{i}=a_{i}+a_{i+1}+\cdots+a_{2 g}$ в сумму $\sum_{i=1}^{2 g} v_{i}$, мы видим, что каждый класс $a_{j}$ участвует в ней $j$ раз. Таким образом,

$$
\sum_{i=1}^{2 g} v_{i}=a_{1}+a_{3}+\cdots+a_{2 g-1}=\beta_{1}+\left(\beta_{1}+\beta_{2}\right)+\cdots+\left(\beta_{g-1}+\beta_{g}\right)=\beta_{g} .
$$


Аналогично, подставляя $v_{i}^{\otimes 2}=\sum_{j=i}^{2 g} a_{j}^{\otimes 2}+\sum_{i \leqslant j<k \leqslant 2 g} a_{j} \bullet a_{k}$ в сумму $\sum_{i=1}^{2 g} v_{i}^{\otimes 2}$, мы получаем

$$
\sum_{i=1}^{2 g} v_{i}^{\otimes 2}=\sum_{j=1}^{2 g} j a_{j}^{\otimes 2}+\sum_{1 \leqslant j<k \leqslant 2 g} j a_{j} \bullet a_{k} .
$$

Первая сумма дает

$$
\begin{aligned}
a_{1}^{\otimes 2}+a_{3}^{\otimes 2}+\cdots+a_{2 g-1}^{\otimes 2} & =\beta_{1}^{\otimes 2}+\left(\beta_{1}+\beta_{2}\right)^{\otimes 2}+\cdots+\left(\beta_{g-1}+\beta_{g}\right)^{\otimes 2} \\
& =\beta_{1} \bullet \beta_{2}+\beta_{2} \bullet \beta_{3}+\cdots+\beta_{g-1} \bullet \beta_{g}+\beta_{g}^{\otimes 2} .
\end{aligned}
$$

Вторая сумма переписывается в виде

$$
\sum_{1 \leqslant j<k \leqslant 2 g} j a_{j} \bullet a_{k}=\sum_{j=1}^{g} \sum_{k=2 j}^{2 g} a_{2 j-1} \bullet a_{k},
$$

где внутренняя сумма $\sum_{k=2 j}^{2 g} a_{k}$ равна $\left(\alpha_{j}+\cdots+\alpha_{g}\right)+\left(\beta_{j}+\beta_{g}\right)$. Поэтому, положив $\beta_{0}:=0$, мы можем представить вторую сумму в виде

$$
\sum_{1 \leqslant j<k \leqslant 2 g} j a_{j} \bullet a_{k}=\sum_{j=1}^{g}\left(\beta_{j-1}+\beta_{j}\right) \bullet\left(\left(\alpha_{j}+\cdots+\alpha_{g}\right)+\left(\beta_{j}+\beta_{g}\right)\right) .
$$

Проведем анализ отдельных членов в правой сумме последнего равенства. Во-первых, $\sum_{j=1}^{g}\left(\beta_{j-1}+\beta_{j}\right) \bullet \beta_{g}=\beta_{g} \bullet \beta_{g}=2 \beta_{g}^{\otimes 2}=0$. Аналогично, $\beta_{j} \bullet \beta_{j}=0$ и $\sum_{j=1}^{g}\left(\beta_{j-1}+\beta_{j}\right) \bullet \beta_{j}=\beta_{1} \bullet \beta_{2}+\beta_{2} \bullet \beta_{3}+\cdots+\beta_{g-1} \bullet \beta_{g}$. Члены, содержащие данный класс $\alpha_{k}$, имеют вид

$$
\sum_{j=1}^{k}\left(\beta_{j-1}+\beta_{j}\right) \bullet \alpha_{k}=\left(\beta_{0}+\beta_{k}\right) \bullet \alpha_{k}=\beta_{k} \bullet \alpha_{k} .
$$

Наконец, мы можем вычислить

$$
\wp^{3}\left(\left[T_{\beta_{g}}, \Delta^{2}\left(\mathrm{~A}_{2 g}\right)\right]\right)=\beta_{g} \bullet \beta_{g}^{\otimes 2}+\left(\beta_{g}^{\otimes 2}+\sum_{j=1}^{g} \beta_{j} \bullet \alpha_{j}\right) \bullet \beta_{g}=\beta_{g} \bullet \sum_{j=1}^{g} \alpha_{j} \bullet \beta_{j},
$$

что и требовалось получить.

СлЕДСТВИЕ 2.25. При $g \geqslant 2$ гомоморфизм $\wp^{3}$ продолжается на группу $\operatorname{Ker}\left(\operatorname{Map}_{g, 1} \rightarrow \boldsymbol{S p}\left(2 g, \mathbb{Z}_{2}\right)\right)$ таким образом, что композиция $\wp^{3} \circ \partial_{\pi}: \pi_{1}(\Sigma) \rightarrow \mathrm{T}^{3}$ равна композиции проекции $\pi_{1}(\Sigma) \rightarrow \mathrm{H}_{1}\left(\Sigma, \mathbb{Z}_{2}\right)$ и вложения $\mathrm{H}_{1}\left(\Sigma, \mathbb{Z}_{2}\right) \hookrightarrow \mathrm{T}^{3}$, задаваемого формулой $\gamma \mapsto \gamma \bullet \sum_{i=1}^{g} \alpha_{i} \bullet \beta_{i}$.

ДокАзАтельство. Применяем следствие 2.23, i).

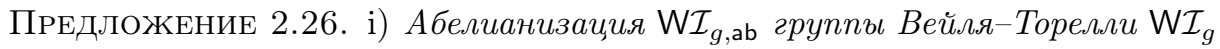
есть векторное пространство над $\mathbb{Z}_{2}$, естественно изоморфное $\wedge^{6} \mathrm{H}_{1}\left(\Sigma, \mathbb{Z}_{2}\right)$.

ii) Существует естественное расширение

$$
0 \rightarrow \mathbb{Z}\left\langle\mathcal{H}_{g}\right\rangle \rightarrow \Lambda_{g} \rightarrow \mathrm{W} \mathcal{I}_{g, \text { ab }} \rightarrow 0
$$


в котором группа $\Lambda_{g}$ может быть реализована как подрешетка в $\frac{1}{2} \mathbb{Z}\left\langle\mathcal{H}_{g}\right\rangle$, a образ индуцированного вложения $\mathrm{W} \mathcal{I}_{g, \text { ab }} \subset \frac{1}{2} \mathbb{Z}\left\langle\mathcal{H}_{g}\right\rangle / \mathbb{Z}\left\langle\mathcal{H}_{g}\right\rangle \cong \mathbb{Z}_{2}\left\langle\mathcal{H}_{g}\right\rangle$ порождается суммами вида $L_{V}:=\sum_{v \neq 0 \in V} A_{v}$, где $V$ - симплектическое шестимерное подпространство в $\mathrm{H}_{1}\left(\Sigma, \mathbb{Z}_{2}\right)$.

iii) Существуют расширение групп

$$
0 \rightarrow \Lambda_{g} \rightarrow \widehat{\boldsymbol{S p}}\left(2 g, \mathbb{Z}_{2}\right) \rightarrow \boldsymbol{S p}\left(2 g, \mathbb{Z}_{2}\right) \rightarrow 1
$$

и гомоморфизм из расширения $1 \rightarrow \mathrm{P}\left(\mathcal{S}_{g}\right) \rightarrow \operatorname{Br}\left(\mathcal{S}_{g}\right) \rightarrow \mathrm{W}\left(\mathcal{S}_{g}\right) \rightarrow 1$ на это расширение, для которого гомоморфизм $\mathrm{W}\left(\mathcal{S}_{g}\right) \rightarrow \boldsymbol{S p}\left(2 g, \mathbb{Z}_{2}\right)$ имеет обычный смисл, а гомоморфизм $\mathrm{P}\left(\mathcal{S}_{g}\right) \rightarrow \Lambda_{g}$ есть композиция $\mathrm{P}\left(\mathcal{S}_{g}\right) \rightarrow \mathrm{P}_{\mathrm{ab}}\left(\mathcal{S}_{g}\right) \rightarrow$ $\mathbb{Z}\left\langle\mathcal{H}_{g}\right\rangle \subset \Lambda_{g}$

Симплектичность подпространства $V \subset \mathrm{H}_{1}\left(\Sigma, \mathbb{Z}_{2}\right)$ означает, что ограничение формы пересечения на $V$ невырождено.

ДоКАЗАТЕЛЬСТво ПРЕДЛОЖЕНИЯ 2.26. Пусть $L_{0}:=C \cdot \Delta^{-2}\left(\mathrm{E}_{6}\right) \Delta\left(\mathrm{E}_{7}\right)$ и $L_{0}^{\prime}:=\Delta^{-2}\left(\mathrm{E}_{6}\right) \Delta\left(\mathrm{E}_{7}\right)$ - “базисные" элементы, соответствующие немодифицированному и модифицированному фонарному соотношению. Их образы в группе $\mathrm{W}\left(\mathcal{S}_{g}\right)$ равны между собой и совпадают с элементом наибольшей длины $w_{\circ}\left(\mathrm{E}_{7}\right)$ в подгруппе Кокстера $\mathrm{W}\left(\mathrm{E}_{7}\right) \subset \mathrm{W}\left(\mathcal{S}_{g}\right)$. Поскольку элемент $w_{\circ}\left(\mathrm{E}_{7}\right)$ имеет порядок 2 и нормально порождает группу $\mathbf{W} \mathcal{I}_{g}$, ее абелианизация $\mathbf{W} \mathcal{I}_{g, \text { ab }}$ является векторным пространством над $\mathbb{Z}_{2}$.

Теперь рассмотрим естественное расширение

$$
0 \rightarrow \mathbb{Z}\left\langle\mathcal{H}_{g}\right\rangle \rightarrow Q \rightarrow \mathrm{W} \mathcal{I}_{g} \rightarrow 1
$$

в котором $Q$ - это фактор ядра $\operatorname{Ker}\left(\operatorname{Br}\left(\mathcal{S}_{g}\right) \rightarrow \boldsymbol{S p}\left(2 g, \mathbb{Z}_{2}\right)\right)$ по ядру $\operatorname{Ker}\left(\mathrm{P}\left(\mathcal{S}_{g}\right) \rightarrow\right.$ $\left.\mathbb{Z}\left\langle\mathcal{H}_{g}\right\rangle\right)$. Каждый элемент в группе $Q$ представляется произведением элементов вида $x * L_{0}$, где $x \in \operatorname{Br}\left(\mathcal{S}_{g}\right)$, и элементов группы $\mathbb{Z}\left\langle\mathcal{H}_{g}\right\rangle$. Поскольку $\mathbb{Z}\left\langle\mathcal{H}_{g}\right\rangle$ содержится в центре группы $Q$, а цепное соотношение $C=L_{0}\left(L_{0}^{\prime}\right)^{-1}$ лежит в $\mathbb{Z}\left\langle\mathcal{H}_{g}\right\rangle$, коммутаторы $\left[x * L_{0}, y * L_{0}\right]$ и $\left[x * L_{0}^{\prime}, y * L_{0}\right]$ (и так далее) равны и порождают коммутант $Q^{\prime}:=[Q, Q]$.

Мы утверждаем, что пересечение $Q^{\prime} \cap \mathbb{Z}\left\langle\mathcal{H}_{g}\right\rangle$ тривиально. Пусть $z \in$ $Q^{\prime} \cap \mathbb{Z}\left\langle\mathcal{H}_{g}\right\rangle$. Условие $z \in Q^{\prime}$ означает, что $z$ представляется элементом вида $\hat{z}=\prod_{j}\left[x_{j} * L_{0}, y_{j} * L_{0}\right]$ из группы $\operatorname{Br}\left(\mathcal{S}_{g}\right)$. Поскольку элементы $x_{j} * L_{0}, y_{j} * L_{0}$ отображаются в единицу id $\in \boldsymbol{S} \boldsymbol{p}\left(2 g, \mathbb{Z}_{2}\right)$, мы можем применить к ним алгоритм из доказательства предложения 2.22. Однако теперь мы выполняем только те шаги, на которых не использовалось фонарное соотношение, т. е. не делаем шаги 6-9. В результате каждый элемент $x_{j} * L_{0}, y_{j} * L_{0}$ представляется в виде произведения элементов из $\mathbb{Z}\left\langle\mathcal{H}_{g}\right\rangle$ и скручиваний Дена $T_{\delta_{i}}$, у которых класс $\mathbb{Z}_{2}$-гомологий $\left[\delta_{i}\right]$ является линейной комбинацией классов $\left[\beta_{1}\right], \ldots,\left[\beta_{g}\right]$. Поскольку $\mathbb{Z}\left\langle\mathcal{H}_{g}\right\rangle$ лежит в центре, отсюда следует, что коммутаторы $\left[x_{j} * L_{0}, y_{j} * L_{0}\right]$ есть произведения скручиваний Дена $T_{\delta_{i}}$, у которых $\left[\delta_{i}\right] \in \mathbb{Z}_{2}\left\langle\left[\beta_{1}\right], \ldots,\left[\beta_{g}\right]\right\rangle$.

Теперь рассмотрим $z$ как элемент группы $\mathrm{P}\left(\mathcal{S}_{g}\right)$. Запишем образ элемента $z$ в группе $\mathbb{Z}\langle\mathcal{H}\rangle$ в виде $z=\sum_{v \in \mathcal{H}_{g}} n_{v} A_{v}$, где $n_{v} \in \mathbb{Z}$. Пусть $G$ - подгруппа в $\mathrm{W}\left(\mathcal{S}_{g}\right)$, полученная из $\mathrm{W} \mathcal{I}_{g}$ добавлением квазиотражений $t \in \mathcal{T}\left(\mathcal{S}_{g}\right)$, проекции которых в $\boldsymbol{S} \boldsymbol{p}\left(2 g, \mathbb{Z}_{2}\right)$ суть скручивания Дена $T_{\delta_{i}},\left[\delta_{i}\right] \in \mathbb{Z}_{2}\left\langle\left[\beta_{1}\right], \ldots,\left[\beta_{g}\right]\right\rangle$. 
Поскольку $G$ содержит $\mathrm{WI}_{g}$, существует $G$-эквивариантная проекция $\mathcal{G}_{g}=$ $\mathcal{T}\left(\mathcal{S}_{g}\right) / \mathrm{W} \mathcal{I}_{g} \rightarrow \mathcal{T}\left(\mathcal{S}_{g}\right) / G$, при которой смежные классы в $\mathcal{T}\left(\mathcal{S}_{g}\right) / G$ совпадают с $G$-орбитами в $\mathcal{G}_{g}$. Очевидно, все классы $[\delta] \in \mathbb{Z}_{2}\left\langle\left[\beta_{1}\right], \ldots,\left[\beta_{g}\right]\right\rangle$ неподвижны при действии $G$. Остальные орбиты описываются следующим образом. Положим $V:=\mathbb{Z}_{2}\left\langle\left[\beta_{1}\right], \ldots,\left[\beta_{g}\right]\right\rangle$ и $U:=\mathbb{Z}_{2}\left\langle\left[\alpha_{1}\right], \ldots,\left[\alpha_{g}\right]\right\rangle$. Тогда для любого элемента $u \in \mathcal{H}_{g}$ с нетривиальной проекцией на $U$ вдоль $V$ его $G$-орбита $G \cdot u$ состоит из всех векторов $u+v$, где $v$ пробегает $V$. В частности, $\sum_{v \in V} n_{u+v}=0$ по теореме 2.14. Положим $V_{1}:=\mathbb{Z}_{2}\left\langle\left[\beta_{2}\right], \ldots,\left[\beta_{g}\right]\right\rangle$. Тогда в специальном случае $u=\left[\alpha_{1}\right]$ мы получаем $\sum_{v \in V_{1}}\left(n_{\left[\alpha_{1}\right]+v}+n_{\left[\alpha_{1}+\beta_{1}\right]+v}\right)=0$. Возможность выбора произвольного $\mathbb{Z}_{2}$-симплектического базиса дает еще два соотношения:

$$
\sum_{v \in V_{1}}\left(n_{\left[\beta_{1}\right]+v}+n_{\left[\alpha_{1}+\beta_{1}\right]+v}\right)=0, \quad \sum_{v \in V_{1}}\left(n_{\left[\alpha_{1}\right]+v}+n_{\left[\beta_{1}\right]+v}\right)=0 .
$$

Сравнивая их, мы получаем равенство

$$
\sum_{v \in V_{1}} n_{w+v}=0
$$

сначала для $w=\left[\alpha_{1}\right],\left[\beta_{1}\right],\left[\alpha_{1}+\beta_{1}\right]$, а затем для любого $w \in \mathcal{H}_{g}, \mathbb{Z}_{2}$-ортогонального классам $\beta_{2}, \ldots, \beta_{g}$. Положим далее $V_{k}:=\mathbb{Z}_{2}\left\langle\left[\beta_{k+1}\right], \ldots,\left[\beta_{g}\right]\right\rangle$ и запишем последнее равенство для $w=\left[\beta_{1}\right]$ в виде

$$
\sum_{v \in V_{2}}\left(n_{\left[\beta_{1}\right]+v}+n_{\left[\beta_{1}+\beta_{2}\right]+v}\right)=0 .
$$

Используя приведенное выше рассуждение, получаем, что $\sum_{v \in V_{2}} n_{w+v}=0$ сначала также для $w=\left[\beta_{1}\right]$, а затем для всех $w \in \mathcal{H}_{g}, \mathbb{Z}_{2}$-ортогональных $V_{2}$. Повторяя это построение, мы получаем равенства $n_{w}=0$ для всех $w \in \mathcal{H}_{g}$. Это доказывает тривиальность $z \in \mathbb{Z}\left\langle\mathcal{H}_{g}\right\rangle$.

Свойство $Q^{\prime} \cap \mathbb{Z}\left\langle\mathcal{H}_{g}\right\rangle=1$ показывает, что абелианизация $\Lambda:=Q_{\mathrm{ab}}=Q / Q^{\prime}$ включается в расширение (2.8). Поскольку цепной элемент $C$ и элемент $\Delta^{2}\left(\mathrm{E}_{6}\right)$ лежат в $\mathbb{Z}\left\langle\mathcal{H}_{g}\right\rangle$, мы можем в описании расширения (2.8) заменить $L_{0}$ на $\Delta\left(\mathrm{E}_{7}\right)$. Квадрат $\Delta^{2}\left(\mathrm{E}_{7}\right)$ равен сумме $\sum_{t \in \mathcal{T}\left(\mathrm{E}_{7}\right)} A_{t}$. Множество квазиотражений $\mathcal{T}\left(\mathrm{E}_{7}\right)$ совпадает с множеством $\mathcal{H}_{3}$ ненулевых векторов в $\mathbb{Z}_{2}\left\langle\left[\alpha_{1}\right], \ldots,\left[\beta_{3}\right]\right\rangle$. Действуя группой $\boldsymbol{S p}\left(2 g, \mathbb{Z}_{2}\right)$, мы получаем требуемое описание образующих группы $\mathrm{W} \mathcal{I}_{g, \text { ab }}=\Lambda_{g} / \mathbb{Z}\left\langle\mathcal{H}_{g}\right\rangle$.

Для доказательства изоморфизма $W \mathcal{I}_{g, \text { ab }} \cong \wedge^{6} \mathrm{H}_{1}\left(\Sigma, \mathbb{Z}_{2}\right)$ найдем $\wp^{6}\left(\Delta^{2}\left(\mathrm{E}_{7}\right)\right)$. Явное вычисление с использованием биномиальной формулы (2.7) показывает, что $\wp^{6}\left(\Delta^{2}\left(\mathrm{E}_{7}\right)\right)=\alpha_{1} \bullet \alpha_{2} \bullet \alpha_{3} \bullet \beta_{1} \bullet \beta_{2} \bullet \beta_{3}$. Заметим еще, что над кольцом $\mathbb{Z}_{2}$ выполнено соотношение $v \bullet v=0$. Отсюда следует, что алгебра, порожденная $\mathrm{H}_{1}\left(\Sigma, \mathbb{Z}_{2}\right)$ и •-умножением, есть просто $\mathbb{Z}_{2}$-грассманова алгебра пространства $\mathrm{H}_{1}\left(\Sigma, \mathbb{Z}_{2}\right)$. Следовательно, •-произведение $\alpha_{1} \bullet \alpha_{2} \bullet \alpha_{3} \bullet \beta_{1} \bullet \beta_{2} \bullet \beta_{3}$ можно отождествить с внешним произведением $\alpha_{1} \wedge \alpha_{2} \wedge \alpha_{3} \wedge \beta_{1} \wedge \beta_{2} \wedge \beta_{3} \in$ $\wedge{ }^{6} \mathrm{H}_{1}\left(\Sigma, \mathbb{Z}_{2}\right)$. Обратно, всякое симплектическое подпространство $V \subset \mathrm{H}_{1}\left(\Sigma, \mathbb{Z}_{2}\right)$ размерности 6 имеет вид $V=x \cdot \mathbb{Z}_{2}\left\langle\left[\alpha_{1}\right], \ldots,\left[\beta_{3}\right]\right\rangle$ для некоторого преобразования $x \in \boldsymbol{S p}\left(2 g, \mathbb{Z}_{2}\right)$. Для такого подпространства $V$ элемент $x * \Delta^{2}\left(\mathrm{E}_{7}\right)$ равен сумме $\sum_{v \neq 0 \in V} A_{v}=: L_{V} \in \mathbb{Z}_{2}\left\langle\mathcal{H}_{g}\right\rangle$, а $\wp^{6}\left(L_{V}\right)$ есть внешнее произведение всех векторов любого базиса подпространства $V$. Поэтому требуемый изоморфизм $W \mathcal{I}_{g, \text { ab }} \cong \wedge^{6} \mathrm{H}_{1}\left(\Sigma, \mathbb{Z}_{2}\right)$ получается из того, что элементы, 
$\boldsymbol{S p}\left(2 g, \mathbb{Z}_{2}\right)$-сопряженные $\alpha_{1} \wedge \alpha_{2} \wedge \alpha_{3} \wedge \beta_{1} \wedge \beta_{2} \wedge \beta_{3}$, порождают пространство $\wedge{ }^{6} \mathrm{H}_{1}\left(\Sigma, \mathbb{Z}_{2}\right)$.

Теперь мы опишем второй инструмент, используемый при изучении группы $\mathbb{Z}\left\langle\mathcal{H}_{g}\right\rangle$. Сопоставим гомоморфизму $\varphi_{\mu}=(\mu \cap \cdot): \mathrm{H}_{1}\left(\Sigma, \mathbb{Z}_{2}\right) \rightarrow \mathbb{Z}_{2}$ гомоморфизм $\widehat{\varphi}_{\mu}: \mathbb{Z}\left\langle\mathcal{H}_{g}\right\rangle \rightarrow \mathbb{Z}$, положив $\widehat{\varphi}_{\mu}\left(A_{v}\right):=1 \in \mathbb{Z}$, если $\varphi_{\mu}(v) \equiv 1 \in \mathbb{Z}_{2}$, и $\widehat{\varphi}_{\mu}\left(A_{v}\right):=0$, если $\varphi_{\mu}(v) \equiv 0$, где $v \in \mathcal{H}_{g} \subset \mathrm{H}_{1}\left(\Sigma, \mathbb{Z}_{2}\right)$. Таким образом, $\widehat{\varphi}_{\mu}$ есть алгебраическое число образующих $A_{v}$, для которых $\varphi_{\mu}(v) \equiv 1$. Этот гомоморфизм очевидным образом продолжается до гомоморфизма $\widehat{\varphi}_{\mu}: \mathrm{P}\left(\mathcal{S}_{g}\right) \rightarrow \mathbb{Z}$.

ЛЕмма 2.27. Пусть $\widehat{\varphi}_{\mu}: \mathbb{Z}\left\langle\mathcal{H}_{g}\right\rangle \rightarrow \mathbb{Z}-$ гомоморбизм, индуцированный гомоморбизмом $\varphi_{\mu}: \mathrm{H}_{1}\left(\Sigma, \mathbb{Z}_{2}\right) \rightarrow \mathbb{Z}_{2}$.

i) Для любого иепного элемента $C$ значение $\widehat{\varphi}_{\mu}(C)$ равно 0 или -4 .

ii) Для квадрата любого модифицированного фонарного элемента $L^{2}$ значение $\widehat{\varphi}_{\mu}\left(L^{2}\right)$ равно 0 или -8 .

iii) Для любого элемента $x$, соответствующего скручиванию Дена $T_{\delta}$ omносительно разбивающей кривой $\delta$, выполнено $\widehat{\varphi}_{\mu}(x) \equiv 0 \bmod 4$.

iv) Выполнено сравнение $\widehat{\varphi}_{\mu}\left(\left[T_{\beta_{g}}, \Delta^{2}\left(\mathrm{~A}_{2 g}\right)\right]\right) \equiv(2 g-2) \varphi_{\mu}\left(\beta_{g}\right) \bmod 4$.

СледСтвие 2.28. Гомоморфизм $\widehat{\varphi}_{\mu}: \mathbb{Z}\left\langle\mathcal{H}_{g}\right\rangle \rightarrow \mathbb{Z}$ продолжается на стабилизатор $\operatorname{Br}\left(\mathcal{S}_{g}\right)_{\mu}$ элемента $\mu$. Это продолжение индуиирует гомоморфизм $\widehat{\varphi}_{\mu}: \operatorname{Map}_{g, 1, \mu} \rightarrow \mathbb{Z}_{4}$.

ДОКАЗАТЕЛЬСТво ЛЕммы 2.27 и СЛЕДСТвия 2.28. Начнем с доказательства следствия. Напомним, что решетка $\Lambda_{g}$ (см. предложение 2.26 , ii)) порождена множеством $\mathcal{H}_{g}$ и (образами) модифицированных фонарных элементов. Из утверждения іi) леммы немедленно следует, что существует естественное продолжение $\widehat{\varphi}_{\mu}: \Lambda_{g} \rightarrow \mathbb{Z}$, при котором $\widehat{\varphi}_{\mu}(L)=0$ или $\widehat{\varphi}_{\mu}(L)=-4$ для любого модифицированного фонарного элемента $L$.

Группа $\widehat{\boldsymbol{S p}}\left(2 g, \mathbb{Z}_{2}\right)$ (см. предложение 2.26, iii)) действует естественным образом на пространстве $\mathrm{H}_{1}\left(\Sigma, \mathbb{Z}_{2}\right)$. Пусть $\widehat{\boldsymbol{S p}}\left(2 g, \mathbb{Z}_{2}\right)_{\mu}$ - стабилизатор класса $\mu$. Из предложения 2.26, iv) следует, что $\widehat{\boldsymbol{S p}}\left(2 g, \mathbb{Z}_{2}\right)_{\mu}$ есть образ группы $\operatorname{Br}\left(\mathcal{S}_{g}\right)_{\mu}$ при естественном эпиморфизме $\operatorname{Br}\left(\mathcal{S}_{g}\right) \rightarrow \widehat{\boldsymbol{S p}}\left(2 g, \mathbb{Z}_{2}\right)$. Следующий шаг в доказательстве следствия - продолжение $\widehat{\varphi}_{\mu}$ до гомоморфизма $\widehat{\varphi}_{\mu}: \widehat{\boldsymbol{S p}}\left(2 g, \mathbb{Z}_{2}\right)_{\mu} \rightarrow \mathbb{Z}$. Заметим, что группа $\boldsymbol{S p}\left(2 g, \mathbb{Z}_{2}\right)_{\mu}$ порождается (образами) скручиваний Дена $T_{\delta}$ с $\delta \cap \mu \equiv 0 \bmod 2$. В силу предложения 2.26 группа $\widehat{\boldsymbol{S p}}\left(2 g, \mathbb{Z}_{2}\right)_{\mu}$ порождена квадратами скручиваний Дена $T_{\delta}^{2}$ (относительно неразбивающих кривых $\delta$ ), образами фонарных элементов и скручиваниями Дена $T_{\delta}$, для которых $\delta \cap \mu \equiv 0 \bmod 2$. Положим $\widehat{\varphi}_{\mu}\left(T_{\delta}\right)=0$ для таких скручиваний Дена. Чтобы проверить корректность этого определения, рассмотрим элемент $x$, являющийся произведением скручиваний $T_{\delta_{j}}$ при $\delta_{j} \cap \mu \equiv 0 \bmod 2$ и лежащий в $\Lambda_{g}$. Квадрат этого элемента $x^{2}$ лежит в $\mathbb{Z}\left\langle\mathcal{H}_{g}\right\rangle$. Далее, пусть $x=T_{\delta_{1}}^{\epsilon_{1}} \cdot x_{1}$, тогда

$$
x^{2}=T_{\delta_{1}}^{\epsilon_{1}} \cdot x_{1} \cdot T_{\delta_{1}}^{\epsilon_{1}} \cdot x_{1}=\left(T_{\delta_{1}}^{\epsilon_{1}}\right)^{2} \cdot\left[T_{\delta_{1}}^{-\epsilon_{1}}, x_{1}\right] \cdot x_{1}^{2} .
$$

Повторяя эту процедуру, мы видим, что $x^{2}$ есть произведение квадратов $\left(T_{\delta_{i}}^{\epsilon_{i}}\right)^{2}$ скручиваний Дена, $\delta_{i} \cap \mu \equiv 0 \bmod 2$, и коммутаторов $\left[y_{2 j-1}, y_{2 j}\right]$, для которых проекции элементов $y_{j}$ в группу $\mathrm{W}\left(\mathcal{S}_{g}\right)$ принадлежат стабилизатору элемента $\mu$. Значит, из теоремы 2.14 следует, что $\widehat{\varphi}_{\mu}\left(x^{2}\right)=0$. Таким образом, $\widehat{\varphi}_{\mu}(x)=0$, 
следовательно, гомоморфизм $\widehat{\varphi}_{\mu}: \widehat{\boldsymbol{S p}}\left(2 g, \mathbb{Z}_{2}\right)_{\mu} \rightarrow \mathbb{Z}$ действительно определен корректно.

Смысл леммы состоит в том, что гомоморфизм $\widehat{\varphi}_{\mu}: \widehat{\boldsymbol{S p}}\left(2 g, \mathbb{Z}_{2}\right)_{\mu} \rightarrow \mathbb{Z}$ принимает значения в $4 \mathbb{Z}$ на образах всех элементов, отвечающих соотношениям в группе классов отображений Мар ${ }_{g, 1}$. Это свойство эквивалентно существованию индуцированного гомоморфизма $\widehat{\varphi}_{\mu}: \operatorname{Map}_{g, 1, \mu} \rightarrow \mathbb{Z}_{4}$.

Теперь перейдем к доказательству леммы.

i) Очевидно, что имеется следующее свойство сопряжения: $\widehat{\varphi}_{x * \mu}(x * A)=$ $\widehat{\varphi}_{\mu}(A)$ для любого $x \in \boldsymbol{S p}\left(2 g, \mathbb{Z}_{2}\right)$ и любого $A \in \mathbb{Z}\left\langle\mathcal{H}_{g}\right\rangle$. Значит, мы можем считать, что $C$ - базисный цепной элемент $C_{0}:=\Delta^{-4}\left(\mathrm{~A}_{4}\right) \Delta^{2}\left(\mathrm{~A}_{5}\right)$. Далее, ограничивая $\varphi_{\mu}$ на подпространство $\mathbb{Z}_{2}\left\langle\alpha_{1}, \alpha_{2}, \beta_{1}, \beta_{2}\right\rangle$, мы можем считать, что $g=2$ и что это ограничение нетривиально.

Нам понадобятся стандартные факты о системах корней (см. [15]). В частности, мы имеем естественное отождествление систем Кокстера $\mathcal{S}_{2}=\mathrm{A}_{5}$ и соответствующих групп $\boldsymbol{S} \boldsymbol{p}\left(4, \mathbb{Z}_{2}\right)=\mathrm{W}\left(\mathrm{A}_{5}\right)$. Кроме того, множество $\mathcal{H}_{2}$ естественно отождествляется с множеством $\Phi^{+}\left(\mathrm{A}_{5}\right)$ положительных корней системы $\mathrm{A}_{5}$.

Мы утверждаем, что действие группы $\mathrm{W}\left(\mathrm{A}_{4}\right)$ на $\Phi^{+}\left(\mathrm{A}_{5}\right)$ имеет ровно две орбиты. Первая из них - это система корней $\Phi^{+}\left(\mathrm{A}_{4}\right)$. Явное описание систем корней дает пять оставшихся элементов: $\left\{v_{1}, v_{1}+v_{2}, v_{1}+v_{2}+v_{3}, \ldots, v_{1}+\cdots\right.$ $\left.\cdots+v_{4}+v_{0}\right\}$. (Здесь мы используем обозначения из определения 2.18 и обозначаем через $v_{i}$ простой корень, соответствующий $s_{i}, i=0 ; 1, \ldots, 4$.) Непосредственно видно, что $v_{1}+v_{2}$ получается из $v_{1}$ применением отражения $s_{2}=T_{a_{2}}$, $v_{1}+v_{2}+v_{3}$ получается из $v_{1}+v_{2}$ применением $s_{3}=T_{a_{3}}$ и т. д. Отсюда вытекает требуемое описание орбит.

Следовательно, достаточно вычислить $\widehat{\varphi}_{\mu}\left(\Delta^{-4}\left(\mathrm{~A}_{4}\right) \Delta^{2}\left(\mathrm{~A}_{5}\right)\right)$ в случаях $\mu=$ $\beta_{1}=a_{1}$ и $\mu=\beta_{2}=a_{0}$. По определению значение $\widehat{\varphi}_{\mu}\left(\Delta^{2}\left(\mathrm{~A}_{5}\right)\right)$ равно числу векторов $v \in \mathrm{H}_{1}\left(\Sigma, \mathbb{Z}_{2}\right)$, для которых $\varphi_{\mu}(v)=1$. Таковых имеется $2^{3}=8$, т. е. $\widehat{\varphi}_{\mu}\left(\Delta^{2}\left(\mathrm{~A}_{5}\right)\right)=8$.

Для упрощения обозначений заменим систему $\left\{s_{2}, s_{3}, s_{4}, s_{0}\right\}$ на $\left\{s_{1}, s_{2}, s_{3}, s_{4}\right\}$. (Такая замена возможна при $g=2$ в силу изоморфизма этих систем.) Тогда $\Delta^{2}\left(\mathrm{~A}_{4}\right)=\sum_{1 \leqslant j \leqslant k \leqslant 4} A_{v_{j k}}$, где $v_{j k}:=\sum_{i=j}^{k} a_{i} \in \mathrm{H}_{1}\left(\Sigma, \mathbb{Z}_{2}\right)$. Значит, $\widehat{\varphi}_{\beta_{1}}\left(\Delta^{2}\left(\mathrm{~A}_{4}\right)\right)-$ число векторов $v_{j k}$, "содержащих" слагаемое $\alpha_{1}=a_{2}$. Для таких векторов $1 \leqslant j \leqslant 2 \leqslant k \leqslant 4$, так что $\widehat{\varphi}_{\beta_{1}}\left(\Delta^{2}\left(\mathrm{~A}_{4}\right)\right)=2 \times 3=6$ и $\widehat{\varphi}_{\beta_{1}}\left(\Delta^{4}\left(\mathrm{~A}_{4}\right)\right)=12$. Аналогичное рассуждение в случае $\mu=\beta_{2}$ приводит к векторам $v_{j k}$, где $1 \leqslant$ $j \leqslant k=4$, откуда следует, что $\widehat{\varphi}_{\beta_{2}}\left(\Delta^{4}\left(\mathrm{~A}_{4}\right)\right)=4 \times 2=8$. Итак, значение $\widehat{\varphi}_{\mu}(C)$ равно либо $8-8=0$, либо $8-12=-4$.

ii) Рассмотрим теперь квадрат модифицированного фонарного элемента $L^{2}$. Мы утверждаем, что предыдущее рассуждение остается верным, если в нем заменить $\mathrm{A}_{4}$ на $\mathrm{E}_{6}$ и $\mathrm{A}_{5}$ на $\mathrm{E}_{7}$. В частности, имеются естественные отождествления систем Кокстера $\mathcal{S}_{3}=\mathrm{E}_{7}$ и множеств положительных корней $\Phi^{+}\left(\mathrm{E}_{7}\right)=\mathcal{H}_{3}$, а также изоморфизм групп $\mathrm{W}\left(\mathrm{E}_{7}\right)=\boldsymbol{S p}\left(6, \mathbb{Z}_{2}\right) \times \mathbb{Z}_{2}\left\langle w_{\circ}\left(\mathrm{E}_{7}\right)\right\rangle$. Применяя сопряжение, мы сводим задачу к случаю базисного фонарного элемента $\Delta^{-4}\left(\mathrm{E}_{6}\right) \Delta^{2}\left(\mathrm{E}_{7}\right)$. Аналогично предыдущей ситуации мы можем считать, что $g=3$ и ограничение $\varphi_{\mu}$ на соответствующее подпространство нетривиально. Тогда снова $\widehat{\varphi}_{\mu}\left(\Delta^{2}\left(\mathrm{E}_{7}\right)\right)$ есть число векторов $v \in \mathrm{H}_{1}\left(\Sigma, \mathbb{Z}_{2}\right)$, удовлетворяющих условию $\varphi_{\mu}(v)=1$. На этот раз это число равно $2^{5}=32=\widehat{\varphi}_{\mu}\left(\Delta^{2}\left(\mathrm{E}_{7}\right)\right)$. 
Теперь опишем $\mathrm{W}\left(\mathrm{E}_{6}\right)$-орбиты в $\mathcal{T}\left(\mathrm{E}_{7}\right)$. Поскольку квазиотражения находятся во взаимно однозначном соответствии с положительными корнями алгебры Ли, имеется 63 квазиотражения в $\mathcal{T}\left(\mathrm{E}_{7}\right)$ и 36 квазиотражений в $\mathcal{T}\left(\mathrm{E}_{6}\right)$. Далее, вложение систем Кокстера $\mathrm{E}_{6} \subset \mathrm{E}_{7}$ индуцирует вложение множеств квазиотражений. Таким образом, $\mathcal{T}\left(\mathrm{E}_{6}\right)$ является $\mathrm{W}\left(\mathrm{E}_{6}\right)$-орбитой в $\mathcal{T}\left(\mathrm{E}_{7}\right)$, и имеется еще $63-36=27$ квазиотражений.

Одним из оставшихся квазиотражений является элемент $s_{1}$. (Мы используем обозначения из определения 2.18.) Для того чтобы найти его $\mathrm{W}\left(\mathrm{E}_{6}\right)$-орбиту, рассмотрим комплексную алгебру Ли $\mathfrak{g}$ типа $\mathrm{E}_{7}$. Зафиксируем картановскую подалгебру $\mathfrak{h} \subset \mathfrak{g}$ и согласованную систему простых корней, которые находятся во взаимно однозначном соответствии с системой $\mathrm{E}_{7}=\left\{s_{0} ; s_{1}, \ldots, s_{6}\right\}$. Для любого $t \in \mathcal{T}\left(\mathrm{E}_{7}\right)$ обозначим через $\alpha_{t} \in \mathfrak{h}^{*}$ соответствующий положительный корень, а через $\mathfrak{g}_{t}^{ \pm}-$корневые подпространства, соответствующие $\pm \alpha_{t}$. Далее, пусть $\mathfrak{g}^{\prime} \subset \mathfrak{g}-$ подалгебра Ли типа $\mathrm{E}_{6}$, определенная вложением $\mathrm{E}_{6} \subset \mathrm{E}_{7}$, a $\mathfrak{h}^{\prime} \subset \mathfrak{h}-$ согласованное вложение картановской подалгебры $\mathfrak{h}^{\prime}$. Обозначим через $B: \mathfrak{h}^{*} \times \mathfrak{h}^{*} \rightarrow \mathbb{C}$ каноническую билинейную форму на $\mathfrak{h}^{*}$, нормализованную условием $B\left(\alpha_{s_{i}}, \alpha_{s_{i}}\right)=+2$.

Обозначим через $V^{+}$(соответственно, $V^{-}$) сумму корневых подпространств $\mathfrak{g}_{t}^{+}$(соответственно, $\mathfrak{g}_{t}^{-}$) по всем квазиотражениям $t \in \mathcal{T}\left(\mathrm{E}_{7}\right) \backslash \mathcal{T}\left(\mathrm{E}_{6}\right)$. Поскольку каждый корень $\alpha_{t}, t \in \mathcal{T}\left(\mathrm{E}_{7}\right) \backslash \mathcal{T}\left(\mathrm{E}_{6}\right)$, имеет положительный коэффициент при $\alpha_{s_{1}}$, подпространство $V^{+}$инвариантно относительно присоединенного действия $\mathfrak{g}^{\prime}$. Это означает, что вся орбита $\mathrm{W}\left(\mathrm{E}_{6}\right) \cdot \alpha_{s_{1}}$ лежит в множестве $\Phi^{+}\left(\mathrm{E}_{7}\right)$ (в отличие от действия всей группы Вейля $\mathrm{W}\left(\mathrm{E}_{7}\right)$, которое переводит каждый корень в противоположный).

Из диаграммы Дынкина мы видим, что $B\left(\alpha_{s_{1}}, \alpha_{s_{2}}\right)=-1$ и корень $\alpha_{s_{1}}$ ортогонален остальным простым корням $\alpha_{s_{0}} ; \alpha_{s_{3}}, \ldots, \alpha_{s_{6}}$ в $\mathrm{E}_{6}$. Это означает, что для любого $v \in \mathfrak{h}^{\prime}$ выполнено равенство $\alpha_{s_{1}}(v)=-\omega_{s_{2}}(v)$, где $\omega_{s_{2}}-$ фундаментальный вес системы $\mathrm{E}_{6}$, двойственный к $\alpha_{s_{2}}$. Следовательно, подпространство $V^{+}$ содержит неприводимый $\mathfrak{g}^{\prime}$-подмодуль с минимальным весом $-\omega_{s_{2}}$. Известно, что размерность этого подмодуля равна 27. Значит, $\operatorname{dim} V^{+} \geqslant 27$. Аналогично, $\operatorname{dim} V^{-} \geqslant 27$. Сравнивая размерности, мы заключаем, что $\mathfrak{g}^{\prime}$-неприводимое разложение алгебры $\mathfrak{g}$ имеет вид $\mathfrak{g}=\mathfrak{g}^{\prime} \oplus V^{+} \oplus V^{-} \oplus \mathbb{C}\left\langle\alpha_{s_{1}}^{\vee}\right\rangle$, где $\alpha_{s_{1}}^{\vee}$ кокорень, двойственный корню $\alpha_{s_{1}}$. Заметим теперь, что согласно [15, гл. VI, упражнение $24 \mathrm{k} \S 1]$ вес $-\omega_{s_{2}}$ является микровесом ${ }^{2}$. Последнее свойство эквивалентно тому, что $\mathfrak{g}^{\prime}$-веса в $V^{+}$образуют одну $\mathrm{W}\left(\mathrm{E}_{6}\right)$-орбиту.

Вернемся к вычислению возможных значений $\widehat{\varphi}_{\mu}\left(\Delta^{2}\left(\mathrm{E}_{6}\right)\right)$. Воспользуемся следующей явной конструкцией системы корней типа $\mathrm{E}_{6}$. Пусть $\left(\mathfrak{h}^{\prime}\right)_{\mathbb{R}}^{*}$ (вещественная форма картановской подалгебры $\left.\left(\mathfrak{h}^{\prime}\right)^{*}\right)$ есть векторное пространство, натянутое на векторы $\varepsilon_{1}, \ldots, \varepsilon_{6} ; \varepsilon$, удовлетворяющие линейному соотношению $\varepsilon_{1}+\cdots+\varepsilon_{6}=0$. Определим билинейную форму на $\left(\mathfrak{h}^{\prime}\right)_{\mathbb{R}}^{*}$, положив $B\left(\varepsilon_{i}, \varepsilon_{i}\right)=\frac{5}{6}$, $B\left(\varepsilon_{i}, \varepsilon_{j}\right)=-\frac{1}{6}, B(\varepsilon, \varepsilon)=\frac{1}{2}$ и $B\left(\varepsilon_{i}, \varepsilon\right)=0$. Тогда множество

$$
\Phi:=\left\{ \pm 2 \varepsilon, \varepsilon_{i}+\varepsilon_{j}+\varepsilon_{k} \pm \varepsilon, i>j>k ; \varepsilon_{i}-\varepsilon_{j}, i \neq j\right\}
$$

является системой корней типа $\mathrm{E}_{6}$, а множество

$$
\pi:=\left\{\alpha_{s_{i}}:=\varepsilon_{i}-\varepsilon_{i-1}, i=2, \ldots, 6 ; \alpha_{s_{0}}:=\varepsilon_{1}+\varepsilon_{2}+\varepsilon_{3}+\varepsilon\right\}
$$

\footnotetext{
${ }^{2}$ Автор благодарен В. Зёргелю за эту ссылку.
} 
является системой простых корней относительно подходящей камеры Вейля. Соответствующие положительные корни образуют множество

$$
\Phi^{+}:=\left\{2 \varepsilon, \varepsilon_{i}+\varepsilon_{j}+\varepsilon_{k}+\varepsilon, i>j>k ; \varepsilon_{i}-\varepsilon_{j}, i>j\right\} .
$$

Обозначим через $\Lambda$ целочисленную решетку, порожденную множеством $\boldsymbol{\pi}$. Поскольку кривые $a_{0} ; a_{2}, \ldots, a_{6}$ задают базис целочисленных гомологий поверхности $\Sigma$, мы можем отождествить решетку $\Lambda$ с $\mathrm{H}_{1}(\Sigma, \mathbb{Z})$. Как мы уже показали, любой гомоморфизм $\varphi_{\mu}: \mathrm{H}_{1}\left(\Sigma, \mathbb{Z}_{2}\right) \rightarrow \mathbb{Z}_{2}$ есть $\mathbb{Z}_{2}$-редукция либо гомоморфизма $\lambda \in \Lambda \mapsto B\left(\alpha_{t}, \lambda\right) \in \mathbb{Z}$ для некоторого $\alpha_{t} \in \Phi$, либо гомоморфизма $\lambda \in \Lambda \mapsto B(\gamma, \lambda) \in \mathbb{Z}$ для некоторого веса $\gamma$, соответствующего $\mathfrak{g}^{\prime}$-модулю $V^{+}$. Более того, гомоморфизмы $\varphi_{\mu}$ одного типа дают одинаковое значение $\widehat{\varphi}_{\mu}\left(\Delta^{2}\left(\mathrm{E}_{6}\right)\right)$.

В качестве представителя первой $\mathrm{W}\left(\mathrm{E}_{6}\right)$-орбиты возьмем корень $2 \varepsilon$. Тогда $\widehat{\varphi}_{\mu}\left(\Delta^{2}\left(\mathrm{E}_{6}\right)\right)$ равно числу корней вида $\varepsilon_{i}+\varepsilon_{j}+\varepsilon_{k}+\varepsilon$, для которых $i>j>k$. Таким образом, в этом случае $\widehat{\varphi}_{\mu}\left(\Delta^{2}\left(\mathrm{E}_{6}\right)\right)=\left(\begin{array}{l}6 \\ 3\end{array}\right)=20$. Отсюда следует, что $\widehat{\varphi}_{\mu}\left(L^{2}\right)=32-2 \times 20=-8$.

В качестве представителя второй $\mathrm{W}\left(\mathrm{E}_{6}\right)$-орбиты рассмотрим вес $\varepsilon_{1}+\varepsilon_{2}$. Для подсчета числа корней в этом случае воспользуемся следующими наблюдениями. Во-первых, поскольку корень $\varepsilon$ ортогонален $\varepsilon_{1}$ и $\varepsilon_{2}$, мы можем заменить $\varepsilon_{i}+\varepsilon_{j}+\varepsilon_{k}+\varepsilon$ на $\varepsilon_{i}+\varepsilon_{j}+\varepsilon_{k}$. Во-вторых, поскольку $\varepsilon_{1}+\cdots+\varepsilon_{6}=0$, а нас интересует только четность числа $B\left(\varepsilon_{1}+\varepsilon_{2}, \alpha_{t}\right)$, мы можем заменить каждый корень $\varepsilon_{i}+\varepsilon_{j}+\varepsilon_{k}$ на $\varepsilon_{1}+\cdots+\varepsilon_{6}-\varepsilon_{i}+\varepsilon_{j}+\varepsilon_{k}$. Таким образом, каждый корень $\varepsilon_{i}+\varepsilon_{j}+\varepsilon_{k}$ при $i>j>k \geqslant 2$ нужно посчитать дважды. Явная комбинаторика такова:

1) $\varepsilon_{2}-\varepsilon_{1}$ ортогонален $\varepsilon_{1}+\varepsilon_{2}$;

2) $B\left(\varepsilon_{1}+\varepsilon_{2}, \varepsilon_{i}-\varepsilon_{1}\right)=-1$ при $i \geqslant 3$, и таких корней 4 ;

3) $B\left(\varepsilon_{1}+\varepsilon_{2}, \varepsilon_{i}-\varepsilon_{2}\right)=-1$ при $i \geqslant 3$, и таких корней 4 ;

4) $B\left(\varepsilon_{1}+\varepsilon_{2}, \varepsilon_{i}+\varepsilon_{j}+\varepsilon_{k}\right)=-1$ при $i>j>k \geqslant 3$, и таких корней $2 \times 4=8$;

5) $B\left(\varepsilon_{1}+\varepsilon_{2}, \varepsilon_{2}+\varepsilon_{i}+\varepsilon_{j}\right)=0$ при $i>j \geqslant 3$.

Окончательно получаем, что в этом случае $\widehat{\varphi}_{\mu}\left(\Delta^{2}\left(\mathrm{E}_{6}\right)\right)=16$ и $\widehat{\varphi}_{\mu}\left(L^{2}\right)=$ $32-2 \times 16=0$.

iii) Каждая разбивающая кривая $\gamma \subset \Sigma \backslash\left\{z_{0}\right\}$ делит поверхность $\Sigma$ на две части $\Sigma^{\prime}$ и $\Sigma^{\prime \prime}$, одна из которых (скажем, $\Sigma^{\prime \prime}$ ) содержит точку $z_{0}$. Пусть $p \geqslant 1$ - род другой части $\Sigma^{\prime}$. Тогда вся конфигурация сопряжена такой, в которой часть $\Sigma^{\prime}$ содержит кривые $a_{1}, \ldots, a_{2 p}$, а часть $\Sigma^{\prime \prime}-$ кривые $a_{2 p+2}, \ldots, a_{2 g}$ (см. расположение кривой $\gamma_{3}$ на рис. 3). В частности, если $p=g$, то кривая $\gamma$ окружает базовую точку $z_{0}$ и соответствует граничной кривой $\partial$, представленной на рис. 3. В силу (на этот раз разбивающего) цепного соотношения имеем $T_{\gamma_{p}} T_{\gamma_{p-1}}=\left(T_{\beta_{p}} T_{\alpha_{p}} T_{\beta_{p}^{\prime}}\right)^{4}$, где кривая $\beta_{p}^{\prime}$ аналогична кривой, изображенной на рис. 1 , и мы считаем, что $T_{\gamma_{0}}=1 \in$ Map $_{g, 1}$ в специальном случае $p=1$. Таким образом, скручивание $T_{\gamma}$ сопряжено произведению элементов $\left(T_{\beta_{p}} T_{\alpha_{p}} T_{\beta_{p}^{\prime}}\right)^{4}$. Отношения инцидентности в конфигурации $\beta_{p}, \alpha_{p}, \beta_{p}^{\prime}$ соответствуют системе Кокстера $\mathrm{A}_{3}$, а произведение $\left(T_{\beta_{p}} T_{\alpha_{p}} T_{\beta_{p}^{\prime}}\right)^{4}$ равно квадрату элемента Гарсайда $\Delta^{2}\left(\mathrm{~A}_{3}\right)$. Следовательно, элемент $\left(T_{\beta_{p}} T_{\alpha_{p}} T_{\beta_{p}^{\prime}}\right)^{4}$ лежит в группе $\mathrm{P}\left(\mathcal{S}_{g}\right)$ и равен в $\mathbb{Z}\left\langle\mathcal{H}_{g}\right\rangle$ сумме $\sum_{1 \leqslant i \leqslant j \leqslant 3} A_{v_{i j}}$, где $v_{11}=\left[\beta_{p}\right] \in \mathrm{H}_{1}\left(\Sigma, \mathbb{Z}_{2}\right)$, $v_{22}=\left[\alpha_{p}\right], v_{33}=\left[\beta_{p}^{\prime}\right]=\left[\beta_{p}\right]$ и $v_{i j}=\sum_{k=i}^{j} v_{k k}$. Явное вычисление дает 
$\sum_{1 \leqslant i \leqslant j \leqslant 3} A_{v_{i j}}=2 A_{\left[\beta_{p}\right]}+2 A_{\left[\alpha_{p}\right]}+2 A_{\left[\alpha_{p}+\beta_{p}\right]}$. Значит, гомоморфизм $\widehat{\varphi}_{\mu}$ принимает значение 0 или 4 на любом произведении вида $\left(T_{\beta_{p}} T_{\alpha_{p}} T_{\beta_{p}^{\prime}}\right)^{4}$. Это доказывает утверждение iii).

iv) Подгруппа $\partial_{\pi}\left(\pi_{1}\left(\Sigma, z_{0}\right)\right) \subset$ Мар $_{g, 1}$ нормально порождена базисным гиперэллиптическим соотношением $\left[T_{\beta_{g}}, \Delta^{2}\left(\mathrm{~A}_{2 g}\right)\right]$. Его геометрическая реализация это $\partial_{\pi}\left(\beta_{g}^{\prime \prime}\right)$, где элемент $\beta_{g}^{\prime \prime} \in \pi_{1}\left(\Sigma, z_{0}\right)$ представляется вложенной кривой, также обозначаемой $\beta_{g}^{\prime \prime}$, которая изотопна кривой $\beta_{g}$ и проходит через базовую точку $z_{0}$. На рис. 3 эта кривая соответствует дуге $\beta_{g}^{\prime \prime}$, а правильная картинка для замкнутой поверхности получится, если стянуть граничную окружность $\partial$ в базовую точку $z_{0}$. В частности, после такого стягивания кривые $\beta_{g}$ и $\beta_{g}^{\prime}$ ограничивают трубчатую окрестность кривой $\beta_{g}^{\prime \prime}$. Заметим, что элемент $\Delta^{2}\left(\mathrm{~A}_{2 g}\right)$ представляется гиперэллиптической инволюцией на поверхности $\Sigma$, переводящей кривую $\beta_{g}$ в кривую $\beta_{g}^{\prime}$.

Ввиду этого алгебраического описания нам достаточно найти возможные значения $\widehat{\varphi}_{\mu}\left(\left[T_{\beta_{g}}, \Delta^{2}\left(\mathrm{~A}_{2 g}\right)\right]\right)$. Заметим сначала, что в группе $\mathbb{Z}\left\langle\mathcal{H}_{g}\right\rangle$ выполнено равенство $\Delta^{2}\left(\mathrm{~A}_{2 g}\right)=\sum_{1 \leqslant i \leqslant j \leqslant 2 g} A_{v_{i j}}$, где $v_{i j}:=\sum_{k=i}^{j}\left[a_{k}\right] \in \mathrm{H}_{1}\left(\Sigma, \mathbb{Z}_{2}\right)$. Далее, $T_{\beta_{g}}\left(v_{i j}\right)=v_{i j}$ при $i \leqslant j<2 g$, так что в группе $\mathbb{Z}\left\langle\mathcal{H}_{g}\right\rangle$ мы получаем равенство

$$
\left[T_{\beta_{g}}, \Delta^{2}\left(\mathrm{~A}_{2 g}\right)\right]=\sum_{i=1}^{2 g}\left(A_{v_{i, 2 g}+\left[\beta_{g}\right]}-A_{v_{i, 2 g}}\right) .
$$

Легко видеть, что если $\varphi_{\mu}\left[\beta_{g}\right]=0$, то $\widehat{\varphi}_{\mu}\left(A_{v_{i, 2 g}+\left[\beta_{g}\right]}-A_{v_{i, 2 g}}\right)=0$ для любого $i=1, \ldots, 2 g$. Поэтому остается рассмотреть случай $\varphi_{\mu}\left[\beta_{g}\right]=1$.

Здесь мы заметим, что классы $\left[a_{1}\right], \ldots,\left[a_{2 g}\right]$ образуют базис в $\mathrm{H}_{1}\left(\Sigma, \mathbb{Z}_{2}\right)$, причем $\left[\beta_{g}\right]=\left[a_{1}\right]+\left[a_{3}\right]+\cdots+\left[a_{2 g-1}\right]$. Таким образом, гомоморфизм $\varphi_{\mu}: \mathrm{H}_{1}\left(\Sigma, \mathbb{Z}_{2}\right) \rightarrow$ $\mathbb{Z}_{2}$ полностью определяется своими значениями $\varphi_{\mu}\left[a_{1}\right], \ldots, \varphi_{\mu}\left[a_{2 g}\right]$. Значит, варьируя эти значения, мы получим всевозможные $\varphi_{\mu}$. Ясно, что группа таких преобразований гомоморфизмов $\varphi_{\mu}$, при которых не изменяется значение $\varphi_{\mu}\left[\beta_{g}\right]=1 \in \mathbb{Z}_{2}$, порождена следующими двумя типами простых преобразований: либо мы изменяем одно значение $\varphi_{\mu}\left[a_{2 k}\right]$ на кривой $a_{2 k}$ с четным номером, либо одновременно изменяем два значения $\varphi_{\mu}\left[a_{2 k-1}\right]$ и $\varphi_{\mu}\left[a_{2 k+1}\right]$ на двух следующих друг за другом кривых $a_{2 k-1}$ и $a_{2 k+1}$ с нечетными номерами. Поскольку $\varphi_{\mu}\left[\beta_{g}\right]=1$, каждое значение $\widehat{\varphi}_{\mu}\left(A_{v_{i, 2}+\left[\beta_{g}\right]}-A_{v_{i, 2} g}\right)$ есть либо +1 , либо -1 . Ключевое наблюдение состоит в том, что для любого простого преобразования мы получаем четное число изменений знака у значений $\widehat{\varphi}_{\mu}\left(A_{v_{i, 2 g}+\left[\beta_{g}\right]}-A_{v_{i, 2 g}}\right)$. А именно, $2 k$ изменений знаков происходят при изменении значения $\varphi_{\mu}\left[a_{2 k}\right]$, и два знака меняются при изменении значений $\varphi_{\mu}\left[a_{2 k-1}\right]$ и $\varphi_{\mu}\left[a_{2 k+1}\right]$. Отсюда следует, что значение $\widehat{\varphi}_{\mu}\left(\left[T_{\beta_{g}}, \Delta^{2}\left(\mathrm{~A}_{2 g}\right)\right]\right) \bmod 4$ остается неизменным, т. е. оно зависит только от $\varphi_{\mu}\left(\left[\beta_{g}\right]\right)$. Явное вычисление в случае, когда $\varphi_{\mu}$ обращается в нуль на $\left[\alpha_{1}\right], \ldots,\left[\alpha_{g}\right] ;\left[\beta_{1}\right], \ldots,\left[\beta_{g-1}\right]$, дает равенство $\widehat{\varphi}_{\mu}\left(\left[T_{\beta_{g}}, \Delta^{2}\left(\mathrm{~A}_{2 g}\right)\right]\right)=2 g-2$, что и утверждалось.

\section{3. Разложения на множители в группах классов отображений.}

В этом пункте мы рассматриваем задачи разложения на множители в группе $\operatorname{Map}_{g, 1}$ и ее подгруппе Map $_{g, 1, \mu}$, оставляющей неподвижным заданный ненулевой класс гомологий $\mu \in \mathrm{H}_{1}\left(\Sigma, \mathbb{Z}_{2}\right)$. Топологический смысл этих задач состоит 
в существовании или нетривиальности специальных классов гомологий в семействах Лефшеца.

Напомним (см. формулу $(1.15))$, что через $\widetilde{\operatorname{Map}}_{g}$ мы обозначаем группу в расширении

$$
1 \rightarrow \mathrm{H}_{1}\left(\Sigma, \mathbb{Z}_{2}\right) \rightarrow \widetilde{\operatorname{Map}}_{g} \rightarrow \operatorname{Map}_{g} \rightarrow 1
$$

т. е. $\widetilde{\operatorname{Map}}_{g}$ есть фактор группы Мap $_{g, 1}$ по образу при отображении $\partial_{\pi}$ ядра гомоморфизма $\pi_{1}\left(\Sigma, z_{0}\right) \rightarrow \mathrm{H}_{1}\left(\Sigma, \mathbb{Z}_{2}\right)$. Для нас будут важны только проекции элементов $F \in \operatorname{Map}_{g, 1}$ в группу $\widehat{\operatorname{Map}}_{g}$. Пусть $\widehat{\operatorname{Map}}_{g, \mu} \subset \widehat{\operatorname{Map}}_{g}$ - стабилизатор класса $\mu$.

ПреДЛОЖЕНИЕ 2.29. Существует гомоморфизм $\widetilde{\varphi}_{\mu}: \widetilde{\operatorname{Map}}_{g, \mu} \rightarrow \mathbb{Z}_{4}$ со следующими свойствами:

$(\varphi 1) \widetilde{\varphi}_{\mu}\left(T_{\delta}\right) \equiv 0 \bmod 4$ для скручивания Дена $T_{\delta} \operatorname{ma\kappa oго,~что~} \delta \cap \mu \equiv 0 \bmod 2$;

$(\varphi 2) \widetilde{\varphi}_{\mu}\left(T_{\delta}^{2}\right) \equiv[\delta] \cap[\mu] \bmod 4$ для любого скручивания Дена;

$(\varphi 3) \widetilde{\varphi}_{\mu}\left(\partial_{\pi}(\gamma)\right) \equiv(2 g-2) \varphi_{\mu}(\gamma) \bmod 4$ для любого элемента $\gamma \in \pi_{1}(\Sigma)$, где $\partial_{\pi}: \pi_{1}(\Sigma) \rightarrow$ Map $_{g, 1}-$ стандартное вложение;

( $\varphi 4) \widetilde{\varphi}_{\mu}$ равен нулю на подгруппе $\widetilde{\operatorname{Map}}_{g, \mu}^{\prime} \subset \widetilde{\operatorname{Map}}_{g, \mu}$, порожденной скручиваниями Дена $T_{\delta}$ такими, что $\delta \cap \mu \equiv 0 \bmod 2, u$ коммутаторами $\left[F, F^{\prime}\right]$, где $F, F^{\prime} \in \widehat{\operatorname{Map}}_{g, \mu}$;

$(\varphi 5)$ ограничение $\widetilde{\varphi}_{\mu}$ на ядро $\operatorname{Ker}\left(\widetilde{\operatorname{Map}}_{g, \mu} \rightarrow \boldsymbol{S p}\left(2 g, \mathbb{Z}_{2}\right)\right)$ есть редукция $\bmod 4$ гомоморфизма $\widehat{\varphi}_{\mu}$.

Свойство $(\varphi 2)$ понимается следующим образом: $\widetilde{\varphi}_{\mu}\left(T_{\delta}^{2}\right) \equiv 0 \bmod 4$, если $[\delta] \cap$ $[\mu] \equiv 0 \bmod 2$, и $\widetilde{\varphi}_{\mu}\left(T_{\delta}^{2}\right) \equiv+1 \bmod 4$, если $[\delta] \cap[\mu] \equiv 1 \bmod 2$.

ДоКАЗАТЕЛЬСТвО ПРЕДЛОЖЕНИЯ 2.29. Нужно применить лемму 2.27 и следствие 2.28 .

TEOPEма 2.30. Пусть род $g=2 g^{\prime} \geqslant 2$ четен, и пусть id $=\prod_{i}\left[F_{2 i-1}, F_{2 i}\right]$ 。 $\prod_{j} T_{\delta_{j}}$ - разложение на множители в группе $\mathrm{Map}_{g}$, где $\left[F_{2 i-1}, F_{2 i}\right]$ - коммутатор элементов $F_{2 i-1}, F_{2 i} \in \operatorname{Map}_{g} u T_{\delta_{j}}-$ скручивания Дена относительно окружностей $\delta_{1}, \ldots, \delta_{n} \subset \Sigma \backslash\left\{z_{0}\right\}$. Тогда существуют подбемь $\widetilde{F}_{2 i-1}, \widetilde{F}_{2 i} \in \operatorname{Map}_{g, 1}$ и окружности $\delta_{j}^{\prime}$ в $\Sigma \backslash\left\{z_{0}\right\}$ такие, что $\delta_{j}^{\prime}$ изотопна $\delta_{i}$ на $\Sigma$, $u \prod_{i}\left[\widetilde{F}_{2 i-1}, \widetilde{F}_{2 i}\right] \cdot \prod_{j} T_{\delta_{j}^{\prime}}=\partial_{\pi} \gamma \in \operatorname{Map}_{g, 1}$ для некоторого элемента $\gamma \in \pi_{1}\left(\Sigma, z_{0}\right)$ с нулевым классом $\mathbb{Z}_{2}$-гомологий $[\gamma]=0 \in \mathrm{H}_{1}\left(\Sigma, \mathbb{Z}_{2}\right)$.

ДокАЗАтЕльство. Выберем какие-нибудь подъемы $\widetilde{F}_{i} \in \mathrm{Map}_{g, 1}$ элементов $F_{i} \in \operatorname{Map}_{g}$ и поднимем скручивания Дена $T_{\delta_{j}}$ в Map $_{g, 1}$ естественным способом.

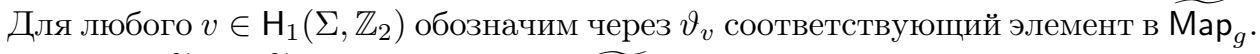
Тогда $\prod_{i}\left[\widetilde{F}_{2 i-1}, \widetilde{F}_{2 i}\right] \cdot \prod_{j} T_{\delta_{j}}=\vartheta_{w_{0}} \in \widehat{\operatorname{Map}}_{g}$ для какого-то $w_{0} \in \mathrm{H}_{1}\left(\Sigma, \mathbb{Z}_{2}\right)$.

Обозначим через $W$ множество всех $w \in \mathrm{H}_{1}\left(\Sigma, \mathbb{Z}_{2}\right)$, для которых элемент $\vartheta_{w}$ может быть представлен в виде произведения $\prod_{i}\left[\widetilde{F}_{2 i-1}, \widetilde{F}_{2 i}\right] \cdot \prod_{j} T_{\delta_{j}^{\prime}}$ для каких-то подъемов $\widetilde{F}_{i}$ и $T_{\delta_{j}^{\prime}}$. Тогда $W=w_{0}+V$ для подходящего $\mathbb{Z}_{2}$-подпространства $V \subset \mathrm{H}_{1}\left(\Sigma, \mathbb{Z}_{2}\right)$ и утверждение теоремы эквивалентно тому, что $w_{0} \in V$. Предположим противное; тогда $V$ должно быть собственным подпространством в $\mathrm{H}_{1}\left(\Sigma, \mathbb{Z}_{2}\right)$.

Пусть $\mu \in \mathrm{H}_{1}\left(\Sigma, \mathbb{Z}_{2}\right)$ - такой класс, что линейный функционал $\varphi_{\mu}: v \in$ $\mathrm{H}_{1}\left(\Sigma, \mathbb{Z}_{2}\right) \mapsto \mu \cap v \in \mathbb{Z}_{2}$ обращается в нуль на $V$, но не равен нулю на $w_{0}$. 
Мы утверждаем, что класс $\mu \in \mathrm{H}_{1}\left(\Sigma, \mathbb{Z}_{2}\right)$ инвариантен при действии всех сомножителей $F_{i}, T_{\delta_{j}}$. Из леммы 1.12 следует, что подпространство $V$ состоит из элементов, двойственных по Пуанкаре кограницам $d^{(2)}\left(\boldsymbol{\lambda}^{\vee}\right)$, где $\boldsymbol{\lambda}^{\vee}$ имеет вид (1.16). Эквивалентное двойственное условие состоит в том, что если мы рассмотрим разложение $\prod_{i}\left[F_{2 i-1}, F_{2 i}\right] \cdot \prod_{j} T_{\delta_{j}}$ как соотношение $R$, то граница $\partial_{2}\left(R \otimes_{2} \mu\right)$ обращает в нуль все такие $\lambda^{\vee}$. Используя вычисление из доказательства предложения 1.9 , получаем

$$
\begin{aligned}
\partial_{2}\left(R \otimes_{2} \mu\right)=\sum_{j} T_{\delta_{j}} & \otimes_{1} w_{j}^{T} \mu \\
& +\sum_{i}\left(F_{2 i-1} \otimes_{1}\left(F_{2 i}-\mathrm{id}\right) w_{i}^{F} \mu+F_{2 i} \otimes_{1}\left(\mathrm{id}-F_{2 i-1}\right) w_{i}^{F} \mu\right),
\end{aligned}
$$

где $w_{j}^{T}, w_{i}^{F}$ - некоторые концевые части слова $R$. Точнее, $w_{j}^{T}$ состоит из всех букв, стоящих в $R$ после $T_{\delta_{j}}$, а $w_{i}^{F}$ - из всех букв после $F_{2 i}$. Рассмотрим теперь условия $w_{j}^{T} \mu \cap \delta_{j} \equiv 0 \bmod 2$, начиная с последней буквы $T_{\delta_{l}}$ и двигаясь к началу слова $R$. По индукции мы получим требуемые равенства $\mu \cap \delta_{j} \equiv$ $0 \bmod 2$. Аналогичное рассуждение в случае условий $\left(F_{2 i}-\mathrm{id}\right) w_{i}^{F} \mu \cap \lambda_{i} \equiv 0$ и $\left(F_{2 i-1}-\mathrm{id}\right) w_{i}^{F} \mu \cap \nu_{i} \equiv 0$ дает нужный результат для $F_{i}$.

Итак, $\widetilde{\varphi}_{\mu}\left(\vartheta_{w_{0}}\right) \equiv 0 \bmod 4$ по предложению 2.29, $(\varphi 1)$. С другой стороны, $\widetilde{\varphi}_{\mu}\left(\vartheta_{w_{0}}\right) \equiv(2 g-2) \varphi_{\mu}\left(w_{0}\right) \equiv 2\left(\mu \cap w_{0}\right) \equiv 2$ по предложению $2.29,(\varphi 3)$. Полученное противоречие означает, что нужные подъемы существуют.

Из теоремы 2.30 и леммы 1.12 следует теорема 0.10, а из нее - сформулированное непосредственно после предложения 1.7 утверждение о нетривиальности класса $\mathbb{Z}_{2}$-гомологий слоя для любого топологического семейства Лефшеца со слоем четного рода.

Из доказательства теоремы 2.30 вытекает следующее утверждение.

СледСтвиЕ 2.31. Пусть род $g \geqslant 3$ нечетен, и пусть id $=\prod_{i}\left[F_{2 i-1}, F_{2 i}\right]$ 。 $\prod_{j} T_{\delta_{j}}$ - такое же разложение в группе $\mathrm{Map}_{g}$, как и в теореме 2.30. Предположим, что для любого подгема $\prod_{i}\left[\widetilde{F}_{2 i-1}, \widetilde{F}_{2 i}\right] \cdot \prod_{j} T_{\delta_{j}^{\prime}}=\partial_{\pi} \gamma \in \operatorname{Map}_{g, 1}$, где $\gamma \in \pi_{1}\left(\Sigma, z_{0}\right)$, класс $\mathbb{Z}_{2}$-гомологий $[\gamma]_{\mathbb{Z}_{2}}$ отличен от нуля. Тогда найдется класс $\mu \in \mathrm{H}_{1}\left(\Sigma, \mathbb{Z}_{2}\right)$, неподвижный при действии элементов $F_{i}$ и $T_{\delta_{j}}$ и такой, что $\mu \cap[\gamma] \equiv 1 \bmod 2$ для этого элемента $\gamma \in \pi_{1}\left(\Sigma, z_{0}\right)$.

Теорема 2.32. і) Пусть род $g \geqslant 1$ нечетен. Тогда элемент $\left(T_{\alpha_{1}} T_{\beta_{1}}\right)^{3}$ не представляется в группе $\mathrm{Map}_{g}$ в виде произведения $\prod_{i} T_{\delta_{i}}^{\epsilon_{i}} \cdot \prod_{j}\left[F_{2 j-1}, F_{2 j}\right]$ скручиваний Дена и коммутаторов, для которого $\delta_{i} \cap \beta_{1} \equiv 0 \bmod 2$ и каждий элемент $F_{j}$ сохраняет класс гомологий $\left[\beta_{1}\right]_{\mathbb{Z}_{2}} \in \mathrm{H}_{1}\left(\Sigma, \mathbb{Z}_{2}\right)$.

ii) Пусть род $g \geqslant 2$ четен. Пусть $\prod_{i} T_{\delta_{i}}^{\epsilon_{i}} \cdot \prod_{j}\left[F_{2 j-1}, F_{2 j}\right]$ - разложение элемента $\left(T_{\alpha_{1}} T_{\beta_{1}}\right)^{3}$ в группе $\mathrm{Map}_{g}$, удовлетворяюшее вышеуказанным условиям, u пусть $\widehat{F}_{j}$ - какие-то подгемы сомножителей в группу Мар ${ }_{g, 1}$. Тогда

$$
\left(T_{\alpha_{1}} T_{\beta_{1}}\right)^{-3} \prod_{i} T_{\delta_{i}}^{\epsilon_{i}} \cdot \prod_{j}\left[\widehat{F}_{2 j-1}, \widehat{F}_{2 j}\right]=\partial_{\pi}(\gamma),
$$

где $\gamma \in \pi_{1}\left(\Sigma, z_{0}\right)$ удовлетворяет условию $[\gamma] \cap \beta_{1} \equiv 1 \bmod 2$. 
ДоказАтельство. і) Рассмотрим сначала частный случай $g=1$, обозначив $\alpha_{1}, \beta_{1}$ через $\alpha, \beta$. Здесь $\mathcal{S}_{1}=\left\{s_{1}, s_{2}\right\}=\mathrm{A}_{2}$, и потому $\mathrm{W}\left(\mathcal{S}_{1}\right)=\operatorname{Sym}_{3}$ и $\operatorname{Br}\left(\mathcal{S}_{1}\right)=\mathrm{Br}_{3}$. Кроме того, мы можем отождествить Мар ${ }_{1,[1]}$ с группой кос $\mathrm{Br}_{3}$, сопоставив скручиваниям Дена $T_{\alpha}, T_{\beta}$ стандартные образующие в $\mathrm{Br}_{3}$. Помимо этого, $\mathcal{T}\left(\mathcal{S}_{1}\right)=\mathcal{H}_{1}=\{[\alpha],[\beta],[\alpha+\beta]\}$, где рассмативаются гомологии с коэффициентами в $\mathbb{Z}_{2}$. Применим теорему 2.14 к подгруппе $G:=\mathbb{Z}_{2}\left\langle T_{\beta}\right\rangle \subset$ Sym $_{3}$. Эта подгруппа имеет две орбиты в $\mathcal{T}\left(\mathcal{S}_{1}\right)$, а именно $\{[\beta]\}$ и $\{[\alpha],[\alpha+\beta]\}$. Пусть $A_{G \cdot[\beta]}, A_{G \cdot[\alpha]}$ - соответствующий базис в $\mathrm{P}_{\mathrm{ab}}\left(\mathcal{S}_{1}\right)_{G}$. Как и выше, проекция из $\mathrm{P}_{\mathrm{ab}}\left(\mathcal{S}_{1}\right)=\mathbb{Z}\left\langle A_{[\alpha]}, A_{[\beta]}, A_{[\alpha+\beta]}\right\rangle$ на компоненту $\mathbb{Z}\left\langle A_{G \cdot[\alpha]}\right\rangle$ дается гомоморфизмом $\widehat{\varphi}_{[\beta]}: \mathrm{P}_{\mathrm{ab}}\left(\mathcal{S}_{1}\right) \rightarrow \mathbb{Z}$. В этой ситуации теорема 2.14 утверждает, что $\widehat{\varphi}_{[\beta]}(F)=0$ для любого разложения $F$, удовлетворяющего условиям нашей теоремы. С другой стороны, $\left(T_{\alpha} T_{\beta}\right)^{3}$ есть элемент Гарсайда группы кос $\operatorname{Br}\left(\mathcal{S}_{1}\right)$, следовательно, его проекция в $\mathrm{P}_{\mathrm{ab}}\left(\mathcal{S}_{1}\right)$ равна $A_{[\alpha]}+A_{[\beta]}+A_{[\alpha+\beta]}$. Значит, $\widehat{\varphi}_{[\beta]}\left(\left(T_{\alpha} T_{\beta}\right)^{3}\right)=\widehat{\varphi}_{[\beta]}\left(A_{[\alpha]}+A_{[\beta]}+A_{[\alpha+\beta]}\right)=2$. Полученное противоречие доказывает невозможность такого разложения.

Теперь рассмотрим случай нечетного рода $g \geqslant 3$ и предположим, что существует удовлетворяющее условиям теоремы разложение $\prod_{i} T_{\delta_{i}}^{\epsilon_{i}} \cdot \prod_{j}\left[F_{2 j-1}, F_{2 j}\right]$. Поднимая его в группу $\operatorname{Br}\left(\mathcal{S}_{g}\right)$, мы получаем элемент $\widehat{F}$, лежащий в ядре $\operatorname{Ker}\left(\operatorname{Br}\left(\mathcal{S}_{g}\right) \rightarrow \boldsymbol{S p}\left(2 g, \mathbb{Z}_{2}\right)\right)$. Повторяя рассуждения из доказательства теоремы 2.30, мы получаем сравнение $\widehat{\varphi}_{\left[\beta_{1}\right]}(\widehat{F}) \equiv 0 \bmod 4$. Заметим, что подъем $\widehat{F}$ отличается от $\left(T_{\alpha_{1}} T_{\beta_{1}}\right)^{3}$ (рассматриваемого теперь как элемент группы $\left.\operatorname{Br}\left(\mathcal{S}_{g}\right)\right)$ на произведение цепных, фонарных и гиперэллиптических соотношений. Поскольку род $g$ нечетен, то $\widehat{\varphi}_{\left[\beta_{1}\right]}(x) \equiv 0 \bmod 4$ для любого из элементов $x$, задающих эти соотношения (включая гиперэллиптическое), а значит, значение $\widehat{\varphi}_{\left[\beta_{1}\right]}\left(\left(T_{\alpha_{1}} T_{\beta_{1}}\right)^{3}\right)$ должно делиться на 4 . С другой стороны, проделанное в случае $g=1$ вычисление показывает, что $\widehat{\varphi}_{\left[\beta_{1}\right]}\left(\left(T_{\alpha_{1}} T_{\beta_{1}}\right)^{3}\right)=2$. Противоречие.

ii) Как и раньше, применим рассуждения, использованные в доказательстве теоремы 2.30. Получится сравнение $\widehat{\varphi}_{\left[\beta_{1}\right]}\left(\left(T_{\alpha_{1}} T_{\beta_{1}}\right)^{3} \cdot \partial_{\pi}(\gamma)\right) \equiv 0 \bmod 4$. Поскольку $\widehat{\varphi}_{\left[\beta_{1}\right]}\left(\left(T_{\alpha_{1}} T_{\beta_{1}}\right)^{3}\right)=2$, нужное утверждение следует из предложения $2.29,(\varphi 3)$.

\section{§ 3. Доказательство основного результата}

В этом параграфе дается доказательство основной теоремы из введения. Сохраним обозначения из п. 1.4. В частности, $\boldsymbol{m}$ обозначает меридиан бутылки Клейна $K$, рассматриваемый как кривая на слое $\Sigma$, а $\mu=[\boldsymbol{m}]_{\mathbb{Z}_{2}}$ - гомологический класс этой кривой на $\Sigma$.

В случае, когда меридиан $\boldsymbol{m}$ разбивает поверхность $\Sigma$, утверждение доказано непосредственно после предложения 1.7. Поэтому будем предполагать, что $\boldsymbol{m}$ не разбивает $\Sigma$. Кроме того, мы можем считать, что выполнены условия предложения 1.9 и проекция класса $[K]$ на $\mathrm{H}_{1}\left(Y^{\circ}, \mathscr{H}_{1}\left(X_{y}, \mathbb{Z}_{2}\right)\right)$ тривиальна, так как в противном случае доказательство очевидно.

Рассмотрим монодромию $F_{\Gamma}$ вдоль кривой $\Gamma$. Реализуем меридиан $\boldsymbol{m}$ как кривую $\beta_{1}$ в некотором геометрическом базисе $\alpha_{1}, \beta_{1}, \ldots, \alpha_{g}, \beta_{g}$ поверхности $\Sigma$. Тогда мы можем изотопировать $F_{\Gamma}$ так, что кривая $\beta_{1}=\boldsymbol{m}$ переходит в себя при действии $F_{\Gamma}$. Индуцированное отображение $F_{\Gamma}: \beta_{1} \rightarrow \beta_{1}$ обращает ориентацию. Заметим, что отображение $\left(T_{\alpha_{1}} T_{\beta_{1}}\right)^{3}$ обладает тем же свойством на 
классах гомотопии, т. е. оно переводит свободный гомотопический класс кривой $\beta_{1}$ в себя с обратной ориентацией. Следовательно, в изотопическом классе $F_{\Gamma}^{\prime}:=F_{\Gamma}\left(T_{\alpha_{1}} T_{\beta_{1}}\right)^{-3}$ найдется представитель (который мы по-прежнему будем обозначать через $\left.F_{\Gamma}^{\prime}\right)$, сохраняющий кривую $\beta_{1}$ поточечно. В частности, $F_{\Gamma}^{\prime}$ является диффеоморфизмом поверхности $\Sigma \backslash \beta_{1}$. Из классификации диффеоморфизмов поверхностей следует, что $F_{\Gamma}^{\prime}$ можно разложить в произведение скручиваний Дена $T_{\delta}$ относительно кривых, не пересекающихся с кривой $\beta_{1}$. Зафиксируем такое разложение $F_{\Gamma}^{\prime}$ и поднимем его в группу Мар $_{g, 1}$. Обозначим полученные элементы группы $\operatorname{Map}_{g, 1}$ через $\widehat{F}_{\Gamma}^{\prime}$ и $\widehat{F}_{\Gamma}:=\widehat{F}_{\Gamma}^{\prime} \cdot\left(T_{\alpha_{1}} T_{\beta_{1}}\right)^{3}$.

3.1. Тонкая структура монодромии. В этом пункте мы уточним результат, полученный в предложении 1.9 .

ПРЕДЛОЖЕНИЕ 3.1. В условиях предложения 1.9, і) предположим дополнительно, что класс $[K] \in \mathrm{H}_{2}\left(X, \mathbb{Z}_{2}\right)$ тривиален. Тогда построенное в этом предложении разложение $\mu=\mu_{+}+\mu_{-} \in \mathrm{H}_{1}\left(\Sigma, \mathbb{Z}_{2}\right)$ удовлетворяет соотношениям

$$
\mu \cap \mu_{+} \equiv \mu \cap \mu_{-} \equiv 1 \bmod 2
$$

Кроме того, класс $\mu$ не инвариантен ни при действии группь $\pi_{1}\left(Y_{+}^{\circ}\right)$, ни при действии группы $\pi_{1}\left(Y_{-}^{\circ}\right)$ преобразованиями монодромии.

ДокАЗАТЕЛЬСтво. Необходимо исключить следующие две возможности:

1) один из классов $\mu_{ \pm}$(например, $\mu_{-}$) тривиален, так что $\mu_{+}=\mu$;

2) оба класса $\mu_{+}, \mu_{-}$нетривиальны, но $\mu \cap \mu_{+} \equiv \mu \cap \mu_{-} \equiv 0 \bmod 2$.

Сначала рассмотрим случай $\mu_{-}=0$. Тогда монодромия $F_{\Gamma}$ может быть разложена в произведение $F_{+}:=\prod_{i}\left[F_{2 i-1}, F_{2 i}\right] \cdot \prod_{j} T_{\delta_{j}}$, причем класс $\mu$ инвариантен относительно всех $F_{i}$ и всех $T_{\delta_{j}}$. Отсюда следует тождество $\left(T_{\alpha_{1}} T_{\beta_{1}}\right)^{3}=$ $F_{+} \cdot\left(F_{\Gamma}^{\prime}\right)^{-1}$, правая часть которого обладает тем же свойством. По теореме 2.32 такая ситуация невозможна в случае нечетного рода $g$, а в случае четного рода она влечет следующее утверждение. Для любого подъема $\widehat{F}_{+}$класса $F_{+}$в группу $\operatorname{Map}_{g, 1}$ имеется соотношение $\left(T_{\alpha_{1}} T_{\beta_{1}}\right)^{3}=\partial_{\pi}(\gamma) \cdot \widehat{F}_{+} \cdot\left(\widehat{F}_{\Gamma}^{\prime}\right)^{-1}$ для некоторого элемента $\gamma \in \pi_{1}(\Sigma)$, соответствующий которому класс гомологий $[\gamma] \in \mathrm{H}_{1}\left(\Sigma, \mathbb{Z}_{2}\right)$ удовлетворяет условию $[\gamma] \cap \mu \equiv 1 \bmod 2$. Выберем поднятие $\widehat{F}_{+}$как часть поднятия полной монодромии, используя теорему 2.30. Поднятие $\widehat{F}_{\Gamma} \in \operatorname{Map}_{g, 1}$ задает сечение $\sigma_{K}: \Gamma \rightarrow X$ семейства $\mathrm{pr}: X \rightarrow Y$ над кривой $\Gamma$, не пересекающееся с бутылкой Клейна $K$. С другой стороны, поднятие $\widehat{F}_{+}$определяет $\mathbb{Z}_{2}$-сечение $\left.\sigma\right|_{\Gamma}$ семейства $\mathrm{pr}: X \rightarrow Y$ над кривой $\Gamma$, которое является ограничением глобального $\mathbb{Z}_{2}$-сечения $\sigma$. Условие $[\gamma] \cap \mu \equiv 1 \bmod 2$ означает, что $\mathbb{Z}_{2}$-индекс пересечения $\sigma \cap[K]$ отличен от нуля, т. е. класс $[K]$ должен быть ненулевым. Тем самым, случай $\mu_{-}=0$ невозможен.

Предположим теперь, что оба класса $\mu_{+}$и $\mu_{-}$нетривиальны, но $\mu_{+} \cap \mu \equiv$ $\mu_{-} \cap \mu \equiv 0$. Тогда $g \geqslant 2$. Наложим дополнительное условие на выбранный выше геометрический базис $\alpha_{1}, \beta_{1}, \ldots, \alpha_{g}, \beta_{g}$ поверхности $\Sigma$. А именно, будем считать, что $\mu_{+}=\left[\beta_{2}\right]$. Поскольку группа Map $_{g}$ транзитивно действует на тройках $\left(\mu, \mu_{+}, \mu_{-}\right)$попарно ортогональных ненулевых векторов в $\mathrm{H}_{1}\left(\Sigma, \mathbb{Z}_{2}\right)$ 
с условием $\mu=\mu_{+}+\mu_{-}$, такой базис $\alpha_{1}, \beta_{1}, \ldots, \alpha_{g}, \beta_{g}$ существует. Итак, $\mu_{+} \cap$ $\left[\alpha_{1}\right] \equiv \mu_{+} \cap\left(\left[\alpha_{1}\right]+\left[\beta_{1}\right]\right) \equiv 0 \bmod 2$ и $\mu_{-} \cap\left[\alpha_{1}\right] \equiv \mu_{-} \cap\left(\left[\alpha_{1}\right]+\left[\beta_{1}\right]\right) \equiv 1 \bmod 2$. Значит, как и в доказательстве утверждения i) теоремы 2.32 , мы получаем, что

$$
\widetilde{\varphi}_{\mu_{+}}\left(\left(T_{\alpha_{1}} T_{\beta_{1}}\right)^{3}\right)=0, \quad \widetilde{\varphi}_{\mu_{-}}\left(\left(T_{\alpha_{1}} T_{\beta_{1}}\right)^{3}\right)=2 .
$$

Рассмотрим дополнение $\Sigma \backslash \boldsymbol{m}$; это открытая поверхность рода $g-1$ с двумя “дырками”. Обозначим через $\Sigma^{\circ}$ пополнение поверхности $\Sigma \backslash \boldsymbol{m}$, получающееся добавлением двух граничных окружностей $\partial_{1}, \partial_{2}$, каждая из которых естественным образом отождествляется с $\boldsymbol{m}$. Тогда $\Sigma^{\circ}$ - это компакная поверхность с краем, внутренность которой есть $\Sigma \backslash \boldsymbol{m}$. Включение $\Sigma \backslash \boldsymbol{m} \hookrightarrow \Sigma$ продолжается по непрерывности до отображения $\Sigma^{\circ} \rightarrow \Sigma$, при котором каждая из граничных окружностей $\partial_{1}, \partial_{2}$ переходит в $\boldsymbol{m}$. В дальнейшем обозначение $\Sigma^{\circ}$ будет использоваться и для поверхности $\Sigma \backslash \boldsymbol{m}$. Помимо этого, мы будем называть отображение $\Sigma^{\circ} \rightarrow \Sigma$ включением, что, строго говоря, некорректно. Будем надеяться, что читатель не будет введен в заблуждение этими вольностями речи.

Включение $\Sigma^{\circ} \rightarrow \Sigma$ индуцирует гомоморфизм $\operatorname{Map}\left(\Sigma^{\circ}, z_{0}\right) \rightarrow \operatorname{Map}\left(\Sigma, z_{0}\right)$. Отсюда мы получаем действие группы $\operatorname{Map}\left(\Sigma^{\circ}, z_{0}\right)$ на группе гомологий $\mathrm{H}_{1}\left(\Sigma, \mathbb{Z}_{2}\right)$. Для класса $\lambda \in \mathrm{H}_{1}\left(\Sigma, \mathbb{Z}_{2}\right)$ обозначим через $\operatorname{Map}\left(\Sigma^{\circ}, z_{0}\right)_{\lambda}$ его стабилизатор и через $\widetilde{\varphi}_{\lambda}: \operatorname{Map}\left(\Sigma^{\circ}, z_{0}\right)_{\lambda} \rightarrow \mathbb{Z}_{4}$ композицию гомоморфизмов $\operatorname{Map}\left(\Sigma^{\circ}, z_{0}\right)_{\lambda} \rightarrow$ $\operatorname{Map}\left(\Sigma, z_{0}\right)_{\lambda}$ и $\widetilde{\varphi}_{\lambda}: \operatorname{Map}\left(\Sigma, z_{0}\right)_{\lambda} \rightarrow \mathbb{Z}_{4}$. Эти обозначения будут использоваться при $\lambda=\mu_{ \pm}$.

Заметим, что по построению геометрическая реализация монодромии $\widehat{F}_{\Gamma}^{\prime}$ действует на поверхности $\Sigma^{\circ}$, оставляя ее границу неподвижной. Это означает, что монодромия $\widehat{F}_{\Gamma}^{\prime}$, рассматриваемая как элемент группы $\operatorname{Map}_{g, 1}=\operatorname{Map}\left(\Sigma, z_{0}\right)$, лежит в образе гомоморфизма $\operatorname{Map}\left(\Sigma^{\circ}, z_{0}\right) \rightarrow \operatorname{Map}\left(\Sigma, z_{0}\right)$.

Лемма 3.2. Стабилизаторы $\operatorname{Map}\left(\Sigma^{\circ}, z_{0}\right)_{\mu_{+}}$, $\operatorname{Map}\left(\Sigma^{\circ}, z_{0}\right)_{\mu_{-}}$и гомоморфизмыс $\widetilde{\varphi}_{\mu_{ \pm}}: \operatorname{Map}\left(\Sigma^{\circ}, z_{0}\right)_{\mu_{ \pm}} \rightarrow \mathbb{Z}_{4}$ совпадают. В частности, $\widetilde{\varphi}_{\mu_{+}}\left(\widehat{F}_{\Gamma}^{\prime}\right) \equiv \widetilde{\varphi}_{\mu_{-}}\left(\widehat{F}_{\Gamma}^{\prime}\right)$.

Мы докажем лемму 3.2 при более общем предположении, что $\mu_{+}, \mu_{-} \in$ $\mathrm{H}_{1}\left(\Sigma, \mathbb{Z}_{2}\right)$ - произвольные ненулевые классы гомологий, удовлетворяющие условию $\mu_{+}+\mu_{-}=\mu$. В частности, соотношение $\widetilde{\varphi}_{\mu_{+}}\left(\widehat{F}_{\Gamma}^{\prime}\right) \equiv \widetilde{\varphi}_{\mu_{-}}\left(\widehat{F}_{\Gamma}^{\prime}\right)$ будет доказано и в случае, когда $\mu_{ \pm} \cap \mu \equiv 1 \bmod 2$.

ДоКАЗАТЕЛЬСТВо ЛЕммы 3.2. Поясним основную идею доказательства. Напомним, что согласно предложению 2.29 для любого $\lambda \neq 0 \in \mathrm{H}_{1}\left(\Sigma, \mathbb{Z}_{2}\right)$ его стабилизатор $\operatorname{Map}_{g, 1, \lambda}$ и гомоморфизм $\widetilde{\varphi}_{\lambda}: \operatorname{Map}_{g, 1, \lambda} \rightarrow \mathbb{Z}_{4}$ определены в терминах $\lambda$. А именно, образующие группы Map $_{g, 1, \lambda}$ есть либо скручивания Дена $T_{\delta}$ при $\delta \cap \lambda \equiv 0 \bmod 2$, для которых мы полагаем $\widetilde{\varphi}_{\lambda}\left(T_{\delta}\right)=0$, либо квадраты скручиваний Дена $T_{\delta}^{2}$ при $\delta \cap \lambda \equiv 1 \bmod 2$, для которых $\widetilde{\varphi}_{\lambda}\left(T_{\delta}^{2}\right)=1$. В ходе доказательства мы покажем, что:

i) группы $\operatorname{Map}\left(\Sigma^{\circ}, z_{0}\right)_{\mu_{ \pm}}$и гомоморфизмы $\widetilde{\varphi}_{\mu_{ \pm}}: \operatorname{Map}\left(\Sigma^{\circ}, z_{0}\right)_{\mu_{ \pm}} \rightarrow \mathbb{Z}_{4}$ допускают аналогичное описание с несколько более сложным множеством образующих;

ii) имеется общее множество образующих для обеих групп $\operatorname{Map}\left(\Sigma^{\circ}, z_{0}\right)_{\mu_{ \pm}}$, a значения гомоморфизмов $\widetilde{\varphi}_{\mu_{ \pm}}$на каждой из этих образующих равны.

Грубо говоря, эти свойства имеют место в силу того факта, что $\mu_{+} \cap \delta \equiv$ $\mu_{-} \cap \delta \bmod 2$ для любой кривой $\delta$ на поверхности $\Sigma^{\circ}$. 
Известно, что группа классов отображений $\operatorname{Map}_{h, k,[l]}$ поверхности рода $h \geqslant 1$ порождается скручиваниями Дена $T_{\delta}$ относительно неразбивающих кривых и граничных окружностей (см., например, [28] или [22, теорема В и лемма 5.1 в специальном случае $h=1])$. В нашей ситуации группа $\operatorname{Map}\left(\Sigma^{\circ}, z_{0}\right)$ имеет тип $\operatorname{Map}_{g-1,1,[2]}$, а граничные окружности - это $\partial_{1}$ и $\partial_{2}$. При гомоморфизме $\operatorname{Map}\left(\Sigma^{\circ}, z_{0}\right) \rightarrow \operatorname{Map}\left(\Sigma, z_{0}\right)$ оба скручивания Дена $T_{\partial_{1}}$ и $T_{\partial_{2}}$ переходят в скручивание $T_{\boldsymbol{m}}$ относительно окружности $\boldsymbol{m}$, которая не разбивает поверхность $\Sigma$.

Из равенства $\mu_{+} \cap \delta \equiv \mu_{-} \cap \delta$ немедленно следует соотношение $T_{\delta}\left(\mu_{+}\right)+$ $\mu_{+} \equiv T_{\delta}\left(\mu_{-}\right)+\mu_{-} \in \mathrm{H}_{1}\left(\Sigma, \mathbb{Z}_{2}\right)$ для любой кривой $\delta$, удовлетворяющей этому условию. Поскольку группа $\operatorname{Map}\left(\Sigma^{\circ}, z_{0}\right)$ порождена скручиваниями Дена $T_{\delta}$, мы заключаем, что для любого элемента $f \in \operatorname{Map}\left(\Sigma^{\circ}, z_{0}\right)$ выполнено $f\left(\mu_{-}\right)=f\left(\mu_{+}\right)+\mu_{-}+\mu_{+}=f\left(\mu_{+}\right)+\mu$. В частности, формула $\lambda \mapsto \lambda+\mu$ задает $\operatorname{Map}\left(\Sigma^{\circ}, z_{0}\right)$-эквивариантную биекцию между $\operatorname{Map}\left(\Sigma^{\circ}, z_{0}\right)$-орбитами классов $\mu_{+}$и $\mu_{-}$в группе $\mathrm{H}_{1}\left(\Sigma, \mathbb{Z}_{2}\right)$. Следовательно, стабилизаторы $\operatorname{Map}\left(\Sigma^{\circ}, z_{0}\right)_{\mu_{+}}$ и $\operatorname{Map}\left(\Sigma^{\circ}, z_{0}\right)_{\mu_{-}}$действительно совпадают.

Пусть $H$ - образ группы $\operatorname{Map}\left(\Sigma^{\circ}, z_{0}\right)$ в $\boldsymbol{S p}\left(2 g, \mathbb{Z}_{2}\right)$, и пусть $H_{\mu_{ \pm}}$- общий стабилизатор классов $\mu_{+}$и $\mu_{-}$в $H$. Мы утверждаем, что $H_{\mu_{ \pm}}$порождается образами скручиваний $T_{\delta}$ относительно окружности $\boldsymbol{m}$ и неразбивающих кривых $\delta$ на поверхности $\Sigma^{\circ}$, удовлетворяющих условию $\delta \cap \mu_{ \pm} \equiv 0 \bmod 2$. Структура группы $H_{\mu_{ \pm}}$немного различается в случаях, когда $\mu_{ \pm} \cap \mu \equiv 0 \bmod 2$ или $\mu_{ \pm} \cap \mu \equiv 1 \bmod 2$. В обоих случаях группа $H_{\mu_{ \pm}}$тривиально действует на $\mathbb{Z}_{2}$-плоскости $\mathbb{Z}_{2}\left\langle\mu, \mu_{+}\right\rangle \subset \mathrm{H}_{1}\left(\Sigma, \mathbb{Z}_{2}\right)$. Эта плоскость симплектическая относительно $\mathbb{Z}_{2}$-формы пересечения $\cap$ на $\mathrm{H}_{1}\left(\Sigma, \mathbb{Z}_{2}\right)$, если $\mu \cap \mu_{ \pm} \equiv 1 \bmod 2$, и изотропная в противном случае. В симплектическом случае $H_{\mu_{ \pm}}$есть в точности группа $\boldsymbol{S} \boldsymbol{p}\left(2 g-2, \mathbb{Z}_{2}\right)$, действующая симплектически на $\cap$-ортогональном $\mathbb{Z}_{2}$-пространстве $\mathbb{Z}_{2}\left\langle\mu, \mu_{+}\right\rangle^{\perp} \subset \mathrm{H}_{1}\left(\Sigma, \mathbb{Z}_{2}\right)$. Значит, $H_{\mu_{ \pm}}$порождается элементами указанного вида.

В изотропном случае $\mathbb{Z}_{2}$-пространство $\mathbb{Z}_{2}\left\langle\mu, \mu_{+}\right\rangle^{\perp} \subset \mathrm{H}_{1}\left(\Sigma, \mathbb{Z}_{2}\right)$, являющееся $\cap$-ортогональным, содержит плоскость $\mathbb{Z}_{2}\left\langle\mu, \mu_{+}\right\rangle$, и факторпространство $\mathbb{Z}_{2}\left\langle\mu, \mu_{+}\right\rangle^{\perp} / \mathbb{Z}_{2}\left\langle\mu, \mu_{+}\right\rangle$есть снова симплектическое $\mathbb{Z}_{2}$-пространство размерности $2 g-4$. В этом случае группа $H_{\mu_{ \pm}}$сохраняет флаг векторных подпространств $\mathbb{Z}_{2}\left\langle\mu, \mu_{+}\right\rangle \subset \mathbb{Z}_{2}\left\langle\mu, \mu_{+}\right\rangle^{\perp} \subset \mathrm{H}_{1}\left(\Sigma, \mathbb{Z}_{2}\right)$, и потому $H_{\mu_{ \pm}}$состоит из блочных матриц вида $\left(\begin{array}{ccc}\text { id } & B & C \\ 0 & A & B^{t} \\ 0 & 0 & \text { id }\end{array}\right)$, где $A$ лежит в $\boldsymbol{S p}\left(2 g-4, \mathbb{Z}_{2}\right)$ и действует симплектически на $\mathbb{Z}_{2}\left\langle\mu, \mu_{+}\right\rangle^{\perp} / \mathbb{Z}_{2}\left\langle\mu, \mu_{+}\right\rangle, B$ - произвольная $(2 \times(2 g-4))$-матрица с элементами из $\mathbb{Z}_{2}$, а $C$ - симметрическая $(2 \times 2)$-матрица с элементами из $\mathbb{Z}_{2}$. В специальном случае $g=2$ пространство $\mathbb{Z}_{2}\left\langle\mu, \mu_{+}\right\rangle^{\perp} / \mathbb{Z}_{2}\left\langle\mu, \mu_{+}\right\rangle$нулевое и блоки $A, B, B^{t}$ пустые. Теперь утверждение об образующих группы $H_{\mu_{ \pm}}$сводится к простому упражнению по линейной алгебре.

Пусть теперь $x$ - произвольный элемент из стабилизатора $\operatorname{Map}\left(\Sigma^{\circ}, z_{0}\right) \mu_{ \pm}$. Как было показано выше, элемент $x$ может быть представлен в виде произведения скручиваний Дена $\prod_{j} T_{\delta_{j}}^{\epsilon_{j}}$ относительно кривых $\delta_{j}$ на поверхности $\Sigma^{\circ}$, удовлетворяющих следующему условию:

$(\delta)$ каждая кривая $\delta_{j}$ либо не разбивает поверхность $\Sigma^{\circ}$, либо является одной из граничных окружностей $\partial_{1}, \partial_{2}$.

В силу предыдущего рассуждения существует другое разложение на множители $x^{\prime}=\prod_{i} T_{\delta_{i}^{\prime}}^{\epsilon^{\prime}}$, удовлетворяющее условию $(\delta)$ и такое, что $x$ и $x^{\prime}$ имеют 
один и тот же образ в группе $\boldsymbol{S p}\left(2 g, \mathbb{Z}_{2}\right)$, и каждая кривая $\delta_{i}^{\prime}$ отображается в $H_{\mu_{ \pm}} \subset \boldsymbol{S} \boldsymbol{p}\left(2 g, \mathbb{Z}_{2}\right)$. Тогда $x^{\prime}$ лежит в группе $\operatorname{Map}\left(\Sigma^{\circ}, z_{0}\right)_{\mu_{ \pm}}$и оба гомоморфизма $\widetilde{\varphi}_{\mu_{+}}, \widetilde{\varphi}_{\mu_{-}}$принимают на этом элементе значение нуль. Заменяя $x$ на $x \cdot\left(x^{\prime}\right)^{-1}$, мы сводим лемму к специальному случаю, когда $x$ имеет тривиальную проекцию в группу $\boldsymbol{S} \boldsymbol{p}\left(2 g, \mathbb{Z}_{2}\right)$ и по-прежнему допускает разложение на множители $x=\prod_{j} T_{\delta_{j}}^{\epsilon_{j}}$, удовлетворяющее условию $(\delta)$.

При этом дополнительном предположении предложение 2.22 дает алгоритм для преобразования разложения $x=\prod_{j} T_{\delta_{j}}^{\epsilon_{j}}$ в разложение вида $x=\prod_{i}\left(T_{\delta_{i}}^{2}\right)^{\epsilon_{i}}$, содержащее только квадраты скручиваний Дена. В этом алгоритме мы воспользуемся геометрическим базисом $\alpha_{1}, \ldots, \alpha_{g} ; \beta_{1}, \ldots, \beta_{g}$ поверхности $\Sigma$, выбранным в начале настоящего параграфа. Для этого базиса $\mu=\left[\beta_{1}\right]_{\mathbb{Z}_{2}}$. Мы утверждаем, что в этом случае шаги 1-9 из доказательства предложения 2.22 могут быть сделаны так, что условие $(\delta)$ будет выполнено для всех участвующих в рассуждении скручиваний Дена $T_{\delta}$. Чтобы в этом убедиться, заметим, что мы применяем следующие три операции:

(i) движения Гурвица;

(ii) вставка сомножителя вида $T_{\beta} T_{\beta}^{-1}$;

(iii) вставка фонарного элемента $L$.

Операция (i) является сопряжением и, значит, не разрушает свойство $(\delta)$. Условия, налагаемые на кривые, участвующие в операциях (ii) и (iii), касаются только их $\mathbb{Z}_{2}$-гомологических классов, поэтому всегда можно найти подходящую кривую $\beta$ или, соответственно, фонарную конфигурацию кривых.

Выполнив шаг 9 , мы получаем для элемента $x$ разложение вида $\prod_{i}\left(T_{\gamma_{i}}^{2}\right)^{\epsilon_{i}} \times$ $\prod_{j}\left(T_{\delta_{j}} T_{\delta_{j}^{\prime}}^{-1}\right)$, где кривые $\gamma_{i}, \delta_{j}, \delta_{j}^{\prime}$ удовлетворяют условию $(\delta)$, и $\left[\delta_{j}\right]=\left[\delta_{j}^{\prime}\right] \in$ $\mathrm{H}_{1}\left(\Sigma, \mathbb{Z}_{2}\right)$. Гомоморфизмы $\widetilde{\varphi}_{\mu_{ \pm}}$принимают одинаковые значения на каждом сомножителе $T_{\gamma_{i}}^{2}$. Поэтому достаточно рассмотреть специальный случай, когда $x=T_{\delta} T_{\delta^{\prime}}^{-1}$ для некоторых кривых $\delta, \delta^{\prime}$, удовлетворяющих условию $(\delta)$ и имеющих один и тот же класс $\mathbb{Z}_{2}$-гомологий на поверхности $\Sigma$. Случай, когда $\delta, \delta^{\prime}$ - это граничные окружности $\partial_{1}, \partial_{2}$, тривиален, поскольку обе эти окружности соответствуют кривой $\boldsymbol{m}$ на $\Sigma$. Таким образом, мы можем предполагать, что $\delta$ и $\delta^{\prime}$ являются неразбивающими кривыми на $\Sigma^{\circ}$. В частности, они представляют ненулевой примитивный класс гомологий.

Шаг 10 из доказательства предложения 2.22 показывает, как преобразовать элемент вида $x=T_{\delta} T_{\delta^{\prime}}^{-1}$, где $[\delta]_{\mathbb{Z}_{2}}=\left[\delta^{\prime}\right]_{\mathbb{Z}_{2}} \in \mathrm{H}_{1}\left(\Sigma, \mathbb{Z}_{2}\right)$, в разложение $\prod_{i}\left(T_{\gamma_{i}}^{2}\right)^{\epsilon_{i}} \cdot \prod_{j}\left(T_{\delta_{j}} T_{\delta_{j}^{\prime}}^{-1}\right)$ такого же типа, как и выше, но с дополнительным равенством $\left[\delta_{j}\right]=\left[\delta_{j}^{\prime}\right]$ иелочисленных гомологических классов. Мы утверждаем, что в нашей ситуации это можно сделать, не нарушив условия $(\delta)$. Действительно, шаг 10 преобразует заданный элемент $x=T_{\delta} T_{\delta^{\prime}}^{-1}$ с помощью вставки произведений $T_{\gamma_{i}}^{-2} T_{\gamma_{i}}^{2}$ и сопряжения $T_{\delta^{\prime}}$ с помощью $T_{\gamma_{i}}^{2}$; при этом сомножители $T_{\gamma_{i}}^{-2}$ и $T_{\gamma_{i}}^{2}$ также сопрягаются. Условие, наложенное на участвующие в этом преобразовании кривые $\gamma_{i}$, есть $\left[\gamma_{i}\right] \cap\left[\delta_{i}\right]=1$. Поскольку кривые $\delta$ и $\delta^{\prime}$ не разбивают поверхность $\Sigma^{\circ}$, существуют такие кривые $\gamma_{i}$, удовлетворяющие также и условию $(\delta)$. Отсюда следует, что шаг 10 можно выполнить, сохраняя условие $(\delta)$.

В качестве примера покажем, как изменить целочисленный класс гомологий кривой $\delta^{\prime}$ на $2[\boldsymbol{m}]$. Выберем вложенную кривую $\gamma$ на поверхности $\Sigma^{\circ}$, 
удовлетворяющую условию $[\gamma] \cap\left[\delta^{\prime}\right]=1$, а также вложенную кривую $\gamma^{\prime}$, представляющую класс гомологий $[\gamma]+[\boldsymbol{m}]$. Тогда

$$
\begin{aligned}
T_{\delta} T_{\delta^{\prime}}^{-1} & =T_{\delta} \cdot T_{\gamma}^{2} T_{\gamma^{\prime}}^{-2} T_{\gamma^{\prime}}^{2} T_{\gamma}^{-2} \cdot T_{\delta^{\prime}}^{-1}=T_{\delta} T_{\gamma}^{2} T_{\gamma^{\prime}}^{-2} \cdot T_{\delta^{\prime \prime}}^{-1} \cdot T_{\gamma^{\prime}}^{2} T_{\gamma}^{-2} \\
& =T_{\delta} \cdot T_{\delta^{\prime \prime}}^{-1} \cdot T_{\gamma^{*}}^{2} T_{\left(\gamma^{\prime}\right)^{*}}^{-2} \cdot T_{\gamma^{\prime}}^{2} T_{\gamma}^{-2}
\end{aligned}
$$

где все преобразования суть движения Гурвица. Поскольку $T_{\alpha} T_{\beta} T_{\alpha}^{-1}=T_{T_{\alpha}(\beta)}$, мы получаем, что $\delta^{\prime \prime}=T_{\gamma^{\prime}}^{2} T_{\gamma}^{-2}\left(\delta^{\prime}\right), \gamma^{*}=T_{\delta^{\prime \prime}}(\gamma)$ и $\left(\gamma^{\prime}\right)^{*}=T_{\delta^{\prime \prime}}\left(\gamma^{\prime}\right)$. В частности,

$$
\left[\delta^{\prime \prime}\right]=\left[\delta^{\prime}\right]-2[\gamma]+2[\gamma+\boldsymbol{m}]=\left[\delta^{\prime}\right]+2[\boldsymbol{m}],
$$

что и требовалось доказать.

Наконец, рассмотрим преобразования, выполняемые на шаге 11 и шаге 12 из доказательства предложения 2.22. Там доказывается, что две неразбивающие кривые $\delta, \delta^{\prime}$ на поверхности $\Sigma$, представляющие один и тот же целочисленный гомологический класс, отличаются на элемент $y$, лежащий в группе Торелли $\mathcal{I}_{g}$ поверхности $\Sigma$. Это означает, что элемент $T_{\delta} T_{\delta^{\prime}}^{-1}$ является коммутатором $\left[T_{\delta}, y\right]$. Из соотношения

$$
[x, y \cdot z]=[x, y] \cdot y[x, z] y^{-1}=[x, y] \cdot\left[y x y^{-1}, y z y^{-1}\right]
$$

следует, что достаточно рассматривать только коммутаторы $\left[T_{\delta}, y\right]$, в которых элемент $y$ пробегает подходящее множество образующих группы $\mathcal{I}_{g}$.

Случай $g=2$ является особым, поскольку образующие группы $\mathcal{I}_{2}$ имеют другой вид, чем в случае $g \geqslant 3$. Воспользуемся многочисленными изоморфизмами, имеющими место для поверхностей рода один и два: $\mathcal{S}_{1}=\mathrm{A}_{2}$, $\operatorname{Map}_{1,[1]}=\operatorname{Br}\left(\mathrm{A}_{2}\right)=\mathrm{Br}_{3}, \boldsymbol{S l}\left(2, \mathbb{Z}_{2}\right)=\mathrm{W}\left(\mathrm{A}_{2}\right)=\mathrm{Sym}_{3}, \mathcal{T}\left(\mathrm{A}_{2}\right)=\mathcal{T}\left(\mathcal{S}_{1}\right)=\mathcal{H}_{1}$

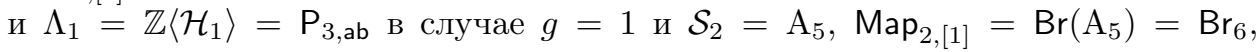
$\boldsymbol{S p}\left(4, \mathbb{Z}_{2}\right)=\mathrm{W}\left(\mathrm{A}_{5}\right)=\operatorname{Sym}_{6}, \mathcal{T}\left(\mathrm{A}_{5}\right)=\mathcal{T}\left(\mathcal{S}_{2}\right)=\mathcal{H}_{2}$ и $\Lambda_{2}=\mathbb{Z}\left\langle\mathcal{H}_{2}\right\rangle=\mathrm{P}_{6, \text { aь в }}$ случае $g=2$. Группа $\mathcal{I}_{2}$ порождается скручиваниями Дена относительно кривых $\gamma$, разбивающих поверхность $\Sigma$ на две части $\Sigma^{\prime}, \Sigma^{\prime \prime}$, каждая из которых есть тор с дыркой (см. [40] или [30]). Пусть $\alpha, \beta$ - геометрический базис поверхности $\Sigma^{\prime}$. Тогда группа $\operatorname{Map}\left(\Sigma^{\prime}\right)$ изоморфна группе кос $\mathrm{Br}_{3}$ со стандартными образующими $T_{\alpha}, T_{\beta}$, а $T_{\gamma}=\left(T_{\alpha} T_{\beta}\right)^{6}$ (см. теорему 2.19). Более того, произведение $\left(T_{\alpha} T_{\beta}\right)^{6}$ равно $\Delta^{4}\left(\mathrm{Br}_{3}\right)$. Значит, $T_{\gamma}=\Delta^{4}\left(\mathrm{Br}_{3}\right)$ лежит в группе крашеных кос $\mathrm{P}_{3}$, и в силу леммы 2.16 проекция элемента $T_{\gamma}$ в абелианизацию $\mathrm{P}_{3, \mathrm{ab}}=\mathbb{Z}\left\langle\mathcal{H}_{1}\right\rangle$ равна $2 A_{[\alpha]}+2 A_{[\beta]}+2 A_{[\alpha]+[\beta]}$. Здесь через $[\alpha]$ и $[\beta]$ обозначены $\mathbb{Z}_{2}$-гомологические классы, так что $[\alpha],[\beta],[\alpha]+[\beta]$ исчерпывают множество $\mathcal{H}_{1}$ нетривиальных элементов в группе $\mathrm{H}_{1}\left(\Sigma^{\prime}, \mathbb{Z}_{2}\right)$. Следовательно, коммутатор $\left[T_{\delta}, T_{\gamma}\right]$ также лежит в группе крашеных кос, а его проекция в группу $\mathrm{P}_{6, \text { ab }}=\mathbb{Z}\left\langle\mathcal{H}_{2}\right\rangle$ равна

$$
2 A_{[\alpha]}+2 A_{[\beta]}+2 A_{[\alpha]+[\beta]}-2 A_{\left[T_{\delta}(\alpha)\right]}+2 A_{\left[T_{\delta}(\beta)\right]}+2 A_{\left[T_{\delta}(\alpha)\right]+\left[T_{\delta}(\beta)\right]} .
$$

Класс $\mathbb{Z}_{2}$-гомологий $\left[T_{\delta}(\alpha)\right]$ равен $[\alpha]+[\delta]$ в случае $\alpha \cap \delta \equiv 1 \bmod 2$ и равен $[\alpha]$ в случае $\alpha \cap \delta \equiv 0 \bmod 2$. Следовательно, значение $\widetilde{\varphi}_{\lambda}\left(2 A_{[\alpha]}-2 A_{\left[T_{\delta}(\alpha)\right]}\right)$ равно $2 \bmod 4$, если $\alpha \cap \delta \equiv \lambda \cap \delta \equiv 1 \bmod 2$, и нулю в противном случае. Поскольку в нашей ситуации $\mu_{+} \cap \delta \equiv \mu_{-} \cap \delta \bmod 2$, гомоморфизмы $\widetilde{\varphi}_{\mu_{+}}$и $\widetilde{\varphi}_{\mu_{-}}$принимают одинаковые значения на всех коммутаторах $\left[T_{\delta}, T_{\gamma}\right]$ рассматриваемого вида. 
Теперь рассмотрим случай коммутаторов вида $\left[T_{\delta}, x\right]$, где $x$ - это образующая группы Торелли $\mathcal{I}_{g}$ при $g \geqslant 3$. Как было показано на шаге 12 в доказательстве предложения 2.22 (см. формулу (2.6) и рассуждение непосредственно после нее), в этом случае группа $\mathcal{I}_{g}$ порождается элементами вида $T_{\eta}^{2}\left(T_{\beta} T_{\alpha} T_{\beta^{\prime}}\right)^{-4}$, где кривые $\eta, \beta, \alpha, \beta^{\prime}$ лежат в некоторой неразбивающей цепной конфигурации $\mathcal{C}$ (см. рис. 1$)$. Заметим, что $\left(T_{\beta} T_{\alpha} T_{\beta^{\prime}}\right)^{4}$ есть квадрат элемента Гарсайда $\Delta^{2}\left(\mathrm{~A}_{3}\right)$ системы Кокстера $\left\{T_{\beta}, T_{\alpha}, T_{\beta^{\prime}}\right\} \cong \mathrm{A}_{3}$. Значит, $\Delta^{2}\left(\mathrm{~A}_{3}\right)$ лежит в группе крашеных кос $\mathrm{P}\left(\mathrm{A}_{3}\right)$, а его проекция в группу $\mathrm{P}_{\mathrm{ab}}\left(\mathrm{A}_{3}\right)$ равна сумме $\sum_{t \in \mathcal{T}\left(\mathrm{A}_{3}\right)} A_{t}$ (см. лемму 2.16). С точностью до сопряжения мы можем считать, что кривые $\beta, \alpha, \beta^{\prime}$ есть кривые $a_{1}, a_{2}, a_{3}$ из системы $\mathcal{S}_{g}$ (см. рис. 3). В свою очередь, $\left\{a_{1}, a_{2}, a_{3}\right\}$ есть подсистема в $\mathrm{A}_{5}=\left\{a_{1}, \ldots, a_{4}, a_{0}\right\}$. Таким образом, $\mathcal{T}\left(\mathrm{A}_{3}\right)$ есть подмножество в $\mathcal{T}\left(\mathrm{A}_{5}\right)=\mathcal{H}\left(\mathcal{S}_{2}\right)$. Прямое вычисление показывает, что $\mathcal{T}\left(\mathrm{A}_{3}\right)$ состоит из следующих $\mathbb{Z}_{2}$-гомологических классов: $\left[a_{1}\right],\left[a_{2}\right],\left[a_{3}\right],\left[a_{1}\right]+\left[a_{2}\right],\left[a_{2}\right]+\left[a_{3}\right],\left[a_{1}\right]+\left[a_{2}\right]+\left[a_{3}\right]$, т. е. всех ненулевых векторов в векторном пространстве над $\mathbb{Z}_{2}$, порожденном классами $\left[a_{1}\right]=[\beta],\left[a_{2}\right]=[\alpha],\left[a_{3}\right]=\left[\beta^{\prime}\right]$, за исключением $\left[a_{1}\right]+\left[a_{3}\right]$. Обозначим через $V$ это $\mathbb{Z}_{2}$-векторное пространство, а через $V^{\prime}$ - подмножество $V \backslash\{0\}$. Заметим еще, что $\left[a_{1}\right]+\left[a_{3}\right]=[\beta]+\left[\beta^{\prime}\right]=[\eta]$. Отсюда мы получаем, что проекция образующей $T_{\eta}^{2}\left(T_{\beta} T_{\alpha} T_{\beta^{\prime}}\right)^{-4}$ в группу $\mathrm{P}_{\mathrm{ab}}\left(\mathrm{A}_{3}\right)$ равна $2 A_{[\eta]}-\sum_{v \in V^{\prime}} A_{v}$. Далее, для любого класса $\lambda \in \mathrm{H}_{1}\left(\Sigma, \mathbb{Z}_{2}\right)$ индекс пересечения $\lambda \cap v \in \mathbb{Z}_{2}$ либо равен нулю для всех $v \in V$, либо отличен от нуля в точности для четырех элементов $v \in V^{\prime}$. Отсюда следует, что гомоморфизм $\widetilde{\varphi}_{\lambda}$ обращается в нуль на $\sum_{v \in V^{\prime}} A_{v}$ и на образах этого класса под действием любых элементов $y \in$ Map $_{g, 1}$. Поэтому значения гомоморфизмов $\widetilde{\varphi}_{\mu_{+}}$и $\widetilde{\varphi}_{\mu_{-}}$на элементе $\left[T_{\delta}, x\right]$ рассматриваемого здесь вида равны их значениям на $2 A_{[\eta]}-2 A_{\left[T_{\delta}(\eta)\right]}$. Однако выше было показано, что если $\mu_{+} \cap \delta \equiv \mu_{-} \cap \delta \bmod 2$, то значения гомоморфизмов $\widetilde{\varphi}_{\mu_{ \pm}}$на $2 A_{[\eta]}-2 A_{\left[T_{\delta}(\eta)\right]}$ совпадают. Лемма доказана.

Вернемся теперь к доказательству предложения 3.1. Применяя лемму 3.2 и соотношения $(3.1)$ к рассмотренному выше разложению $\widehat{F}_{\Gamma}=\widehat{F}_{\Gamma}^{\prime} \cdot\left(T_{\alpha_{1}} T_{\beta_{1}}\right)^{3}$, мы получаем, что

$$
\widetilde{\varphi}_{\mu_{+}}\left(\widehat{F}_{\Gamma}\right)-\widetilde{\varphi}_{\mu_{-}}\left(\widehat{F}_{\Gamma}\right) \equiv 2 \bmod 4 \text {. }
$$

Пусть $F_{+}$и $F_{-}-$разложения монодромии $F_{\Gamma}$ вида $F_{ \pm}:=\prod_{i}\left[F_{2 i-1}, F_{2 i}\right] \cdot \prod_{j} T_{\delta_{j}}$, получающиеся из гомоморфизмов монодромии $\mathcal{F}_{ \pm}: \pi_{1}\left(Y_{ \pm}^{\circ}\right) \rightarrow \mathrm{Map}_{g}$. Поднимая гомоморфизмы $\mathcal{F}_{ \pm}$в группу $\mathrm{Map}_{g, 1}$, мы получаем подъемы $\widehat{F}_{ \pm} \in$ Map $_{g, 1}$ монодромии $F_{\Gamma}$, которые отличаются от $\widehat{F}_{\Gamma}$ на элементы, лежащие в образе гомоморфизма $\partial_{\pi}: \pi_{1}(\Sigma) \rightarrow$ Map $_{g, 1}$.

Предположим, что род $g$ нечетен. Тогда гомоморфизмы $\widetilde{\varphi}_{\mu}, \widetilde{\varphi}_{\mu_{ \pm}}$обращаются в нуль по модулю 4 на образе гомоморфизма $\partial_{\pi}$ в силу предложения $2.29,(\varphi 3)$. Значит, $\widetilde{\varphi}_{\mu_{+}}\left(\widehat{F}_{\Gamma}\right) \equiv \widetilde{\varphi}_{\mu_{+}}\left(\widehat{F}_{+}\right) \bmod 4$. Напомним, что элемент $\widehat{F}_{+}$был построен вместе с разложением $\widehat{F}_{+}=\prod_{i}\left[\widehat{F}_{2 i-1}, \widehat{F}_{2 i}\right] \cdot \prod_{j} T_{\delta_{j}}$, в котором $\delta_{j} \cap \mu_{+} \equiv 0 \bmod 2$, и что класс $\mu_{+}$инвариантен относительно $\widehat{F}_{i}$. Поэтому гомоморфизм $\widetilde{\varphi}_{\mu_{+}}$обращается в нуль на каждом скручивании $T_{\delta_{j}}$ по предложению $2.29,(\varphi 1)$. Далее, инвариантность $\mu_{+}$относительно преобразований $\widehat{F}_{i}$ означает, что $\widehat{F}_{i}$ лежат в подгруппе $\operatorname{Map}_{g, 1, \mu_{+}}$. Следовательно, $\widetilde{\varphi}_{\mu_{+}}$обращается в нуль на всех 
коммутаторах $\left[\widehat{F}_{2 i-1}, \widehat{F}_{2 i}\right]$. Итак, мы получаем равенство $\widetilde{\varphi}_{\mu_{+}}\left(\widehat{F}_{\Gamma}\right) \equiv 0 \bmod 4$. Аналогично, $\widetilde{\varphi}_{\mu_{-}}\left(\widehat{F}_{\Gamma}\right) \equiv 0 \bmod 4$. Это противоречит соотношению $(3.2)$.

Предположим, что род $g$ четен. Определим $\gamma_{ \pm} \in \pi_{1}(\Sigma)$ из соотношений $\widehat{F}_{ \pm}=\widehat{F}_{\Gamma} \cdot \partial_{\pi}\left(\gamma_{ \pm}\right)$. По теореме 2.30 существует подъем $\widetilde{\mathcal{F}}: \pi_{1}\left(Y^{\circ}\right) \rightarrow$ $\widetilde{\operatorname{Map}}_{g}$ гомоморфизма монодромии $\mathcal{F}: \pi_{1}\left(Y^{\circ}\right) \rightarrow \operatorname{Map}_{g}$ (см. п. 2.3). По лемме 1.12 любой такой подъем $\widetilde{\mathcal{F}}$ соответствует $\mathbb{Z}_{2}$-сечению $\sigma$. Можно считать, что подъемы $\widehat{F}_{ \pm} \in \operatorname{Map}_{g, 1}$ монодромии $F_{\Gamma}$ согласованы с сечением $\sigma$. (Это означает, что проекции элементов $\widehat{F}_{ \pm}$в группу $\widetilde{\operatorname{Map}}_{g}$ совпадают.) Следовательно, $\left[\gamma_{+}\right]=\left[\gamma_{-}\right] \in \mathrm{H}_{1}\left(\Sigma, \mathbb{Z}_{2}\right)$. Сравнивая значения гомоморфизмов $\widetilde{\varphi}_{\mu}$ и $\widetilde{\varphi}_{\mu_{ \pm}}$и воспользовавшись формулой $\widetilde{\varphi}_{\mu_{ \pm}}\left(\widehat{F}_{ \pm}\right)=0$, мы получаем соотношение $\widetilde{\varphi}_{\mu_{+}}\left(\partial_{\pi}\left(\gamma_{+}\right)\right)-\widetilde{\varphi}_{\mu_{-}}\left(\partial_{\pi}\left(\gamma_{-}\right)\right) \equiv 2 \bmod 4$. Теперь, используя предложение $2.29,(\varphi 3)$, мы заключаем, что $\left[\gamma_{+}\right] \cap \mu \equiv 1 \bmod 2$. Это означает, что $[K] \cap[\sigma] \not \equiv 0$. Получено противоречие с условиями предложения.

Допустим теперь, что класс $\mu$ инвариантен при действии группы $\pi_{1}\left(Y_{+}^{\circ}\right)$ преобразованиями монодромии. Поскольку класс $\mu_{+}$также инвариантен при этом действии, это верно и для класса $\mu_{-}=\mu+\mu_{+}$. В частности, $\mu_{+} \cap\left[\delta_{i}\right] \equiv \mu_{-} \cap\left[\delta_{i}\right] \equiv$ $0 \bmod 2$ для любого скручивания Дена $T_{\delta_{i}}$, возникающего в рассмотренном выше разложении монодромии $F_{+}$. Значит, для подъема $\widehat{F}_{+}$разложения $F_{+}$мы получаем соотношение $\widetilde{\varphi}_{\mu_{+}}\left(\widehat{F}_{+}\right) \equiv \widetilde{\varphi}_{\mu_{-}}\left(\widehat{F}_{+}\right) \equiv 0 \bmod 4$.

Следующее рассуждение по существу совпадает с использованным при доказательстве формулы $\mu_{ \pm} \cap \mu \equiv 1 \bmod 2$. А именно, в случае нечетного рода $g$ гомоморфизмы $\widetilde{\varphi}_{\mu_{ \pm}}$обращаются в нуль на образе гомоморфизма $\partial_{\pi}\left(\pi_{1}(\Sigma)\right)$ и, следовательно, $\widetilde{\varphi}_{\mu_{+}}\left(\widehat{F}_{\Gamma}\right) \equiv \widetilde{\varphi}_{\mu_{-}}\left(\widehat{F}_{\Gamma}\right) \equiv 0 \bmod 4$. Это противоречит соотношению (3.2).

В случае четного рода $g$ существует $\mathbb{Z}_{2}$-сечение $\sigma$ проекции $\operatorname{pr}: X \rightarrow Y$ и мы можем предполагать, что подъем $\widehat{F}_{+}$согласован с сечением $\sigma$. Определим $\gamma_{+} \in \pi_{1}(\Sigma)$ из соотношения $\widehat{F}_{+}=\widehat{F}_{\Gamma} \cdot \partial_{\pi}\left(\gamma_{+}\right)$, где $\widehat{F}_{\Gamma}-$ выбранный нами подъем $F_{\Gamma}$. Теперь, используя свойство $(\varphi 3)$ из предложения 2.29 и соотношение $(3.2)$, мы получаем $\varphi_{\mu_{+}}\left(\gamma_{+}\right)-\varphi_{\mu_{-}}\left(\gamma_{+}\right) \equiv 1 \bmod 2$. Поскольку $\varphi_{\mu_{ \pm}}\left(\gamma_{+}\right)$есть просто $\mathbb{Z}_{2}$-индекс пересечения $\mu_{ \pm} \cap \gamma_{+}$, выполнено равенство $\mu \cap \gamma_{+} \equiv 1 \bmod 2$. Итак, $\mathbb{Z}_{2}$-сечение $\sigma$ нетривиально пересекается с $K$, а значит, класс гомологий $[K] \in \mathrm{H}_{2}\left(X, \mathbb{Z}_{2}\right)$ нетривиален. Это противоречит условиям предложения.

Таким образом, класс $\mu$ не может быть инвариантен при действии группы $\pi_{1}\left(Y_{+}^{\circ}\right)$ преобразованиями монодромии. Такое же рассуждение применимо к действию группы $\pi_{1}\left(Y_{-}^{\circ}\right)$.

3.2. Доказательство основной теоремы. Переобозначим через $X_{0}$ исходное линейчатое симплектическое многообразие, а через $X$ обозначим его раздутие, построенное в лемме 1.3 .

Предположим, что утверждение теоремы неверно и бутылка Клейна $K \subset X$ гомологически тривиальна. Перечислим установленные нами в этой ситуации свойства семейства Лефшеца $\mathrm{pr}: X \rightarrow Y$. По лемме 1.3 база $Y$ является сферой $S^{2}$ и окружность $\Gamma$ делит $Y$ на два диска $Y_{ \pm}$. По предложению 1.7 
меридиан $\boldsymbol{m}$ не разбивает слой $\Sigma$, а по предложению 3.1 монодромия семейства pr: $X \rightarrow Y$ имеет следующее свойство: существуют такие классы гомологий $\mu_{+}, \mu_{-} \in \mathrm{H}_{1}\left(\Sigma, \mathbb{Z}_{2}\right)$, что $\mu \cap \mu_{+} \equiv \mu \cap \mu_{-} \equiv 1 \bmod 2$, и монодромия $\mathcal{F}_{ \pm}: \pi_{1}\left(Y_{ \pm}^{\circ}\right) \rightarrow$ Мap $_{g}$ в каждом из дисков $Y_{ \pm}$сохраняет соответствующий класс $\mu_{ \pm}$, но не сохраняет класс $\mu$. Наконец, монодромия $F_{\Gamma}$ вдоль окружности $\Gamma$ может быть реализована отображением, также обозначаемым $F_{\Gamma} \in \mathscr{D}$ iff $f_{+}(\Sigma)$, которое переводит кривую $\boldsymbol{m}$ в себя с обратной ориентацией.

Выберем локальные "полярные" координаты $(r, \varphi)$ в окрестности $U_{\boldsymbol{m}}$ кривой $\boldsymbol{m}$ на поверхности $\Sigma$, в которых $\boldsymbol{m}$ задается уравнением $r=1, \varphi$ является угловой координатой на окружности $\boldsymbol{m}$, а отображение $F_{\Gamma}$ имеет вид $(r, \varphi) \mapsto\left(r^{-1},-\varphi\right)$. Следовательно, имеется геометрическая реализация скручивания Дена $T_{\boldsymbol{m}} \in \mathscr{D}_{\text {iff }}(\Sigma)$ относительно $\boldsymbol{m}$, коммутирующая с диффеоморфизмом $F_{\Gamma}$. Используя это отображение $T_{\boldsymbol{m}}$ и локальную тривиализацию расслоения $\mathrm{pr}: X_{\Gamma} \rightarrow \Gamma$ из определения отображения монодромии $F_{\Gamma}$, мы получаем отображение $\Psi: X_{\Gamma} \rightarrow X_{\Gamma}$, сохраняющее слои и действующее на каждом слое $X_{y}, y \in \Gamma$, как скручивание $T_{\boldsymbol{m}}$.

Построим новое многообразие $X^{\prime}$, склеивая куски $X_{Y_{+}}$и $X_{Y_{-}}$вдоль $X_{\Gamma}=$ $\partial X_{Y_{+}}=\partial X_{Y_{-}}$по отображению $\Psi: X_{\Gamma} \rightarrow X_{\Gamma}$. Очевидно, что на $X^{\prime}$ также имеется семейство Лефшеца $\mathrm{pr}^{\prime}: X^{\prime} \rightarrow Y$ над той же базой $Y=S^{2}$. Более того, монодромия семейства $\mathrm{pr}^{\prime}: X^{\prime} \rightarrow Y$ не изменяется над диском $Y_{+}$и сопрягается отображением $T_{\boldsymbol{m}}$ над диском $Y_{-}$. Поскольку $[\boldsymbol{m}]_{\mathbb{Z}_{2}}=\mu$ и $\mu_{+}=\mu+\mu_{-}$, полная монодромия семейства $\mathrm{pr}^{\prime}: X^{\prime} \rightarrow Y$ сохраняет класс $\mu_{+}$.

Автор благодарен С. Ю. Немировскому за следующее наблюдение. Приведенная выше конструкция многообразия $X^{\prime}$ эквивалентна применению хирургии Люттингера относительно бутылки Клейна $K \subset X$ (см. п. 0.2 и [39]). В свою очередь, данное нами описание показывает, что эта хирургия вдоль бутылки Клейна согласована с проекцией pr семейства Морса-Лефшеца pr: $(X, K) \rightarrow(Y, \Gamma)$ при условии, что проекция pr: $K \rightarrow \Gamma \cong S^{1}$ есть очевидное $S^{1}$-расслоение. Отметим, что такое же согласование имеется и в случае, когда вместо $K$ берется тор $T^{2}$ (см. [6]).

Лемма 3.3. Имеем $\operatorname{rank} \mathrm{H}_{1}\left(X^{\prime}, \mathbb{Z}_{2}\right)=\operatorname{rank} \mathrm{H}_{1}\left(X, \mathbb{Z}_{2}\right)+1$.

ДокАзАТЕЛьство. Пусть $V_{+}$и $V_{-}$- это $\mathbb{Z}_{2}$-подпространства в $\mathrm{H}_{1}\left(\Sigma, \mathbb{Z}_{2}\right)$, порожденные исчезающими классами $\left[\delta_{i}\right]$ семейств pr: $X_{Y_{+}} \rightarrow Y_{+}$и pr: $X_{Y_{-}} \rightarrow$ $Y_{-}$соответственно. Тогда по лемме 1.10 имеем

$$
\begin{gathered}
\mathrm{H}_{1}\left(X, \mathbb{Z}_{2}\right)=\mathrm{H}_{1}\left(\Sigma, \mathbb{Z}_{2}\right) /\left(V_{+}+V_{-}\right), \\
\mathrm{H}_{1}\left(X^{\prime}, \mathbb{Z}_{2}\right)=\mathrm{H}_{1}\left(\Sigma, \mathbb{Z}_{2}\right) /\left(V_{+}+T_{\mu}\left(V_{-}\right)\right) .
\end{gathered}
$$

Положим

$$
W_{0}:=\mathbb{Z}_{2}\left\langle\mu_{+}, \mu_{-}\right\rangle^{\perp} \subset \mathrm{H}_{1}\left(\Sigma, \mathbb{Z}_{2}\right),
$$

так что $\mathrm{H}_{1}\left(\Sigma, \mathbb{Z}_{2}\right)=\mathbb{Z}_{2}\left\langle\mu_{+}, \mu_{-}\right\rangle \oplus W_{0}$ задает ортогональное разложение. По предложению 3.1 фактор $\left(V_{+}+W_{0}\right) / W_{0}$ имеет ранг 1 и порождается классом смежности $\mu_{+}$. Аналогично,

$$
\left(V_{-}+W_{0}\right) / W_{0} \cong \mathbb{Z}_{2}\left\langle\mu_{-}\right\rangle
$$


Мы утверждаем, что класс $\mathbb{Z}_{2}$-гомологий $[\boldsymbol{m}]$ меридиана $\boldsymbol{m}$ в многообразии $X$ тривиален ${ }^{3}$. В силу изоморфизмов (3.3) это утверждение эквивалентно тому, что класс $\mu \in \mathrm{H}_{1}\left(\Sigma, \mathbb{Z}_{2}\right)$ лежит в подпространстве $V_{+}+V_{-}$. Для доказательства рассмотрим гомоморфизм Бокштейна $\boldsymbol{\beta}_{\Sigma}: \mathrm{H}_{2}\left(\Sigma, \mathbb{Z}_{2}\right) \rightarrow \mathrm{H}_{1}\left(\Sigma, \mathbb{Z}_{2}\right)$, соответствующий точной последовательности групп коэффициентов $0 \rightarrow \mathbb{Z}_{2} \rightarrow$ $\mathbb{Z}_{4} \rightarrow \mathbb{Z}_{2} \rightarrow 0$, и аналогичный гомоморфизм в гомологиях многообразия $X$. Поскольку дополнение $K \backslash \boldsymbol{m}$ ориентируемо, оно определяет целочисленную 2 -цепь на $K$, граница которой есть $\pm 2[\boldsymbol{m}]$. Отсюда немедленно следует, что $\boldsymbol{\beta}_{\Sigma}[K]_{\mathbb{Z}_{2}}=\mu \in \mathrm{H}_{1}\left(\Sigma, \mathbb{Z}_{2}\right)$. Теперь требуемое включение $\mu \in V_{+}+V_{-}$ вытекает из согласованности гомоморфизмов Бокштейна с гомоморфизмами $\mathrm{H}_{i}\left(\Sigma, \mathbb{Z}_{2}\right) \rightarrow \mathrm{H}_{i}\left(X, \mathbb{Z}_{2}\right)$.

Итак, мы видим, что существуют векторы $v_{ \pm} \in V_{ \pm}$, для которых $v_{ \pm}-\mu_{ \pm} \in$ $W_{0}$ и $v_{+}-v_{-}-\mu \in W_{0} \cap\left(V_{+}+V_{-}\right)$. Утверждение леммы следует отсюда с помощью элементарной линейной алгебры.

Лемма 3.3 справедлива для любого топологического семейства Лефшеца pr: $X \rightarrow Y$, содержащего вложенную бутылку Клейна $K$, удовлетворяющую условиям (T1), (Т2) из п. 1.4 и дополнительному условию, что кривая $\Gamma=\operatorname{pr}(K)$ разбивает $Y$. В качестве подпространства $V_{+}$в $\mathrm{H}_{1}\left(\Sigma, \mathbb{Z}_{2}\right)$ нужно рассмотреть сумму образов гомоморфизмов id $-\mathcal{F}_{+}(\gamma)$ для всех $\gamma \in \pi_{1}\left(Y_{+}^{\circ}\right)$ и аналогично определить подпространство $V_{-}$. В остальном доказательство проводится без изменений.

Напротив, основной момент в доказательстве следующей леммы - классификация линейчатых симплектических 4-многообразий.

Лемма 3.4. Ранги $\operatorname{rank} \mathrm{H}_{1}\left(X, \mathbb{Z}_{2}\right)$ u $\operatorname{rank} \mathrm{H}_{1}\left(X^{\prime}, \mathbb{Z}_{2}\right)$ четны.

ДокАЗАТЕЛЬство. Из классификации линейчатых симплектических четырехмерных многообразий (см. [36]) следует, что конечной последовательностью раздутий и стягиваний многообразие $X$ можно перевести в произведение $S^{2} \times Y$, где $Y$ - замкнутая ориентированная поверхность. Поскольку раздутия не меняют $\pi_{1}(X)$, мы получаем первое утверждение леммы.

Второе утверждение получается аналогичным образом, если показать, что многообразие $X^{\prime}$ также является линейчатым (или раздутием линейчатого). Заметим сначала, что отображение склейки $\Psi: X_{\Gamma} \rightarrow X_{\Gamma}$ может быть продолжено до симплектоморфизма некоторой окрестности $X_{\Gamma}$. Следовательно, на многообразии $X^{\prime}$ существует симплектическая форма $\omega^{\prime}$, совпадающая на его подмножествах $X_{Y_{ \pm}}$с исходной формой $\omega$ на многообразии $X$.

Воспользуемся теперь специфической структурой формы $\omega$ и монодромии семейства $\mathrm{pr}: X \rightarrow Y$. Напомним, что многообразие $X$ было симплектическим раздутием исходного многообразия $X_{0}$. Поэтому существуют симплектические сечения $E_{1}, \ldots, E_{N} \subset X$ проекции pr: $X \rightarrow S^{2}$ такие, что для класса когомологий $[D]:=[\Sigma]+\sum_{i}\left[E_{i}\right] \in \mathrm{H}_{2}(X, \mathbb{Z})$ выполнено неравенство $c_{1}(X) \cdot[D]>0$. Сечения $E_{i}$ - это просто исключительные сферы раздутий. Поскольку $E_{i}$ не пересекаются с $K$, они выживают и в $X^{\prime}$, так что мы получаем симплектические сечения $E_{1}^{\prime}, \ldots, E_{N}^{\prime}$ в $X^{\prime}$. Более того, для класса $\left[D^{\prime}\right]:=[\Sigma]+\sum_{i}\left[E_{i}^{\prime}\right] \in \mathrm{H}_{2}(X, \mathbb{Z})$ выполняется аналогичное неравенство $c_{1}\left(X^{\prime}\right) \cdot\left[D^{\prime}\right]>0$. В этой ситуации, из

\footnotetext{
${ }^{3}$ Ср. п. 1.1 в [39]; приводимое ниже доказательство было предложено В. М. Харламовым.
} 
классификации МакДафф и Саламона (см. [36, следствие 1.5]) вытекает, что $X^{\prime}$ действительно является раздутием линейчатого симплектического многообразия. Лемма доказана.

Полученное противоречие показывает, что в условиях основной теоремы монодромия семейства pr: $X \rightarrow Y$ не может иметь вид, описанный в предложении 3.1. Тем самым, основная теорема доказана.

\section{Список литературы}

1. J. Amorós, F. Bogomolov, L. Katzarkov, T. Pantev, "Symplectic Lefschetz fibrations with arbitrary fundamental groups", J. Differential Geom., 54:3 (2000), 489-545.

2. В. И. Арнольд, А. Б. Гивенталь, "Симплектическая геометрия”, Динамические системь - 4, Итоги науки и техн. Сер. Соврем. пробл. мат. Фундам. направления, 4, ВИНИТИ, М., 1985, 5-135; англ. пер.: V. I. Arnol'd, А. B. Givental', "Symplectic geometry", Dynamical systems. IV. Symplectic geometry and its applications, Encyclopaedia Math. Sci., 4, Springer-Verlag, Berlin, 1990, 1-136.

3. В.И. Арнольд, А.Н. Варченко, С.М.Гусейн-Заде, Особенности дифферениируемых отображений. Монодромия и асимптотики интегралов, Наука, М., 1984; англ. пер.: V. I. Arnol'd, S. M. Gusejn-Zade, A. N. Varchenko, Singularities of differentiable maps. Vol. II: Monodromy and asymptotics of integrals, Monogr. Math., 83, Birkhäuser, Boston, MA, 1988.

4. M. Audin, "Quelques remarques sur les surfaces lagrangiennes de Givental", J. Geom. Phys., 7:4 (1990), 583-598.

5. D. Auroux, "A stable classification of Lefschetz fibrations", Geom. Topol., 9 (2005), 203-217.

6. D. Auroux, S. K. Donaldson, L. Katzarkov, "Luttinger surgery along Lagrangian tori and non-isotopy for singular symplectic plane curves", Math. Ann., 326:1 (2003), 185-203.

7. D. Auroux, V. Muñoz, F. Presas, "Lagrangian submanifolds and Lefschetz pencils", J. Symplectic Geom., 3:2 (2005), 171-219.

8. J. S. Birman, "Mapping class groups of surfaces", Proceedings of Conference on Braids (Santa Cruz, CA, 1986), Contemp. Math., 78, Amer. Math. Soc., Providence, RI, 1988, 13-43.

9. J.S. Birman, Braids, links, and mapping class groups, Annals of Math. Studies, 82, Princeton Univ. Press, Princeton, NJ; Univ. of Tokyo Press, Tokyo, 1975.

10. J. S. Birman, B. Wajnryb, "Errata: Presentations of the mapping class group", Israel J. Math., 88:1-3 (1994), 425-427.

11. F. Bogomolov, L. Katzarkov, T. Pantev, "Hyperelliptic Szpiro inequality", J. Differential Geom., 61:1 (2002), 51-80.

12. F. Bogomolov, Yu. Tschinkel, "Simple examples of symplectic four-manifolds with exotic properties", Acta Appl. Math., 75:1-3 (2003), 25-28.

13. E. Brieskorn, K. Saito, "Artin-Gruppen und Coxeter-Gruppen", Invent. Math., 17:4 (1972), 245-271.

14. К. С. Браун, Когомологии групn, Наука, М., 1987; пер. с англ.: K.S. Brown, Cohomology of groups, Grad. Texts in Math., 87, Springer-Verlag, New YorkHeidelberg-Berlin, 1982.

15. Н. Бурбаки, Элементы математики. Группы и алгебры Ли. Гл. 4-6: Группы Кокстера и системы Титса. Группь, порожденные отражениями. Системы корней, Мир, М., 1972; пер. с фр.: N. Bourbaki, Éléments de mathématique. Fasc. XXXIV. Groupes et algèbres de Lie. Chapitre IV: Groupes de Coxeter et systèmes 
de Tits. Chapitre V: Groupes engendrés par des réflexions. Chapitre VI: systèmes de racines, Actualités Scientifiques et Industrielles, 1337, Hermann, Paris, 1968.

16. Д. Коллинз, Х. Цишанг, "Комбинаторная теория групп и фундаментальные группы", Алгебра - 7, Итоги науки и техн. Сер. Соврем. пробл. мат. Фундам. направления, 58, ВИНИТИ, М., 1990, 5-190; англ. пер.: D. J. Collins, H. Zieschang, "Combinatorial group theory and fundamental groups", Algebra VII. Combinatorial group theory. Applications to geometry, Encyclopaedia Math. Sci., 58, Springer-Verlag, Berlin, 1993, 1-166.

17. M. Dehn, "Die Gruppe der Abbildungsklassen", Acta Math., 69:1 (1938), 135-206.

18. P. Deligne, "Les immeubles des groupes de tresses généralisés", Invent. Math., 17:4 (1972), 273-302.

19. S.K. Donaldson, "Lefschetz fibrations in symplectic geometry", Proceedings of the International Congress of Mathematicians (Berlin, 1998), Doc. Math., Extra Vol. II, 1998, 309-314.

20. Ya. Eliashberg, L. Polterovich, "New applications of Luttinger's surgery", Comment. Math. Helv., 69:1 (1994), 512-522.

21. D. Gatien, F. Lalonde, "Holomorphic cylinders with Lagrangian boundaries and Hamiltonian dynamics", Duke Math. J., 102:3 (2000), 485-511.

22. S. Gervais, "Presentation and central extensions of mapping class groups", Trans. Amer. Math. Soc., 348:8 (1996), 3097-3132.

23. А. Б. Гивенталь, “Лагранжевы вложения поверхностей и раскрытый зонтик Уитни”, Функи. анализ и его прил., 20:3 (1986), 35-41; англ. пер.: А. В. Givental', "Lagrangian imbeddings of surfaces and unfolded Whitney umbrella", Funct. Anal. Appl., 20:3 (1986), 197-203.

24. R. E. Gompf, A. I. Stipsicz, 4-manifolds and Kirby calculus, Grad. Stud. Math., 20, Amer. Math. Soc., Providence, RI, 1999.

25. R. E. Gompf, "Toward a topological characterization of symplectic manifolds", J. Symplectic Geom., 2:2 (2004), 177-206.

26. Ф. Гриффитс, Дж. Харрис, Принципы алгебраической геометрии, Мир, М., 1982; пер. с англ.: Ph. Griffiths, J. Harris, Principles of algebraic geometry, Wiley, New York, 1978.

27. J. E. Humphreys, Reflection groups and Coxeter groups, Cambridge Stud. Adv. Math., 29, Cambridge Univ. Press, Cambridge, 1990.

28. N. V. Ivanov, "Mapping class groups", Handbook of geometric topology, Elsevier, Amsterdam, 2002, 523-633.

29. D. Johnson, "The structure of the Torelli group. I. A finite set of generators for $\mathscr{I} "$, Ann. of Math. (2), 118:3 (1983), 423-442.

30. D. Johnson, "A survey of the Torelli group", Low-dimensional topology (San Francisco, CA, 1981), Contemp. Math., 20, Amer. Math. Soc., Providence, RI, 1983, 165-179.

31. V. Kanev, Irreducibility of Hurwitz spaces, Preprint № 241, Dipartimento di Matematica, Universitá di Palermo; arXiv: math. AG/0509154.

32. В. М. Харламов, Вик. С. Куликов, "О брэйд-монодромных разложениях на множители”, Изв. РАН. Сер. матем., 67:3 (2003), 79-118; англ. пер.: V. M. Kharlamov, V. S. Kulikov, "On braid monodromy factorizations", Izv. Math., 67:3 (2003), 499-534.

33. K. M. Luttinger, "Lagrangian tori in $\mathbb{R}^{4}$ ", J. Differential Geom., 42:2 (1995), 220-228.

34. Р. Линдон, П. Шупп, Комбинаторная теория групn, Мир, М., 1980; пер. с англ.: R. C. Lyndon, P. E. Schupp, Combinatorial group theory, Ergebnisse der Mathematik und ihrer Grenzgebiete, 89, Springer-Verlag, Berlin-New-York, 1977.

35. M. Matsumoto, "A presentation of mapping class groups in terms of Artin groups and geometric monodromy of singularities", Math. Ann., 316:3 (2000), 401-418.

36. D. McDuff, D. Salamon, "A survey of symplectic 4-manifolds with $b_{+}=1$ ", Turkish J. Math., 20:1 (1996), 47-60. 
37. K. Mohnke, How to (symplectically) thread the eye of a (Lagrangian) needle, arXiv: math.SG/0106139.

38. С. Ю. Немировский, "Пучки Лефшеца, функции Морса и лагранжевы вложения бутылки Клейна”, Изв. РАН. Сер. матем., 66:1 (2002), 153-166; англ. пер.: $\mathrm{S}$. Yu. Nemirovski, "Lefschetz pencils, Morse functions, and Lagrangian embeddings of the Klein bottle", Izv. Math., 66:1 (2002), 151-164.

39. С. Ю. Немировский, "Гомологический класс лагранжевой бутылки Клейна", Изв. РАН. Сер. матем., 73:4 (2009), 37-48; англ. пер.: S. Yu. Nemirovski, "Homology class of a Lagrangian Klein bottle", Izv. Math., 73:4 (2009), 689-698.

40. J. Powell, "Two theorems on the mapping class group of a surface", Proc. Amer. Math. Soc., 68:3 (1978), 347-350.

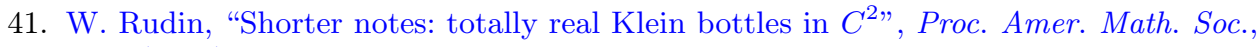
82:4 (1981), 653-654.

42. B. Wajnryb, "A simple presentation for the mapping class group of an orientable surface", Israel J. Math., 45:2-3 (1983), 157-174.

B. В. Шевчишин (V. V. Shevchishin)

University of Bonn, Mathematical Institute, Germany

E-mail: sewa@math.uni-bonn.de
Поступило в редакцию 26.03.2007 